\title{
Long-Term Modelling of Composite Pavement Performance
}

by

Evangelia Manola

Thesis submitted in partial fulfilment of the requirements for the award of the degree of Doctor of Philosophy

De Montfort University

January 2019 


\section{Abstract}

The rehabilitation of rigid (concrete) pavements with the placement of an asphalt overlay is a common maintenance technique, which results in a structure known as a composite pavement. The most common form of distress in this type of pavement is reflection cracking which can be due to traffic and/or climatic loading. This thesis is focused on the prediction of reflective cracking as a composite pavement response due to traffic and thermal loading. The model is the first of its kind to include factors such as dynamic vehicle loading in modelling the long-term performance of flexible composite pavement structures.

The mechanisms of traffic driven reflective cracking are investigated, as well as a thermal driven cracking model. The above models are combined with a dynamic vehicle model into a whole-life model and its framework is presented. A parametric analysis investigates the sensitivity of the predictions to the variation of parameters: asphalt elastic modulus and thickness, concrete elastic modulus and thickness, subbase elastic modulus and thickness and subgrade elastic modulus.

Finally, the main validation analysis is presented, with application of the whole model for representative pavement sections from 6 states in the USA. The input data is found in the Long-Term Pavement Performance Infopave database, which contains performance and traffic data from monitored in-service road sections in North America. A comparison is made between the outcome predictions and actual field measurements of transverse cracks on the relevant pavement sections. The selected pavement sections belong to two climatic regions: wet/freeze zone, wet/non-freeze.

Predictions for certain states show matching results approximately to the actual development of reflective cracking at different periods after the placement of the asphalt overlay. It was found that the asphalt material properties have a big influence on the final outcome making critical its estimation. Temperature variations have also been taken in account and it has been seen that they can influence the result showing that thermal reflective cracking can be dominant. It was also discovered that knowledge of the state (crack spacing, severity of cracks) is highly important to the identification and prediction of reflective cracks. It was important to also investigate the process of both directions of cracking, bottom to up and top to down, identifying their dominance in each case.

In the final chapter conclusions and recommendations for future work are presented. 


\section{Acknowledgements}

The work in this thesis would not have been possible without the help and support of several individuals and organisations whose contributions I would like to acknowledge here:

Firstly, I would like to thank my research supervisor and mentor Prof. Andy Collop, to whom I am grateful for the suggestion of the project and the continuous support throughout my PhD study. His invaluable advice along with his immense knowledge, motivation and encouragement were key in the completion of this research project. I would also like to thank my second supervisor Dr. Yong Sun and the research team in DIGITS department of De Montfort University, where I was based and also cooperated with many of its researchers on a daily basis. Specifically, I would like to thank Prof. David Elizondo, Dr. Daniel Paluszczyszyn and Dr. Ben Passow who were always there for good advice and guidance.

I would also like to express my sincere gratitude to Dr. Nick Thom, from University of Nottingham for our cooperation on this research study. His guidance and his permission to access past unpublished research material as well as our extensive discussions, have been a privilege and major help for the fruition of this project.

Last but not least, this $\mathrm{PhD}$ journey would not have been the same or even maybe possible without the invaluable help of my parents Stella and Vangelis, who gave me the courage and the will to continue every step of the way with their endless in me confidence and love, as well as my brothers Manos and Nikos, and my sister-in-law and friend Ersi. Their ongoing and never-ending support both practical and emotional are some of the reasons I am able to write this today. I have also been lucky to have been supported continuously from my Leicester $\mathrm{PhD}$ buddies Louise, Cassie, Christian and Claire with whom I shared the joys and difficulties of the PhD journey. Special thank you also to friends I made in Leicester, especially Betty as well as friends from home Nafsika and Maria for all of their encouragement throughout this time. 


\section{Declaration}

The work presented in this thesis was conducted at De Montfort University, Leicester between July 2014 and December 2018. I declare that the work is my own, except where specific reference has been made to the work of others and has not been submitted for a degree of another University. 


\section{Table of Contents}

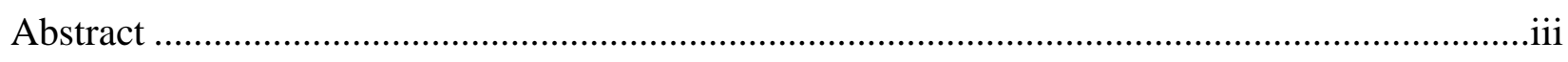

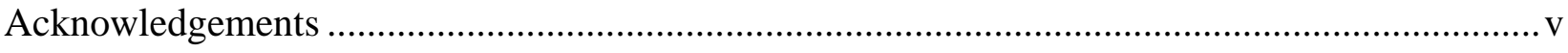

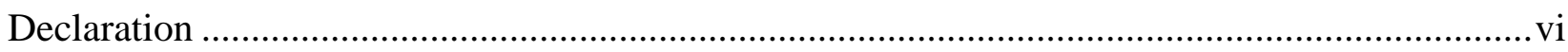

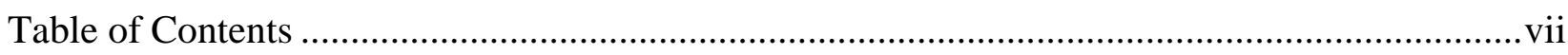

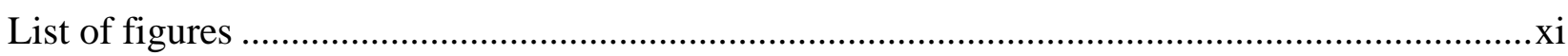

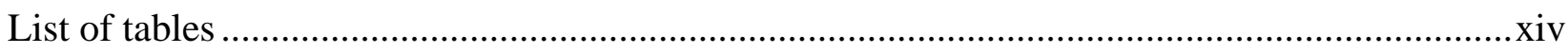

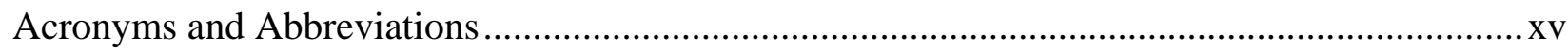

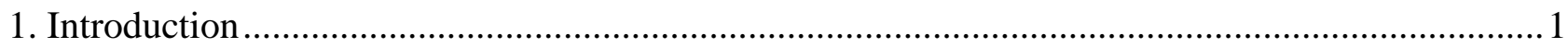

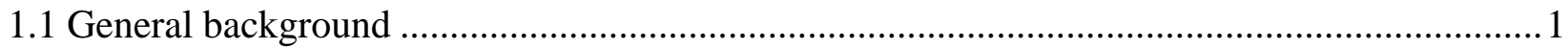

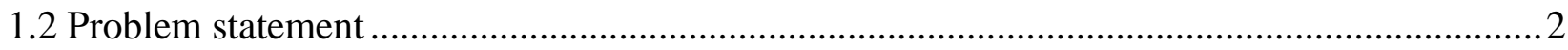

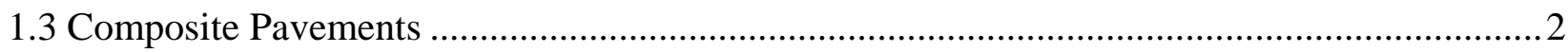

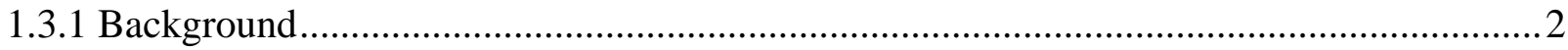

1.3.2 Reflection cracking .............................................................................................

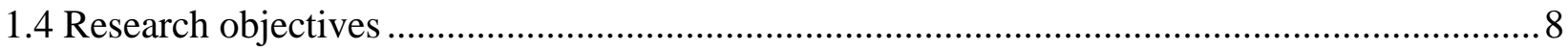

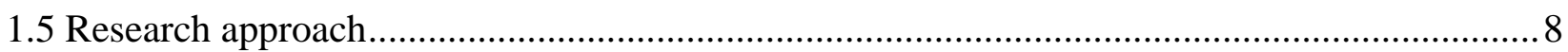

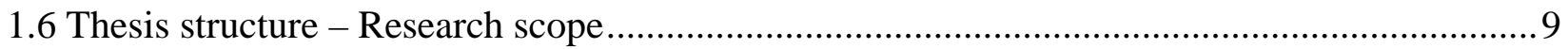

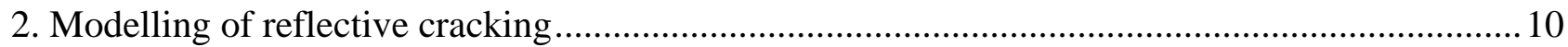

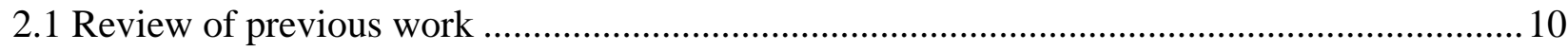

2.1.1 Simple mechanistic models ........................................................................................10

2.1.2 Finite element analysis (with or without fracture mechanics)............................................12

2.2 Olcrack - Theoretical modelling ......................................................................................... 16

2.2.1 Bottom-up cracking mechanism .............................................................................18

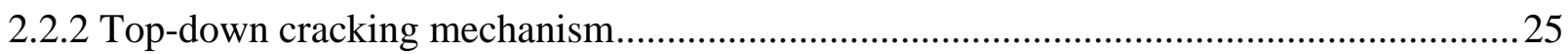

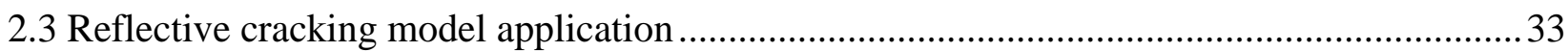

2.3.1 Example simulation of typical rehabilitated rigid pavement ...............................................33

2.3.2 Example simulation of typical new flexible composite pavement section...........................35

2.4 Parametric study of traffic driven reflective cracking model...................................................35

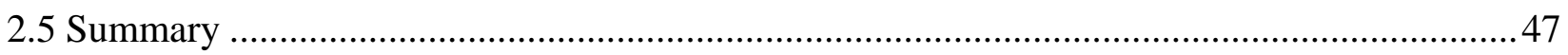




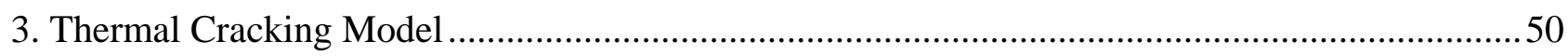

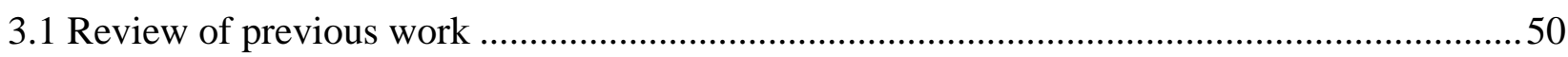

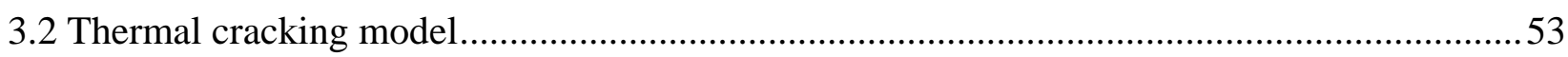

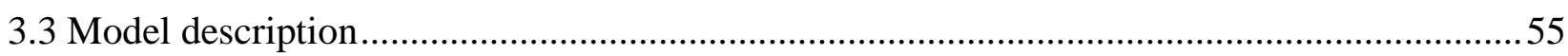

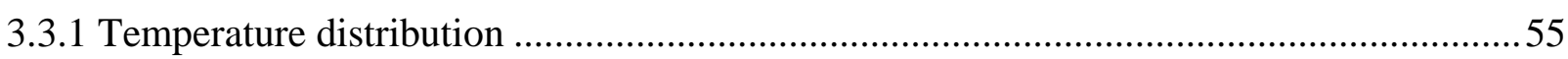

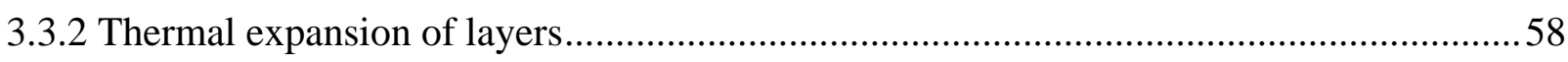

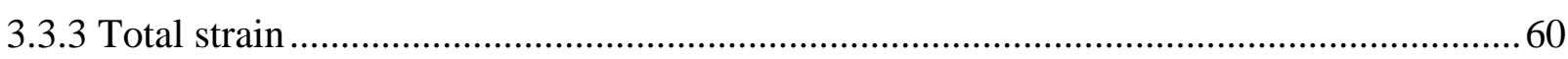

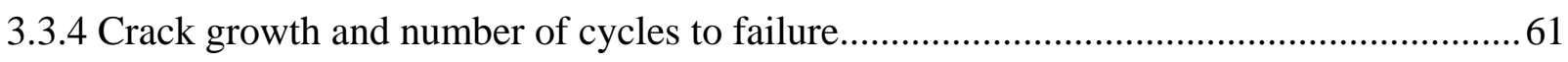

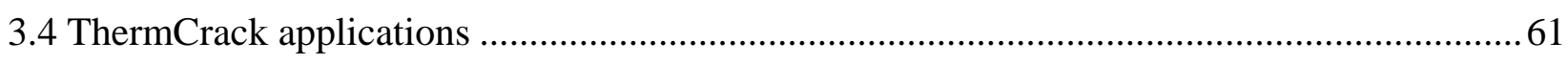

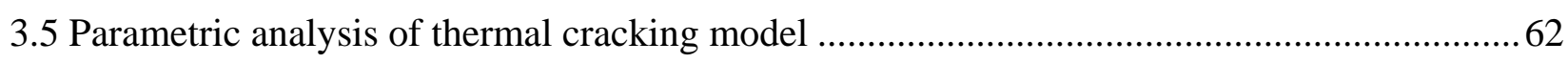

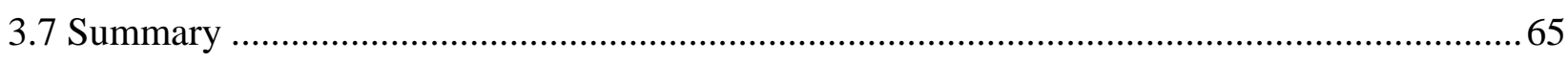

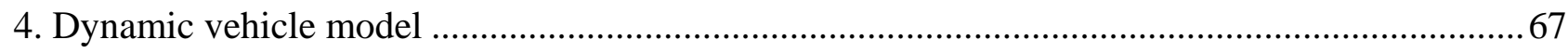

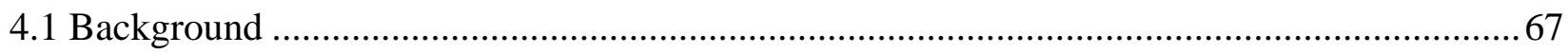

4.1.1 Vehicle loading \& Pavement performance.....................................................................67

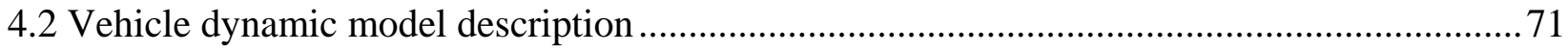

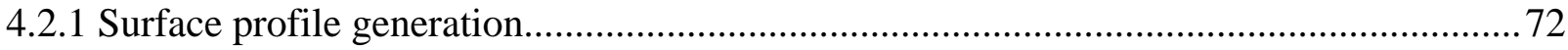

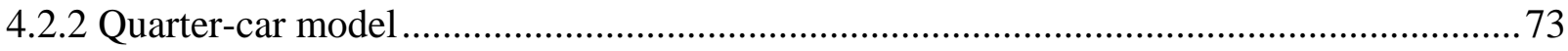

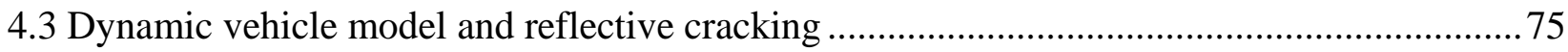

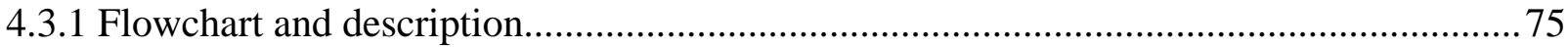

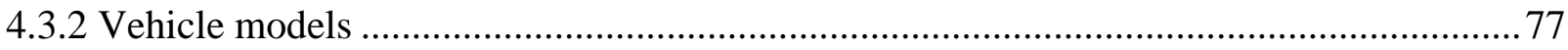

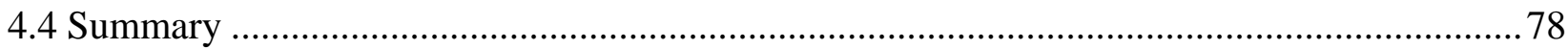

5. Whole life long-term flexible composite pavement model...................................................... 79

5.1 Whole Life Pavement Performance Models - Previous work ............................................... 79

5.2 Whole life long-term flexible composite model description...............................................80

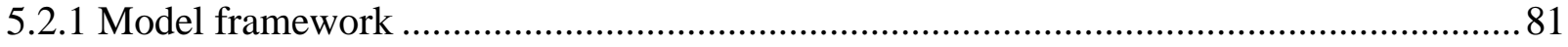

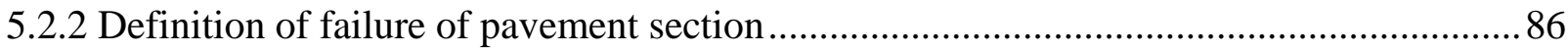

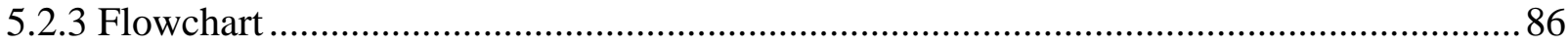

5.3 Application of model for pavement with baseline values ..................................................... 89

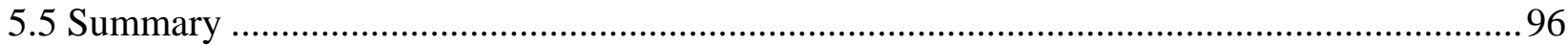

6. Parametric Study of whole-life flexible composite pavement model ........................................98

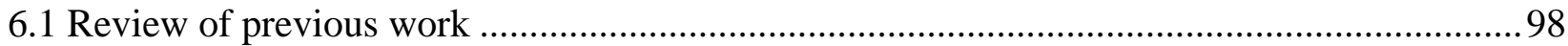




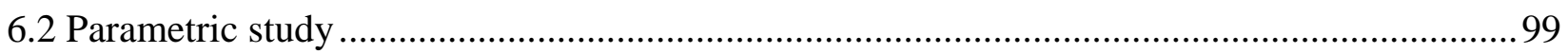

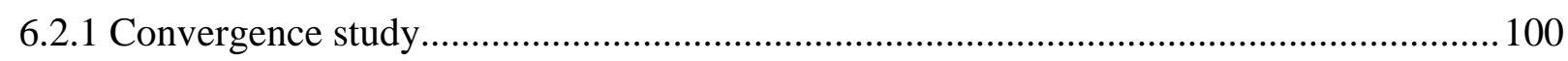

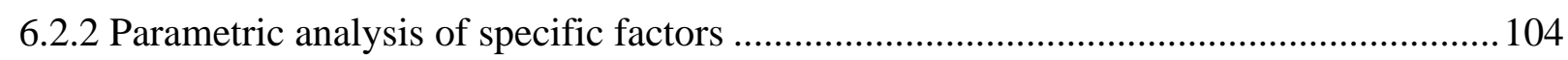

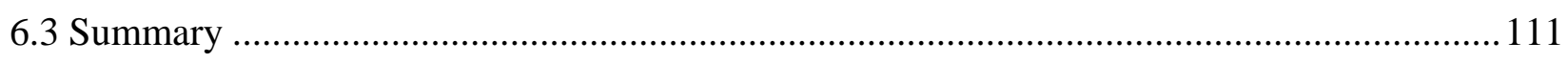

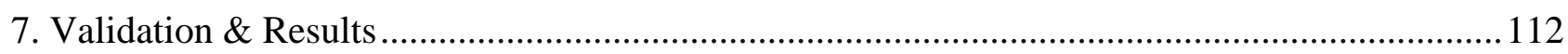

7.1 Long-Term Pavement Performance database.................................................................. 112

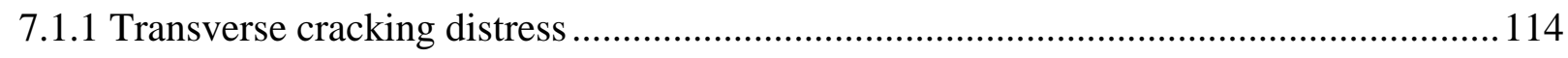

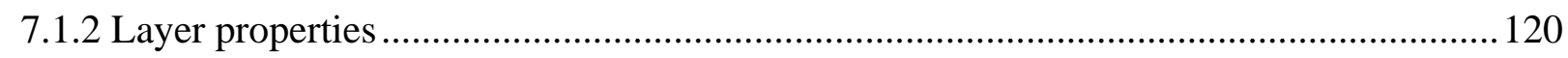

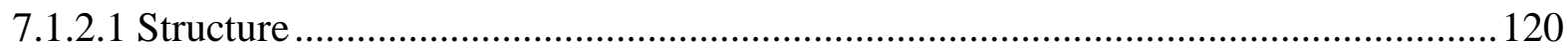

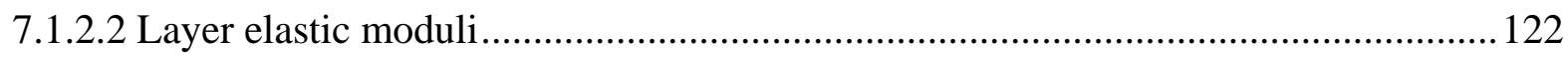

7.1.3 Asphalt modulus temperature correction................................................................. 125

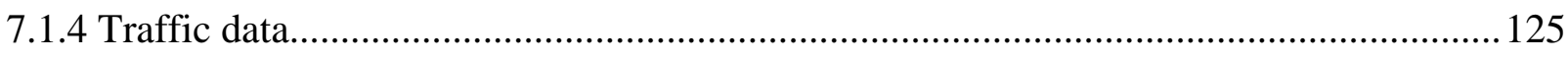

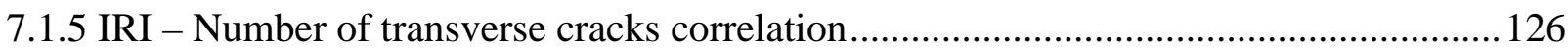

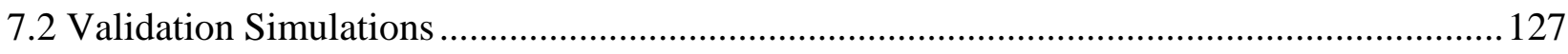

7.2.1 Examples of failure with reflective cracking at a single location ..................................127

7.2.2 Whole-life flexible composite model predictions........................................................ 132

7.3 Thermal cracking model example simulations................................................................... 139

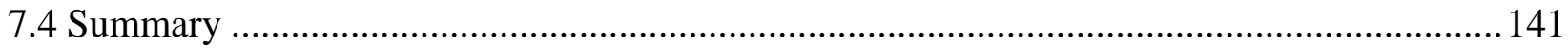

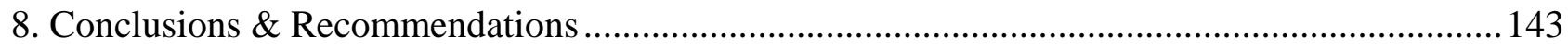

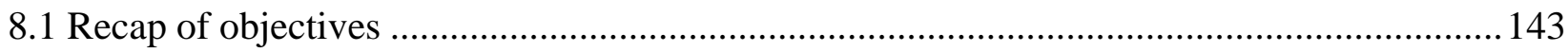

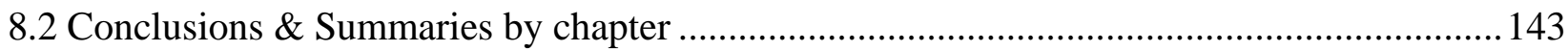

8.2.1 Chapter 2 - Modelling of reflective cracking ............................................................. 143

8.2.2 Chapter 3 - Modelling of thermal reflective cracking .................................................. 145

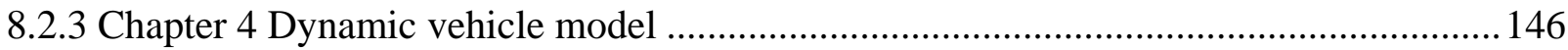

8.2.4 Chapter 5 - Whole life long-term flexible composite pavement model ..........................147

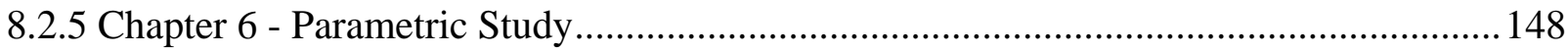

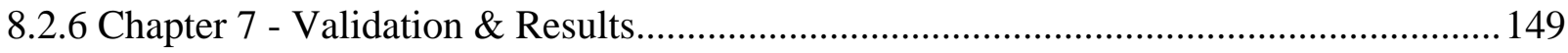

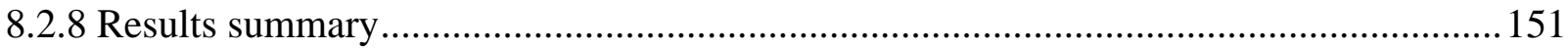

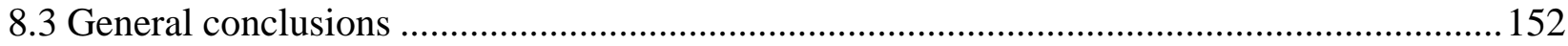

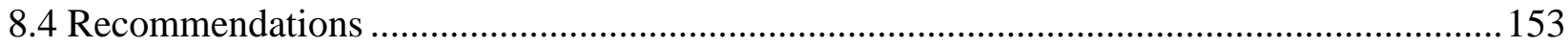

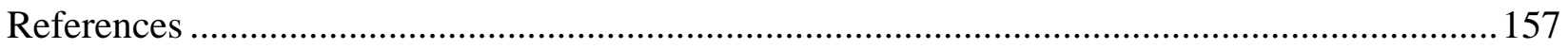


Appendix A - Equations 165

Appendix B - Matlab code... 171 


\section{List of figures}

Figure 1: Stresses in the asphalt overlay due to a wheel load. (Kohale and Lytton, 2000) ...............7

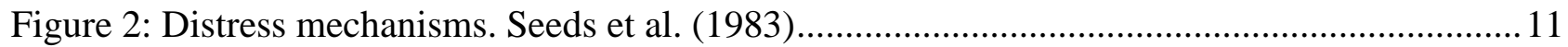

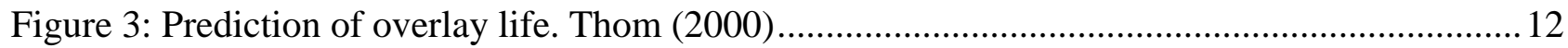

Figure 4: Number of days of crack growth. (Lytton, 2010).......................................................13

Figure 5: General structure of composite pavements.................................................................. 17

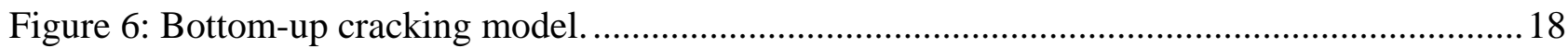

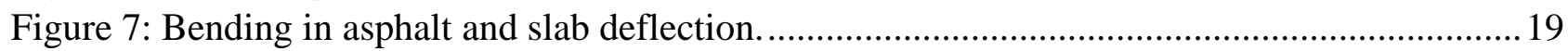

Figure 8: Flow chart for bottom-up cracking mechanism.............................................................20

Figure 9: Stress distribution with bottom crack in asphalt......................................................22

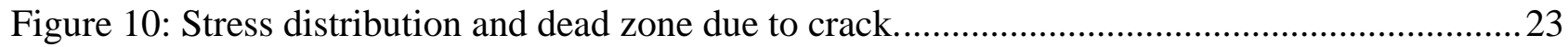

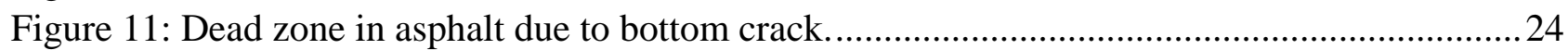

Figure 12: Top-down cracking mechanism. ................................................................................... 25

Figure 13: Top-down cracking mechanism due to wheel load. ....................................................26

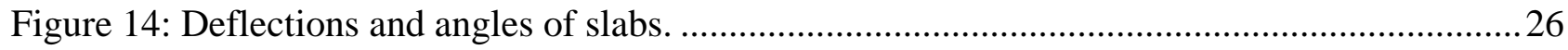

Figure 15: Flow chart for top-down cracking mechanism.........................................................28

Figure 16: Stress distribution with top and bottom crack. ......................................................29

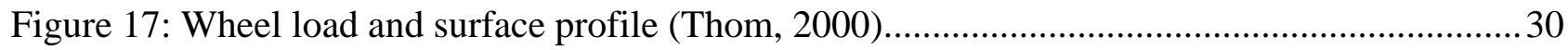

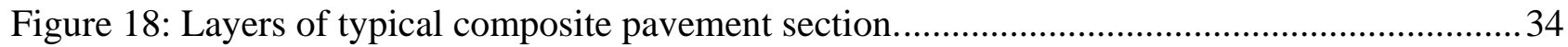

Figure 19: Crack development plot in typical rehabilitated rigid pavement section.......................34

Figure 20: Crack development plot in typical new flexible composite section..............................35

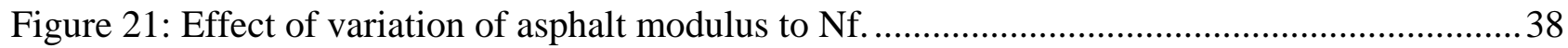

Figure 22: Effect of variation of asphalt layer thickness to $\mathrm{Nf}$.................................................40

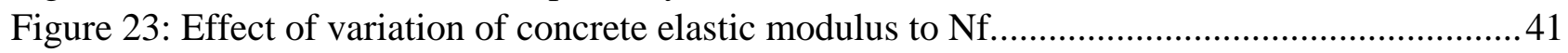

Figure 24: Effect of variation of concrete layer thickness to Nf............................................... 42

Figure 25: Effect of variation of subbase elastic modulus to Nf. ................................................ 43

Figure 26: Effect of variation of subbase layer thickness to Nf....................................................4 44

Figure 27: Effect of variation of subgrade elastic modulus to Nf.................................................44

Figure 28: Effect of variation of crack spacing to Nf. .................................................................. 45

Figure 29: Effect of variation of crack shear modulus to Nf. ........................................................46

Figure 30: Parameter variation and result on Nf................................................................48

Figure 31: Thermal stresses in composite pavements. (Thom, 2014) ...........................................52

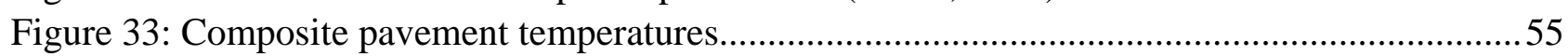

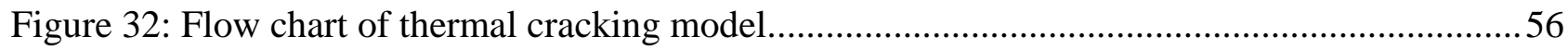

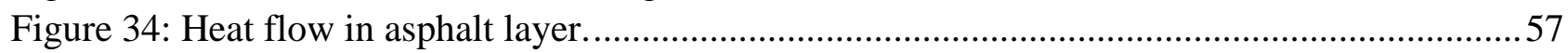

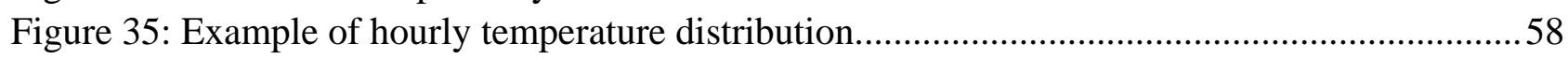

Figure 36: Movement rates between asphalt and CBM layer..................................................59

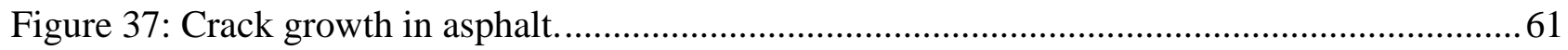

Figure 38: Normalized thermal strain for different parameters. ...............................................63

Figure 39: Normalized no. of thermal cycles to failure for different parameters (a)......................64 
Figure 40: Normalized no. of thermal cycles to failure for different parameters (b).

Figure 41:Various values of the exponent $\mathrm{n}$ in the power law equation and their impact. (Cebon, 1999)

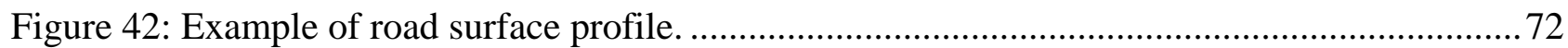

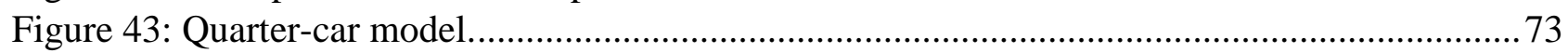

Figure 44: Example of dynamic tyre forces of three different quarter-car models......................... 74

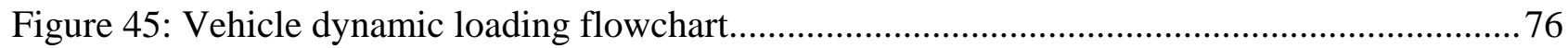

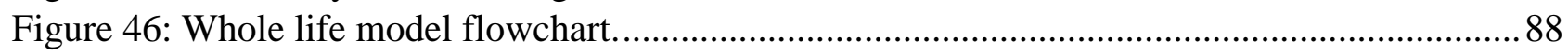

Figure 47: Weekly averaged pavement temperature over 1 year...................................................90

Figure 48: $\log (\mathrm{Mr})-$ Temperature for estimation of slope. ......................................................... 91

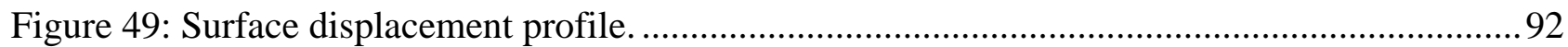

Figure 50: Set of forces representing 3 different vehicle models. .................................................92

Figure 51: Crack development at 1st location (3 m. from start) after 1 month. ...............................94

Figure 52: Crack development at location 39 (117 m. from start) after 4 months...........................94

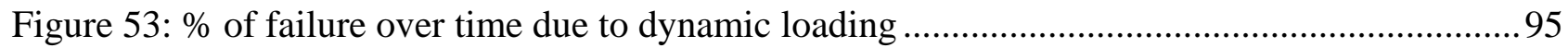

Figure 54: \% of failure over time due to dynamic and static loading...........................................96

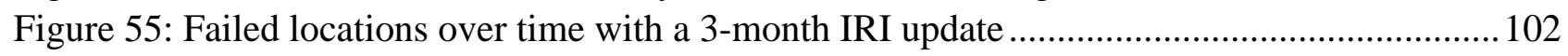

Figure 56: Failed locations over time with a 1-month IRI update ............................................103

Figure 57:Reflective cracking development through asphalt thickness.......................................103

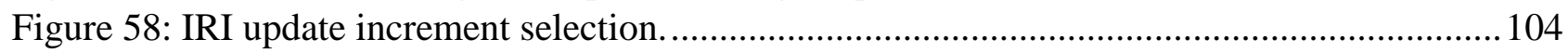

Figure 59: Asphalt elastic modulus variation - \% of failed locations over time............................. 105

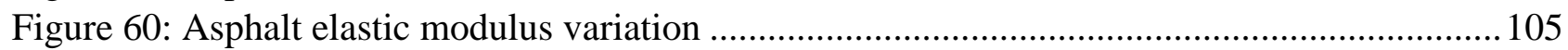

Figure 61: Asphalt thickness variation - \% of failed locations over time......................................106

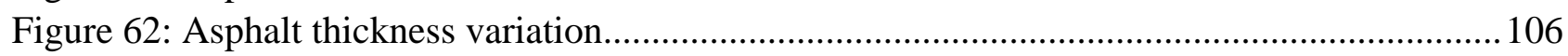

Figure 63: Concrete elastic modulus variation - \% of failed locations over time..........................107

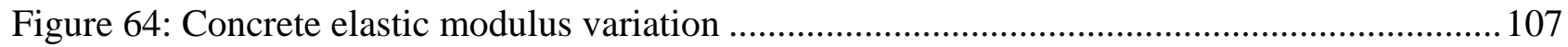

Figure 65: Concrete thickness variation - \% of failed locations over time..................................... 108

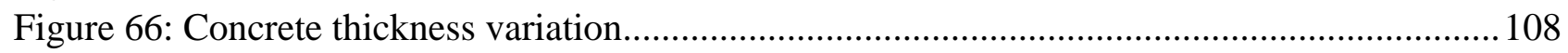

Figure 67: Subbase elastic modulus variation - \% of failed locations over time........................... 109

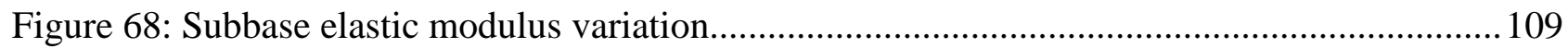

Figure 69: Subbase thickness variation - \% of failed locations over time.....................................109

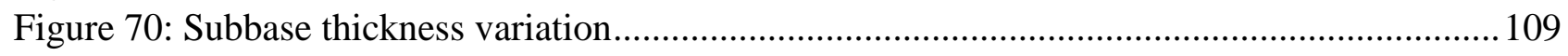

Figure 71: Subgrade elastic modulus variation - \% of failed locations over time .......................... 110

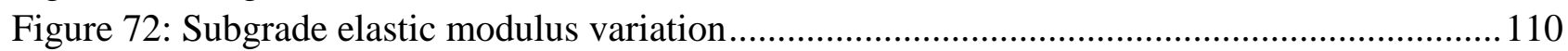

Figures 73(a-f): Transverse cracks per metre along time for 6 states....................................... 115

Figure 74: Ratio of transverse cracks before and after overlay. ................................................ 117

Figure 75: Distress maps showing reflective cracking for state of California..............................118

Figure 76: Distress maps showing reflective cracking for state of Illinois................................... 120

Figure 77: Asphalt elastic modulus on the year after the asphalt overlay for California. ................123

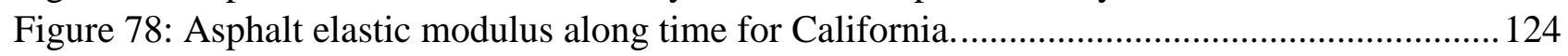

Figure 79(a-f): Correlation between IRI - No. of transverse cracks per m. .................................127 
Figure 80(a-f): Idealised composite pavements from each state

Figure 81(a-f): Bottom-up and top-down level of reflective cracking at $1^{\text {st }}$ and $5^{\text {th }}$ location.

Figure 82: \% of failed locations over time due to dynamic and static traffic loading - Alabama. ..134

Figure 83: \% of failed locations over time due to dynamic and static traffic loading - California. 135

Figure 84: \% of failed locations over time due to dynamic and static traffic loading - Illinois. .....136

Figure 85: \% of failed locations over time due to dynamic and static traffic loading - Iowa..........137

Figure 86: \% of failed locations over time due to dynamic and static traffic loading - Pennsylvania.

Figure 87: \% of failed locations over time due to dynamic and static traffic loading - Tennessee. 138

Figure 88: Damage failure due to thermal cracking. 140 


\section{List of tables}

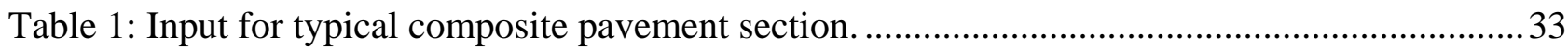

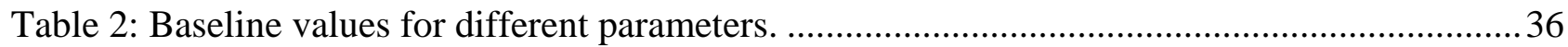

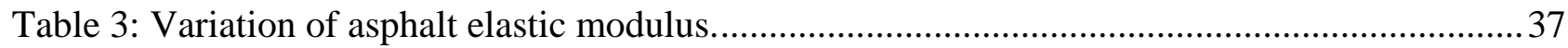

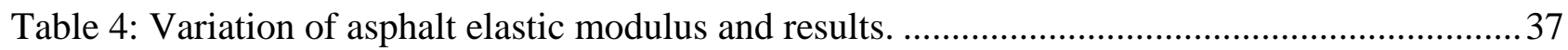

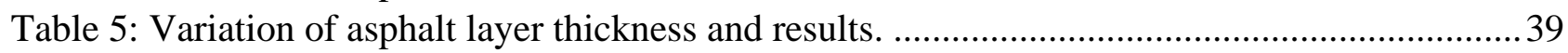

Table 6: Variation of concrete elastic modulus and results. ........................................................41

Table 7: Variation of concrete layer thickness and results. ........................................................42

Table 8: Variation of subbase elastic modulus and results. ..........................................................42

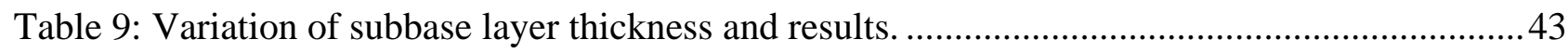

Table 10: Variation of subgrade elastic modulus and results. ...................................................44

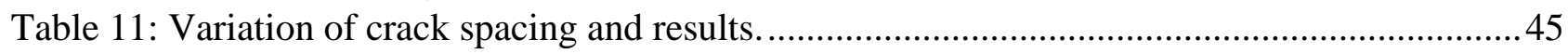

Table 12: Variation of crack shear modulus and results.................................................................46

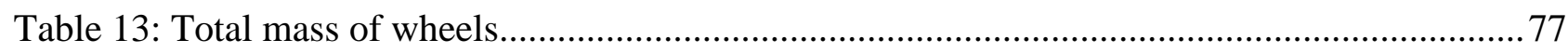

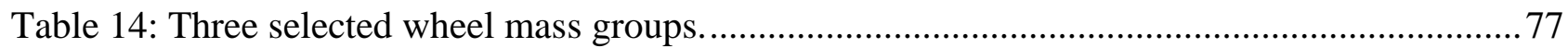

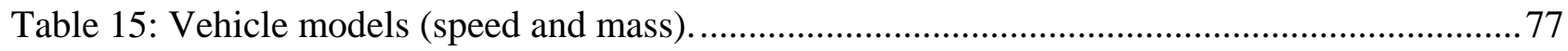

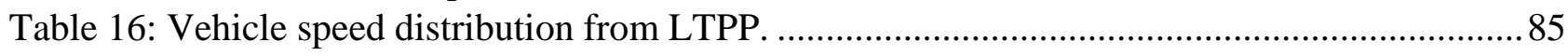

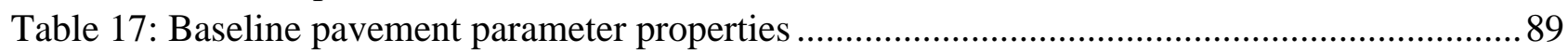

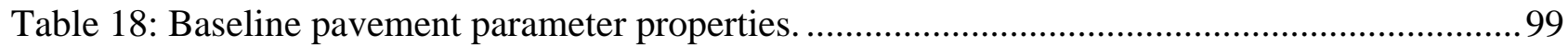

Table 19: Time frequency for parameter update.................................................................... 101

Table 20: Time in months required for specific \% of failure with asphalt modulus variation ........ 105

Table 21: Time in months required for specific \% of failure with asphalt thickness variation ....... 106

Table 22: Time in months required for specific \% of failure with concrete modulus variation......107

Table 23: Time in months required for specific \% of failure with concrete thickness variation..... 108

Table 24: Time in months required for specific \% of failure with subbase modulus variation.......108

Table 25: Time in months required for specific \% of failure with subbase thickness variation.......109

Table 26: Time in months required for specific \% of failure with subgrade modulus variation ..... 110

Table 27: Basic structural input data from 6 states..................................................................... 121

Table 28: Average elastic moduli data for each state. ................................................................ 124

Table 29: Slopes for elastic modulus temperature correction procedure.....................................125

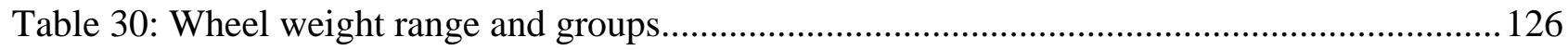




\begin{tabular}{|c|c|}
\hline${ }^{\circ} \mathrm{C}$ & degree Celsius \\
\hline CBM & Cement Bound Material \\
\hline CRCP & Continuously Reinforced Concrete Pavements \\
\hline DMRB & Design Manual for Roads and Bridges \\
\hline $\mathrm{E}$ & Elastic Modulus \\
\hline ESAL & Equivalent Single Axle Load \\
\hline HBM & Hydraulic Bound Base \\
\hline IRI & International Roughness Index \\
\hline $\mathrm{J} / \mathrm{kg} / \mathrm{K}$ & Joules per kilogram per Kelvin \\
\hline JPCP & Jointed Plain Concrete Pavements \\
\hline K & Kelvin \\
\hline $\mathrm{kg}$ & kilogram \\
\hline $\mathrm{km} / \mathrm{h}$ & kilometers per hour \\
\hline $\mathrm{kN}$ & Kilo-Newton \\
\hline $\begin{array}{l}\text { LTPP } \\
\mathrm{m}\end{array}$ & $\begin{array}{l}\text { Long-Term Pavement Performance } \\
\text { metre }\end{array}$ \\
\hline $\begin{array}{l}\text { MEPDG } \\
\mathrm{mm}\end{array}$ & $\begin{array}{l}\text { Mechanistic-empirical Pavement Design Guide } \\
\text { millimetres }\end{array}$ \\
\hline MN & Mega-Newton \\
\hline $\mathrm{MPa}$ & Megapascal \\
\hline $\mathrm{mph}$ & miles per hour \\
\hline msa & $\begin{array}{l}\text { million standard axles } \\
\text { National Cooperative Highway Research }\end{array}$ \\
\hline NCHRP & Program \\
\hline PLLP & Perpetual Long-Life Pavements \\
\hline S & second \\
\hline SHRP & Strategic Highway Research Program \\
\hline SMA & Stone Matrix Asphalt \\
\hline TRL & Transport Research Laboratory \\
\hline $\mathrm{V}$ & Poisson's ratio \\
\hline $\mathrm{W} / \mathrm{mK}$ & Watts per meter-Kelvin \\
\hline
\end{tabular}




\section{Introduction}

\subsection{General background}

The need for communication and travelling has led to the development of a massive road and infrastructure system more or less in all the continents. The transportation of people and goods in an efficient and economical way, with safety as a priority on highway networks, comes with great challenges that each country approaches according to their needs.

These different needs regard the local climate, the available natural resources in the area as well as economic reasons. In order to meet these needs, research is ongoing and developing regarding different types of construction of roads and road materials. Research is extensive when it comes to new materials or improving existent ones, as well as new design methods and technologies that will aid this sector in the construction of cost-efficient and efficient road networks. The highway authorities that are responsible, aim for developing long lasting structures while simultaneously using minimum disruptive procedures.

The road system that was constructed 40 or 50 years ago however has already deteriorated and efforts have been made for effective replacement or maintenance of pre-existing structures. The methods that are used are new and need to adapt to extreme events that might happen due to climate change. These could be very dry and hot weather, as well as water due to flooding. Environmental conditions as well as irregular heavy loading plays an important role to the rate of deterioration of each pavement.

The topic of this thesis is focused on the typical distresses of a composite pavement. Composite pavements are multi-layer structures which combine cementitious and asphaltic materials. In this case it is an asphalt layer on top of a cement bound base layer or a lean concrete layer. The typical distress that develops in this type of structure is reflective cracking and an effort to model these pavements and predict this distress is presented in this research study.

The benefits of composite pavements have been shown to be long service life, very good characteristics of surface texture, structural capacity and a quick replacement procedure when needed (Rao, 2013). Statistics in both Europe and the United States show that approximately 30\% of the urban interstate system and around $20 \%$ of the rural interstate system is categorized as a composite 
pavement. Composite systems remain a sustainable and economic solution because of the possibility of use of lower quality material in the top and lower layers that could easily include percentages of recycled material.

\subsection{Problem statement}

Composite pavements are a solution to the problem of rigid pavements in need of rehabilitation, contributing to sustainability by reusing the existing pavement layers as structural members of the new pavement. However, despite its benefits it also presents certain distresses that may influence the pavement's performance and its life. Specifically, they develop the unique distress of reflective cracking which increases the roughness of the pavement, as well as to contributing to a weak load transfer in the underlying layers. Cracks that have reflected to the surface of the asphalt layer, if they remain untreated, can allow water ingress leading to the damage of the lower pavement layers.

The problem of reflective cracking is also a complex one that needs addressing. That entails discovering the parameters that drive its rate of development as well as the specific locations where it appears. Several researchers have tried to address this matter in different ways, but mainly taking into account a static load and not vehicle dynamic loading.

\subsection{Composite Pavements}

\subsubsection{Background}

Pavements can be considered as multi-layered structures consisting of materials in horizontal layers with a durable surfacing. The primary function of the layers is to distribute the loads to the underlying soils. To achieve this, the upper pavement typically comprises higher quality materials and is able to spread the applied loads and protect the underlying layers from excessive stress and failure. Pavements generally belong to two broad categories: flexible and rigid (Yoder and Witczak, 1975). However, combination of the characteristics of both types in the design of a pavement, has resulted in a type of pavement known as a composite pavement.

Flexible pavements are typically constructed with asphalt and granular materials whereas rigid pavements are typically constructed using concrete (Huang, 1993). The main difference between the flexible and rigid type of pavement is the way the load spreads underneath the structural layer and into the subgrade (Yoder and Witczak, 1975). The concrete layer in the rigid pavements spreads the 
load to a wider area of the subgrade, whereas the bituminous layer in the flexible pavement transfers the load to a smaller area of the underlying pavement.

Composite pavements typically comprise an asphaltic surfacing constructed over a hydraulically bound mixture material and can either refer to a new "as designed" pavement structure or a rehabilitated concrete pavement where an asphalt overlay has been added to extend the life of the structure. Composite pavements have been constructed in the USA since the 1950s on a national or local basis, in the form of a concrete base overlaid with a Hot-Mixed Asphalt wearing surface layer. The base would be either a Continuous Reinforced Concrete (CRC) layer or a Jointed Plain Concrete layer. There have also been many constructions in Europe, for example in the Netherlands, Italy and the United Kingdom which aimed to provide low-noise pavements or long-life pavements, and to achieve that, they constructed composite pavements (Rao, 2013).

The Strategic Highway Research Program (Rao, 2013) clearly stated the definition of composite pavements as: “a structure which consists of multiple, structurally significant, layers of different, sometimes heterogeneous composition. Two layers or more must employ dissimilar, manufactured binding agents”. According to the Design Manual for Roads and Bridges (DMRB) in the UK, there are two types of composite pavements; flexible composite and rigid composite. A flexible composite pavement comprises a surfacing of bituminous material over a hydraulically bound mixture (lean concrete) which is the road base. A rigid composite pavement which will not be examined here consists of a bituminous surfacing overlying a Continuously Reinforced Concrete Road base (CRCR).

In the UK, there was an effort to design and develop long life flexible pavements. The design for this type of pavements followed the design of a typical flexible composite pavement by including a lean concrete layer overlaid with an upper asphalt layer. Research reports by the Transport Research Laboratory (TRL) were produced from 1984 (Powell et al., 1984) and a review of the existing flexible composite pavements in the UK regarding their construction and maintenance was undertaken by Parry et al. (1999). $649 \mathrm{~km}$ of flexible composite pavements that were constructed between 1959 and 1987 were reviewed and it was concluded that the current design is sufficient for carrying traffic of at least 100 million standard axles (msa). It was found that pavements with an asphalt layer of 
thickness $200 \mathrm{~mm}$ over a $250 \mathrm{~mm}$ thick cement-bound base are able to carry more than 100 million standard $80 \mathrm{kN}$ axles.

The current design in the Design Manual of Roads and Bridges (DMRB) (Volume 7, Section 2, Part 3 HD 26/06) states that the minimum allowable Hydraulic Bound Base Material (HBM) thickness is 150mm for flexible construction with HBM base. The asphalt thickness is determined from the following equation depending on the design traffic in million standard axles (msa):

$$
H=-16.05 \times(\log N)^{2}+101 \times(\log N)+45.8
$$

Where:

$\mathrm{N}$ : design traffic in msa, up to 400 msa.

In Australia, an approach was developed by Parmeggiani (2012) for the design of long life pavements referred to as PLLP which consisted of an upper asphalt layer over a layer of lean concrete and a cemented subbase. Following the design approach of the UK and the USA the main critical pavement layer responses were considered to be the horizontal tensile strain at the bottom of the asphalt layer, and the horizontal tensile strain at the bottom of the cemented layer and the vertical compressive strain at the top of the subgrade.

One of the most concise efforts to report on the performance and construction techniques of composite pavements was during the SHRP 2 Renewal Project R21 (Tompkins et al., 2010). Pavement sections from the Netherlands, Germany and Austria were reviewed and it was found that they performed well under heavy traffic loading during their 10 to 20-year life. From this report, parameters regarding material properties and performance of the pavements were identified and mechanistic-empirical performance models were validated. Examples from the Netherlands show the placement of a porous asphalt concrete layer over CRCP pavement that was recently placed. The results reported from this showed low noise levels and no reflective cracking from the continuously reinforced concrete pavement (CRCP). In Germany Stone Matrix Asphalt (SMA) was placed either on Jointed Plain Concrete Pavements (JPCP) or CRCP.

Composite pavements typically develop distresses that appear in both flexible and rigid pavements. These distresses can be categorized in three broad groups: fracture, distortion and disintegration. 
Many efforts have been made by researchers to model the major damage mechanisms that develop in pavements. These damage mechanisms belong mainly to the fracture distresses like fatigue cracking, reflective cracking and thermal cracking and to distortion like rutting (Von Quintus, 1979). Other problems that may appear include construction defects, inadequate design of the asphalt layer as well as inappropriate bond between the layers or even loss of bond (Rao, 2013).

In the case of composite pavements, rutting is a more complex issue due to the presence of the rigid base under the layer of asphalt. Because of the high stiffness of the base, the asphalt layer absorbs most of the deformations due to vertical strains (Hernando and del Val, 2013). However, the presence of a rigid base instead of an unbound aggregate base can play a significant role in the development of rutting, because the permanent deformations develop in the much less stiff asphalt (Rao, 2013).

Researchers have dealt with the design of a composite pavement with the main aim being able to estimate the appropriate asphalt overlay thickness required to carry the design traffic. Other researchers investigated the main factors that affect the development of reflective cracking in these pavements with the help of methods like modelling with finite element or with laboratory experiments or even with real data from built composite pavements. A limited number of the models tried to predict the appearance of reflective cracks by taking into account traffic and thermal loading.

\subsubsection{Reflection cracking}

Reflection cracking is a unique type of distress found in composite pavements whereby cracks grow in the asphalt surfacing or overlay immediately above discontinuities (cracks or joints) in the underlying concrete layer (Mallick \& El-Korhi, 2013). Reflective cracking is a complex mechanism and can be due to the combined effects of traffic and thermal loading and the likelihood of it occurring has been found to be strongly dependent on the amount of movement at the joint or crack in the concrete layer (Vanelstraete \& Francken, 1997). Reflective cracking can either occur in one cycle or over many cycles depending on the situation (Wright, 2009) and the role of existing flaws and discontinuities in the pavement has been found to be significant (Elseifi \& Al-Qadi, 2004). To further complicate the situation, reflective cracking has been found to initiate either at the top or the bottom of the asphalt layer and propagate through the material (Scarpas \& de Bondt ,1996; Elseifi et al., 2004; Molenaar \& Pu, 2008; Nesnas, 2004). 
The appearance of reflection cracks on the surface of the asphalt overlay is not immediately connected to a failure of the pavement. However, depending on the severity level of the cracks (low, medium, high), in the case of them being unsealed, water can infiltrate and deteriorate the condition of the pavement in the inner layers creating deficiencies to the structural ability of the pavement. In addition, the number of cracks on the surface results in the increase of the pavements roughness reducing its effective serviceability. In Parry et al. (1999) it is mentioned however that the appearance of reflective cracks is not immediately connected with a quick deterioration of the pavements and that in some studies no correlation was found between the number of reflective cracks and pavement deterioration (Mayhew and Potter, 1986).

To be able to predict the performance of a pavement, in regards to reflective cracking, it is important to consider movements due to actual loading conditions like traffic and climatic. During the passage of a wheel, three pulses of stress are typically experienced in the pavement and particularly at the tip of the crack as it progresses through the overlay. These pulses are one bending stress when the wheel is over the crack and two shear stresses, one before the wheel passes the crack and one after (Kohale and Lytton, 2000). This can be seen clearly in the following figure, where according to the position of the wheel load on the pavement in relation to the crack, bending and shearing stresses develop. The bending stress is highest when the wheel is on top of the crack, and the shearing stresses are maximum when the wheel load is either side of the crack. This gives an idea to where the critical positions of a vehicle load may be for the development of reflective cracking. Due to seasonal temperature cycles or daily temperature cycles, the layers of the pavement can contract or expand, leading to excessive tensile stresses in the area above the pre-existing crack or joint of the underlying layer (Mukhtar and Dempsey, 1996; Kohale and Lytton, 2000; Pais et al, 2000). This results in the crack developing through the asphalt overlay (Mukhtar and Dempsey, 1996). 


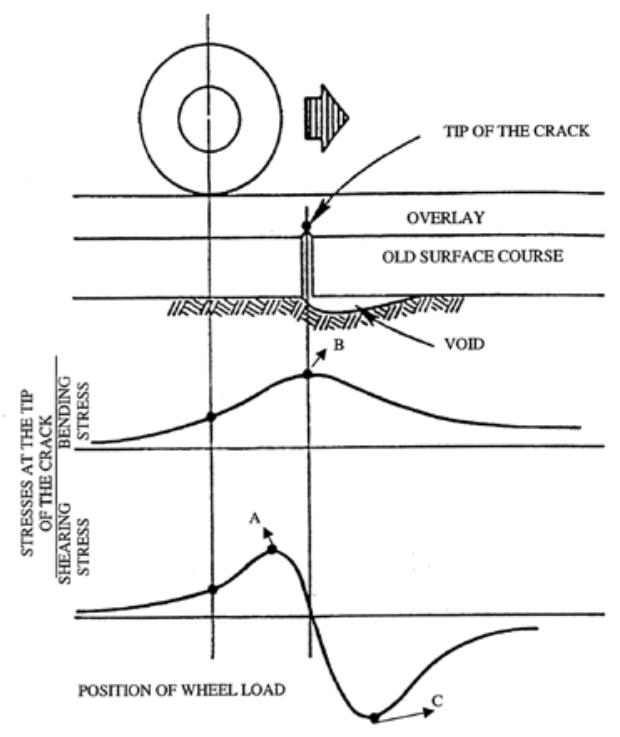

Figure 1: Stresses in the asphalt overlay due to a wheel load. (Kohale and Lytton, 2000)

Reflective cracking can propagate in two ways, either from the bottom of the overlay upwards or in the opposite direction, from the top to the bottom. Researchers like Scarpas \& de Bondt (1996), Elseifi et al. (2004) and Molenaar and Pu (2008) have supported the view that reflection cracking occurs only from the bottom to the top, whereas others like Nesnas (2004) state that it occurs from the top only. Both types although have been noticed and confirmed and should be considered.

Wright (2009) found that reflection cracks develop both from the bottom and from the top of the asphalt layer depending on the load transfer efficiency and the differential deflections of the existing joint or crack in the underlying layer. It was also found that the thickness and stiffness modulus of the asphalt, as well as whether full bonding existed between the asphalt and the underlying layer, were important factors in determining whether reflection cracks initiated or not.

A study by Nesnas \& Nunn (2004) supported the view that reflective cracking occurs only from the top of the asphalt layer in as-laid composite pavements. Their approach considered the viscoelastic behaviour of the asphalt and the fact that it can age during its service life. The annual temperature variations as well as the diurnal variations between day and night, can cause high thermal stresses nearer the surface of the asphalt layer due to its contraction. The area of the asphalt surface was also found to be more prone to ageing which can result in a reduction of the asphalt's stress relaxation ability. Therefore, the ability of the asphalt to accommodate cracks at the surface of the layer would 
be reduced, and Nesnas \& Nunn (2004) argue that the appearance of cracking on the top of the asphalt is more probable.

\subsection{Research objectives}

This research project aims to develop a validated model which can be used to predict distresses in flexible composite pavements over a long period of time due to both traffic and environmental loading. The model will be the first of its kind to include factors such as dynamic vehicle loading in modelling the long-term performance of flexible composite pavement structures. The idea for its development originated on pre-existing research on a long-term flexible pavement performance. The goals is to incorporate it in a vehicle-pavement interaction software.

Specifically, the research objective is to develop a model for composite pavements that with the help of measured data and a vehicle dynamic loading model would be used to predict the distress of reflective cracking. This is done with the help of a simplified mechanics-based model in combination with an existing vehicle dynamic model. The goal is to model both traffic and thermal driven reflective cracking.

In addition to that, the identification of parameters which affect the behaviour of a composite pavement, that could be among others structural parameters or loading parameters is aimed. The conclusions can be used in the development of design methods of a composite pavement.

\subsection{Research approach}

The first step was to research into the availability of data on composite pavements that could be used as a form of validation of the model. This data was found in the Long-Term Pavement Performance database where information about pavement sections over the USA are given regarding the original construction of pavement sections, the climatic conditions, the pavement performance and the traffic axle data.

After identifying the pavement sections that could be used in the research study, a simplified mechanistic model that was developed at Nottingham University by $\mathrm{N}$. Thom was identified as the ideal model upon which the new research could be based. 


\subsection{Thesis structure - Research scope}

The thesis includes eight chapters. The first chapter is the introduction to the thesis topic and objectives. This chapter also includes the general background about composite pavements and a thorough literature review regarding the matter of reflective cracking which is the main distress in composite pavements.

In the second chapter the model selected to analyse reflective cracking is presented and the way it works is investigated in further detail by focusing on the two main sub-models that regards bottomup cracking and top-down cracking. Also, some example simulations are included in order to understand the way the program works, and the effect of important parameters is investigated. In the third chapter, the thermal cracking model is presented, describing the different sub models that it consists of as well as the effects of important parameters.

The fourth chapter is used to present the dynamic vehicle model found in literature and adapted for this study, as well as the way it is incorporated here.

In the following chapter, chapter 5, the whole life long term model is presented. The basic framework is presented, along with a flowchart to aid visually in understanding the way the whole model works. A simulation of an example pavement section is also presented step by step until the final outcome.

The $6^{\text {th }}$ chapter includes a convergence study with the help of which the basic time elements of the whole model were selected, as well as a parametric study of the basic parameters of the model, in order to identify the sensitivity of the results to the variation of these parameters.

In the $7^{\text {th }}$ chapter, the data from the Long-Term Pavement Performance database used for validation is presented, followed by the validation analysis which includes simulations of real pavement sections along with predictions of failure for each section.

The final chapter is a general summary of thesis, including conclusions from each chapter as well as general regarding this research study. Also, recommendations for future work are presented. 


\section{Modelling of reflective cracking}

\subsection{Review of previous work}

In general, there are different ways with which the response of a pavement can be modelled. For example, the main structural layers of the pavement can be represented as a beam, or a plate, with elastic or viscoelastic properties. The soil structure underlying the pavement can be modelled as an elastic foundation characterized by a modulus of subgrade reaction or a layer with elastic springs and dashpots. The load can either be concentrated or a distributed load over a specific length (Akbarian, 2012).

More specifically, in regards to composite pavement modelling, a number of research studies focus on the design of a composite pavement and the estimation of an appropriate asphalt overlay thickness (Cho et al., 1998; Sousa et al., 2002; Kohale and Lytton, 2000; Minhoto et al., 2008). Efforts have also been made to predict the overlay life or the life of a composite pavement, usually by calculating the number of wheel loads to failure with the help of a crack propagation law. Some examples of this sort of research can be found in OLCRACK Thom (2000), Ullidtz et al., (2010), Lytton (2010), Owusu-Antwi et al. (1998), Elseifi \& Al-Qadi (2004), Scarpas \& de Bondt (1996), Zhou et al (2010).

Various methods of modelling the complex mechanisms associated with reflective cracking in pavements have been identified from the literature. These include empirical models, multilayer linear elastic models, models based on equilibrium equations and finite element analyses with or without the use of fracture mechanics (e.g. Seeds et al. 1985, Thom 2000, Scarpas \& de Bondt 1996).

\subsubsection{Simple mechanistic models}

A model of this type was developed by Austin Research Engineers as stated by Seeds et al. (1985). Two basic reflective cracking failure modes were assumed the first being an opening failure mode due to horizontal movement of the concrete slabs, and the second a shearing mode due to shear in the areas of the cracks. The horizontal movements are attributed to the temperature variations and the vertical movements are due to differential deflections occurring at the cracks caused by wheel loading. These movements can also be depicted in Figure 2 from Seeds et al. (1983). This model is used to define the number of single axle loads the pavement can accept before failing due to reflective 
cracking, which is done with the help of a fatigue approach. This model combines the two main causes of reflective cracking by considering shear strains due to vehicle loads and tensile strains due to thermal variations, but it is calibrated only for the area of Arkansas.

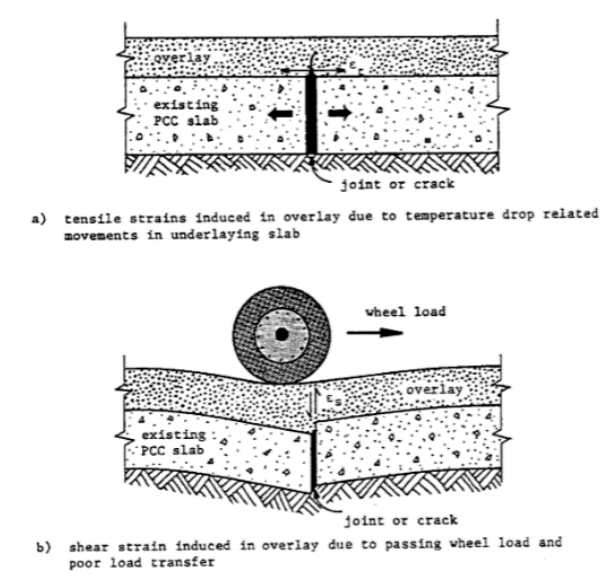

Figure 2: Distress mechanisms. Seeds et al. (1983)

OLCRACK was originally developed by Thom (2000) to study the influence of grid reinforcement in composite pavement structures as a mitigation technique against cracking. This model predicts crack growth both from the surface and from the base of the asphalt layer in this specific type of pavement. A fatigue approach was used for the calculation of a crack propagation rate which is driven by the tensile strain in the region of the crack. The materials were characterized by stiffness moduli and simplified equations of statics and mechanics were used to model the cracking mechanisms in the pavement structure. Results showed that the model was able to predict cracking from both the base and the surface of the asphalt layer. An example of the output of the program is shown in Figure 3 where the reinforced case is compared with the unreinforced. The predicted overlay life is longer for the reinforced case. 


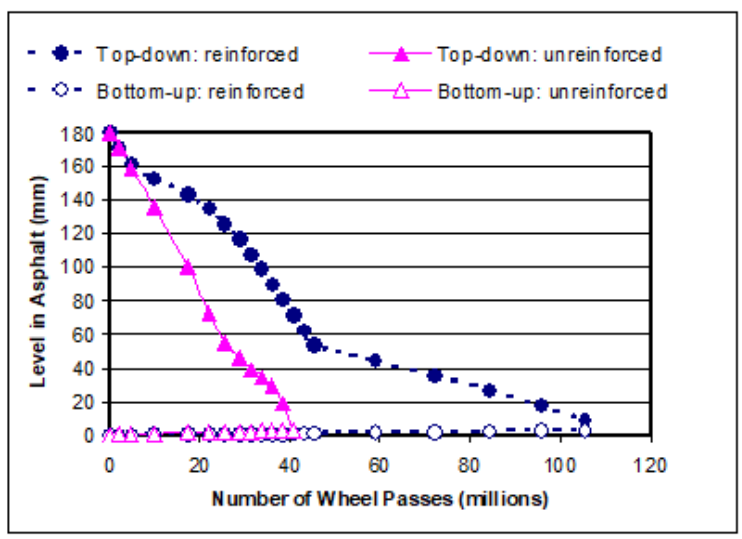

Figure 3: Prediction of overlay life. Thom (2000)

\subsubsection{Finite element analysis (with or without fracture mechanics)}

Finite element analysis is a very common way to model pavements with layers of different composition, and specifically pavements which develop discrete cracks. Typically, these models are used to calculate tensile stresses and strains in the asphalt layers which are then either combined directly with an empirical fatigue relationship or via a fracture mechanics approach to estimate crack growth and the life of the pavement.

Sousa et al. (2002) created a mechanistic-empirical overlay design method for composite pavements to resist reflective cracking by taking into account factors like age, temperature and field performance. After implementing finite element analyses to calculate the shear stresses in the area of a crack, a statistical model was developed following laboratory testing. This model aims to calculate the overlay asphalt thickness which is required, to avoid the appearance of reflective cracks, after the loading of Equivalent Single Axle Loads (ESAL). A constraint in using this model is the fact that crack propagation is not taken into account (NCHRP, 2010). The generalised model has been calibrated with measurements from a specific area of the US and also information like existing cracks and deflection history are required.

A computer program that progresses from empirical modelling to mechanistic-empirical is CalME, developed by the California Department of Transportation (Caltrans) (Ullidtz et al., 2010). It was developed for the analysis and design of new flexible pavements or existing pavement rehabilitation. An incremental - recursive procedure can be used to predict the performance of a certain design of pavement. A method by Wu and Harvey (2012) is used to calculate the reflective cracking. In this 
method the tensile strain at the bottom of the asphalt overlay is used to calculate the damage in the asphalt. This tensile strain depends on many factors including layer thicknesses, moduli and crack spacing. Reflective cracking density is then calculated with the help of a regression equation that includes the previous factors as well as the modulus of the damaged asphalt material. The results showed that the predictions not always match the measured pavement responses, due to errors or neglecting certain parameters like temperature effect on the fatigue of the asphalt.

Very thorough research has been completed within the National Cooperative Highway Research Program (Lytton, 2010) where a mechanistic model for reflective cracking predictions on pavement sections was developed. The goal for this model was to be included in the MEPDG software as a subprogram. It was based on finite element modelling along with the help of fracture mechanics based on the Paris crack propagation law. The three different mechanisms of crack propagation, thermal, traffic bending, and traffic shearing were taken into account to develop a regression model based on 5 different parameters. The first parameter is the number of days required so that the crack developing would reach the critical position where bending stresses become compressive and no longer contribute to crack development $\left(\mathrm{N}_{\mathrm{fB} 1}\right)$. At that point, the other two parameters are calculated which are the number of days required for crack growth up to the first position due to shearing stresses ( $\left.\mathrm{N}_{\mathrm{fs} 1}\right)$ and thermal stresses $\left(\mathrm{N}_{\mathrm{fT} 1}\right)$. The two remaining parameters are the number of days required when the crack from the first position reaches the top of the overlay due to shear stresses $\left(\mathrm{N}_{\mathrm{fs} 2}\right)$ and thermal stresses ( $\left.\mathrm{N}_{\mathrm{fT2}}\right)$. These five parameters are included in the final calibration equations. They can also be seen in Figure 4, where the equivalent stresses are shown along with the relevant number of days required for crack growth due to each mechanism, Bending $\left(\mathrm{N}_{\mathrm{fB} 1}\right)$, Shearing $\left(\mathrm{N}_{\mathrm{fS} 1}, \mathrm{~N}_{\mathrm{fS} 2}\right)$ and Thermal $\left(\mathrm{N}_{\mathrm{fT} 1}, \mathrm{~N}_{\mathrm{fT} 2}\right)$ for position 1 and position 2.

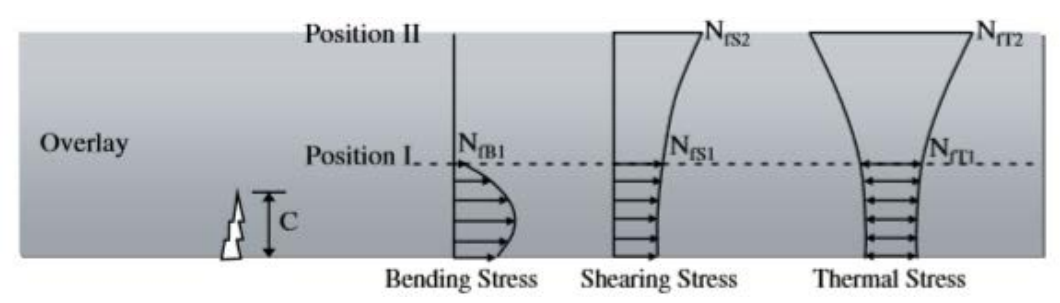

Figure 4: Number of days of crack growth. (Lytton, 2010) 
The Mechanistic-Empirical Pavement Design Guide (MEPDG) was developed as a tool based on mechanistic and empirical principles and is used to predict the state of pavements by using performance indicators. These consist of transfer functions and regression equations which were calibrated with data from the LTPP (Long-Term Pavement Performance) database. According to a webinar from the Transportation Research Board, (Quintus, 2016) the newest reflection cracking integration in MEPDG software has the form of

$$
R C R_{i}=C\left(\frac{100}{c_{4}+e^{c_{5}\left(\log \left(D I_{i}\right)\right)}}\right)
$$

Where RCRi: Reflective cracks

C: Transverse cracking in underlying pavement layers

c4, c5: Calibration factors

DIi: Damage ratio

and it can be used to calculate fatigue cracks and transverse cracks, representing a purely empirical way to estimate reflective cracks.

Other models are based on finite elements analysis in combination with fracture mechanics, to account for the growth of the crack in the pavement after the initiation phase. The goal of OwusuAntwi et al. (1998), was to develop a mechanistic model that would predict reflective cracking on asphalt overlays. With the use of finite element analysis to calculate J-integrals they used fracture mechanics to calculate the number of loads needed to failure and investigate the crack propagation. With the help of damage mechanics and calibration with data from LTPP GPS 7 experiment the total damage from traffic and temperature was related to the total number of reflective cracks in the form of a mechanistic-empirical equation. The actual traffic load distribution was used which is better than using only one weight load. The basic factors studied were number of axle load applications, age, and asphalt overlay thickness.

On a similar note, Scarpas \& de Bondt (1996) developed a fracture mechanics-based model for pavements overlaid with asphalt. It was incorporated into the finite element program CAPA to investigate the contribution of grid reinforcement of the asphalt to the life of a composite pavement. The crack propagation is simulated by starting with an initial crack length, that propagates through 
the thickness of the asphalt. The reinforcement was found to be a good anti-reflective cracking measure if firmly attached to the existing pavement surface prior to placement of the overlay. Using this approach, they were able to simulate the growth of discrete cracks in the asphalt pavement.

An effort to predict the fatigue life of a composite pavement reinforced with an interlayer was made by Doh et al. (2009), and the thought was to modify the crack growth rate in the classic Paris law fatigue life equation. Crack growth rate $(\mathrm{da} / \mathrm{dN})$ was replaced by horizontal deformation rate $(\mathrm{du} / \mathrm{dN})$. The reason behind this was due to the interlayer it would not be reliable to use the fatigue law as is due to the layers not being continuous. The progression of reflective cracking was based on the concept of bending fracture. It was shown that this prediction model could be used to predict the fatigue lives of composite pavements with interlayers. This conclusion was reached after comparing results of the model with laboratory test data.

Elseifi \& Al-Qadi (2004) investigated reflective cracking due to traffic loading by using a 3D finite element analysis and fracture mechanics. They tried to predict the overlay life of a rehabilitated pavement structure by developing a model which could predict number of cycles to reflective cracking failure for rehabilitated flexible pavement structures (HMA overlay over cracked asphalt pavement in this case). They recognised two distinct phases, the crack initiation process which was related to the shear strain developing at the cracks followed by the crack propagation stage. To simplify calculations, they developed a regression equation that takes into account factors like the thickness and modulus of the overlay, and the properties of the base, subbase and subgrade and estimates the total number of $80-\mathrm{kN}$ single axle loads according to a specific design of pavement. They found that the overlay thickness and the thickness of the existing asphalt layer were the major factors affecting the life of the overlay.

Several researchers investigated the effect of the deflections and load transfer efficiency on the development of reflective cracking like Wright, (2009) and Seeds, (1985). In a study by Zhou \& Sun (2000) a finite element analysis approach was used to model cracking in composite pavements. They found that, depending on the thickness of the asphalt, reflective cracks appeared in either pairs or as a single crack. Fracture mechanics was used to study the crack propagation and Zhou \& Sun (2000) found that the relative deflection between the two sides of the joint in the underlying layer was 
important for crack propagation due to both bending and shearing effects. The deflection on the loaded side of the joint was the one that contributed to the initiation of a crack due to bending, and the relative deflection between the two sides contributed only to the propagation of the crack due to bending and shearing effects.

Reflective cracks have also been encountered in airfield pavements. According to Garzon et al. (2010), the prediction and simulation of these is based on 3D models. These use the Generalized Finite Element Method (GFEM) instead of the standard (FEM). This method enables the modelling of the actual load distribution with no need for two-dimensional simplifications and can predict the direction of the crack.

A study based on 3D finite element analysis by Kuo \& Hsu (2003) shows the development of both bottom-up and top-down cracking. The development of the latter occurs especially in the occasion of higher temperatures when the asphalt layer becomes softer or when the asphalt layer is in general thicker and has as a result the reduction of stresses developing at the bottom of the layer. The effect of a geogrid as a mitigation technique of reflective cracking was also studied and it was seen that it can postpone reflective cracking appearance, depending on its strength. The placement of the geogrid can separate the asphalt layer in a lower layer that ensures efficient bonding of the interlayer and the upper layer. It also helps in an even distribution of deformation energy between the upper and lower layer and therefore, the whole thickness of the asphalt layer. Fatigue life prediction was used as a technique for the actual prediction of the cracking path.

\subsection{Olcrack - Theoretical modelling}

This chapter presents the theoretical background to the approach used to model reflective cracking in flexible composite pavements due to vehicular loading. The model is based on research undertaken by Thom (Thom, 2010) at the University of Nottingham, who developed a relatively simple approach based on equilibrium mechanics to predict the rate of cracking through a composite pavement containing grid reinforcement. In this research, the effects of the grid were eliminated, and the model was extended by integrating it with a simple dynamic vehicle model to predict the progression of reflective cracking throughout the life of the pavement. The model was implemented using MATLAB 
software. Key elements of the model are summarised in this chapter, a full description can be found in Thom (1990) ${ }^{1}$.

Figure 5 shows the idealised composite pavement structure used for the modelling. It comprises an asphalt layer of thickness 100mm over a cracked concrete layer (in this case in three slabs, A, B and C) of thickness $200 \mathrm{~mm}$ over a subbase of thickness $250 \mathrm{~mm}$ over a semi-infinite subgrade. It is assumed that all materials are linear elastic characterised by an elastic stiffness modulus and Poisson's ratio. Note that in the case of the asphalt layer the stiffness modulus is dependent on temperature and loading time as asphalt can be idealised as a viscoelastic material.

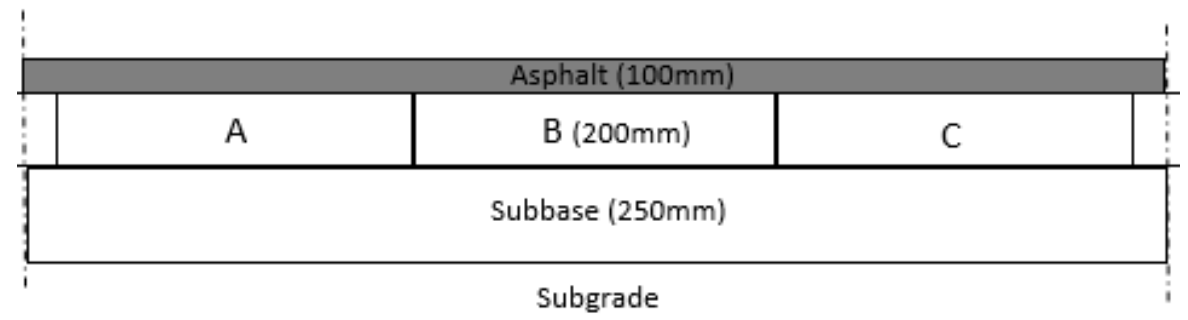

Figure 5: General structure of composite pavements.

The main goal of the modelling is to predict reflective cracking in the asphalt overlay, directly above the locations where the underlying layer has the pre-existing cracks or joints. Two separate situations are considered regarding the position of crack initiation, from the bottom of the asphalt layer or from the top of the asphalt layer. These two situations are analysed separately and are found to depend on different critical conditions which include the position of the wheel load in relation to the horizontal distance from the crack in the underlying layer.

In the approach used, it is assumed that the crack can potentially initiate after the first application of a wheel load and the development of the crack progresses through the thickness of the asphalt as additional wheel loads are applied. The crack propagation rate for bottom-up and top-down cracking both depend on a power law relationship with the tensile strains calculated in the region of the crack tip. The crack propagation rate, in combination with the number of wheel loads trafficking the

\footnotetext{
${ }^{1}$ Personal correspondence with N. Thom, Nottingham University, UK from 2014-2018, based on unpublished research work from 1990
} 
pavement after a selected time increment, are used to calculate the increment in crack development through the asphalt layer. The mechanics of both situations are presented in the following sections.

\subsubsection{Bottom-up cracking mechanism}

In the bottom-up situation, cracking initiates from the base of the asphalt layer and progresses towards the pavement surface. The critical situation is considered to be when the wheel load is directly above the joint or crack in in the underlying layer, as can be seen in Figure 6. When the wheel is at this position, the asphalt is forced to bend, and tension is created in the area at the bottom of the asphalt layer. An idealised structure of the pavement in this specific situation is shown in Figure 6 and is taken from previous work of Thom (2000).

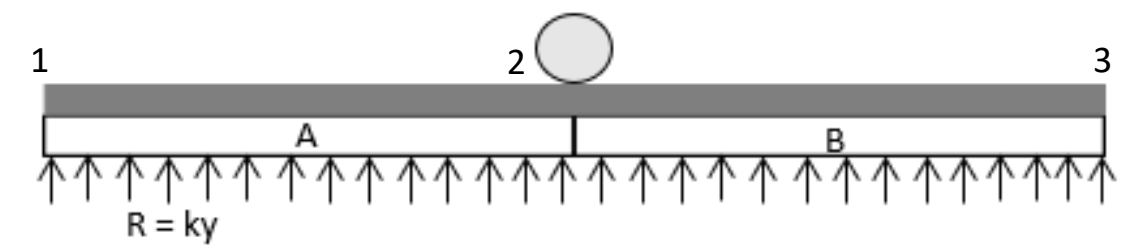

Figure 6: Bottom-up cracking model.

The asphalt layer is modelled as a continuous elastic beam. The cracked base of the pavement is considered to be rigid and comprises two slabs, A and B, which are hinged at points 1 and 3, so that the slabs can rotate about these points. The foundation is characterized by a modulus of subgrade reaction $(\mathrm{k})$, which represents the support of the underlying layers.

When the wheel is directly above the joint between the two slabs, the whole structure at that point is assumed to deflect vertically by an amount y1. This results in the underlying slabs deflecting at that point and rotating about their hinged ends. Due to this rigid body mechanism there will be a loss of contact between the base of the asphalt and the underlying slabs in the vicinity of the load. The distance to which this extends to either side of the loading point is referred to as the debonded length and is shown schematically in Figure 7. The debonding of the two layers results in a redistribution of the stresses through the thickness of the asphalt. From the bending of the asphalt, the stress distribution can be calculated when the crack appears and the strain in the region of the crack tip can also be estimated using the following procedure which is summarised below and described in detail in Thom (1990). 


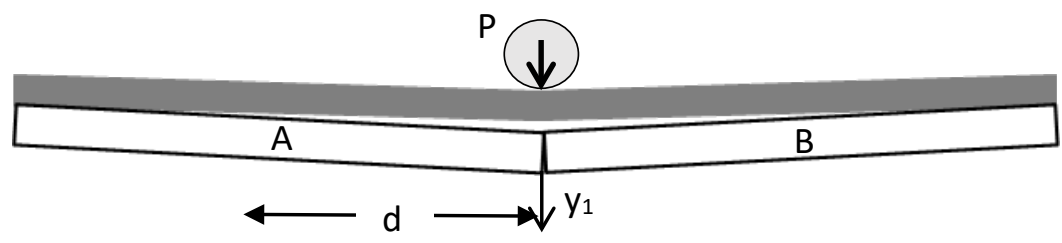

Figure 7: Bending in asphalt and slab deflection.

Figure 8 shows a flowchart of the procedure followed to estimate bottom-up cracking. After the application of a wheel load $\mathrm{P}$, above a joint on the surface of the asphalt layer of certain elastic modulus E, the pavement layers deflect by a deflection of y1. This creates the debonding effect between the two layers described above and a certain bending moment in the asphalt. This bending moment will lead to a strain in the vicinity of the crack tip and, with the help of a fatigue law, the crack propagation rate is calculated. Depending on the number of wheel loads traversing the pavement the crack length of the first-time increment is calculated. 


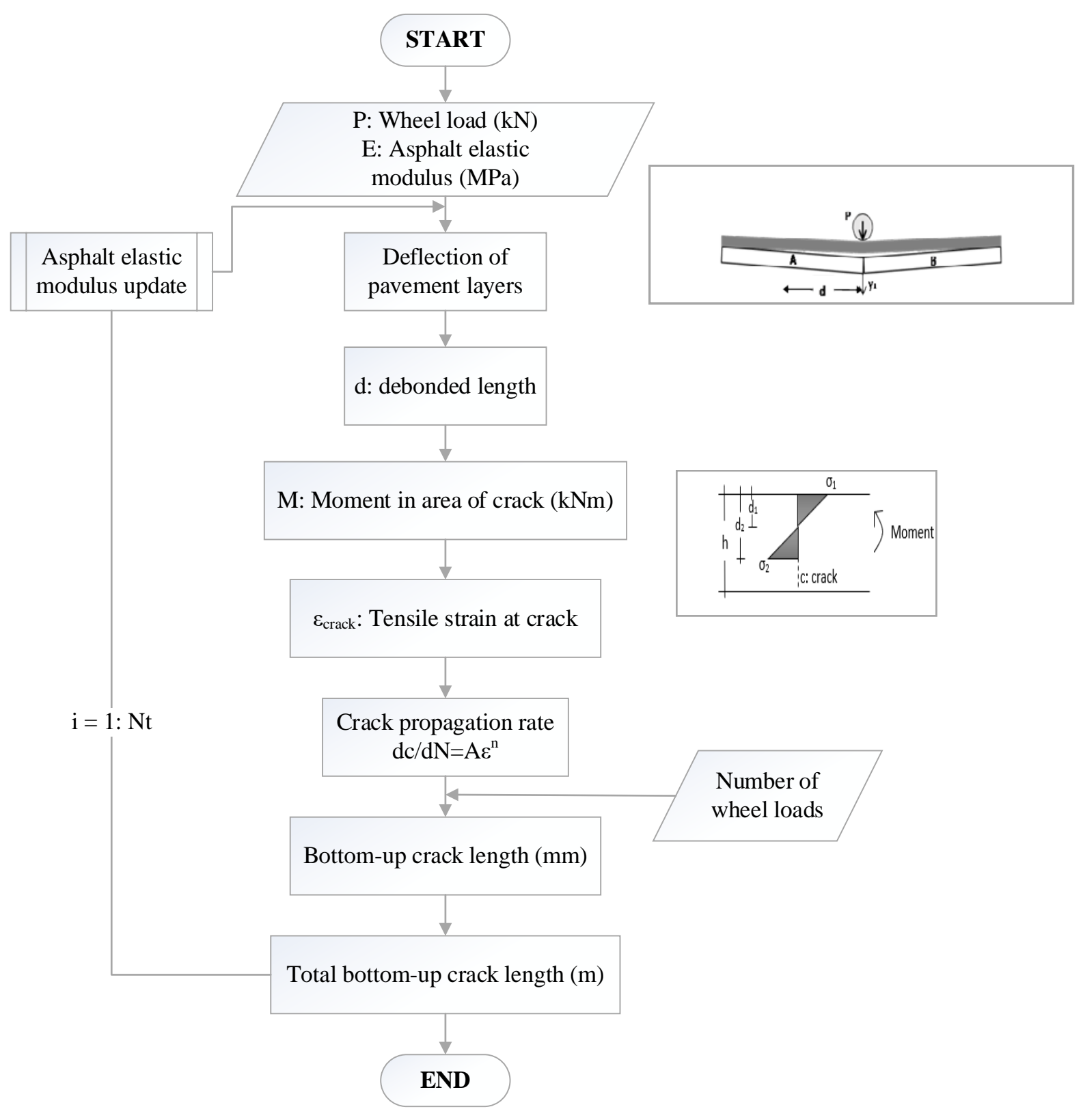

Figure 8: Flow chart for bottom-up cracking mechanism.

With the appearance of a crack in the asphalt overlay, the elastic modulus of the asphalt is affected, and its original stiffness is reduced for the next iteration.

It is significant to notice that the way the program is built, it assumes that an infinitesimal crack will be created after the first-time increment, when initially the crack is 0 . Therefore, it automatically assumes that the stress developing in the asphalt is enough to overcome the breaking strength of the 
asphalt and eventually create the initial crack. In regards to the propagation, after each time increment a new crack is calculated which is cumulatively added on to the previous one and the total crack length is updated. The bottom-up cracking procedure ends in one of two cases: the crack developing from bottom-up meets the top-down crack and no further crack development is allowed either way, or a pre-specified number of wheel loads corresponding to certain duration of time have all passed.

Further information is given below regarding:

- the debonded length,

- the bending strain that develops in the bottom-up cracking mechanism,

- the crack propagation rate and crack length calculation, and

- the reduction of the asphalt elastic modulus.

Thom (2000) used compatibility conditions between the upper and lower layers to estimate the debonded length, which he found to be given by the solution to the following quadratic equation:

$$
\mathrm{P} \frac{\mathrm{d}^{2}}{4}+\frac{3}{2} \frac{\mathrm{EIP}}{\mathrm{kL}^{3}} \mathrm{~d}-\frac{3}{2} \frac{\mathrm{EIP}}{\mathrm{kL}^{2}}=0
$$

Where:

P: wheel load, $\mathrm{kN}$

d: debonded length, $m$

E: asphalt elastic modulus, MPa

I: moment of inertia of the asphalt beam, $\mathrm{m}^{4}$

k: subgrade of elastic modulus, $\mathrm{MN} / \mathrm{m}^{3}$

L: length of the slab, $m$

\section{Bending strain at crack}

Due to appearance of a crack in the asphalt layer, the stress distribution will be modified due to the crack being unable to resist tensile forces. Consequently, the remaining thickness of the asphalt undergoes bending, and therefore no tensile stress is distributed over the length of the crack as shown in Figure 9. 


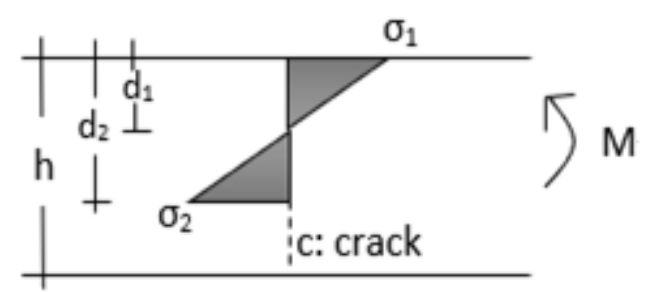

Figure 9: Stress distribution with bottom crack in asphalt.

The force balance between the stresses $\sigma_{1}$ and $\sigma_{2}$ as well as the moment balance due to the bending moment $\mathrm{M}$ and the moments from the stresses are used to identify the tensile bending stress distribution ( $\sigma_{1}$ and $\sigma_{2}$ ). This is achieved assuming that the neutral axis is midway between the upper surface of the beam and the crack tip. In the original work by Thom (1990) this was more complicated as the forces from reinforcement were also included. In this case after combining the balance equations the bending stress at the crack is given by:

$$
\sigma_{2}=\frac{3}{2} \frac{M}{d_{1}^{2}}
$$

Where according to calculations of Thom (1990) M is given by:

$$
M=\frac{P}{4}(2 d-r)
$$

and

$$
\mathrm{M}=\frac{\mathrm{Pd}^{2}}{4 \mathrm{r}}
$$

depending on whether the radius of the contact patch (r) is smaller or larger than the debonded length respectively. The equivalent maximum tensile bending strain at crack will be given by:

$$
\varepsilon_{\mathrm{t}}=\frac{\sigma_{2}}{\mathrm{E}}
$$

\section{Crack propagation rate and crack length calculation}

Progression of the crack through the asphalt layer is based on a fatigue law where the crack propagation rate is assumed proportional to the power of the strain near the crack tip (Thom, 2014): 


$$
\frac{\mathrm{dc}}{\mathrm{dN}}=A \varepsilon_{\mathrm{t}}^{\mathrm{n}}
$$

A and $\mathrm{n}$ are constants which can typically be calculated for asphalt from fatigue tests such as the indirect tensile fatigue test (Brown et al., 2001). The crack propagation rate is then multiplied by the number of wheel loads that have traversed the pavement section within that time increment to give the amount the crack has grown:

$$
\mathrm{C}_{\mathrm{bu}}=\frac{\mathrm{dc}}{\mathrm{dN}} \times \mathrm{N}
$$

Where $\frac{\mathrm{dc}}{\mathrm{dN}}$ the crack propagation rate, $\mathrm{N}$ the number of wheel loads and $\mathrm{C}_{\mathrm{bu}}$ the length of the crack development from bottom to up.

\section{Update of asphalt elastic modulus after time increment}

As can be seen from the flowchart in Figure 8, as the bottom-up crack grows, the effective modulus of the asphalt is reduced effectively accelerating the cracking process. This can be thought of as a positive feedback mechanism and this sub-section describes how the effective modulus of the asphalt is calculated.

To explain that visually, the presence of a crack can be idealised as a "dead zone" as shown in Figure 10 (grey area) (Brown, 2001). This zone is considered as stress free zone and the thickness of the asphalt layer is effectively reduced $\left(\mathrm{h}-\mathrm{C}_{\mathrm{bu}}\right.$ ). The distance at which the crack does not affect the asphalt layer should be identified. The length of the dead zone on each side of the crack is therefore represented by the destressed length ( $t)$, and the procedure for its definition is briefly described in Brown et al. (2001) with the help of the rectangle of the affected beam area adjacent to the crack.

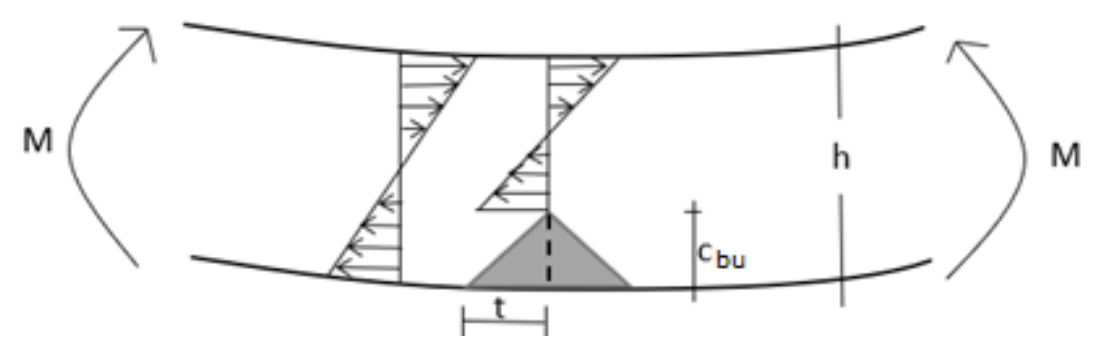

Figure 10: Stress distribution and dead zone due to crack. 
Thom (1990) estimated that the destressed length is given by:

$$
t=\sqrt{\left(8 C_{b u}^{2}(1+v)\left(\frac{h}{4 C_{b u}}-\frac{1}{6}\right)\right)}
$$

where $\mathrm{v}$ is Poisson's ratio.

After a number of load repetitions, which create a certain crack growth, a new effective asphalt modulus is calculated to reflect the reduction of the asphalt stiffness due to the crack (Thom, 2014). These calculations take into account a reduction in the asphalt's stiffness or an equivalent increase in its compliance (noting that compliance is the inverse of stiffness).

To visualize that, in Figure 11, the length of the asphalt which is under bending is shown, assuming that this covers only the areas of the debonded length ( $L=2 \times d$ ) over the two adjacent slabs and not the whole length of the slabs, as this would simplify calculations. The beam under examination in Figure 11 is divided in 3 parts, which are described by a different compliance. Areas 1 and 3 are considered as typical cases of a beam on elastic foundation, with no cracks, characterised by a compliance of $\mathrm{C}_{1}$. Area 2 is affected by the no-stress zone and is represented by a beam in bending under a moment $\mathrm{M}$, with a reduced effective asphalt modulus Eeff and a compliance of $\mathrm{C}_{2}$.

The bending stiffness for a simple beam is proportional to $\mathrm{Eh}^{3}$ (where $\mathrm{E}$ is the modulus and $\mathrm{h}$ is the thickness) and so, for a material modulus, the compliance of the beam can be considered proportional to the inverse of the cube of the thickness $\left(1 / h^{3}\right)$ for a given modulus.

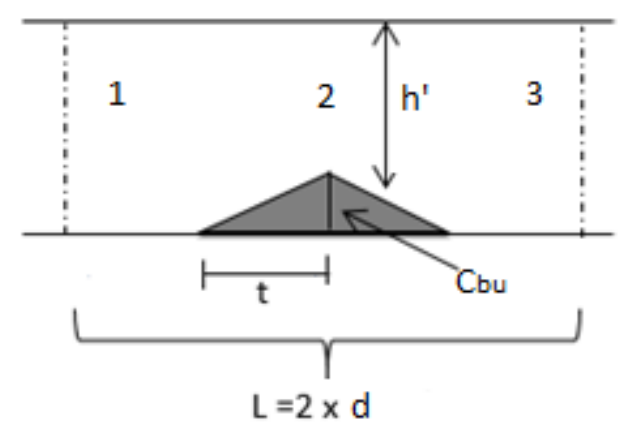

Figure 11: Dead zone in asphalt due to bottom crack.

So, the total compliance of the asphalt is calculated according to: 


$$
\frac{1}{\mathrm{~L}}\left[\mathrm{C}_{1}(\mathrm{~L}-2 \mathrm{t})+2 \int_{0}^{\mathrm{t}} \mathrm{C}_{2}\right]
$$

But because the compliance is the inverse of the asphalt stiffness $\mathrm{S}=\mathrm{Eh}^{3}$ the compliances $\mathrm{C}_{1}$ and $\mathrm{C}_{2}$ will be proportional to the inverse of the cubed thickness of the asphalt for each area. These assumptions would lead to the effective modulus of the asphalt (Eeff) which was shown by Thom et al. (1999) to be given by:

$$
E_{e f f}=\frac{E}{\left[\frac{L-2 t}{L h^{3}}-\frac{t}{L C_{b u} h^{2}}+\frac{t}{L C_{b u}\left(h-C_{b u}\right)^{2}}\right] h^{3}}
$$

\subsubsection{Top-down cracking mechanism}

The idealized model that is used for top-down cracking is shown in Figure 12 and it consists of three rigid slabs A, B and C. Similarly to the bottom-up model, the ends of slabs A and C are hinged, and the foundation is modelled using a modulus of subgrade reaction. However, the mechanism is more complex.

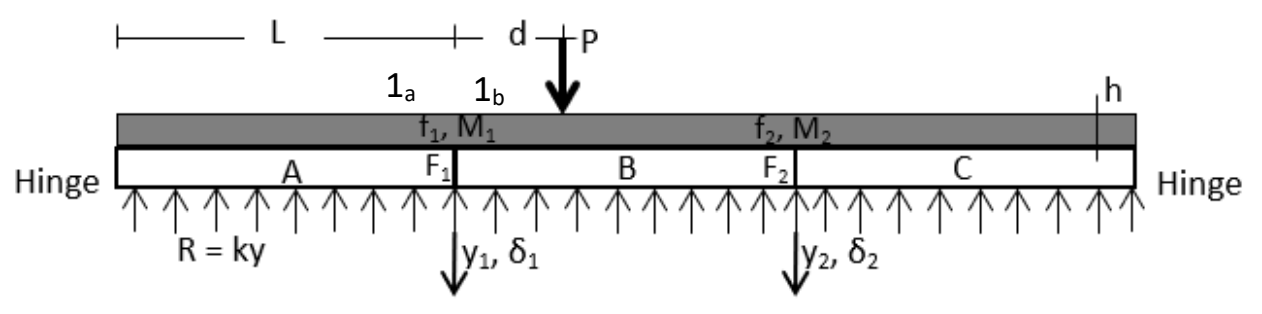

Figure 12: Top-down cracking mechanism.

When the wheel load (P) passes over the first crack in the underlying layer (point 1), the asphalt flexes. As the wheel continues along the pavement and the distance from the first crack grows, the left-hand end of slab B tends to detach from the asphalt in that area as is shown in Figure 13. A differential movement takes place between slabs A and B and the asphalt is forced to bend coming down from slab A to where slab B has moved due to the load, causing differential deflections $\delta_{1}$ and a deflection at the end of slab A, $\mathrm{y}_{1}$. It is assumed that there is no resistance to the separation between the asphalt and the slabs (Thom, 2000). 


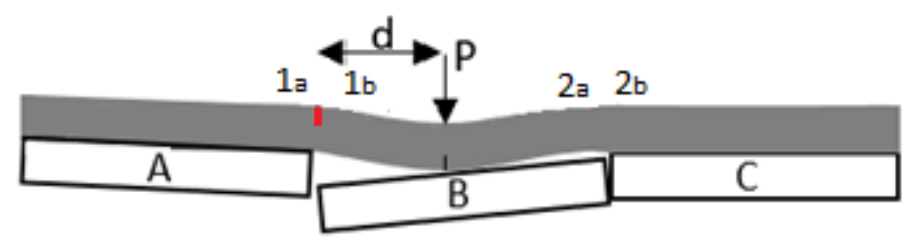

Figure 13: Top-down cracking mechanism due to wheel load.

The loading on the asphalt results in a displacement of the layer at that point of loading. The displacement is maximum vertically at that point and decreases towards each end of the slab either side of the load. This happens because, the vertical movement of the area above the underlying crack at point $1_{\mathrm{a}}$, is restricted due to the support from the right-hand end of slab $\mathrm{A}$. This has as a result the development of tension as can be seen from the idealized model in Figure 13, in the top area of the asphalt at point $1_{\mathrm{a}}$ where there is a resistance to vertical displacement. It is important to estimate the bending moment in the area as well as the forces that appear. Consecutive loading points of the wheel are considered along the pavement, and the moment at location $1_{\mathrm{a}}$ due to each of them is calculated $\left(\mathrm{M}_{1}\right)$. The exact location of the wheel load that causes the maximum moment to happen is where the largest tensile strain is calculated, and that will contribute to the initiation or propagation of the new crack length.

As can be seen from Figure 14, rotation is caused at the ends of the slabs A and B and the angles to the horizontal axis created are assumed to be the same as the angles created in the asphalt layer due to the bonding between the two layers. These angles are used in the slope deflection method assuming that the asphalt layer above the debonded length is treated as an independent beam.

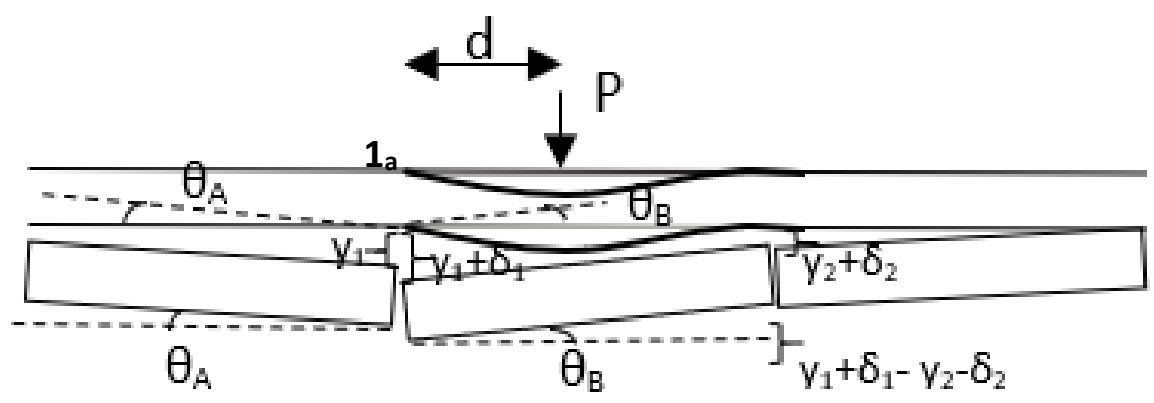

Figure 14: Deflections and angles of slabs. 
In addition to the bending stresses that develop in that area, shear stresses also exist both due to the external load but also due to surface compression forces from the wheel-pavement interaction. At the edge of the wheel load in the contact area between the tyre and the surface of the pavement, an area of high shear is created due to the compressibility of the asphalt surface. According to Thom (2014), each element of the tyre tread would be in compression leading to a Poisson's effect horizontally in the contact area. This would result in a shear stress developing in the surface to eliminate this effect, which can be big enough to cause a crack initiation.

The following flowchart in Figure 15 shows the procedure followed in the top-down cracking model to estimate the total crack length from top-down. The effect of a wheel load at a distance $d$ from the joint between two slabs causes a vertical displacement of the asphalt layer at that point, lowering the level of the asphalt while it takes an S-shape. This also leads to the rotation of the slabs while leading to the asphalt layer creating angles to the original horizontal line. This vertical displacement at that point creates a bending moment and shear forces in the asphalt above the pre-existing joint (or crack) in the underlying layer.

The second effect of the wheel load is a surface shear stress appearing due to the compressibility of the asphalt at the edge of the wheel load. Both these effects lead to the calculation of the maximum tensile strain which is a result of an incremental procedure. In this procedure the distance of the wheel from the joint is initially equal to the radius of the wheel and it increases after every increment to find the location where the maximum tensile strain develops. That location is held as the critical location and that maximum tensile train is used to estimate the crack propagation rate with the help of a fatigue law. In this case as well, the local elastic asphalt modulus of the asphalt due to a crack from the top is reduced and updated for the next iteration. 


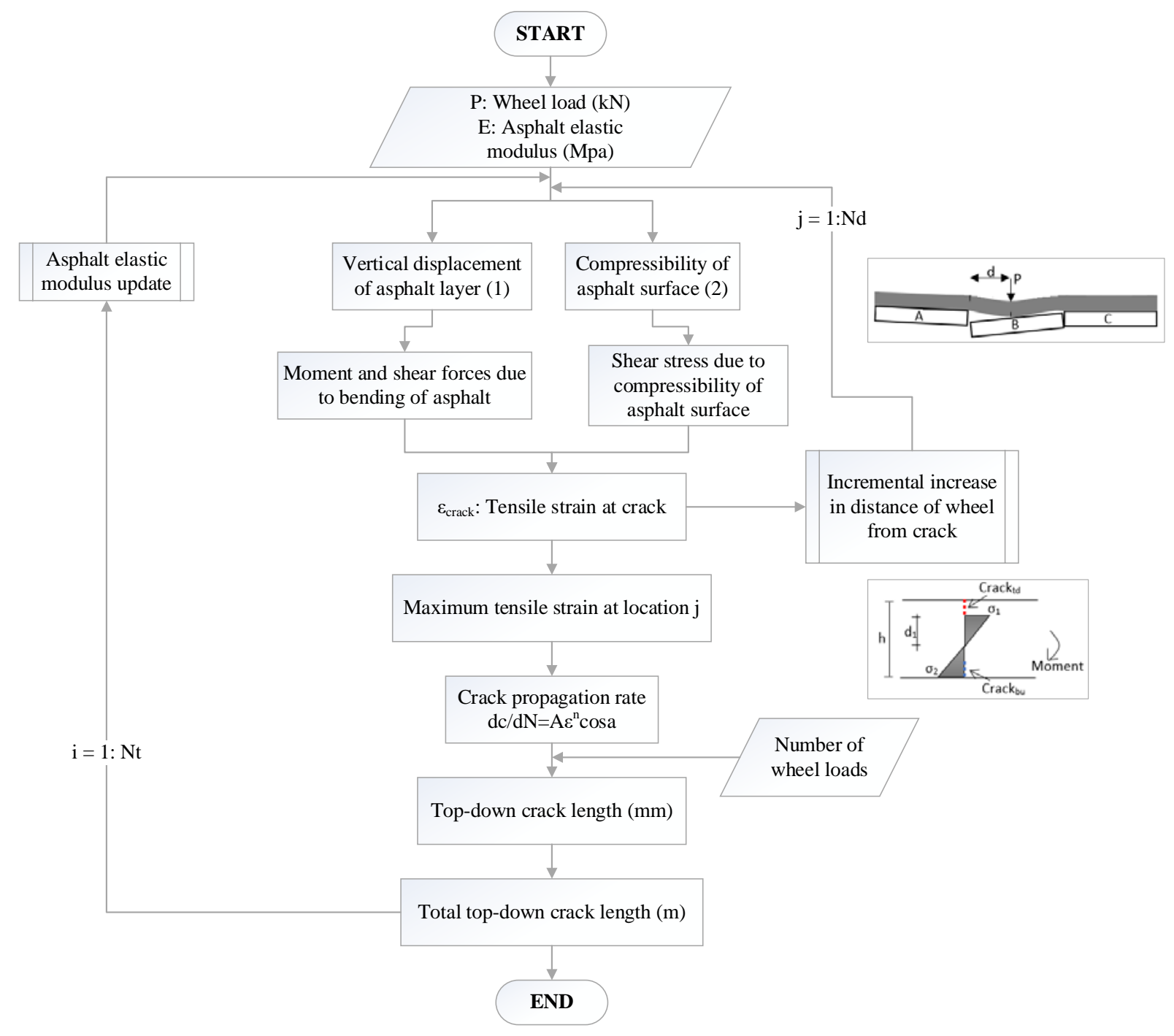

Figure 15: Flow chart for top-down cracking mechanism.

Further information for the top-down cracking mechanism is given regarding:

- the bending strain at the crack,

- the shear strain in the asphalt,

- the tensile strain calculation,

- the crack propagation rate,

- the crack length calculation and,

- the reduction of the asphalt elastic modulus. 


\section{Bending Strain at crack}

The stress distribution through the thickness of the asphalt is shown in Figure 16. The stresses $\sigma_{1}$ and $\sigma_{2}$ can be seen, as well as the length $d_{1}$ which is the distance of the neutral axis of the asphalt beam from the stress $\sigma_{1}$ at the crack tip. When the moment is as shown in the figure, the top part of the beam is in tension and the bottom area is in compression. Even though there is a crack at the bottom area of the beam due to the bottom-up cracking procedure, stress would still be distributed over it due to the compression in that area.

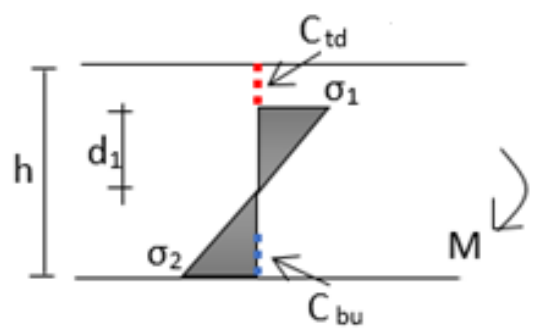

Figure 16: Stress distribution with top and bottom crack.

Due to force and the moment balance of the stresses in the asphalt, and after taking into account the change of location of the neutral axis only when the top down crack is in the area of tension, then the stress at the tip of the crack would be:

$$
\sigma_{1}=\frac{3}{2} \frac{\mathrm{M}}{\mathrm{d}_{1}^{2}}
$$

Thom (1990) showed that $\mathrm{M}$ is given by

$$
\mathrm{M}=\frac{\mathrm{EI}}{\mathrm{d}}\left(\frac{6 \delta_{1}}{\mathrm{~d}}-4 \Sigma \theta_{1}\right)
$$

Where $\delta_{1}$ and $\theta_{1}$ are shown in Figures 11 and 13, $\delta_{1}$ is the differential deflection, slab B less slab A and $\theta_{1}$ is the total angle created at location $1_{\mathrm{a}}$ and $1_{\mathrm{b}}\left(\theta_{1}=\theta_{\alpha}+\theta_{\beta}\right)$. The procedure to estimate them is detailed in (Thom, 1990).

The bending strain at crack would be given by:

$$
\varepsilon_{\text {crack }}=\frac{\sigma_{1}}{E}
$$


When the crack developing from the bottom increases and overcomes the neutral axis of the beam, then that effectively reduces the asphalt thickness which is used to estimate the top-down bending stress in the beam.

\section{Shear strain in the asphalt}

Due to the wheel load on the surface of the asphalt layer, the appearance of a deep region of shear stress is noticeable, which can lead to a tensile strain in a direction at an angle to the horizontal direction (Thom, 2000). Surface shear strain can be attributed to the appearance of asphalt compression and therefore, the prediction of top-down cracking is based on the modelling of the compressibility of the asphalt at the edge of the wheel load (Thom et al., 2002). At the location of maximum moment, the bending shear strain and the surface shear strain are calculated:

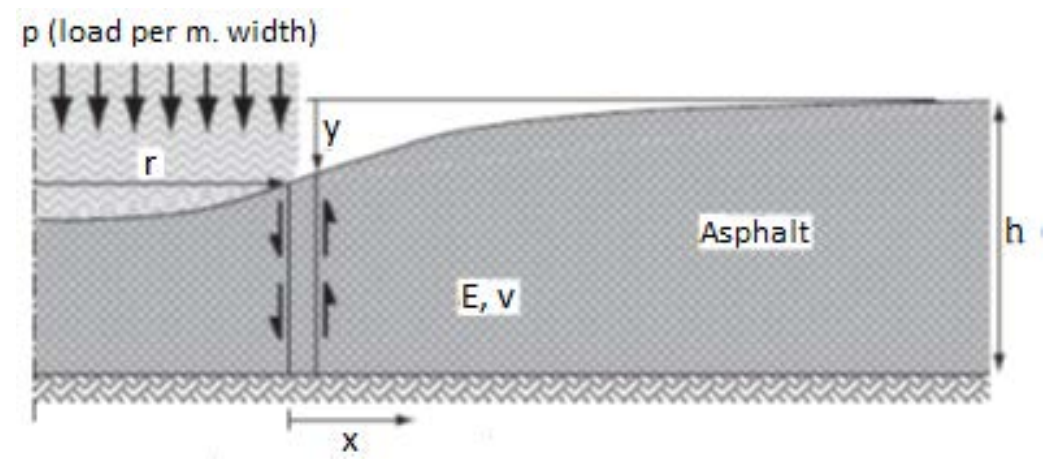

Figure 17: Wheel load and surface profile (Thom, 2000).

Thom (2014) studied top-down cracking and worked out an approximation to estimate surface shear strain due to a wheel load. The suggested equation for modelling the surface profile is given by:

$$
y=\frac{p h}{E} e^{\left[\left(\frac{x}{h}\right) \sqrt{6(1+v)}\right]}
$$

And:

$$
\frac{d y}{d x}=\frac{p}{E} \sqrt{6(1+v)}
$$

Where:

P: Wheel load per m. width $(\mathrm{kN} / \mathrm{m})$

h: Asphalt thickness (m) 
v: Poisson's ratio

E: Asphalt elastic modulus

But it is assumed that the surface shear strain $\left(\gamma_{s}\right)$ at $x=0$ is:

$$
\frac{\mathrm{dy}}{\mathrm{dx}}=\gamma_{\mathrm{s}}
$$

In addition, a component of shear stress is generated by bending of the asphalt layer which is given by and the equivalent bending shear strain $\left(\gamma_{\mathrm{b}}\right)$ is given by:

$$
\gamma_{\mathrm{b}}=\frac{\mathrm{F}}{\mathrm{GH}_{\mathrm{eff}}}
$$

Where:

F: Shear force in asphalt. The detailed procedure for calculating F can be found in Thom (1990).

G: Asphalt shear modulus

$\mathrm{H}_{\text {eff: }}$ Effective asphalt thickness (uncracked thickness)

The total shear strain would be:

$$
\gamma=\gamma_{\mathrm{s}}+\gamma_{\mathrm{b}}
$$

\section{Tensile strain calculation}

Regarding the tensile strain in the region of the crack tip, its calculation is based on Mohr circle of strain calculations (Thom, 2002). This is because of the existence of bending shear stress through the asphalt thickness as well as a surface shear stress in the asphalt. From Mohr circle theory we know generally that:

$$
\varepsilon_{1,2}=\frac{\varepsilon_{\mathrm{x}}+\varepsilon_{\mathrm{y}}}{2} \pm \sqrt{\left(\frac{\varepsilon_{\mathrm{x}}-\varepsilon_{\mathrm{y}}}{2}\right)^{2}+\left(\frac{\gamma_{\mathrm{xy}}}{2}\right)^{2}}
$$

And,

$$
\tan 2 a=\frac{\gamma_{\mathrm{xy}}}{\varepsilon_{\mathrm{x}}-\varepsilon_{\mathrm{y}}}
$$


Assuming that there is no tensile strain in the y direction $\left(\varepsilon_{\mathrm{y}}=0\right)$ and just horizontal strain $\varepsilon_{\mathrm{x}}$, which would be the equivalent to the bending strain at the crack tip ( $\left.\varepsilon_{\text {crack}}\right)$, eq. (2.13) becomes:

$$
\varepsilon_{1,2}=\frac{\varepsilon_{\mathrm{x}}}{2} \pm \sqrt{\left(\frac{\varepsilon_{\mathrm{x}}}{2}\right)^{2}+\left(\frac{\gamma_{\mathrm{xy}}}{2}\right)^{2}}
$$

Where:

$\varepsilon_{x}$ : bending strain at crack

$\gamma_{\mathrm{xy}}$ : shear strain

So, equation (2.20) becomes:

$$
\varepsilon_{\mathrm{t}}=\frac{\varepsilon_{\text {crack }}}{2} \pm \sqrt{\left(\frac{\varepsilon_{\text {crack }}}{2}\right)^{2}+\left(\frac{\gamma}{2}\right)^{2}}
$$

And equation (2.19):

$$
\tan 2 a=\frac{\gamma}{\varepsilon_{\text {crack }}}
$$

\section{Crack propagation rate and crack length calculation}

Progression of the crack through the asphalt layer is based on fatigue law where the crack propagation rate is assumed to be proportional to the power of the strain near the crack tip (Thom, 2014) in a similar way to bottom-up cracking, except in this case the appearance of shear causes the crack to propagate with an angle $\alpha$.

$$
\frac{\mathrm{dc}}{\mathrm{dN}}=\mathrm{A} \varepsilon_{\mathrm{t}}^{\mathrm{n}} \cos a
$$

A, n: constants

The calculation of the length of the crack depth through the thickness of the asphalt follows. That is achieved by multiplying the crack rate with the number of wheel loads that have traversed the pavement section in a selected time increment. It is the same as Eq. (2.8):

$$
\mathrm{C}_{\mathrm{td}}=\frac{\mathrm{dc}}{\mathrm{dN}} \times \mathrm{N}
$$


Where $\frac{\mathrm{dc}}{\mathrm{dN}}$ the crack propagation rate, $\mathrm{N}$ the number of wheel loads and $\mathrm{C}_{\mathrm{td}}$ the length of the crack development from bottom to up.

This crack length refers to a specific time increment (day, week, month). After each time increment the crack length is reinserted in the program and certain parameters are updated until a final crack length for top-down is calculated. For this program that happens when the bottom-up crack meets the top-down crack through the thickness of the asphalt.

\subsection{Reflective cracking model application}

In order to understand the models, two examples follow which show applications of the reflective cracking model due to a static moving load. The first example simulates a composite pavement which was a result of rehabilitation of an existing rigid pavement with a concrete base layer by placing an asphalt overlay. The second example simulates a new flexible composite pavement with a weaker cement bound base. The following table (Table 1) shows the values that describe the properties of the layers and that were input to the program in both examples. From the table it is seen that the stiffness and thickness of the base have different values for each example. The stiffness is lower for the newly constructed flexible composite pavement reflecting the use of lower quality cement bound material.

Table 1: Input for typical composite pavement section.

\begin{tabular}{|l|c|c|}
\hline Pavement property & Rehabilitated & New \\
\hline Asphalt Modulus (MPa) & 4000 & 4000 \\
\hline Asphalt Thickness (m) & 0.1 & 0.1 \\
\hline Base Modulus (MPa) & $\mathbf{2 5 0 0 0}$ & $\mathbf{1 0 0 0 0}$ \\
\hline Base Thickness (m) & $\mathbf{0 . 2}$ & $\mathbf{0 . 1 5}$ \\
\hline Subbase Modulus (MPa) & 200 & 200 \\
\hline Subbase Thickness (m) & 0.25 & 0.25 \\
\hline Subgrade Modulus (MPa) & 100 & 100 \\
\hline Crack Spacing (m) & 3 & 3 \\
\hline Crack Shear Modulus (MN/m ${ }^{3}$ ) & 1000 & 1000 \\
\hline
\end{tabular}

\subsubsection{Example simulation of typical rehabilitated rigid pavement}

The following example regards an existing rigid pavement overlaid with asphalt. The pavement comprises four layers: an asphalt overlay of thickness $100 \mathrm{~mm}$ over an existing concrete pavement of 
thickness 200mm over a granular subbase of thickness of $250 \mathrm{~mm}$ over a subgrade. A cross-section of the pavement can be seen in Figure 18.

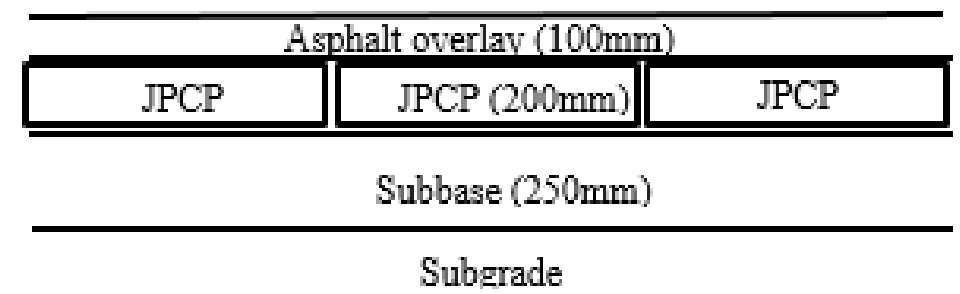

Figure 18: Layers of typical composite pavement section.

Crack growth from the surface and base of the asphalt layer have been simulated and the crack length has been updated after the application of every $3,00040 \mathrm{kN}$ wheel loads and the results are shown in Figure 19 where the progression of the crack from the top of the $100 \mathrm{~mm}$ thick asphalt layer is shown by the dashed line and the progression of the crack from the base of the asphalt layer is shown by the continuous line.

It can be seen from this figure that failure occurs after the application of approximately 4.25 million wheel loads and that the failure mechanism in this case is a crack growing from the surface of the asphalt with cracking from the base of asphalt progressing at a much slower rate due to the thickness and stiffness of the concrete layer.

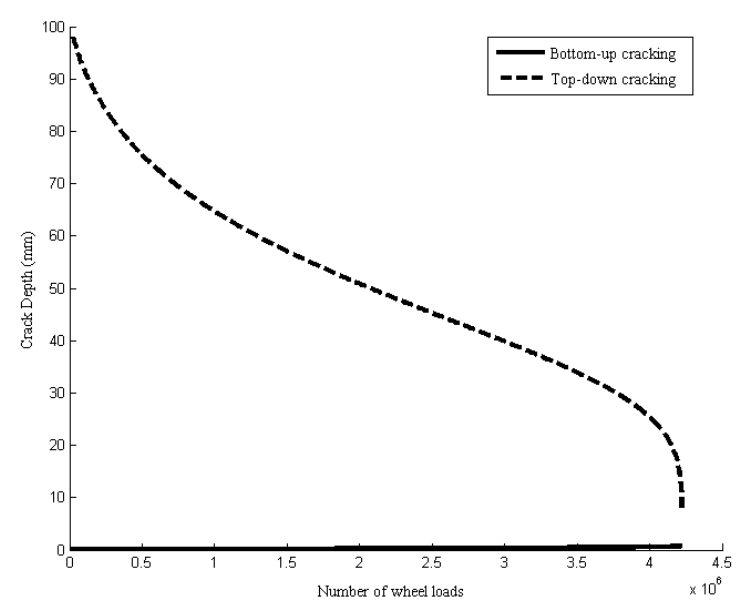

Figure 19: Crack development plot in typical rehabilitated rigid pavement section. 


\subsubsection{Example simulation of typical new flexible composite pavement section}

A weaker cement bound layer was used in the simulation of a new built composite pavement and results are shown in Figure 20. It can be seen from this figure that failure occurs after the application of approximately 2.4 million standard wheel loads and that the failure mechanism in this case is a combination of top-down cracking and bottom-up cracking at similar rates.

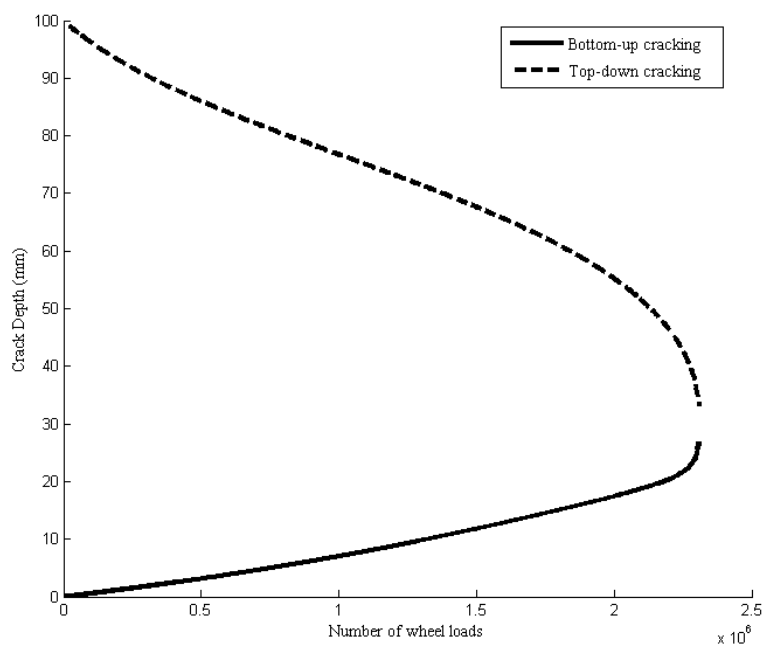

Figure 20: Crack development plot in typical new flexible composite section.

After comparing the two examples it is obvious that in the case of the new flexible composite pavement bottom-up cracking develops in a greater degree than the case of the rehabilitated rigid pavement. That of course can be justified due to the base layer being a cement bound base and not a rigid concrete layer which means its elastic modulus property is lower.

\subsection{Parametric study of traffic driven reflective cracking model}

In this chapter a sensitivity analysis of the parameters in the model is given to identify those with the greatest influence on the result. The key variables in the traffic driven reflective cracking model using a static moving load are the elastic stiffness moduli of the materials (asphalt, concrete, subbase, subgrade), the layer thicknesses, the crack spacing and the crack shear modulus.

The number of wheel loads to failure is used as the criterion to identify the pavement's life. Failure of one location is considered when the reflective crack has propagated through the thickness of the 
asphalt layer and the bottom-up crack meets the top-down crack. This type of failure is used to identify the sensitivity of the results to each parameter.

In order to identify the significance of each parameter, baseline values are selected initially to represent the values of the key parameters. The number of wheel loads to failure which is a result of using the baseline parameter values is found first, and is used as a reference number to normalize the rest of the results. Following, the number of wheel loads to failure are found each time by varying one main parameter at a time, while the rest remain stable. This way their effect on the result can be shown. Assuming that the baseline values have a factor of 1 then the variation of the parameters would be within the range of $0.25-2$ with increments of 0.25 .

The set of baseline values selected are relevant to the composite pavements that are a result of rehabilitation of old rigid pavements, when an asphalt overlay is placed on top of the concrete layer. These values are the same presented and used in the example simulations (see Table 1 "Rehabilitated") and represent the typical values that correspond to composite pavement that is a result of rehabilitation of an old rigid pavement. These can be seen in the following table (Table 2).

Table 2: Baseline values for different parameters.

\begin{tabular}{|l|c|}
\hline \multicolumn{1}{|c|}{ Pavement property } & Rehabilitated \\
\hline Asphalt Modulus (MPa) & 4000 \\
\hline Asphalt Thickness (m) & 0.1 \\
\hline Base Modulus (MPa) & 25000 \\
\hline Base Thickness (m) & 0.2 \\
\hline Subbase Modulus (MPa) & 200 \\
\hline Subbase Thickness (m) & 0.25 \\
\hline Subgrade Modulus (MPa) & 100 \\
\hline Crack Spacing (m) & 3 \\
\hline Crack Shear Modulus (MN/m ${ }^{3}$ ) & 1000 \\
\hline
\end{tabular}

\subsubsection{Asphalt elastic modulus}

The asphalt layer, plays an important role in the structure of the composite pavement. Except its role as an overlay and to protect the underlying layers it also is an essential part of the structure as it takes load and is a bearing layer. Its influence as a material on the pavement's life would be investigated. In the first simulation the asphalt elastic modulus parameter was varied around its base value in the 
following way (Table 3), while the rest of the parameters remained stable at their baseline values (Table 2).

Table 3: Variation of asphalt elastic modulus.

Asphalt

$\begin{array}{lllllllll}\text { Modulus } & 1000 & 2000 & 3000 & 4000 & 5000 & 6000 & 7000 & 8000\end{array}$

(MPa)

\begin{tabular}{|l|l|l|l|l|l|l|l|l|}
\hline Factor & 0.25 & 0.5 & 0.75 & 1 & 1.25 & 1.5 & 1.75 & 2 \\
\hline
\end{tabular}

Each different value of asphalt modulus has a different effect on the result, and a different number of wheel loads to failure is estimated in each case to show the duration of the pavement's life. These are presented in Table 4, where the number of wheel loads to failure is symbolised $\mathrm{N}_{\mathrm{f}}$. The number of wheel loads to failure are normalised by the number of wheel loads to failure calculated using the default values.

Table 4: Variation of asphalt elastic modulus and results.

Asphalt

$\begin{array}{lllllllll}\text { Modulus } & 1000 & 2000 & 3000 & 4000 & 5000 & 6000 & 7000 & 8000\end{array}$

(MPa)

\begin{tabular}{l|c|c|c|c|c|c|c|c|}
\hline Factor & 0.25 & 0.5 & 0.75 & 1 & 1.25 & 1.5 & 1.75 & 2 \\
\cline { 2 - 9 } Nf & 3000 & 186000 & 744000 & 1953000 & 4050000 & 7251000 & 11718000 & 17568000 \\
\hline \multirow{2}{*}{ Nf Norm. } & 0.0015 & 0.0952 & 0.3810 & 1.0000 & 2.0737 & 3.7127 & 6.0000 & 8.9954 \\
\hline
\end{tabular}

In the following figure these results are depicted. Specifically, the normalised asphalt modulus values (x axis) are plotted against the normalised number of wheel loads to failure (y axis). Note that the parameter values of the asphalt modulus have been normalised by their default value which is 4000 MPa. And the number of wheel loads to failure has been normalised by the number of wheel loads to failure (15.357.000) calculated using the default value of $4000 \mathrm{MPa}$. The effect of the varying asphalt modulus on the number of wheel loads to failure is depicted in Figure 21, where the y axis is shown on a logarithmic scale. The blue line represents the pavement life for every asphalt modulus variations. The grey line represents the ratio of the bottom-up over top-down crack at the point when the pavement fails for each factor. This value is greater than 1 when bottom-up cracking is dominant and less than 1 when top-down cracking is dominant. 
It can be seen that the number of wheel loads to failure increases as the asphalt modulus increases. This is quite reasonable because the stiffer the asphalt layer is, the lower the strains developing in the layer, and the greater its life duration, in regards to reflection cracking. In fact, the values on the $\mathrm{x}$ axis reveal that increasing the value of the asphalt elastic modulus from the base value by a factor of 2, results approximately in an increase in pavement life by a factor of 9 . Whereas decreasing the asphalt elastic modulus by half results in a reduction of pavement life by a factor of about 10 . This non-linear effect of the asphalt modulus variation may be a result of the fatigue law, where the tensile strain depends on the asphalt modulus and is varied by a factor power of 1/0.25.

Another reason affecting the non-linearity of the results may be whether bottom-up cracking or topdown cracking is dominant in each case or whether there is a change of mechanism between the different phases. It can be seen that in the first phase of the increase up to the default value top-down cracking is dominant which leads to a longer pavement life with a higher increase rate, but in the next phase, increasing over the default value, bottom-up cracking is dominant leading to an increase in life but with a smaller rate. This change in mechanism from top-down cracking being dominant to topdown is also a reason why the effect of the asphalt modulus is non-linear.

In lower asphalt stiffness values top-down cracking is dominant, whereas in higher stiffness asphalt values bottom-up cracking is dominant.

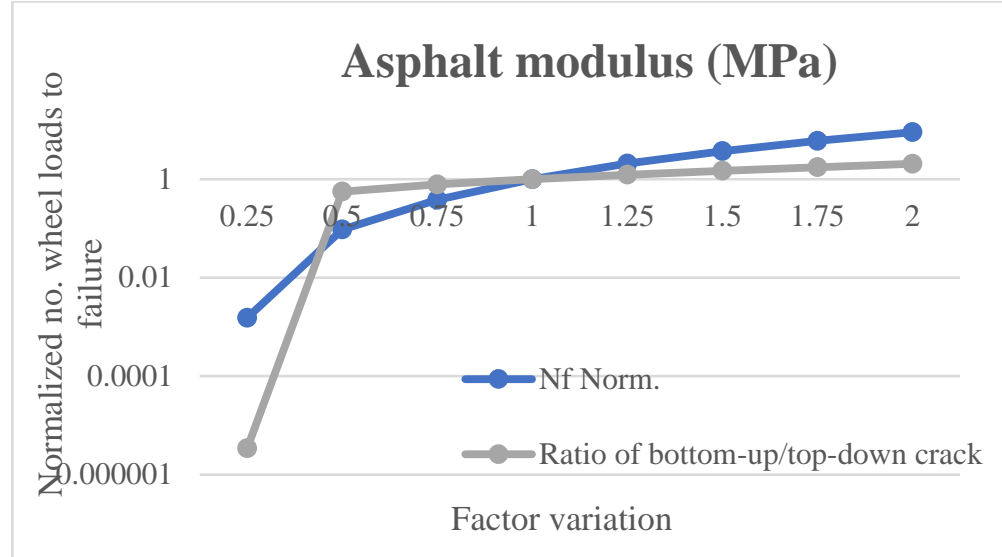

Figure 21: Effect of variation of asphalt modulus to $\mathrm{Nf}$. 


\subsubsection{Asphalt layer thickness}

One of the main methods generally used to delay the appearance of reflective cracks is to increase the asphalt layer thickness. Therefore, the next important parameter that was varied was the asphalt thickness. As can be seen from the results in Table 5 and Figure 22, increasing the asphalt thickness has a positive effect on the pavement's life. Increasing the asphalt thickness by a factor of 2 has as a result the increase of the number of wheel loads to failure also by a factor of around 2.3. This positive correlation is because a greater thickness allows the stresses to dissipate through a greater area of asphalt. Also, there is extra length for the cracks to propagate and eventually meet since we are referring to bottom-up and top-down. The linear effect may be explained due to the fact that after the propagation of cracks in the thickness of the asphalt, the effective asphalt remaining uncracked eventually decreases and the stresses are redistributed over the remaining length, allowing a linear effect on the final result.

In this case it shows that it would be a successful way to increase the pavement's life and delay the appearance of reflective cracks. That is due to the asphalt layer thickness increasing, and the cracks either from the bottom of the asphalt layer or from the top, would have to propagate through a longer distance.

In the lower asphalt thickness values the top-down cracking is dominant and the pavement's life is increased. In the next range of higher asphalt thicknesses however, the bottom-up cracking becomes dominant.

Table 5: Variation of asphalt layer thickness and results.

\section{Asphalt}

$\begin{array}{lllllllll}\text { Thickness } & 0.025 & 0.05 & 0.075 & 0.1 & 0.125 & 0.15 & 0.175 & 0.2\end{array}$

(m)

\begin{tabular}{|l|c|c|c|c|c|c|c|c|}
\hline Factor & 0.25 & 0.5 & 0.75 & 1 & 1.25 & 1.5 & 1.75 & 2 \\
\cline { 2 - 9 } Nf & 231000 & 663000 & 1260000 & 1953000 & 2739000 & 3627000 & 4605000 & 5664000 \\
\cline { 3 - 9 } Nf Norm. & 0.1183 & 0.3395 & 0.6452 & 1.0000 & 1.4025 & 1.8571 & 2.3579 & 2.9002 \\
\hline
\end{tabular}




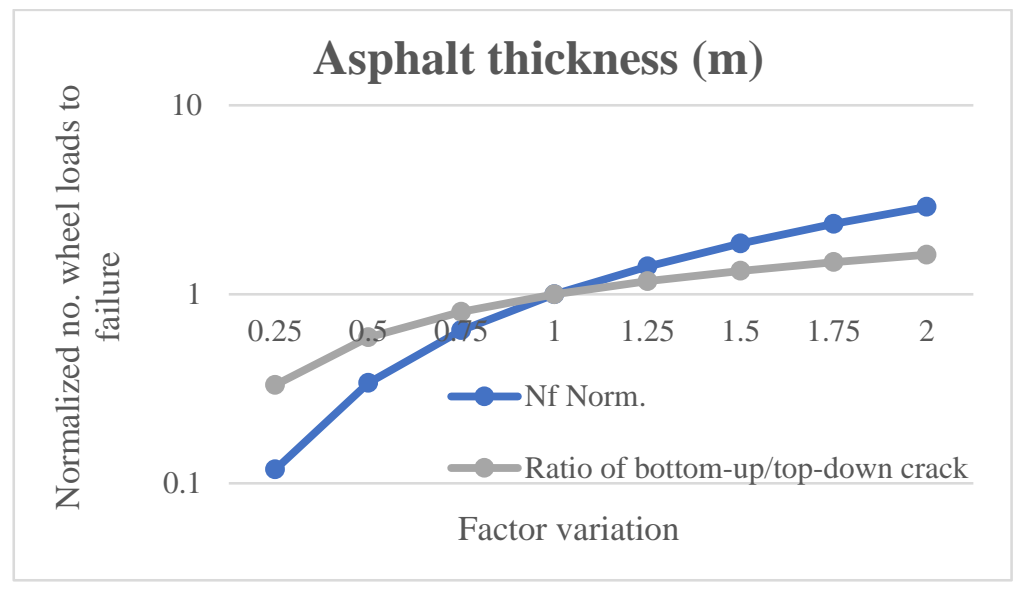

Figure 22: Effect of variation of asphalt layer thickness to Nf.

\subsubsection{Concrete elastic modulus}

Another important layer of the composite pavement structure is the concrete layer that pre-existed from the cracked and jointed rigid layer, which was overlaid with the asphalt layer. The impact of the concrete layer properties on the composite pavement's life is also important.

The result from the concrete elastic modulus parameter variation can be seen in Figure 23. It is seen that when the value of the modulus increases from a factor 0.25 to $0.75,6250 \mathrm{MPa}$ up to $18750 \mathrm{MPa}$ specifically, then the pavement's life also increases. Increasing further the concrete modulus from $18750 \mathrm{MPa}$ to $50000 \mathrm{MPa}$, will have also have a positive effect on the pavements life and the number of wheel loads to failure will increase but with a smaller rate.

Increasing the stiffness of the concrete layer will originally also increase the pavements' life. This is because lower strains are allowed to develop in the asphalt layer and therefore more cycles are needed for the pavement to reach failure. From the results it is seen that when the concrete elastic modulus is in the range of $0.25-0.75$ of the default value then the dominant mechanism is bottom-up cracking, whereas increasing it further would result in the behaviour of the pavement changing and top-down cracking becoming dominant. Therefore, this change in dominant mechanism results in the difference of the concrete stiffness effect on the pavement's life.

This specific design of a pavement regards a composite pavement that is a result of rehabilitation, and therefore, the base layer is usually a concrete layer with a high elastic stiffness. This stiffness is represented in the model in the form of a concrete layer modulus, and the value inserted in the model 
is the initial modulus of the layer when it was uncracked. To take in account of the severity of the cracks the value of the crack shear modulus is used and explained later on.

\begin{tabular}{|c|c|c|c|c|c|c|c|c|}
\hline $\begin{array}{c}\text { Modulus } \\
\text { (MPa) }\end{array}$ & 6250 & 12500 & 18750 & 25000 & 31250 & 37500 & 43750 & 50000 \\
\hline Factor & 0.25 & 0.5 & 0.75 & 1 & 1.25 & 1.5 & 1.75 & 2 \\
\hline$N f$ & 822000 & 1410000 & 1740000 & 1953000 & 2097000 & 2193000 & 2253000 & 2283000 \\
\hline Nf Norm. & 0.4209 & 0.7220 & 0.8909 & 1.0000 & 1.0737 & 1.1229 & 1.1536 & 1.1690 \\
\hline
\end{tabular}

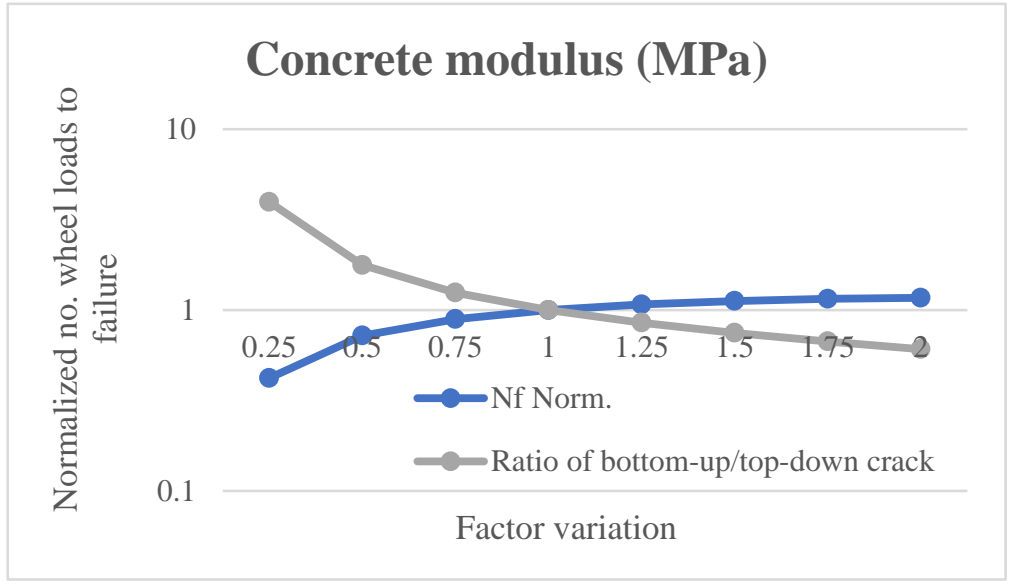

Figure 23: Effect of variation of concrete elastic modulus to Nf.

\subsubsection{Concrete layer thickness}

In regards to the concrete layer thickness, it can be seen from the following results that increasing the thickness has a general result of increasing the number of wheel loads to failure. Increasing from 0 to $0.2 \mathrm{~m}$ has a significant increase in the pavements life, however, increasing the thickness more than $0.2 \mathrm{~m}$ does not have a siginificant difference on the result. The number of wheel loads to failure remain stable having a minimal effect on the pavement's life. The point of changing behaviour may be due to the change of dominant mechanism from bottom-up cracking to top-down. This actually can be confirmed with the grey line, where it is seen that increasing the factor until 0.75 , bottom-up cracking is dominant by far, whereas increasing further the concrete thickness results in bottom-up and topdown cracking being balanced and neither of them being dominant. When this happens, then increasing the concrete layer thickness would not affect the pavement's life. 
Usually, the rehabilitated rigid pavements would have a concrete layer of around 0.2-0.25 m and would not reach thicknesses up to $0.4 \mathrm{~m}$, because of the cost and because of the difficulties that would be created to the load transfer efficiency of the pavement with the use of such thick layers.

\begin{tabular}{|c|c|c|c|c|c|c|c|c|}
\hline $\begin{array}{c}\text { thickness } \\
\text { (m) }\end{array}$ & 0.05 & 0.1 & 0.15 & 0.2 & 0.25 & 0.3 & 0.35 & 0.4 \\
\hline Factor & 0.25 & 0.5 & 0.75 & 1 & 1.25 & 1.5 & 1.75 & 2 \\
\hline Nf & 243000 & 924000 & 1524000 & 1953000 & 2187000 & 2166000 & 2124000 & 2067000 \\
\hline Nf Norm. & 0.1244 & 0.4731 & 0.7803 & 1.0000 & 1.1198 & 1.1091 & 1.0876 & 1.0584 \\
\hline
\end{tabular}

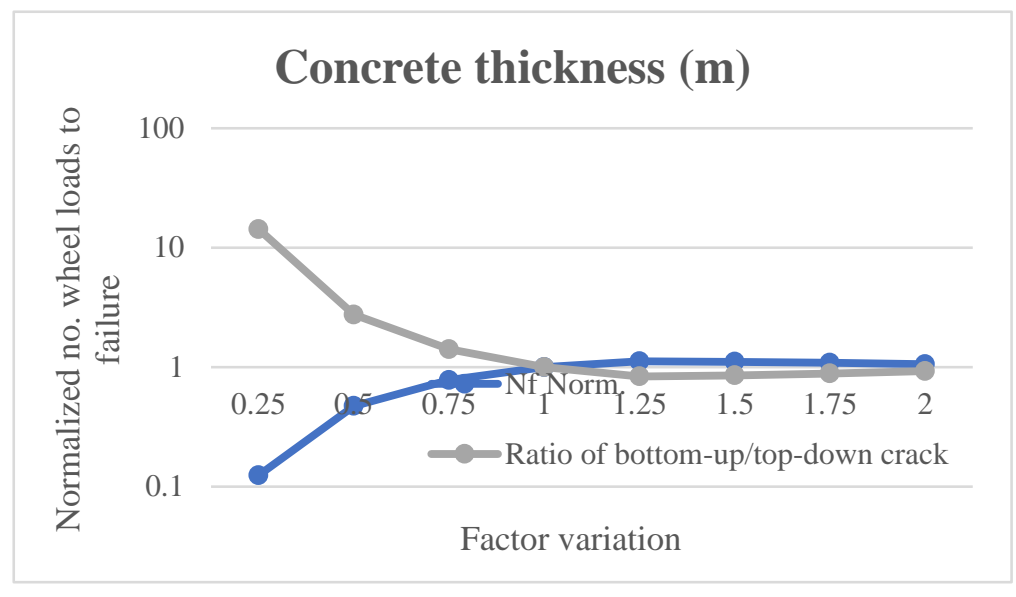

Figure 24: Effect of variation of concrete layer thickness to Nf.

\subsubsection{Subbase elastic modulus}

When increasing the elastic modulus of the subbase, the number of wheel loads to failure increases by a little. An adequately supported pavement has enough stiffness for reduced strains in the pavement. However, increasing the subbase stiffness will not have a big difference on the pavement's life. This is revealed from the results in Figure 25. The dominance of bottom-up and top-down cracking cannot reveal a lot as the values of both are quite close.

Table 8: Variation of subbase elastic modulus and results.

Subbase

\begin{tabular}{|c|c|c|c|c|c|c|c|c|}
$\begin{array}{c}\text { Modulus } \\
\text { (MPa) }\end{array}$ & $\mathbf{5 0}$ & $\mathbf{1 0 0}$ & $\mathbf{1 5 0}$ & $\mathbf{2 0 0}$ & $\mathbf{2 5 0}$ & $\mathbf{3 0 0}$ & $\mathbf{3 5 0}$ & $\mathbf{4 0 0}$ \\
\hline Factor & 0.25 & 0.5 & 0.75 & 1 & 1.25 & 1.5 & 1.75 & 2 \\
\hline
\end{tabular}




\begin{tabular}{l|c|c|c|c|c|c|c|c|} 
Nf & 1191000 & 1644000 & 1836000 & 1953000 & 2037000 & 2103000 & 2157000 & 2202000 \\
\cline { 2 - 9 } Nf Norm. & 0.6098 & 0.8418 & 0.9401 & 1.0000 & 1.0430 & 1.0768 & 1.1045 & 1.1275 \\
\hline
\end{tabular}

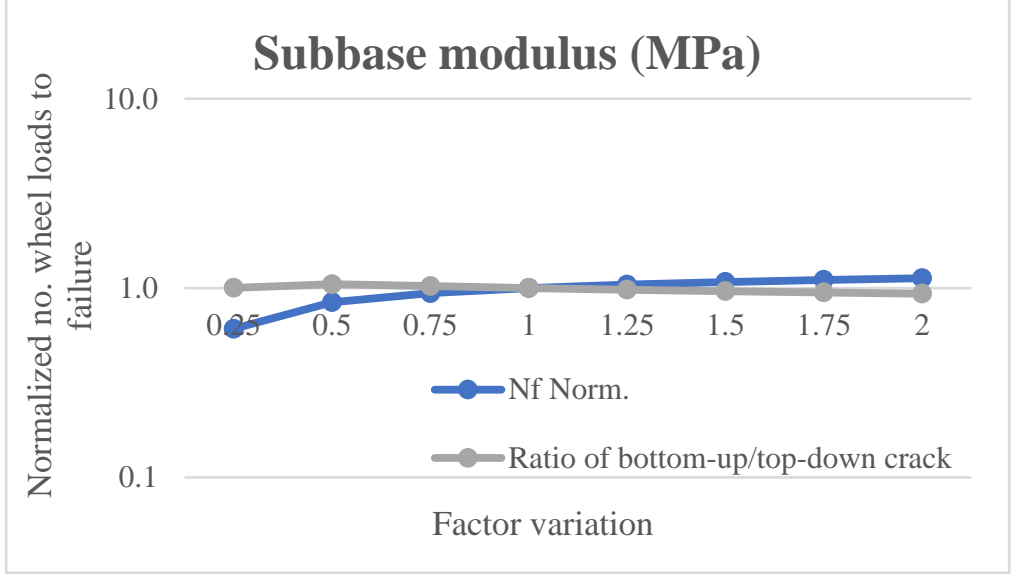

Figure 25: Effect of variation of subbase elastic modulus to $N f$.

\subsubsection{Subbase layer thickness}

The subbase thickness variation showed that increasing the subbase thickness has as a result the increase of the pavements life by a small factor. Significant conclusions cannot be drawn from these results, just that an adequate support of the pavement would have a positive effect on the pavement's life.

It can be said that the values of bottom-up and top-down are quite balanced for all the factor variation and neither is dominant.

Table 9: Variation of subbase layer thickness and results.

Subbase

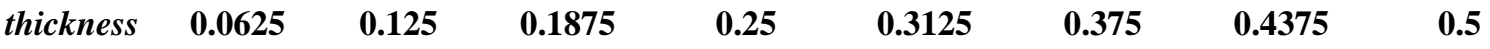

(m)

\begin{tabular}{|l|c|c|c|c|c|c|c|c|}
\hline Factor & 0.25 & 0.5 & 0.75 & 1 & 1.25 & 1.5 & 1.75 & 2 \\
\cline { 2 - 9 } Nf & 1776000 & 1863000 & 1917000 & 1953000 & 1980000 & 1998000 & 2016000 & 2028000 \\
\hline Nf Norm. & 0.9094 & 0.9539 & 0.9816 & 1.0000 & 1.0138 & 1.0230 & 1.0323 & 1.0384 \\
\hline
\end{tabular}




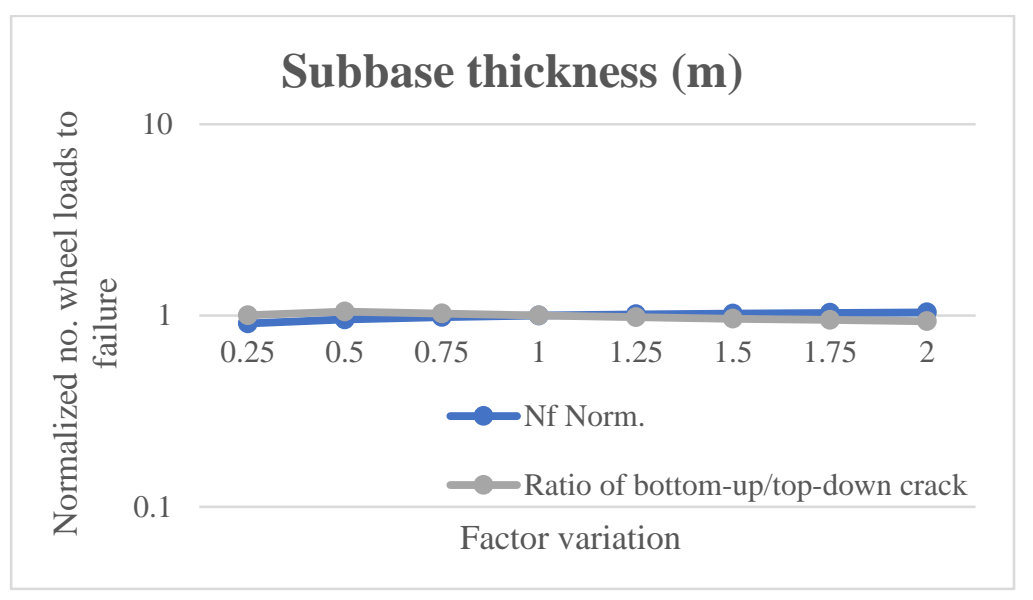

Figure 26: Effect of variation of subbase layer thickness to Nf.

\subsubsection{Subgrade elastic modulus}

The effect of the subgrade modulus can be seen in the following results in Table 10 and Figure 27. It can be seen that increasing the subgrade modulus would have a negative result on the pavement's life by a small factor. This decrease in pavement's life is not significant and therefore no important conclusions can be drawn.

Table 10: Variation of subgrade elastic modulus and results.

\section{Subgrade}

Modulus

25

50

75

100

125

150

175

200

(MPa)

\begin{tabular}{|l|c|c|c|c|c|c|c|c|}
\hline Factor & 0.25 & 0.5 & 0.75 & 1 & 1.25 & 1.5 & 1.75 & 2 \\
\cline { 2 - 9 } Nf & 2025000 & 2028000 & 1998000 & 1953000 & 1893000 & 1818000 & 1734000 & 1638000 \\
\hline Nf Norm. & 1.0369 & 1.0384 & 1.0230 & 1.0000 & 0.9693 & 0.9309 & 0.8879 & 0.8387 \\
\hline
\end{tabular}

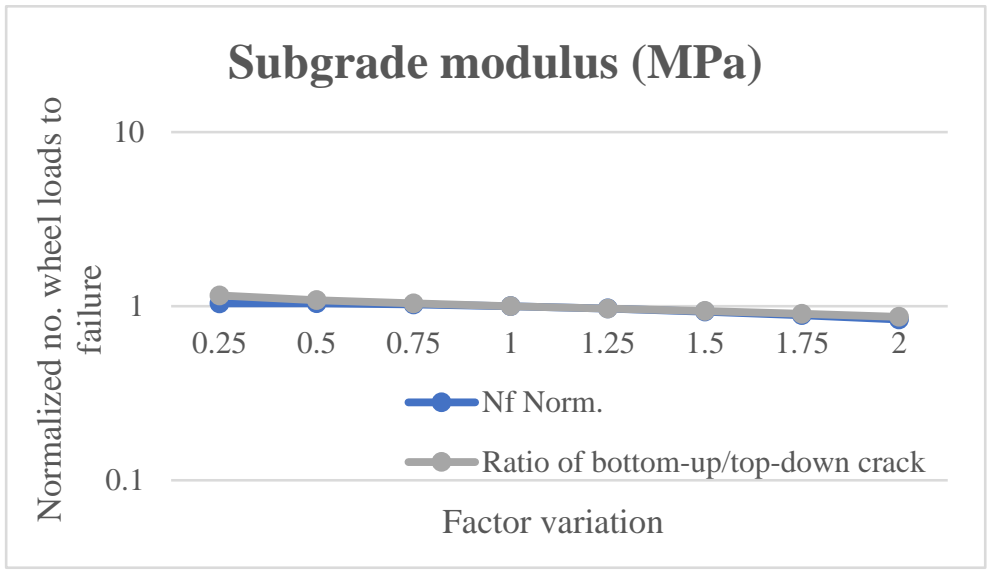

Figure 27: Effect of variation of subgrade elastic modulus to Nf. 


\subsubsection{Crack Spacing}

The crack spacing used in the model represents the distance between the cracks and the joints in the underlying concrete layer. Depending on this crack spacing, the potential areas that can develop reflective cracking can be revealed with the help of distress maps.

The results show that increasing in general the crack spacing would increase the number of wheel loads required for the appearance of a reflective crack. However, increasing it further than $2.25 \mathrm{~m}$ does not cause a significant increase in the pavement's life. What is noticed is that bottom-up cracking is dominant in the first range of values $(0.75 \mathrm{~m}$. - $3 \mathrm{~m}$.) and top-down is dominant in the next range (3m. $-6 \mathrm{~m}$ ) as can be seen by the grey line in Figure 28.

This cannot be explained efficiently, due to the different effective crack lengths that are used in the program for both models, of bottom-up cracking and top-down cracking. The effective crack spacing used is reduced compared to the original one due to the bending that appears also in the slab due to wheel loading.

Table 11: Variation of crack spacing and results.

\section{Crack}

Spacing

0.75

1.5

2.25

3

3.75

4.5

5.25

6

\begin{tabular}{|c|c|c|c|c|c|c|c|c|}
\hline Factor & 0.25 & 0.5 & 0.75 & 1 & 1.25 & 1.5 & 1.75 & 2 \\
\hline$N f$ & 1443000 & 1530000 & 1851000 & 1953000 & 2016000 & 2055000 & 2085000 & 2103000 \\
\hline Nf Norm. & 0.7389 & 0.7834 & 0.9478 & 1.0000 & 1.0323 & 1.0522 & 1.0676 & 1.0768 \\
\hline
\end{tabular}

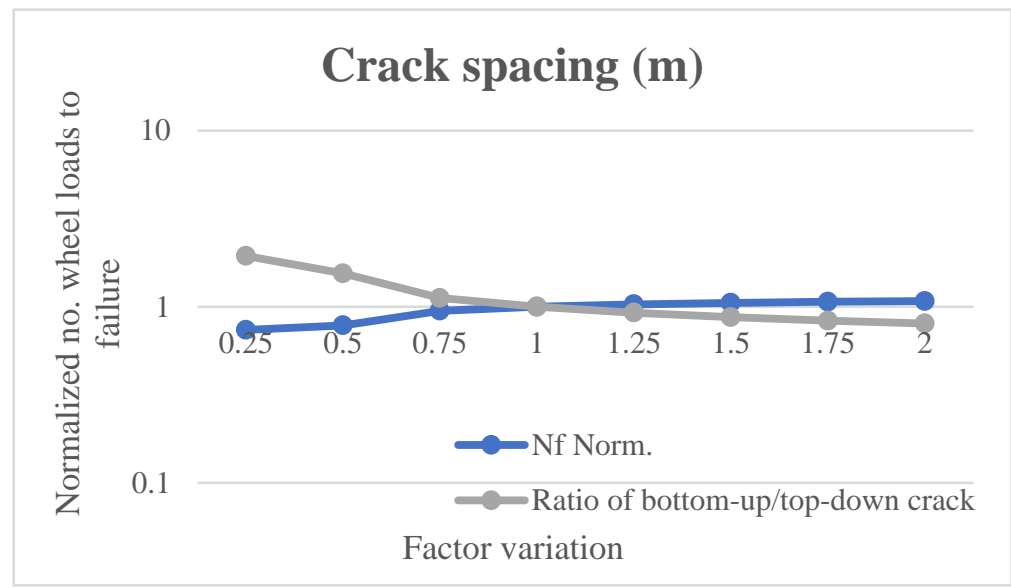

Figure 28: Effect of variation of crack spacing to $\mathrm{Nf}$. 


\subsubsection{Crack Shear Modulus}

The effect of the crack shear modulus is shown separately in Figure 29. It is varied over a wider range (4 orders of magnitude) on a logarithmic scale. A low crack shear modulus can be considered to represent a pavement with wide cracks and low load transfer, whereas a high crack shear modulus can be considered to represent closed cracks with higher load transfer. It can be seen that, as the crack shear modulus increases (and load transfer increases), there is an increase in the number of wheel loads to failure. This positive effect can be seen clearly between the range of 100 and 10000 $\mathrm{MN} / \mathrm{m}^{3}$.

This is an important factor included in the model. It can be used to reveal the state of the underlying cracks before the asphalt overlay was placed. The severity of the cracks can play an important role to the duration which is required before a reflective crack appears.

Table 12: Variation of crack shear modulus and results.

\begin{tabular}{l|c|c|c|c|c|}
\multicolumn{1}{c}{\begin{tabular}{c} 
Crack \\
\multicolumn{1}{c}{$\begin{array}{c}\text { Shear } \\
\text { Modulus }\end{array}$}
\end{tabular}} & $\mathbf{1 0}$ & $\mathbf{1 0 0}$ & $\mathbf{1 0 0 0}$ & $\mathbf{1 0 0 0 0}$ & $\mathbf{1 0 0 0 0 0}$ \\
\hline Factor & 1 & 2 & 3 & 4 & 5 \\
\hline Nf & 459000 & 708000 & 1953000 & 2457000 & 2520000 \\
Nf Norm. & 0.2350 & 0.3625 & 1.0000 & 1.2581 & 1.2903 \\
\hline
\end{tabular}

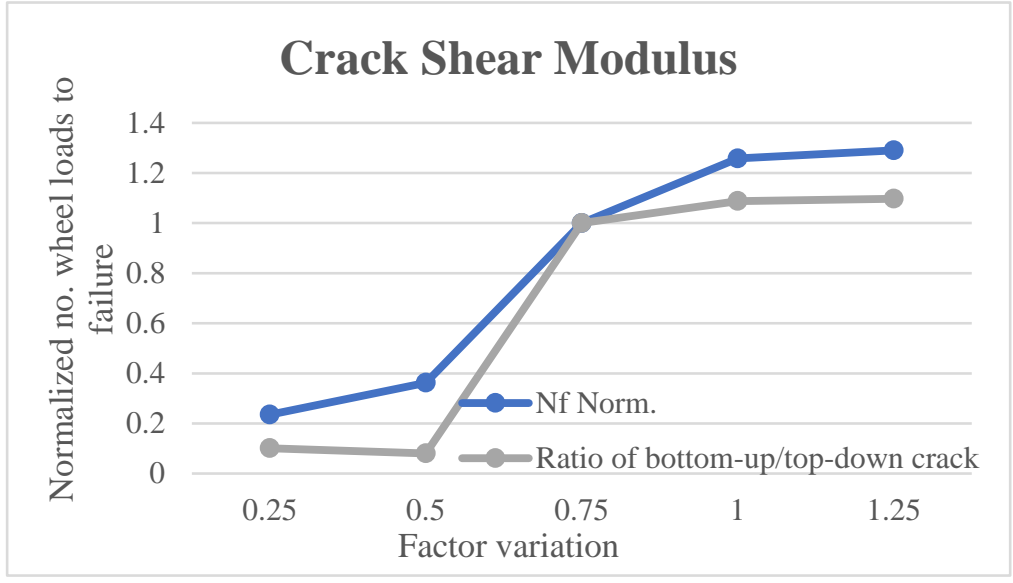

Figure 29: Effect of variation of crack shear modulus to $\mathrm{Nf}$. 


\subsection{Summary}

The goal of this chapter was to present the traffic driven reflective cracking model analyzed in this research study. First of all, previous work in modelling reflective cracking is presented where different methods of accomplishing this are discussed, pointing out that great amount of research has focused on the design of composite pavements, and specifically on the estimation of the appropriate asphalt layer thickness. Other researchers also focused on the prediction of this type of cracking by using finite element analysis or other simplified techniques.

This reflective cracking model consists of two basic mechanisms, the bottom-up cracking and the top-down cracking, accepting the possibility that both directions of cracking can represent reflective cracking. The basic framework of each model is presented with idealized pavement sections and the flowchart of each mechanism. Common between the two is the calculation of a tensile strain in the region of the relevant crack tip, which is used in fatigue law to estimate the crack propagation rate. With this rate after a number of wheel load a new crack length is calculated in either direction.

In order to demonstrate the models, two examples of composite pavements are applied, one representing a new composite pavement and the other an old rehabilitated rigid pavement which was overlaid with asphalt. The outcomes showed that the reflective crack progression and development on the new composite pavement was faster and both directions of cracking had developed, whereas in the other case, there was very little bottom-up cracking development. It was concluded that this was because of the different asphalt elastic modulus values which was much lower for the new pavement in comparison to the old rigid one.

Also, in this chapter the main factors of the reflective cracking model are taken into account to discover the sensitivity of the predictions - number of wheel loads to failure - to the variation of each of these factors. These are the elastic modulus and the thickness of the asphalt layer, of the concrete layer, and of the subbase, as well as the subgrade elastic modulus, the crack spacing and the crack shear modulus. Each parameter was presented separately in this chapter, and in the following figure (Figure 30), the influence of all the parameters on the number of wheel loads to failure is shown. 


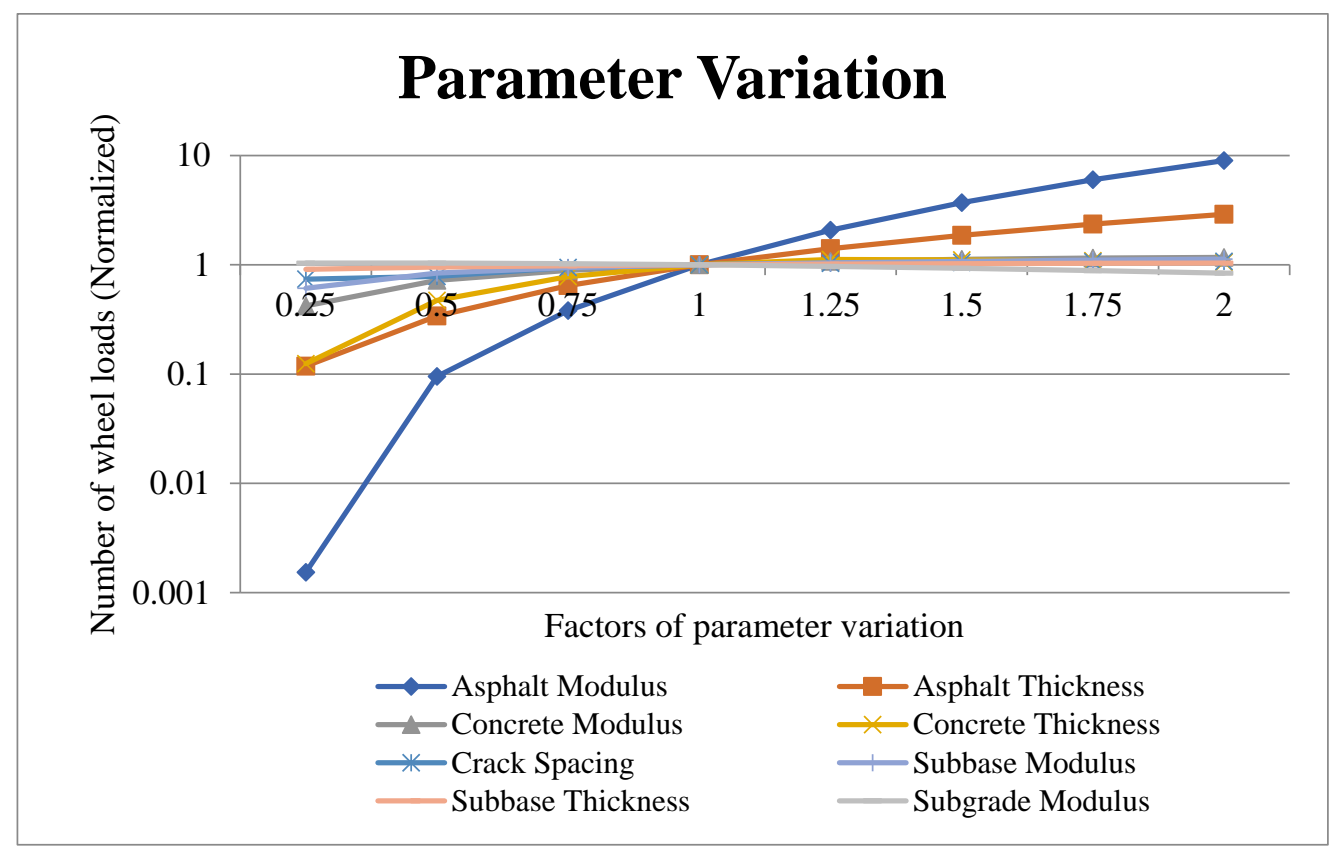

Figure 30: Parameter variation and result on $\mathrm{Nf}$.

The variation of some of the parameters had a positive result on the pavement's life, delaying the appearance of reflective cracking like the asphalt modulus, the asphalt thickness. These two parameters had the strongest influence on the result. Regarding the variation of the concrete elastic modulus an upper limit probably exists, over which the increase of the factor has a lower impact on the result (pavement life increases with a lower rate).

The rest of the parameters such as the subbase thickness or subbase elastic modulus when increased, so is the number of wheel loads to failure but the influence is quite small. On the other hand, the opposite effect has the parameter of subgrade elastic modulus which when increased, has as an effect the reduction of the number of wheel loads to failure.

The results have also been examined in relation to the dominant mechanism in each case, bottom-up cracking or top-down cracking. It can be seen in a few occasions, at the point of change of mechanism from dominant bottom-up cracking to dominant top-down cracking the behavior of the pavement changes and the required number of cycles to failure is decreased.

It has been noticed generally that in the cases where top-down cracking is dominant and there is minimal bottom-up cracking development the top-down crack develops with a higher propagation rate, resulting to quicker failure of the pavement. On the other hand, on the same occasion of dominant 
top-down cracking when the crack originating from bottom is almost balanced with the top-down one then the top-down crack cannot develop with a quicker rate, leading to an increased pavement life. Lastly, when bottom-up cracking is dominant, then pavement life increases as can be seen in the case of parameters like concrete modulus, concrete thickness and crack spacing. 


\section{Thermal Cracking Model}

\subsection{Review of previous work}

The asphalt overlay life depends on various distress modes, and especially on the appearance of cracks. Temperature plays a significant role, first due to the temperature dependent characteristics of the materials of a pavement like for example binder properties (aging, penetration, stiffness etc). Secondly, thermal cycles and low temperatures cause the development of thermal stresses, the accumulation of which result in the increase of the propagation rate of transverse cracks (Shalaby et al. 1996). The complexity of the material properties and the thermal cycles not being easily defined has made the modelling and prediction of thermal reflective cracks difficult.

Reflective cracking, other than traffic induced can also be induced by thermal cycles. These temperature cycles cause the expansion and contraction of the rigid base resulting in the creation of tensile stresses in the asphalt overlay (Trevino, 2004). When these exceed the tensile strength of the asphalt, cracks develop. With the continuous temperature changes these cracks are forced to open and close, causing more tension and therefore damage, depending on the viscosities and thicknesses of the materials (Thom, 2014). These temperature changes can determine in which way the stresses are induced on the overlay. Either restrained shrinkage of the asphalt overlay takes place or crack tip movements cause the development of stresses (Minhoto et al, 2008). There have been some efforts to model reflective cracking due to both traffic and thermal loading.

The mode of reflective cracking from the surface is also explained in a report from Cook and Ellis (2005) regarding reflection cracking in airfields. In general, temperature cycles would cause thermal expansion and contraction of the underlying concrete at the same time as age hardening on the asphalt surface. However, the thickness of the asphalt overlay allows the thermal insulation of the concrete, leading to a greater degree of contraction at the top of the joint. This results in the concrete slabs warping and the greatest tensile strain at that moment being at the surface of the asphalt.

Dave et al. (2007) used finite element analyses with the help of a cohesive fracture modelling approach to simulate reflective cracking. This approach takes into account material response in the area of the crack tip and crack nucleation, initiation and propagation with the help of micro-cracking 
or material softening. A relationship is created between the transfer load capability and the potential opening due to cracking which can offer greater accuracy in modelling reflective cracks. Both thermal and mechanical loads are applied to consider temperature fluctuations and tyre loading from traffic. The critical positions for the tyre loading were considered those immediately above or very close to the pre-existing cracks in the underlying layer of the pavement. The critical condition for the thermal cracking was during cooling cycles, either at a low pavement temperature or with a very high cooling rate (Dave and Buttlar, 2010). Among the results were that the asphalt overlay thickness affects the heat flow in a pavement and therefore the appearance of reflective cracking due to a decrease of temperature. Also, thermal reflective cracking usually appears as bottom-up cracking except if rubblisation of the slabs prior to the overlay has taken place, which will result in top-down cracking. Another approach to model reflective cracking due to both traffic and thermal loading was made by Minhoto et al. (2008). Specifically, in this paper, an effort to estimate the overlay life of the pavement is made, with a finite element analysis model and the calculation of the Von Mises strain due to both types of loading. For the thermal loading the daily pavement temperatures above the pre-existing cracks are input and for traffic loading a $130 \mathrm{kN}$ load is applied. The overlay life is estimated with the help of a fatigue law and it is found that due to both types of loading the overlay life is reduced in winter months compared to the overlay life only due to traffic loading due to the cooling cycles. So, it was concluded that it is important to consider the temperature variation effects in the overlay design against reflective cracking in addition to the effect of traffic loading.

A mechanistic empirical reflection cracking model based on fracture mechanics was developed by Zhou et al (2010) in an effort to take into account both traffic and thermal loading. An equation to calculate the daily crack development based on Paris's law is used, which depends on a combination of bending, shearing and thermal loading effects. The Stress Intensity Factors (SIF) are calculated with the help of a finite element analysis program that considers as influential parameters structure and material factors as well as multilayer bases, and the load distribution. In an effort to use a varied traffic load distribution multiple applications of a single axle load take place instead of a multi-axle traffic loading. Thermal reflective cracking is based on a hybrid low temperature cracking model where thermal stress intensity factors are estimated, and the crack propagation is considered. 
Thom (2014) assumes that a viscous strain develops in the asphalt due to different day and night temperatures, but extreme winter conditions are required for thermally-induced cracking. The rigid layer which consists of slabs of hydraulically bound material, while undergoing thermal cycles will result in the existing cracks or joints to open or close. The asphalt overlay will try to accommodate these movements of the underlying cracks depending on its viscosity and fracture resistance and its thickness. A mechanistic approach rather simplistic is selected to model the pavement during thermal cycles which is able nonetheless to consider factors such as the asphalt's thickness or viscosity and daily thermal cycles. The approach is based on the one-dimensional heat flow equation, taking into account the variation of viscosities of the materials with the temperature changes in discreet time steps. It is considered as a problem of fatigue; therefore, a fatigue equation is used to calculate the number of wheel loads to failure after calibrating the model from experience. In Figure 31 the layers of the composite pavement can be seen as well as tensile stresses due to temperature variation in the asphalt above the area of the joints in the base. The linear viscosity of the asphalt and the shear viscosity of the bond coat between the asphalt and the base are also considered.

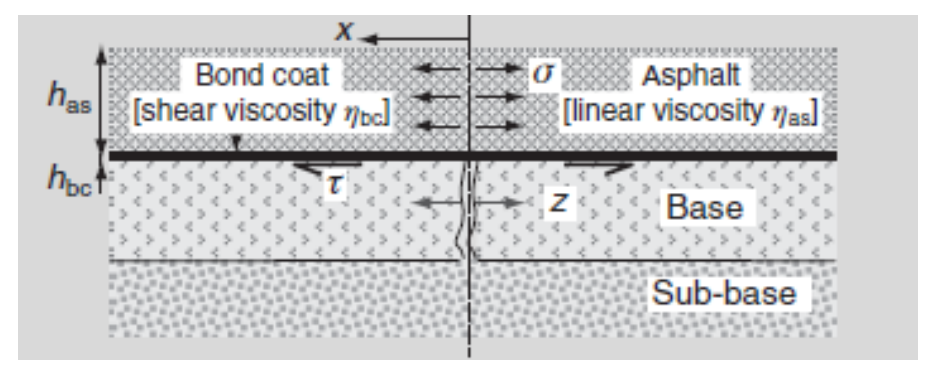

Figure 31: Thermal stresses in composite pavements. (Thom, 2014)

Yin et al. (2007) considers thermal cracking the main factor affecting transverse cracking on asphalt overlaid concrete pavements especially on jointed concrete pavements with long slab lengths. In this research study reflective thermal cracks are not taken into account but only transverse cracks that are created due to thermal loading. When temperature decreases, the asphalt layer goes through contraction and a strain energy develops in the material. An analytical solution is preferred due to the constraints of finite element analysis and a 2D dimensional model is used to account for both the tensile stress along the asphalt overlay as well as the shear stress that develops in the field. Comparing the strain energy with the fracture toughness of the asphalt layer can lead to the prediction of a crack initiation as well as the crack depth. The results seemed good except for the area near the crack tip. 
A transient thermal analysis is used by Shalaby et al. (1996) to model the thermoelastic response of an asphalt overlaid pavement. A displacement formula for special crack tip element of finite element analysis as well as an energy balance approach are considered to take into account the propagation of a thermal crack, with the help of fracture mechanics. Thermal analysis precedes a stress analysis and the appearance of single or multiple thermal cracks are considered. The cracks do not necessarily represent thermal reflective cracks, but transverse cracks on an asphalt overlay that appear due to thermal loading.

\subsection{Thermal cracking model}

Asphalt material is characterized by three specific properties that can describe its thermal behaviour. These are the thermal conductivity, the thermal expansion coefficient and the specific heat capacity. The coefficient of thermal expansion is very important in modelling as it is the value that represents the extent to which the linear dimension of a material changes after a temperature variation of $1^{\circ} \mathrm{C}$. A typical range for hydraulically-bound materials is $0.6-1.4 \times 10^{-5} /{ }^{\circ} \mathrm{C}$ and a typical value for asphalt is around $1.8 \times 10^{-5} /{ }^{\circ} \mathrm{C}$ (Thom, 2014) due to bitumen having a high thermal expansion potential.

The thermal conductivity is also important as it affects the distribution of the temperature with depth through the layers and it represents the efficiency with which heat energy travels through them. It shows the flow of energy created due to a $1 \mathrm{~K}$ temperature difference in a 1 metre length material. Typical value for asphalt or concrete is $2 \mathrm{~W} / \mathrm{mK}$.

The specific heat capacity is another thermal property of materials. It describes the amount of heat energy needed to heat up a specific mass of material by a temperature difference of $1 \mathrm{~K}$. Values typical describing pavement materials are in the range of 800-1000 J/kg K (Thom, 2014).

A thermal cracking model developed by Thom (2014) was adapted and used in this research. The aim of this model is the prediction of the pavement's life expressed as a number of thermal cycles to failure. Repeated sinusoidal temperature cycles are applied on a composite pavement, with the top layer being a single elastic material and the bottom layer a cracked rigid base. These temperature cycles result in expansion and contraction of the layers, leading to the development of cracks due to thermal stresses. 
The daily and hourly temperature variations that take place affect the behaviour of the composite pavements. The difference in material of the two top layers of the pavement leads to a complex development of thermal stresses. The two layers consist of materials that behave in a different way when they undergo temperature changes. The top layer, which consists of bitumen, is normally a viscous material at normal in-service temperatures and has the so-called ability to flow. The bottom layer which is a hydraulic binder is more "brittle" and does not deform easily unless cracks have first been formed.

The temperature difference between night and day, results in either a sudden crack in the asphalt thickness or a crack that will be the result of many cycles of specific strain developments due to daily temperature variations. This model focuses on estimating the hourly temperature variation throughout a day and calculates the strain developed in the asphalt layer after each cycle. A fatigue failure approach is used, and this strain leads to the calculation of the number of temperature cycles to failure.

Different thermal expansion coefficients are attributed to the asphalt layer and the base layer. These define the amount of expansion or contraction that the material develops in linear dimension because of a $1^{\circ} \mathrm{C}$ change in its temperature. Due to the difference in the values of the thermal expansion coefficients for the two materials, the magnitude of expansion (or contraction) differs for the two layers and the gap between the two slabs increases or decreases. As a result, a shear stress field through the thickness of the asphalt layer appears due to the relative movement of the layers.

Due to night and day temperature variation, the expansion and contraction of the rigid base layer results in the opening or closing of the gap between the slabs. This causes the development of a small viscous strain within the top viscous layer of asphalt which is in contact with the base layer. That means that thermally-induced cracking is easier to occur under extreme temperature changes. Also, because of the bitumen's higher value of coefficient of thermal expansion, compared to that of the hydraulic binder's, temperature changes will have a greater effect on its deformation. Significant is also the role of the stiffness of the bitumen, because if we are talking about a soft bitumen it can accommodate the volumetric strains due to temperature variations by adjusting its volume, vertically or laterally, being an incompressible material. On the other hand, if it is a hard material then the 
adjustment of its volume is difficult, and high internal stresses will be induced, which will be able to cause fracture.

\subsection{Model description}

The following flowchart (Figure 32) gives a brief description of the procedure followed in this thermal cracking model. First, after defining the maximum and minimum temperature, the temperature distribution through the pavement layers is estimated at every hour with the help of the heat flow equation. This temperature variation causes heating or cooling of the layers which causes expansion or contraction. This results in development of a strain in the asphalt due to its expansion as well as a viscous strain which is caused by the opening and closing of the rigid base layer gaps between the slabs. The hourly strain contributes to a total thermal strain which is inserted in a fatigue equation and the number of cycles to failure are calculated.

\subsubsection{Temperature distribution}

The first step is to estimate the temperature distribution of the pavement layers and specifically the temperatures at the most important points. As can be seen from the following figure (Figure 33) these are the points of contact between the different layers. They are the top and bottom of the asphalt layer and at the top and bottom of the rigid base layer.

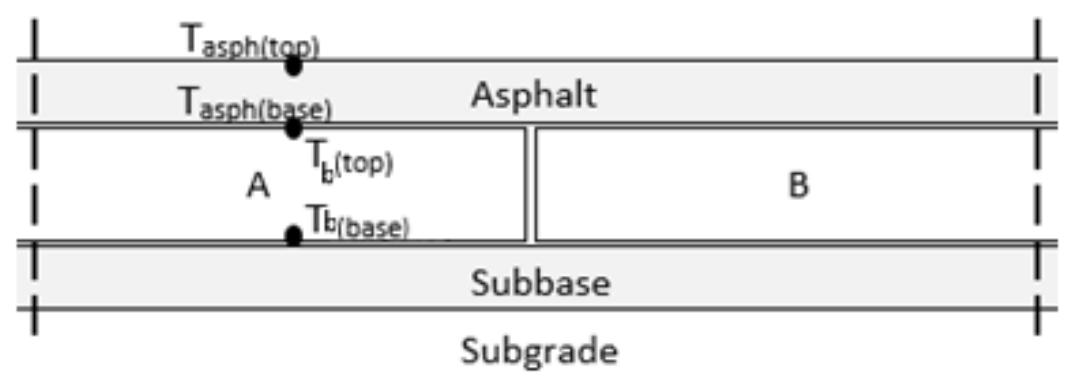

Figure 32: Composite pavement temperatures.

Required for the temperature distribution are the maximum and minimum temperature of each day. The temperature amplitude and the temperature average of that day are calculated and eventually used in a one-dimensional heat equation to estimate the heat distribution through the asphalt layer of the pavement for each hour of the day. 
The following procedure is used to estimate the temperature distribution through the pavement layers. Because of the different temperatures between the asphalt surface and the asphalt base, a heat flow takes place from the asphalt surface through the pavement layers. This can be seen in Figure 34 .

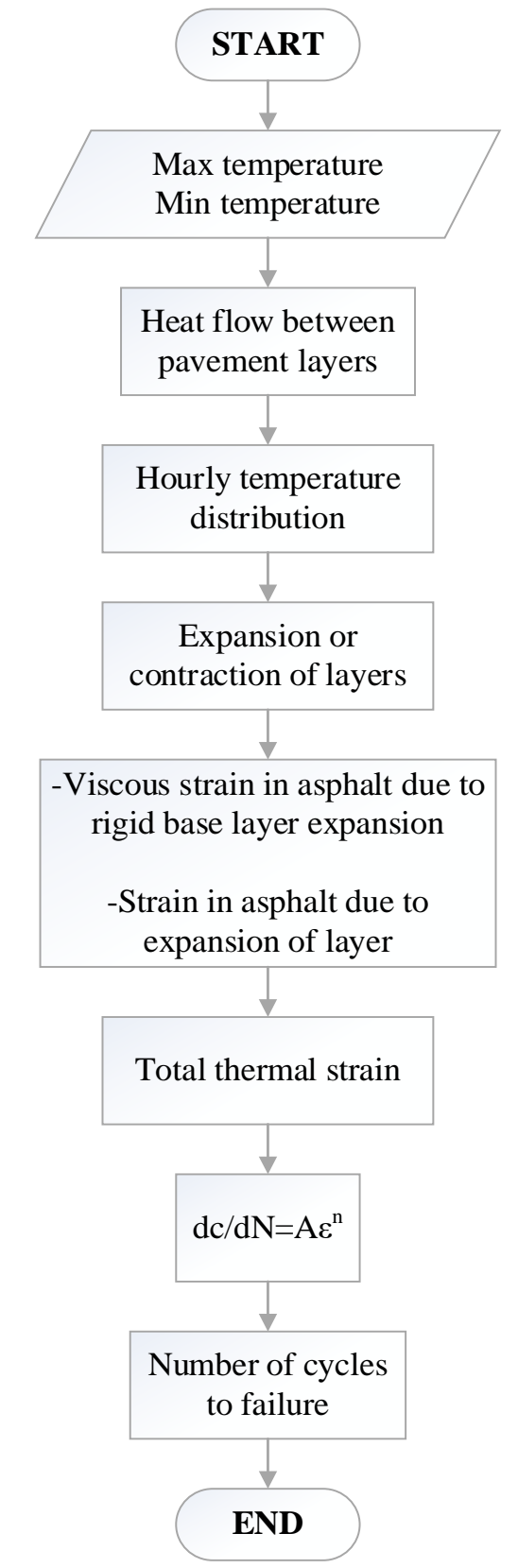

Figure 33: Flow chart of thermal cracking model.

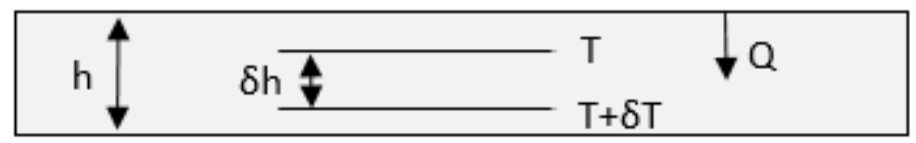


The one-dimensional heat flow would be represented by the following equation:

$$
\mathrm{Q}=\frac{\mathrm{dT}}{\mathrm{dh}} \mathrm{k}
$$

Where:

Q: Heat flow

T: Temperature

$\mathrm{h}$ : Asphalt thickness

k: Thermal conductivity

But from the heat equation:

$$
\frac{\mathrm{dT}}{\mathrm{dt}}=\frac{\mathrm{dQ}}{\mathrm{dh}} \frac{1}{\mathrm{C} \rho}
$$

So, from equations (3.2) and (3.1):

$$
\frac{\mathrm{dT}}{\mathrm{dt}}=\frac{\mathrm{k}}{\mathrm{C} \rho} \frac{\mathrm{d}^{2} \mathrm{~T}}{\mathrm{dh}^{2}}
$$

Solving this differential equation gives the solution with which the temperature distribution of the pavement layers is estimated. The solution to the differential equation (3.3) would be of the form (Thom, 2014):

$$
\mathrm{T}=\mathrm{T}_{\text {mean }}+\mathrm{T}_{\text {amplitude }} \mathrm{e}^{-\mathrm{Ah}} \sin (\mathrm{Ah}+\omega \mathrm{t})
$$

$\mathrm{T}_{\text {mean }}$ : Mean daily temperature $\left({ }^{\circ} \mathrm{C}\right)$

Tamplitude: Amplitude of daily temperature $\left({ }^{\circ} \mathrm{C}\right)$

h: Asphalt thickness (mm)

$\mathrm{A}=\frac{\mathrm{C} \rho \omega}{2 \mathrm{k}}$

$\omega=\frac{2 \pi}{\mathrm{T}}$ where $\mathrm{T}=24 \mathrm{~h}$

C: Specific heat capacity (|J/kgK)

$\rho:$ Density $\left(\mathrm{kg} / \mathrm{m}^{3}\right)$

k: Thermal conductivity (W/mk) 
An example of an hourly temperature distribution of the asphalt base is given in the following figure according to equation 3.4.

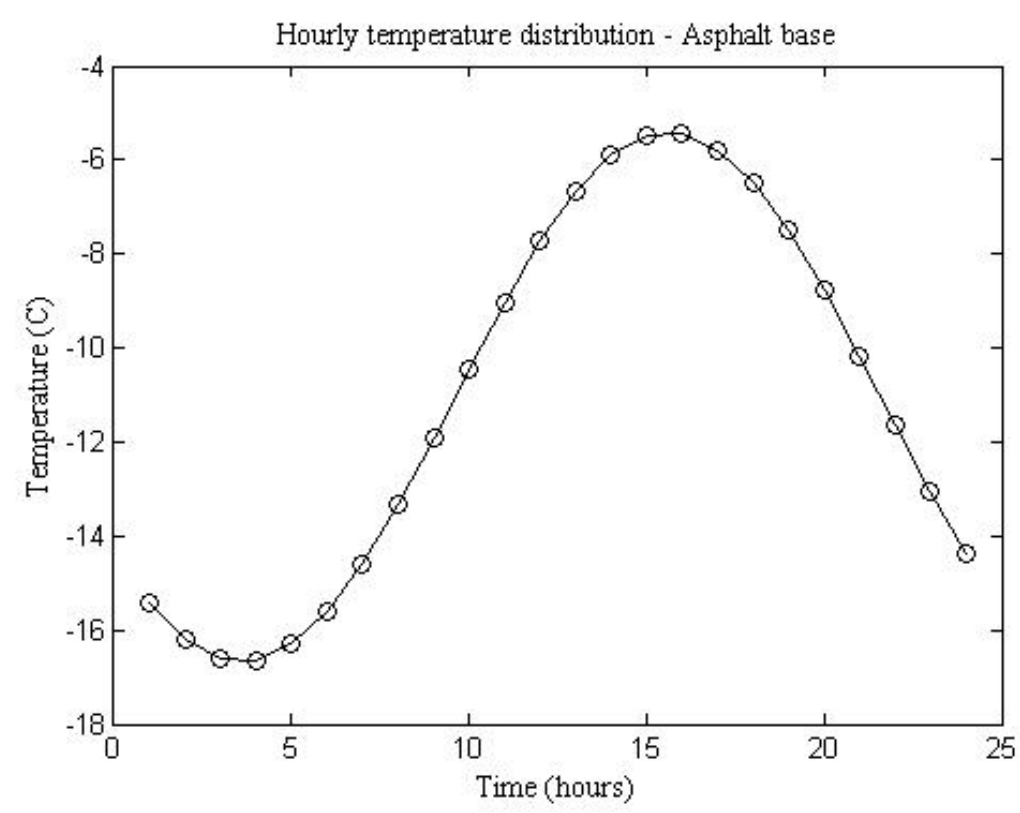

Figure 35: Example of hourly temperature distribution.

It is seen in the above figure that certain factors have been used to accommodate the highest and lowest temperature peak to coincide with the actual hours in a day that that would happen. The highest temperature is noticed at $4.00 \mathrm{pm}$ in the afternoon and the lowest temperature is noticed at $4.00 \mathrm{am}$ in the morning

\subsubsection{Thermal expansion of layers}

The temperature on the top of the cement bound base layer is used to estimate the thermal expansion of the base layer for every hour of the day. The spacing between two pre-existing cracks or joints is required in the process of estimating the amount of expansion developing in the rigid base between the slabs and it is symbolised with the letter L. The way to estimate this expansion is by using the equation:

$$
\Delta \mathrm{x}=\alpha \mathrm{L} \Delta \mathrm{T}
$$

which if applied for the cement bound base layer it would become:

$$
\text { Expansion }_{\mathrm{cbm}}=\mathrm{a}_{\text {base }} \times \mathrm{L} \times \Delta T
$$

Abase: Thermal expansion coefficient of rigid base layer 
This is applied for every hour throughout the day to estimate the maximum gap that occurs, which will induce the maximum strain.

The thermal expansion of the base layer creates a strain which is accommodated in the bottom of the asphalt layer at the point where the asphalt layer is connected to the cement bound base layer, due to the viscosity of the asphalt and its ability to "flow". This strain that the asphalt layer receives from its connection to the base, as well as the strain from the asphalt layer's thermal expansion is the total strain which will be taken into account in a fatigue law equation (Thom, 1990).

In Figure 36, an idealized simulation of a composite pavement model is given. The two layers of the composite pavement are shown, along with the important locations (1 and 2) that are used in the thermal strain calculation procedure. The top layer in the figure represents the asphalt layer with asph representing the thermal expansion coefficient of the asphalt. The bottom layer represents the rigid base layer that consists of slabs A and B with abase representing the thermal expansion coefficient of the cement bound base layer. In order to explain the thermal stress development, the movement rate of points 1 and 2 are examined.

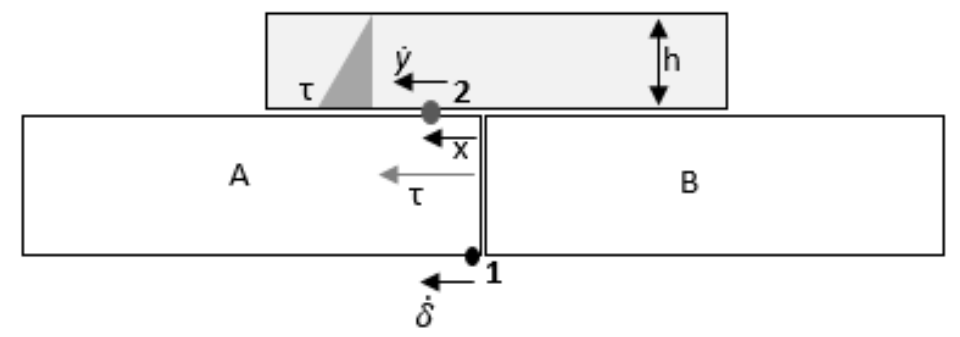

Figure 36: Movement rates between asphalt and CBM layer.

Because of the two movement rates, shear stress will develop in the asphalt layer, and its distribution is shown in Figure 36 with the symbol $\tau$ at the point of contact of the asphalt and the rigid layer.

According to Thom (1990), between the asphalt layer and the rigid base due to the relative movement of the layers a length over which the two layers lose their bonding is considered. That is assumed to be the debonded length, symbolised $d$. The appearance of the debonded length, $d$, affects the relative movement between the two selected points, 1 and 2. The final equation used for the thermal strain rate taking in account the debonded length would be: 


$$
\dot{\varepsilon}=\frac{\dot{\delta}}{\sqrt{\frac{\eta_{t c}}{\eta_{\text {mix }} h t}}+d}
$$

Where $\dot{\delta}$ : movement rate of point of slab 1

$\eta_{\mathrm{tc}}$ : viscosity of tack coat

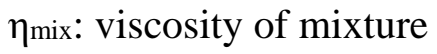

h: asphalt thickness

t: tack coat thickness

d: debonded length between asphalt and rigid base

The procedure to find the strain rate of equation is shown in Thom (1990). This strain rate represents the viscous strain rate that develops due to the relative movement rate of the two layers taking also into account the debonded length. It takes in account the viscosity of the asphalt and of the tack coat between the asphalt and the rigid base. The procedure for the estimation of the viscosities of the asphalt and the tack coat is based on Thom (1990).

\subsubsection{Total strain}

The total strain, that would be used in the fatigue equation, after each temperature cycle would depend on two components: on the total viscous strain from that cycle and on the strain from the asphalt's expansion.

$$
\varepsilon=\text { Total Strain }=\text { Viscous strain }+ \text { Asphalt thermal strain }
$$

Due to the change of the viscous strain rate each hour the average between two consecutive hours is used to estimate the viscous strain of the next cycle.

And the strain due to the asphalt expansion during every hour would be:

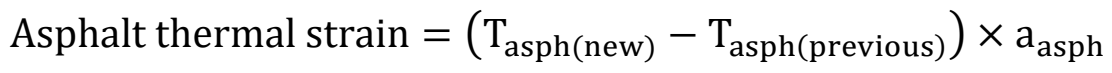

aasph: Thermal expansion coefficient for asphalt layer

$\mathrm{T}_{\text {asph(new): }}$ Temperature at the base of the asphalt of current hour

$\mathrm{T}_{\text {asph(previous): }}$ Temperature at the base of the asphalt of previous hour 
After 24 hours (1 day), the total strain from each hour of temperature change is calculated. The maximum strain that develops through that day is the one used in the fatigue approach to estimate the number of cycles to failure.

\subsubsection{Crack growth and number of cycles to failure}

A fatigue law equation is used which is related to the strain:

$$
\frac{\mathrm{dc}}{\mathrm{dN}}=A \varepsilon_{\mathrm{t}}^{\mathrm{n}}
$$

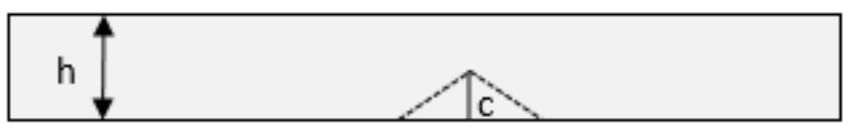

Figure 37: Crack growth in asphalt.

\section{Calibration}

This model has been calibrated against known behaviour of a composite pavement under daily temperature variations. According to the calibration the number of temperature cycles that are calculated from this model are equivalent to $\frac{N}{26}$ number of years till the pavement fails due to thermal loads (see (Thom, 1990) for further details).

\subsection{ThermCrack applications}

In order to apply this model, according to the needs of this research, certain input data was required. The following is the input required and the origin of the data.

- Minimum and maximum daily temperature (LTPP)

- Bitumen and tack coat penetration (Assumption)

- Crack spacing of rigid base (LTPP)

- Thermal expansion coefficient of rigid base (LTPP)

- Thermal expansion coefficient of asphalt (Assumption)

- Debonded length (Mechanical model)

- Thickness of rigid base, asphalt layer and tack coat (LTPP)

- Thermal Conductivity (Assumption)

- Specific Heat Capacity (Assumption)

- Density (Assumption) 
- $\quad$ et $(\mathrm{N}=0)$ (Assumption)

- fatigue slope (Assumption)

- $\quad$ specimen diameter (Assumption)

Initially the goal of the program was to estimate the maximum thermal strain developing. This with the help of fatigue law leads to the number of thermal cycles to failure. The number of thermal cycles to failure after each day, can be used with the help of Miner's law to give the total damage. When the value of the total damage reaches 1 the pavement is considered to have failed in regards to thermal cracking.

\section{Thermal cracking model limitations}

This model is used under certain limitations. First of all, there is no seasonal variation in the way the model works. The only data taken in account are the daily temperatures therefore that is how winter or summer are taken in account. The pavement sections used as validation, due to the availability, belong to climate zones in the following categories: wet/no-freeze, wet-freeze, with no pavement sections belonging to the dry zone. The other limitation is that certain required input data are based on assumptions as is mentioned above and not on the real equivalent data (i.e. bitumen penetration, thermal expansion coefficient of asphalt).

\subsection{Parametric analysis of thermal cracking model}

With the purpose of understanding the way the thermal crack model works, it was important to conduct a parametric analysis which will identify the importance of each factor in the model. The factors selected for this analysis were: the asphalt thickness, the thermal expansion coefficient of the asphalt and of the rigid base layer, the crack spacing (distance between each crack or joint in the underlying layer), and the bitumen penetration value.

These factors were all varied from a specific base value, increasing by factors 1.5 to 4 . The effect on the number of temperature cycles to failure and on the maximum thermal strain developing in the asphalt were investigated. The results are shown in the following figures.

The effect of the various parameters on the strain developing in the thermal cracking situation is shown in Figure 38. It is seen that the greatest effect on the strain have the thermal expansion 
coefficient of the rigid base layer as well as the length of the slabs (spacing between joints). They have the same exact effect on the result due to the equation of expansion that is used in the model $\left(\Delta \mathrm{x}=\mathrm{a}_{\text {base }} \mathrm{L} \Delta \mathrm{T}\right)$. Increasing one of these two parameters by a factor of 4 from the base value will result in an increase of the strain by a factor of 2.5. Important is also the effect from the asphalt thickness parameter. While increasing its value by 4 times, the strain developing decreases by around 0.5 times. It is normal that increasing the asphalt thickness would lead to less strains because that would mean that the stress would be distributed in a wider area in the asphalt layer, therefore causing less damage over each cycle.

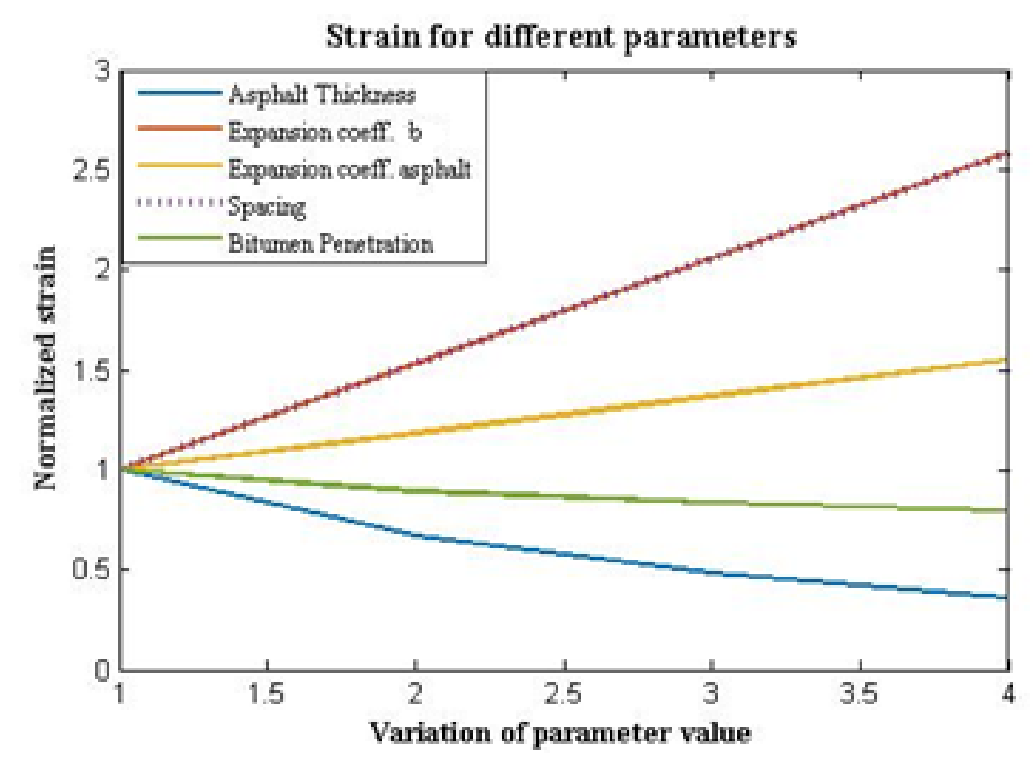

Figure 38: Normalized thermal strain for different parameters.

In the following figure (Figure 39), the effect of all parameters on the number of temperature cycles to failure is shown. Due to the results, specifically the great dependence on one parameter, the asphalt thickness, only the influence of that factor can be seen clearly. With a variation of the parameter's value by a factor of 2 , the number of the temperature cycles to failure increases by around 20 times, and even worse by increasing the parameter's value by a factor of 3 , the resulting number of cycles increases by 50 times. 


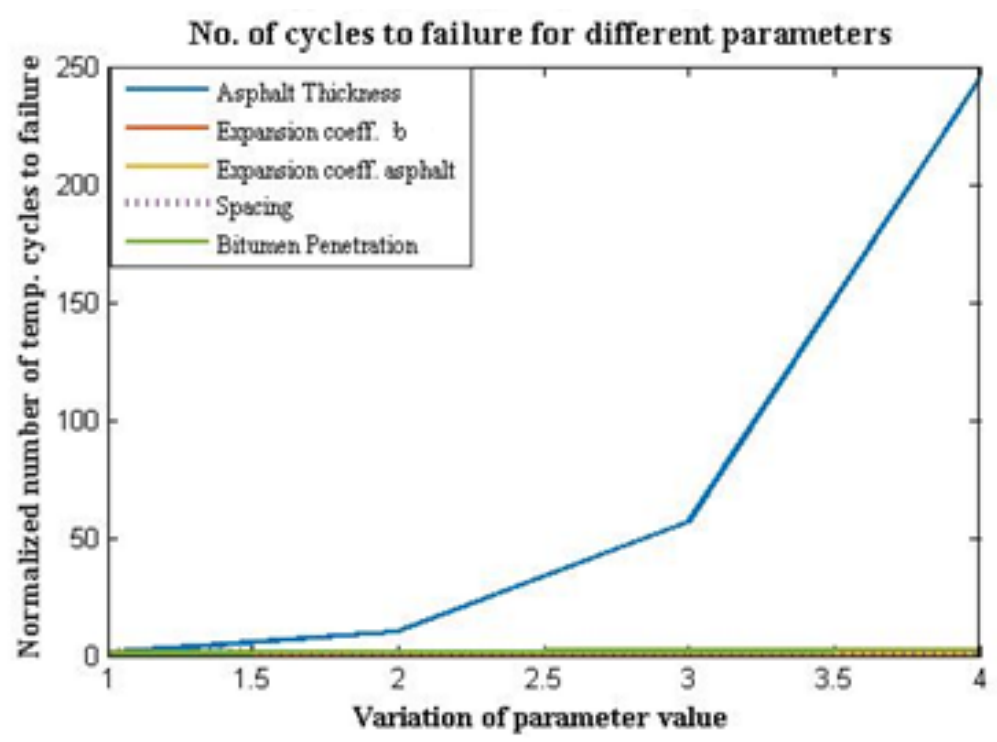

Figure 39: Normalized no. of thermal cycles to failure for different parameters (a).

In order to look at the effect of the rest of the factors on the number of thermal cycles, the asphalt thickness line is removed from the figure and the rest of the parameter lines are left to be seen more clearly in Figure 40. It can be seen that by increasing the bitumen penetration value the pavement's life in terms of thermal fatigue failure increases, whereas by increasing the thermal expansion coefficient of the cement bound base and that of the asphalt, the pavement's life decreases. The results for the coefficient of thermal expansion for the base layer are identical with the results from the effect of the crack spacing.

Also, the effect of the thermal expansion coefficient is worse for the relevant base layer value compared to that for the asphalt, meaning that an increase of this coefficient will create a greater expansion (or contraction) and the strains that the asphalt would have to accommodate would be worse, leading to the decrease in the pavements life. 


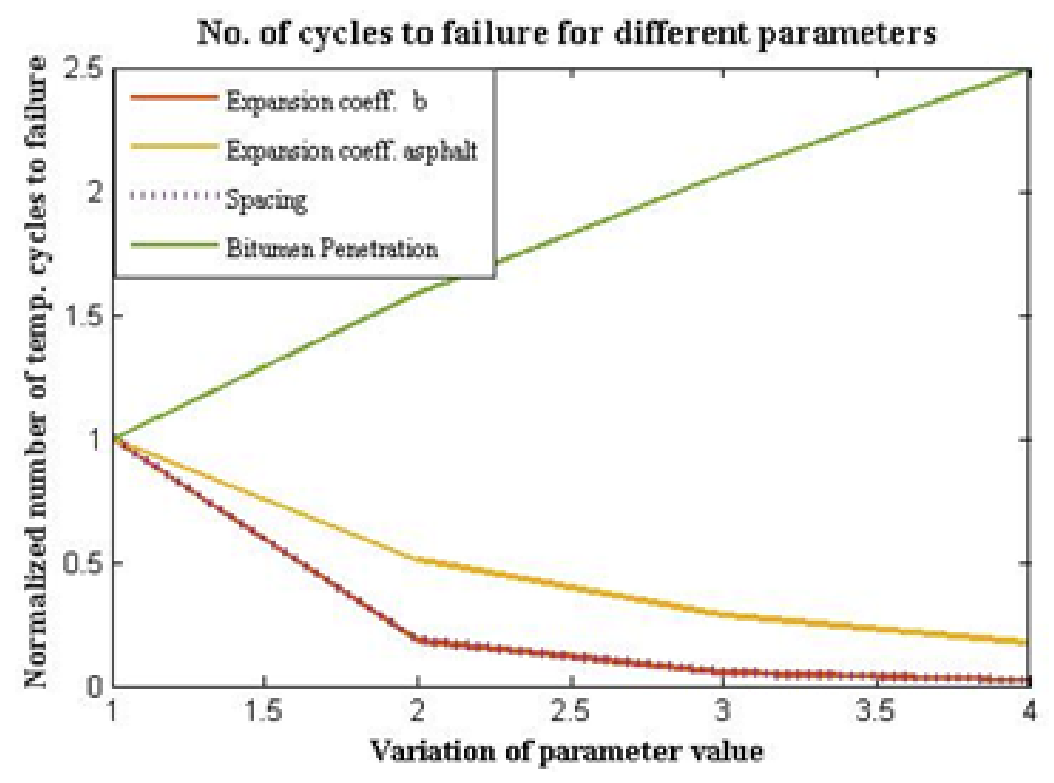

Figure 40: Normalized no. of thermal cycles to failure for different parameters (b).

\subsection{Summary}

In this chapter the thermal cracking model used in this study to predict reflective cracks due to thermal stresses is presented. This model was selected to investigate the effect of temperature cycles on the development of reflective cracks on composite pavements. The mechanisms behind the thermal cracking model are complex but an attempt is made to describe them generally. The interface of the asphalt overlay with the more rigid cement bound base is examined, taking into account the different thermal expansion coefficient of the two layers, which would lead to a different expansion of the layers for the same temperature difference. The viscoelasticity of the asphalt layer allows it to accommodate any changes that occur to the concrete layer with which it is in contact.

A daily sinusoidal temperature distribution is found and along with the different layer interfaces and mechanisms the maximum thermal strain in the pavement is estimated for each day. This is used in a fatigue failure approach to calculate the number of thermal cycles to failure which is the output of the model. The model has been calibrated against known behaviour and the number of cycles is converted to the amount of time needed until the asphalt layer has presented a crack through the whole thickness.

The worst case would be when super-imposing the effects of thermal-induced stresses at the same time as induced stresses due to wheel loading. 


\section{Dynamic vehicle model}

\subsection{Background}

\subsubsection{Vehicle loading \& Pavement performance}

During the early design of roads in the first half of the $19^{\text {th }}$ century, no concise and reliable method existed to account for the influence of vehicles on pavement design. This lead to pavements being designed as typical civil engineering structures which would mean that they would be designed by a maximum load they can resist without damage (Cebon, 1999).

In the early 1960's, the American Association of State Highway Officials (AASHO) Road Test took place which was one of the largest investments and experiments in the investigation of the performance of highway pavement structures. According to the AASHO report (1962), serviceability is a term that was defined as 'the level of a particular pavement's ability to serve the traveling public'. However, 'the trend of serviceability with time or load applications' is what is defined as the pavement's performance'.

An effort was made to predict pavement performance with reasonable accuracy from measurement of deflections, strains and stresses during a critical time period or during construction of the pavement. In their effort to discover a way to measure pavement damage, the concept of the Equivalent Single Axle Loads (ESALs) was conceived (Gillespie, 1993). According to that, damage caused by a number of different wheel loads is converted to damage from an equivalent number of wheel loads that create a known damage. The reference axle load was $18,000 \mathrm{lbs}(80 \mathrm{kN})$ and refers to a single axle with dual tyres. What is clearly explained in Gillespie, 1993, is that ESALs are a "relative" type of pavement, and "one ESAL on a strong pavement corresponds to a much lower proportion of its fatigue life than one ESAL on a weak pavement". This however does not take into account important factors like the roughness of a pavement and any other irregularities.

A very important discovery for pavement design was the use of the 'fourth power law'. It was found that, although there was significant scatter in the data, the fourth power static load of a heavy vehicle axle could be related to the deterioration of a 'pavement's serviceability'. To measure the serviceability of a pavement the concept of 'Present Serviceability Index' was defined with values in 
the range of 0 to 5 , and 5 being having a perfect state of serviceability. So, the 'fourth power law', entails converting regular mixed traffic to a number of Equivalent Standard Axle Loads (ESALs) by applying the following to each axle:

$$
N=\left(\frac{P}{P_{0}}\right)^{n}, \quad n=4
$$

Where $\mathrm{P}_{0}$ is normally $80 \mathrm{kN}$.

This damage exponent may take different values depending on the type of pavement. It has been found that for composite and rigid pavement n can be 8-12 or 11-13 (Cebon, 1999). The choice of the exponent value is very important because of its increasing impact on the number of Equivalent Standard Axle Loads. This can also be seen in the following figure taken from Cebon, where a different exponent each time results in a different number of ESALs. This discovery was a significant milestone in pavement design as it simplified calculations for this process and created a common base of comparison for all pavement management agencies.

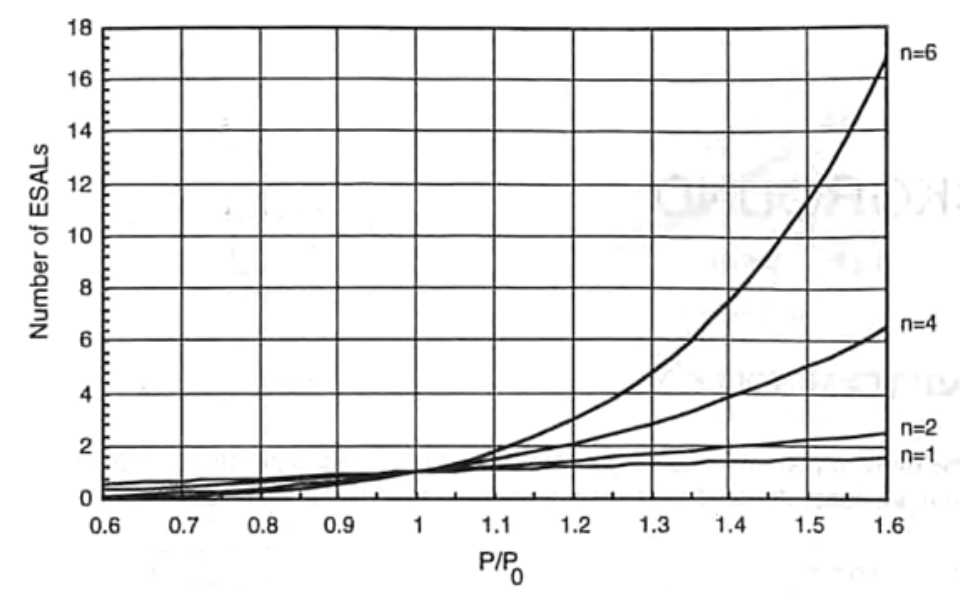

Figure 41:Various values of the exponent $n$ in the power law equation and their impact. (Cebon, 1999)

Even though the Equivalent Single Axle Load procedure has been found useful in the past and accounts for prediction of failure for a pavement it does not take into account vibrations and further damage that is created from wheels encountering imperfections on the pavement surface. This contributes to the generation of dynamic tyre forces and is driven by the pavement roughness. Pavement roughness is defined as the sum of vertical oscillation movement distance of a vehicle 
which is relative to the horizontal distance travelled along the road during the test run. The main measure used is the International Roughness Index (IRI) with units usually $\mathrm{mm} / \mathrm{m}$ and it also refers to the quality of a vehicle riding on the pavement.

At this point, it is important to differentiate between the static loading influence of a vehicle and the dynamic loading influence. Static loading properties that affect pavement damage would be: the gross vehicle weight, the axle configuration, the static load sharing between axles as well as tyre contact conditions. On the other hand, vehicle dynamics would depend on vehicle speed, vehicle suspension and road roughness.

\section{Dynamic Loading Concept}

Sweatman (1983) introduced the concept of Dynamic Load Coefficient (DLC) in order to take into account the effect of the dynamic wheel loads. This coefficient can account for different axle suspension types and is basically the coefficient of variation of dynamic wheel forces which are relative to the static forces (Davis and Bunker, 2008). It is defined as a ratio of the standard deviation of the wheel load over the mean wheel force. The DLC depends on factors like the surface road roughness, suspension type, and vehicle speed and it is shown in the following equation:

$$
D L C=\frac{\sigma}{F}
$$

\section{$\sigma:$ Standard deviation of wheel load}

F: Mean wheel force

The DLC has been used in several occasions as part of methodologies that aim to discover the influence of vehicle dynamic loading. An attempt to research into measuring the dynamic wheel loads generated by heavy vehicles as they travel on the pavement surface was made by Eisenmann et al. (1987) as is described in a published report by TRL. They developed a dynamic load factor which can account for the dynamic effect of wheel loads. To do this it was assumed that the dynamic wheel distribution is random, therefore meaning that at every point of the pavement the same wheel load distribution would be encountered, and it is based on the fourth power value. The calculation of this factor is given by the following equation:

$$
A W F=\left(1+6(D L C)^{2}+3(D L C)^{4}\right) \times(W C \times C P \times L E \times A C \times S)^{4}
$$


Where AWF: Axle wear factor (Dynamic Component x Static Load)

WC: Wheel Configuration factor

CP: Tyre Contact Pressure factor

LE: Load Equalisation factor

AC: Axle Configuration factor

S: Axle weight

\section{Aggregate damage approach}

An approach that takes into account spatial distribution of tyre forces along the pavement surface is presented by Cebon (1999) and is previously known as a 'weighted aggregate force model' (Cebon, 1993). According to his research the dynamic forces of each tyre, that are applied to each location along a pavement section, are summed up and raised to a power $n$. The following equation gives the aggregate $\mathrm{n}^{\text {th }}$ power force at location $\mathrm{k}$ :

$$
A_{k}^{n}=\sum_{j=1}^{N_{a}} P_{j k}^{n}, \quad k=1,2,3, \ldots N_{s}
$$

Where $P_{j k}$ : the force from tyre $\mathrm{j}$ at location $\mathrm{k}$

$\mathrm{N}_{\mathrm{a}}$ : Number of axles of the vehicle

$\mathrm{N}_{\mathrm{s}}$ : Number of points along the road

$n$ : Type of road damage ( $n=4$ for fatigue damage, $n=1$ for rutting both for flexible pavements).

Introducing this approach has as a result the increase of the theoretical 'damage' at certain points along the road in relativity the rest, meaning that they can define the failure of the pavement much sooner. These are called 'hot spots' and they may develop depending on fatigue failure, 'spatial repeatability', the road surface profile and road structural properties (Cebon, 1999). The use of this approach led to important discoveries like the phenomenon of spatial repeatability. The fact that trucks and vehicles have similar properties like weights, dimensions, and they travel at similar speeds, results in some of the peak tyre forces to be applied at the same locations along the road. This has been examined and verified by Cole and Cebon (1992). At this point it is not included in this research study. 


\section{Vehicle road interaction}

According to Cebon (1999) two types of studies have been found for vehicle road interaction and these are 'single-vehicle pass models' and 'whole-life models'.

Single-vehicle pass models are used when one vehicle passes a pavement and the road response is estimated at each location defining an incremental road damage after each pass. Dynamic tyre forces can be simulated due to a specific road surface profile and are used in the estimation of primary pavement responses like stresses and strains. These are then translated into measures of pavement damage, like for example the fatigue law, and the number of wheel loads to failure. Damage accumulation models like Miner's law are used also to identify the number of cycles to failure at that particular location. A similar approach is used in this research study.

The vehicle dynamic loading element is used in this research study. This would be the first attempt in combining vehicle dynamic loading with the prediction of reflective cracking in composite pavements.

\subsection{Vehicle dynamic model description}

For investigating vehicle pavement interaction and ride performance the calculation of dynamic tyre forces is necessary. This would entail simulating the road surface profile and eventually the behaviour of a vehicle model using a time domain simulation. The generation of the surface profile may be done with the spectral densities of the profile height of equally spaced points and a general method would involve the Draft ISO Formulation. This entails the use of specific parameters depending on the road class (Very Good, Good, Average, Poor, Very Poor).

Specifically, to generate a one-dimensional random profile a set of random phase angles, distributed in a uniform manner between 0 and $2 \pi$, is required and applied to a series of coefficients derived from the desired direct spectral density. Consequently, with the help of the inverse discrete Fourier transform (DFT), a series of spot heights at the equally spaced points along the pavement surface. This way a random profile is created by having an exact target spectral density.

More information regarding the vehicle model used follows, with a brief description of the dynamic vehicle model taken from Collop and Cebon (1995). 


\subsubsection{Surface profile generation}

A method according to Robson classification (Robson, 1979), is used initially to generate the profile of the pavement which will be used to determine the set of forces as a function of the distance from the start of the pavement section. This profile generation depends on certain factors like the total length of the pavement section and the average International Roughness Index (IRI) value of the section. The output of this program is the displacement profile of the surface of the pavement at data points along the pavement every $0.1 \mathrm{~m}$. According to Robson the road profile spectra can be given from the equation:

$$
S_{u}=S_{0}|\kappa|^{-n}
$$

Where $n=2.5$ and $S_{0}$ is given depending to which class the road belongs: Motorway, Principal road, Minor road.

In our case we are referring to Motorways and the value of $S_{0}$ would be in the range of 3-50/10 ${ }^{8} \mathrm{~m}^{0.5} \mathrm{cycle}^{1.5}$. This spectral density is input to the equation of a one-dimensional random profile which will generate surface heights at specific interval points along the pavement.

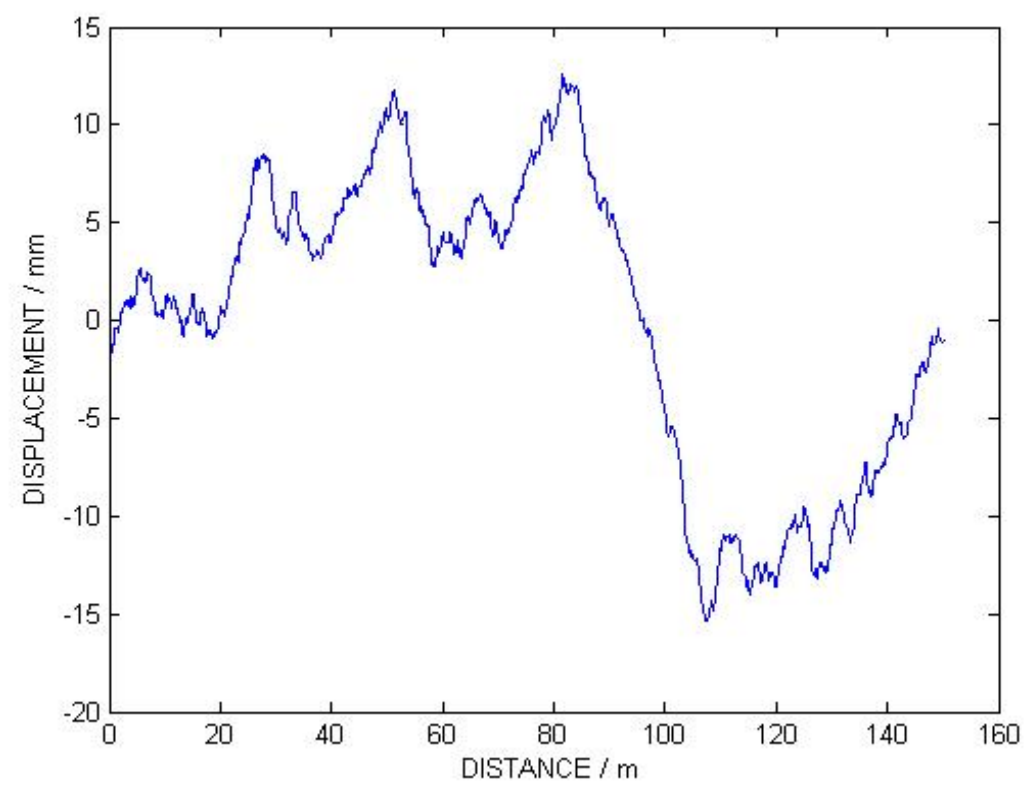

Figure 42: Example of road surface profile. 
In Figure 42 above an example of a surface profile is given for a typical 150m pavement section. On the $\mathrm{y}$ axis is the displacement measured in $\mathrm{mm}$ and it is given as a function of distance measured in $\mathrm{m}$. This surface profile will be used along with a vehicle simulation to generate the dynamic tyre forces.

\subsubsection{Quarter-car model}

A useful way to simulate a vehicle or part of a vehicle is with the use of a quarter car model. A quarter car model depicted in Figure 43, as the name reveals, represents a quarter of a car and can describe the general vibration characteristics of heavy vehicle suspensions. It has two-degrees of freedom movement and it consists of a sprung and unsprung mass $\mathrm{m}_{\mathrm{s}}$ and $\mathrm{m}_{\mathrm{u}}$. Both masses are supported by a relevant spring stiffness $\mathrm{k}_{\mathrm{s}}$ (sprung) and $\mathrm{k}_{\mathrm{t}}$ (unsprung) and a shock absorber with a damping coeffiient $\mathrm{C}_{\mathrm{s}}$ (sprung) and $\mathrm{C}_{\mathrm{t}}$ (unsprung) which is in constant contact with the surface of the pavement. The road profile in the figure is symbolized $\mathrm{v}$ and is input to the tyre. The output from that is the tyre dynamic forces that are generated at each specific point along the surface.

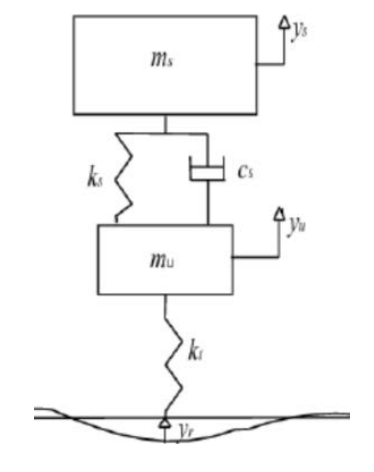

Figure 43: Quarter-car model.

Cebon (1993) noted that a simple linear 'quarter car' vehicle model can be used to represent dynamic properties of the majority of single axle truck suspensions. The vehicle parameters used in our case are the following:

$\mathrm{cs}=40 \mathrm{kNs} / \mathrm{m}$;

$\mathrm{ct}=4 \mathrm{kNs} / \mathrm{m}$;

$\mathrm{ks}=2 \mathrm{MN} / \mathrm{m}$;

$\mathrm{kt}=3.5 \mathrm{MN} / \mathrm{m}$; Cebon (1993) 
A time domain vehicle simulation is used to generate dynamic tyre forces as a function of distance along the pavement. To estimate the dynamic forces applied on the pavement due to a vehicle model, numerical integration of the equations of motion of the vehicle model take place, by inserting as input to the tyre the surface pavement profile that was generated. The equation of motion of vibrations of a linear vehicle model (Cebon, 1999) used here written in matrix form is:

$$
[M] \ddot{q}+[C] \dot{q}+[K] q=Q
$$

Where q: vector of generalised coordinates,

Q: vector of generalised forces

$[\mathrm{M}]$ : the mass matrix

[C]: the damping matrix

$[\mathrm{K}]$ : the stiffness matrix

The output from this equation is the generation of vehicle dynamic tyre forces as a function of distance along the pavement. An example from the program is plotted in the following figure (Figure 44). The three different coloured lines represent a different set of forces generated by a quarter-car model of different weight each time, but for the same road profile. The difference between them are the values of the sprung and unsprung masses with red being the heaviest and blue the lightest.

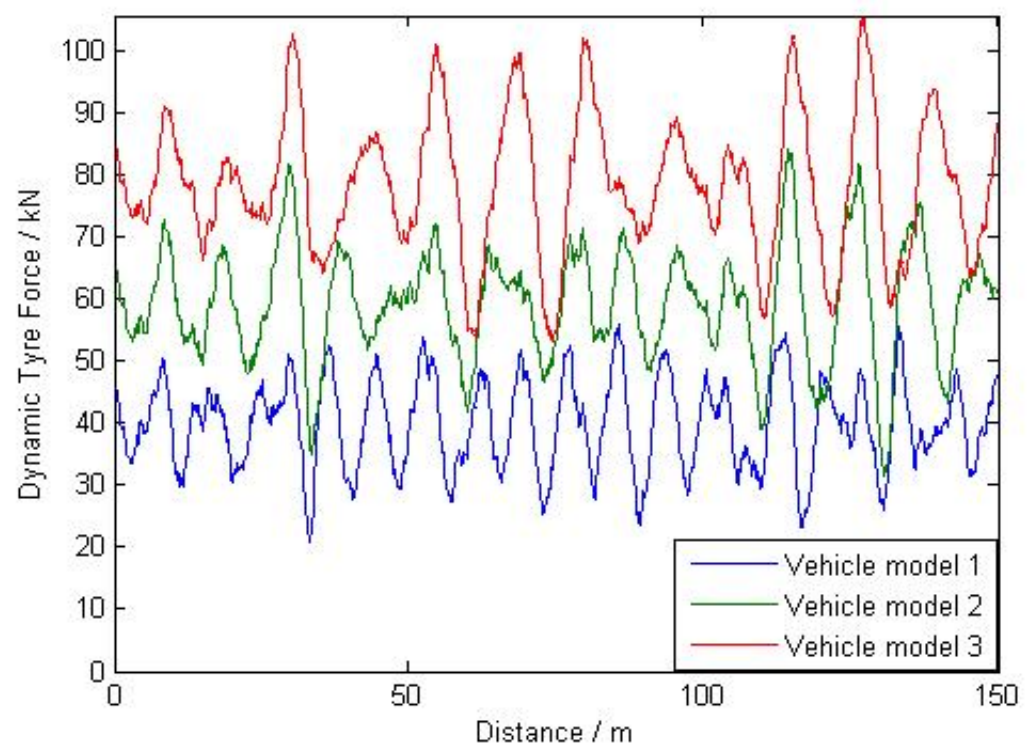

Figure 44: Example of dynamic tyre forces of three different quarter-car models. 


\subsection{Dynamic vehicle model and reflective cracking}

The developed reflective cracking model is applied with both static wheel loading and dynamic vehicle loading. When the model is combined with static wheel loading then one specific wheel load of $40 \mathrm{kN}$ would be applied on the pavement and it would not vary according to the pavements roughness. All the potential locations that can develop reflective cracking will show its appearance at the same time. By applying dynamic loading, depending on the IRI of the pavement section a set of forces as a function of distance on the pavement section is estimated.

\subsubsection{Flowchart and description}

The flowchart in Figure 45: Vehicle dynamic loading flowchart. Figure 45 reveals the dynamic model processes as well as the way they are combined in a simplified manner with the reflective cracking model. The first thing required is the input which includes an initial International Roughness Index value for the pavement, the length of the whole pavement section under examination, the crack spacing, the vehicle speed and the values of the sprung and unsprung masses.

The first process of the model as was described earlier, is the generation of the surface displacement profile which depends on the IRI value and the pavement section length. The profile is then used to estimate the force distribution with the help of a quarter-car model of a specific weight at a specific vehicle speed. The extraction of the forces at the critical locations follows. These dynamic tyre forces, where reflective cracks can develop (locations where underlying cracks or joints existed), are used as a wheel load in the reflective cracking model and lead to a bottom-up crack and top-down crack at each location. This procedure is repeated for the number of vehicle models included in the program. In this case they are $9\left(\mathrm{~N}_{\mathrm{v}}\right)$ different vehicle models that are used in the program. They represent a different weight and different speed of the vehicle. Therefore, 9 different sets of forces are estimated due to each vehicle model, and 9 cracks (bottom-up and top-down) at each potential location are estimated.

After an amount of time set in the program $\mathrm{N}$, (for example 1 month of iterations) the cumulative sum of cracking in both directions is calculated and it is checked how many locations have completely cracked, meaning reflective cracking has developed. This number of reflective cracks is inserted in a correlation equation extracted from data in the LTPP database, that is a function of the measured transverse cracks on the surface of the asphalt layer and a new IRI value is estimated. This new value 
of IRI is fed back into the program and a new surface profile is generated, leading to a new set of forces estimated.

The whole process is repeated until all locations develop reflective cracking or until the amount of traffic data inserted in the program runs out.

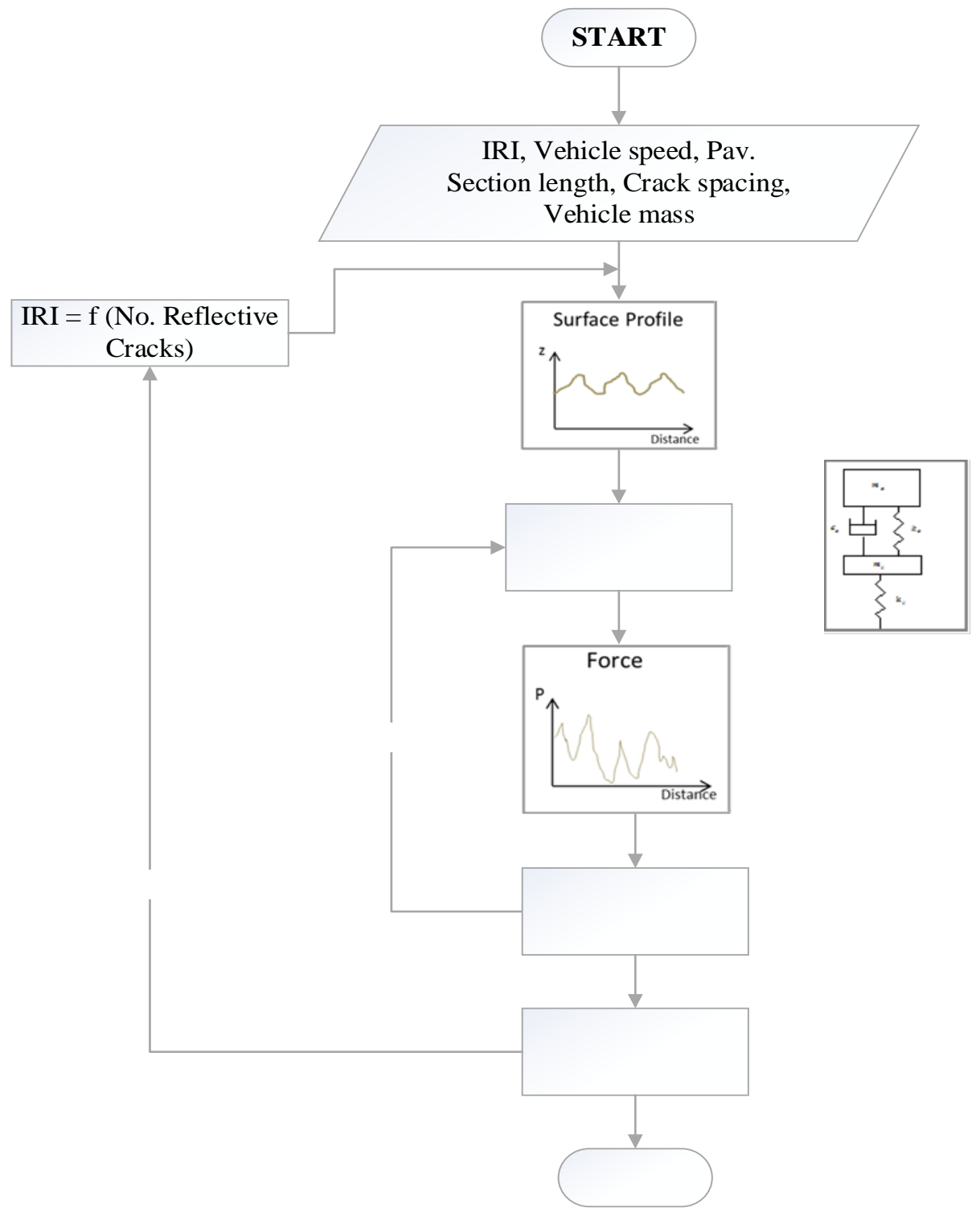

Figure 45: Vehicle dynamic loading flowchart. 


\subsubsection{Vehicle models}

In the Long-Term Pavement Performance database, the group of axles are given in 40 different weight groups. The average of 10 consecutive weight groups is estimated starting from the first 10 , in order to create broader weight groups and make the application of the model easier resulting in 4 groups (Table 13). First a conversion took place from axle groups to wheel weight groups. From these, the first one was considered to be light and after experimenting and assessing the results it was concluded that there was no effect on the results from that group. The other 3 average weights (Table 14) represent the different weight groups used in the program. These are inserted each time in the vehicle dynamic loading model as a total sprung and unsprung mass and they are used to calculate the forces that are applied on a $150 \mathrm{~m}$ length pavement section.

Table 13: Total mass of wheels.

\begin{tabular}{|c|c|c|c|c|}
\hline & $\mathbf{1}$ & $\mathbf{2}$ & $\mathbf{3}$ & $\mathbf{4}$ \\
\hline Mass (kg) & 1112 & 3336 & 5560 & 7784 \\
\hline
\end{tabular}

Table 14: Three selected wheel mass groups.

\begin{tabular}{|c|c|c|c|}
\hline & $\mathbf{1}$ & $\mathbf{2}$ & $\mathbf{3}$ \\
\hline Total mass $(\mathbf{k g})$ & 3336 & 5560 & 7784 \\
\hline
\end{tabular}

The dynamic vehicle loading works with 9 vehicle models which are based on three different weight groups (1, 2, 3 in Table 14) at three different vehicle speeds (96.6 km/h, $104.6 \mathrm{~km} / \mathrm{h}, 112.6 \mathrm{~km} / \mathrm{h})$. The speeds were selected based on speed distribution information reported in LTPP reports. The reports published vehicle speed distributions for certain pavement sections. The vehicle speed groups that had the higher percentages of vehicles were found to be $60 \mathrm{mph}, 65 \mathrm{mph}$ and $70 \mathrm{mph}$. An example of this distribution can be found in (ARA, 2012). The 9 vehicle models with the corresponding weight and vehicle speed are shown in the following table.

Table 15: Vehicle models (speed and mass).

\begin{tabular}{|c|c|c|}
\hline Vehicle models & Speed $(\mathbf{k m} / \mathbf{h})$ & Total mass $\mathbf{( k g )}$ \\
\hline $\mathbf{1}$ & 96.6 & 3336 \\
\hline $\mathbf{2}$ & 96.6 & 5560 \\
\hline $\mathbf{3}$ & 96.6 & 7784 \\
\hline $\mathbf{4}$ & 104.6 & 3336 \\
\hline $\mathbf{5}$ & 104.6 & 5560 \\
\hline $\mathbf{6}$ & 104.6 & 7784 \\
\hline
\end{tabular}




\begin{tabular}{|l|l|l|}
\hline $\mathbf{7}$ & 112.6 & 3336 \\
\hline $\mathbf{8}$ & 112.6 & 5560 \\
\hline $\mathbf{9}$ & 112.6 & 7784 \\
\hline
\end{tabular}

\subsection{Summary}

In this chapter a differentiation between static and dynamic vehicle loading was initially made. A very important discovery used in pavement design in the biggest part of the $19^{\text {th }}$ century was the 'Equivalent Standard Axle Loads' as a measure of influence on pavement serviceability. However, even though it simplifies calculations and it was a common base for researchers and designers, the fact that it depends on a single static load did not make it reliable enough. It did not take into account road roughness, vehicle dynamics, and the pavement's profile. The need to a replacement led to the investigation into dynamic vehicle loading, which is believed to reveal a quicker damage on the pavement at certain locations, depending on the surface profile.

Vehicle dynamic modelling was incorporated in whole life pavement models. The current one is based on work by Collop and Cebon (1995). It includes surface profile generation, dynamic tyre forces calculation and the goal is to simulate a whole vehicle fleet and investigate the effect on the pavement damage.

Based on the availability of data in the Long-Term Pavement Performance database the vehicle axle data used was arranged for simplicity reasons into three weight groups. Also, IRI correlation equations were inserted in the program, relevant to each state under study. IRI was updated after each time increment to account for the appearance of reflective transverse cracks along time revealing therefore the pavement's roughness deterioration with the appearance of reflective transverse cracks. 


\section{Whole life long-term flexible composite pavement model}

\subsection{Whole Life Pavement Performance Models - Previous work}

'Whole-life' models attempt to predict damage caused by real time traffic along with environmental or seasonal factors such as temperature or freezing (Collop and Cebon, 1995). The sort of damage to be predicted can be fatigue damage, rutting, and generally deterioration of the structure of a pavement or the surface profile with time (Cebon, 1999) with also the possible inclusion of some sort of repeatability of the generated wheel loads. A few 'whole-life' models are reviewed briefly here.

Brademeyer et al. (1988) developed a method to analyse the effects of repeating randomly applied dynamic loads on flexible pavements. The linear elastic solver VESYS IIIA was used to model single and tandem loads. With the help of pavement primary response influence functions, the level of damage cause to the pavement was calculated regarding parameters such as rutting, cracking and serviceability. The correlations between measured data on test sections and predictions from the model were strong.

Papagiannakis et al. (1990) used the same program, VESYS IIA assuming also spatial repeatability and not randomly allocated loads. This would mean that at specific points along the road the induced dynamic loads would be higher than average. The dynamic tyre forces were a result of a regression equation which would relate the coefficient of variation of dynamic tyre forces to the roughness of the pavement. The standard damage programs of VESYS IIIA were used to predict rutting and fatigue cracking. Fatigue cracking would be the damage mechanism that would affect the pavement roughness and lead to its increase over time.

Ullidtz et al. used a linear quarter car model to calculate dynamic tyre forces. The pavement structure was divided into subsections being defined by a material stiffness and thickness. The factors modelled in regards to pavement performance were fatigue roughness and surface rutting. The Odemark method of equivalent thicknesses was also utilised to reduce the layer elastic systems to an elastic half space. This was followed by the use of Boussinesq's equations to estimate stresses, strains and 
displacements within the desired layer in each subsection. The damage functions included were the total permanent deformation, resulting from surface rutting, as well as fatigue damage with the reduction of the asphalt layer modulus and the update of the asphalt layer profile.

Another whole life pavement performance model is the Whole Life Flexible Pavement Performance Model that was developed by Collop and Cebon (1995). This takes into account vehicle dynamic loading, environmental effects and road properties, and can model certain distresses such as surface roughness, rutting and stiffness degradation. However, the model is only valid for fully flexible pavements. Pavement primary response functions like strains, stresses and displacements are combined with dynamic tyre forces which are generated using vehicle simulations. This results in pavement primary response histories at regular points along the pavement which in turn can be translated into pavement damage with the use of appropriate deterioration models such as fatigue and rutting. The damage is incremental and the pavement surface profile along with the material values are updated after each pass of a single vehicle load. This procedure is continued until the pavement reaches the end of its life. What makes this model unique is that it takes into account the 'weak' spots on the pavement which are due to asphalt layer thickness variations and the 'hot' spots which are due to peak dynamic vehicle loading. The pavement surface degradation uses a deterministic approach rather than a statistical.

\subsection{Whole life long-term flexible composite model description}

As initially stated the purpose of this research project is to predict reflective cracking in composite pavements. To achieve that, a program was developed that predicts the appearance and development of reflective cracks over time on pavement sections among different states in the USA. The goal is to compare the predictions output from the program with the real measured data of reflective cracks on these pavement sections.

The different models that have been presented until now are combined in a whole-life model for the prediction of reflective cracking in flexible composite pavements. Specifically, this incorporates the reflective cracking models (bottom-up and top-down) and the dynamic vehicle model along with feedback mechanisms that allow the update of properties such as the asphalt elastic modulus with damage progression and the International Roughness Index (IRI). 


\subsubsection{Model framework}

For the model to work certain constituents are required and are described below:

\section{Input data}

Initially the required input for the model depends on the selected pavement section under examination. This input data includes:

- Layer properties (asphalt elastic modulus, asphalt thickness, concrete elastic modulus, concrete layer thickness, subbase elastic modulus, subbase layer thickness and the subgrade elastic modulus)

- The daily air temperature of the region under examination

- $\quad$ The equation of correlation between IRI and the number of transverse cracks

- The relevant daily traffic data of the specified pavement section

\section{Mechanisms}

The distress of reflective cracking as was explained is a complex one and involves the development of cracking through the thickness of the asphalt layer in two directions. The two mechanisms were explained in Chapter 2 ('Modelling of reflective cracking') and are distinguished by the different critical positions of the wheel load in relation to the crack or joint between the underlying slabs which would essentially cause two opposite directions of cracking. They are based on fatigue law relationships, relating the crack propagation rate to the power of the maximum tensile strain in the region of the crack tip. While the program is running both bottom-up and top-down cracks propagate at the same location, until they meet, meaning a reflective crack has developed at that location.

\section{Asphalt modulus update}

Crack propagation is included in the model, and it also takes into account the reduction of the effective asphalt modulus because of the increase of the crack through the asphalt thickness. This is done through a mechanism that degrades the asphalt modulus after a new crack development with the help of the compliance of the asphalt. The procedure is explained in Chapter 2 ('Modelling of reflective cracking'). 


\section{Temperature correction of asphalt elastic modulus}

Due to the characteristics of the asphalt and its thermal susceptibility, it is a material whose stiffness is dependent on temperature. At different temperatures the elastic stiffness of a specific asphaltic material will vary, taking higher values at lower temperatures. For this reason, a temperature correction of the asphalt elastic modulus is necessary to take into account the daily temperature fluctuations.

The original elastic moduli values $\left(\mathrm{Mr}_{\mathrm{r}}\right.$ of the first year after the asphalt overlay placement are inserted as input data in the program. By inserting them, a reference to the period immediately after the placement of the asphalt overlay is made. The inserted values initially are not temperature corrected to a specific temperature. This is achieved with the help of daily average air temperature data. Therefore, after each day (or time increment) the elastic moduli values of the asphalt are corrected according to the relevant temperature. The procedure which is followed to accomplish this, is taken from the LTPP Guide to Asphalt Temperature Prediction and Correction in the Federal Highway Administration Research and Technology website. The following formula is used to calculate the factor with which the previous elastic modulus is multiplied to estimate the temperature corrected value.

$$
\begin{gathered}
\text { Slope }=\log \left(\mathrm{M}_{\mathrm{r}}\right)=\text { intercept }+ \text { slope } \mathrm{T} \\
\text { ATAF }=10^{\text {Slope } \times(\mathrm{Tr}-\mathrm{Tm})} \\
\mathrm{E}=\mathrm{E}_{\text {before }} \times \mathrm{ATAF}
\end{gathered}
$$

Mr: Original asphalt elastic moduli values (MPa)

ATAF: Asphalt Temperature Adjustment Factor

$\mathrm{T}_{\mathrm{r}}$ : Reference Temperature, ${ }^{\circ} \mathrm{C}$

$\mathrm{T}_{\mathrm{m}}$ : Measured Temperature, ${ }^{\circ} \mathrm{C}$

Ebefore: Asphalt Modulus (MPa) before temperature correction

E: Asphalt Modulus (MPa) after temperature correction

The slope in the above equation depends on the aggregate properties and the characteristics of the binder. This varies usually between -0.013 to -0.032 . The slopes are negative due to the fact that the elastic moduli decrease while the temperatures increase. The FWD measurements used for the 
calculation of the slope refer to the mid-lane measurements and not the outer wheel path. That is done in order to eliminate the effect of the wheel loading and to consider just the effect of the temperature.

Once the slope is calculated, it is input in the program to calculate the Asphalt Temperature Adjustment Factor (ATAF) daily (or as often as the selected time increment requires) with Eq. (5.2). The reference temperature used is $20^{\circ} \mathrm{C}$ and every elastic modulus is corrected to this temperature. The ATAF factor consequently is used to correct the elastic modulus of the asphalt by using Eq. (5.3).

The available data from the LTPP regarding temperature, is the air temperature at each pavement section. An empirical equation is used to estimate the temperature of the asphalt pavement layer from the air temperature (Collop and Cebon, 1995):

$$
\mathrm{T}_{\text {pav }}=\mathrm{T}_{\mathrm{air}} \times\left(1+\frac{76.2}{\mathrm{~h}+304.8}\right)-\frac{84.7}{\mathrm{~h}+304.8+3.3}
$$

Where $\mathrm{h}$ is the asphalt thickness in $\mathrm{mm}$.

\section{Loading rate asphalt modulus correction}

The asphalt elastic modulus depends also on the loading rate, therefore, a correction according to the loading rate is also included in the program. This can be seen from the following formula (Ullidtz, 1979) where the prediction of the stiffness of the binder depends on parameters such as the loading rate $(\mathrm{t})$, the penetration index (PI) of the binder, the softening point (SP) and the temperature (T) (Brown et al., 1975):

$$
\mathrm{E}_{\text {binder }}=1.157 \times 10^{-7} \times \mathrm{t}^{-0.368} \times 2.718^{-\mathrm{PI}} \times(\mathrm{SP}-\mathrm{T})^{5}
$$

So,

$$
E_{\text {asph }}=f\left(t^{-0.368}\right)
$$

In order to correct an elastic modulus then it would be multiplied by a factor:

$$
\mathrm{E}_{\mathrm{cor}}=\text { factor } \times \mathrm{E}_{\mathrm{asph}}
$$

This factor would depend on the current loading pulse and the one during an FWD testing because that is where the backcalculated values originate from. According to a publication of Federal Highway 
Administration, during FWD testing the load pulse is in the range of 15-35ms. An average value would be $25 \mathrm{~ms}$ (0.025s). The factor used in Eq. (5.6) would be:

$$
\text { factor }=\frac{t^{-0.368}}{0.025^{-0.368}}
$$

The current loading time is found by the simple equation (Brunton, 1983):

$$
t=\frac{1}{V}
$$

Where $\mathrm{V}$ is the vehicle speed in $\mathrm{m} / \mathrm{s}$.

Finally, the factor for correcting the modulus due to loading rate is found and the modulus is corrected. This correction in the program happens for each different vehicle speed that is used.

\section{Dynamic vehicle model}

Incorporating a dynamic vehicle model in the whole-life model is significant as it takes into account vehicle parameters such as the weight of the axles and the vehicle speed, as well as riding conditions, like the roughness of the pavement surface. In order to represent the vehicle fleet, 9 vehicle models are used depending on their weight group and the vehicle speed. Each vehicle model run causes the cracks to propagate further and therefore, all of them contribute to the total damage.

The dynamic vehicle model which is presented in Chapter 4 ('Dynamic Vehicle Model') is used to estimate the forces that represent the wheel loads of which the vehicle fleet consists. The model is found from literature (Collop and Cebon, 1995) and is used in whole life flexible pavement model. In regards to the vehicle speed distribution selected, it was found from relative reports of the LTPP database. According to vehicle speed distributions published for pavement sections along time the most appropriate vehicle speeds were found to be $60 \mathrm{mph}, 65 \mathrm{mph}$ and $70 \mathrm{mph}$ due to most of the vehicles found to be travelling at these speeds. An example of this distribution can be found in A.R.A. (2012). Therefore, it is assumed that this speed distribution may be applied for all the pavement sections in the case studies. The distribution is estimated approximately from the available information in the reports and is given in Table 16. 
Table 16: Vehicle speed distribution from LTPP.

\begin{tabular}{|c|c|c|c|}
\hline Vehicle speed (mph) & $\mathbf{6 0}$ & $\mathbf{6 5}$ & $\mathbf{7 0}$ \\
\hline Vehicle speed (km/h) & 96.6 & 104.6 & 112.7 \\
\hline Distribution \% & 21.5 & 63 & 15.5 \\
\hline
\end{tabular}

\section{IRI \& Number of transverse cracks correlation}

The forces which are output from the dynamic vehicle model depend on certain parameters like the vehicle speed and the International Roughness Index (IRI). The appearance of reflective cracks on the surface of the asphalt layer is assumed to affect the roughness, therefore that should be taken into consideration throughout the analysis. That is why after a certain time period it is checked whether the surface shows reflective cracks that have appeared through the whole asphalt thickness, and the IRI is updated depending on that. This is done with the help of correlation equations that relate the IRI of a certain pavement section with the number of reflective cracks on its surface. As reflective cracks - or failed locations - are considered those that in the analysis the bottom up crack meets the top down crack.

In the LTPP database, the International Roughness Index (IRI) is recorded for each pavement section over periods of time. The series of these values for all the pavement sections of a state are correlated with the number of transverse cracks recorded. This correlation is included in the program and every time the number of reflective transverse cracks is updated it is fed back into the program to update the value of the IRI with the help of these correlation equations. This leads to the vehicle dynamic models producing a different set of forces to reflect the change of the IRI.

After the crack initiation and as the crack development progresses through the thickness of the asphalt layer at several locations, it is checked whether the cracks have developed completely through the asphalt. The number of locations where this happens reveals the predicted number of transverse cracks on the surface of the pavement. The International Roughness Index (IRI) in the program is correlated with the number of transverse cracks, and therefore a new value of IRI is calculated and fed back into the program. 


\subsubsection{Definition of failure of pavement section}

First, in order to understand the results and composite pavement progression of failure, it is important to define the conditions under which a failure of the pavement is assumed in this research study. Each composite pavement section would consist of an asphalt overlay over a rigid base with joints or preexisting transverse cracks. The locations where these underlying cracks or joints are present, are the potential locations for development of reflective cracking, by its definition. At each location, bottomup and top-down cracking are estimated. The frequency with which that occurs is after every time increment, until the point when the whole asphalt thickness is cracked at that location and the underlying crack has reflected. The presence of a reflective crack is assumed to be the failure of that specific location because no more cracking can take place from bottom up or from top to down. The number of single locations that have presented reflective cracks at the end of the analysis all contribute to the total failure of the pavement, and that is what actually is presented. The appearance of one or more reflective transverse cracks on the surface of a pavement section does not mean that the pavement has failed. However, a criterion that determines the failure of the pavement section has been set and that is when all locations with underlying cracks or joints develop reflective cracking the pavement failure would be graded as $100 \%$.

A progression of failure regarding all the locations is given as output by estimating the total number of locations that fail (develop reflective cracking) over time as a percentage of the total number of locations that potentially could develop reflective cracking. With this percentage it can be found whether reflective cracking developed at all potential locations or less. This takes the form of a ratio percentage:

$$
\% \text { failure }=\frac{\text { No. of reflection cracks }}{\text { Total No. of underlying transverse cracks }}
$$

When all the potential locations have developed reflective cracks through the thickness of the asphalt, the percentage will reach $100 \%$ and the program will end for the specific pavement section.

\subsubsection{Flowchart}

The flowchart in (Figure 46) reveals how the reflective cracking model is combined with the vehicle dynamic model into one whole model. The first thing to notice is that the whole program works with 
the help of two iterative time loops. The inner one consists of the frequency of the crack development calculation and therefore the frequency with which the asphalt elastic modulus is degraded due to the appearance of reflective cracks. The other is the outer time loop which is how often the International Roughness Index (IRI) is updated. This affects the dynamic vehicle model and the generation of forces. The selected time increments for a general application would be one week for the frequency of the crack development (inner loop) and one month for the frequency of the IRI update (outer loop).

The first process that takes place is regarding the selected pavement section under examination the equivalent relevant data are input in the program. Originally the outer loop followed by the inner loop would start counting cycles, but immediately before that happens a subprocess is responsible for the temperature correction of the asphalt modulus.

In the first time cycle (i) a surface profile is generated as a function of the distance from the start of the pavement section. The input required for that is the IRI and the length of the pavement section. The surface profile is then used to estimate the force distribution with the help of a quarter-car model of a specific weight at a specific vehicle speed. The extraction of the forces at the critical locations follows. These dynamic tyre forces, where reflective cracks can develop (locations where underlying cracks or joints existed), are used as a wheel load in the reflective cracking model and lead to a bottom-up crack and top-down crack at each potential reflective cracking location. This procedure is repeated 9 times due to $9\left(\mathrm{~N}_{\mathrm{v}}\right)$ different vehicle models that are used in the program. They represent a different weight and different speed of the vehicle. Therefore, 9 different sets of forces are estimated due to each vehicle model, and 9 cracks (bottom-up and top-down) at each potential location are estimated.

At this point, the subprocess of the asphalt modulus correction according to the loading rate should be noticed, and it happens in each cycle of a different vehicle model as it depends on the vehicle speed.

The cumulative sum of the cracks in both directions from all 9 models is estimated, as is shown in the figure in the flowchart with the y axis showing the level of cracking in the asphalt thickness (mm) and the corresponding locations (distance) on the $\mathrm{x}$ axis. In the end of this time cycle (i) the elastic asphalt modulus is reduced due to the appearance of cracks with this procedure being explained in 


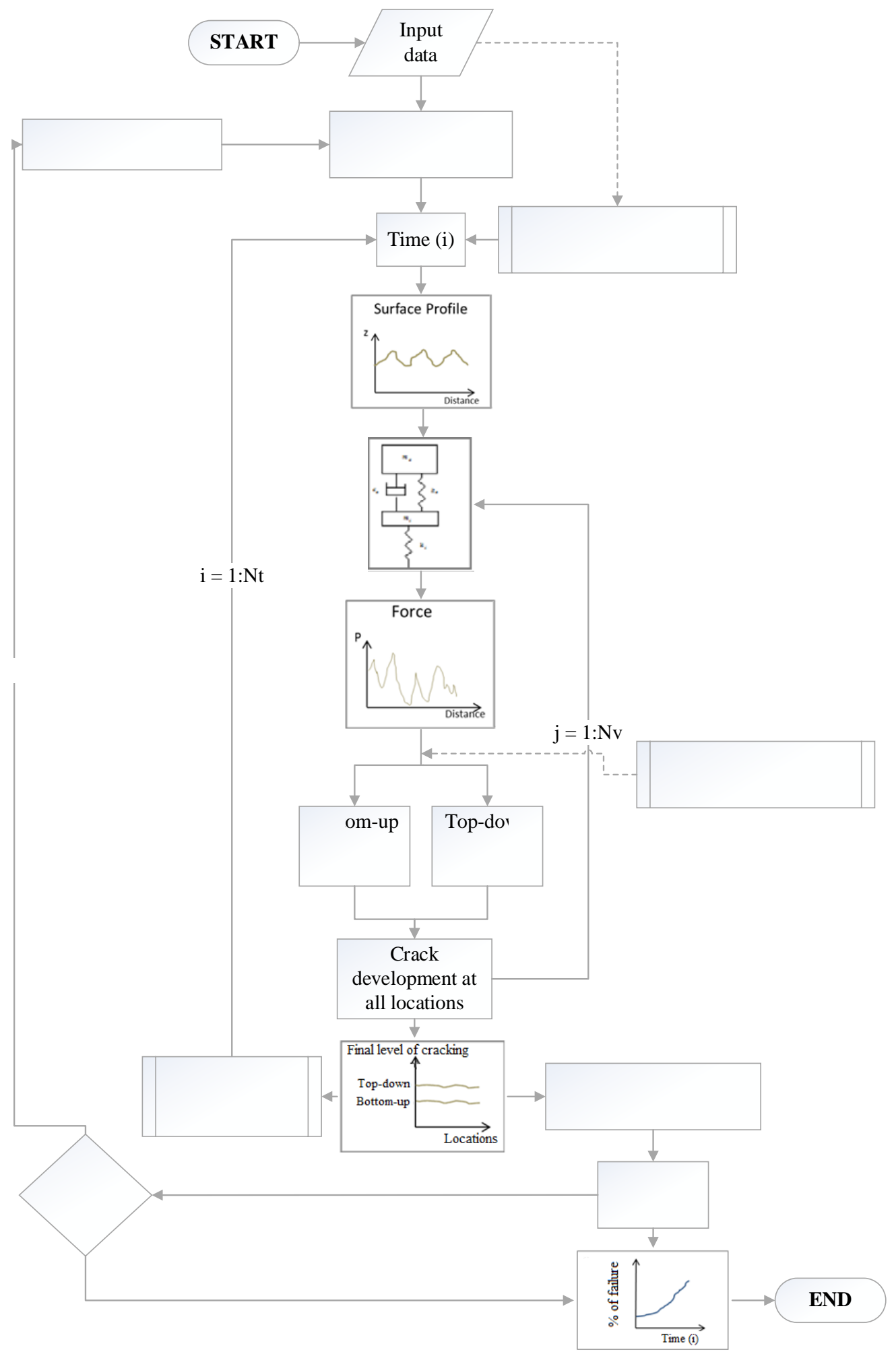

Figure 46: Whole life model flowchart.

(i: counter referring to each time increment, $\mathrm{N}_{\mathrm{v}}$ : Number of vehicle models, $\mathrm{N}_{\mathrm{T}}$ : Number of time increments) 
Chapter 2. The inner time loops continue until $\mathrm{i}=\mathrm{Nt}$, where $\mathrm{Nt}$ depends on the counter of the outer loop $\mathrm{m}$. When the outer loop is counted in months and the inner in weeks then Nt would be 4 .

When the 4 time cycles finish, the number of locations where reflective cracks have developed is checked. If failure has occurred in all the locations (100\%) then the program ends and the output prediction would be the \% of failure progression over time. If failure is less than $100 \%$ then the program continuous with the IRI update by inserting the number of reflective cracks in the correlation equation for the estimation of the new IRI. Then the next outer time loop starts and then next 4 time cycles of the inner loop.

The outer loop continuous until one of two happen:

- $100 \%$ of failure is reached (Equation 5.10)

- The counter of the outer loop m reaches $\mathrm{N}$, which represents the maximum duration of traffic data inserted in the program.

\subsection{Application of model for pavement with baseline values}

In this part of the chapter an application of the model takes place in order to show the basic steps involved. This is done for the same example pavement section as the one shown in the $2^{\text {nd }}$ chapter regarding the rehabilitated composite pavement section. The required input is shown in Table 17. Other necessary input consists of the temperature data, the traffic data and the correlation equation between IRI and number of transverse cracks on the pavement section. These are all taken from the data found regarding the pavement section of California from the Long-Term Pavement Performance database.

Table 17: Baseline pavement parameter properties

\section{Pavement property \\ Rehabilitated}

\begin{tabular}{|l|c|}
\hline Asphalt Modulus (MPa) & 4000 \\
\hline Asphalt Thickness (m) & 0.1 \\
\hline Base Modulus (MPa) & 25000 \\
\hline Base Thickness (m) & 0.2 \\
\hline Subbase Modulus (MPa) & 200 \\
\hline Subbase Thickness (m) & 0.25 \\
\hline
\end{tabular}




\begin{tabular}{|l|c|}
\hline Subgrade Modulus (MPa) & 100 \\
\hline Crack Spacing (m) & 3 \\
\hline Crack Shear Modulus (MN/m³ $)$ & 1000 \\
\hline
\end{tabular}

Below is a figure showing the weekly averaged temperature data for the state of California in the first year. This data is used for the temperature correction of the asphalt modulus, the procedure of which is shown below.

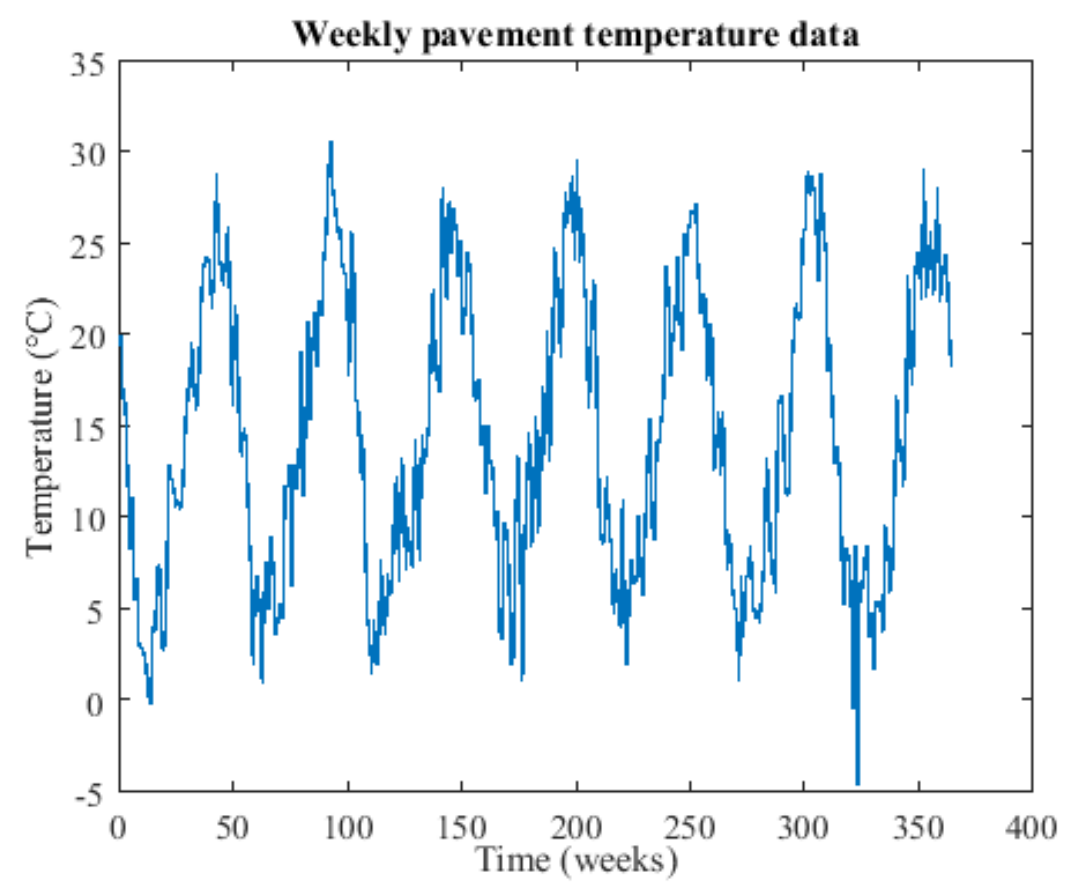

Figure 47: Weekly averaged pavement temperature over 1 year.

The temperature correction procedure is given for section 603 of the state of California for the mid-lane measurements. First the semi log graph of the elastic moduli values according to the different temperatures is given in Figure 48. The logarithmic values of the inserted elastic moduli are given on the $\mathrm{y}$ axis, according to their temperature on the $\mathrm{x}$ axis. So, the yellow line represents the equation: Slope $=\log \left(M_{r}\right)=$ intercept + slope $\mathrm{T}$. The slope in this case is -0.022 which is within the permitted range. 


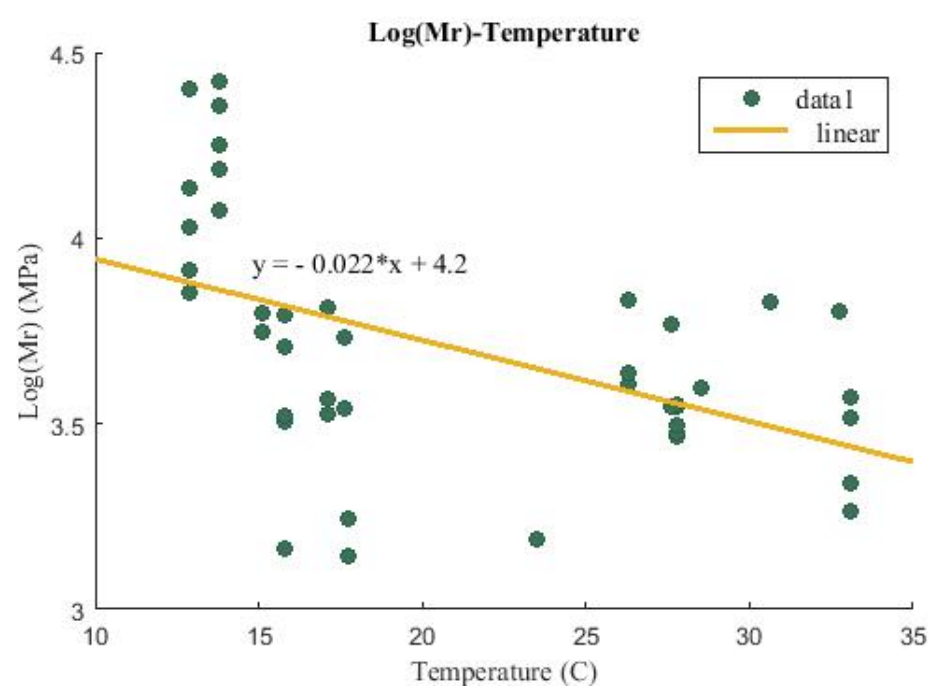

Figure 48: $\log (\mathrm{Mr})$-Temperature for estimation of slope.

The elastic modulus of the pavement section in California is $3570 \mathrm{MPa}$ before correcting it due to temperature. The average air temperature of the first week would be $14.4{ }^{\circ} \mathrm{C}$, and the asphalt thickness is $100 \mathrm{~mm}$. The following procedure (Eq. 5.4, 5.2 and 5.3) is used to correct it for the first weekly increment in the program:

$$
\begin{gathered}
\mathrm{T}_{\text {pav }}=14.4 \times\left(1+\frac{76.2}{100+304.8}\right)-\frac{84.7}{100+304.8+3.3}=20.08^{\circ} \mathrm{C} \\
\operatorname{ATAF}=10^{(-0.022) \times(20-20.08)}=1.0041 \\
\mathrm{E}=3570 \times 1.0041=3584.5 \mathrm{MPa}
\end{gathered}
$$

Before the vehicle dynamic model is applied, the number of potential locations where reflective cracking can develop are calculated. Given that the whole pavement section is 150 $\mathrm{m}$. and the crack spacing on this pavement section is $3 \mathrm{~m}$.:

$\frac{150}{3}-1=29$ potential locations

The first weekly time increment initiates with the vehicle dynamic modelling procedure and the generation of the tyre forces. The first step is the surface displacement profile estimation, which can also be seen in Figure 49. 


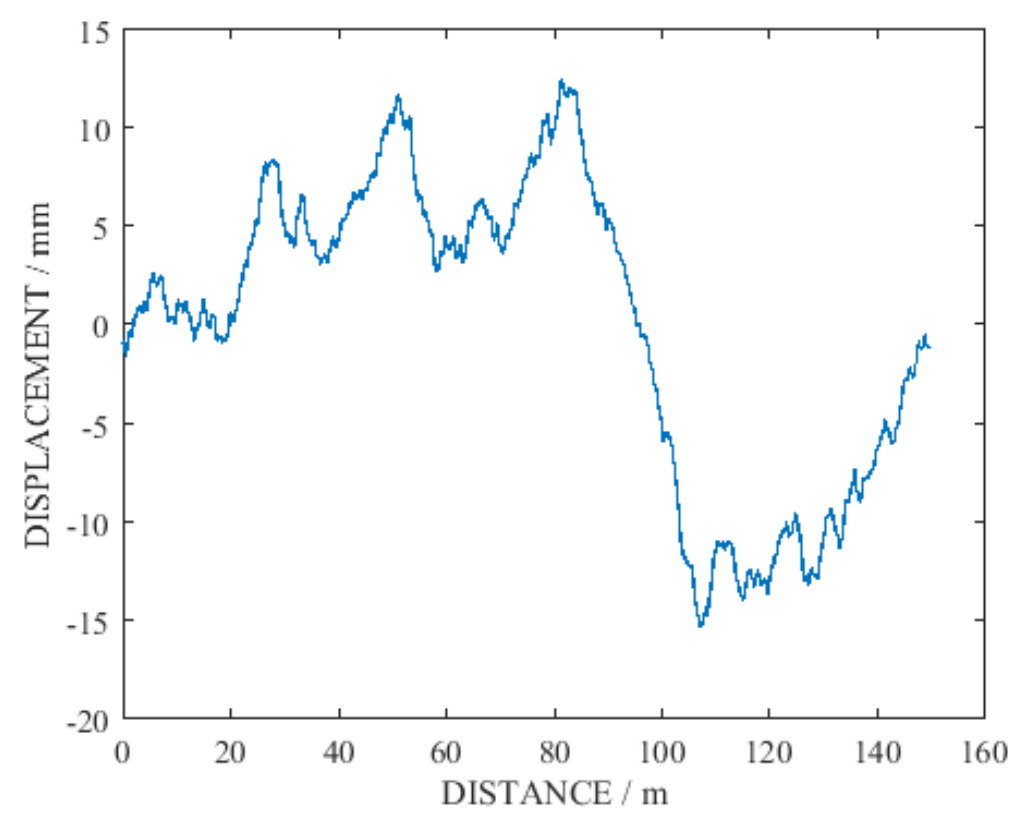

Figure 49: Surface displacement profile.

With the help of this profile, the calculation of forces for the first 3 vehicle models is achieved.

These are depicted in Figure 50 representing three different models of the same vehicle speed but different mass.

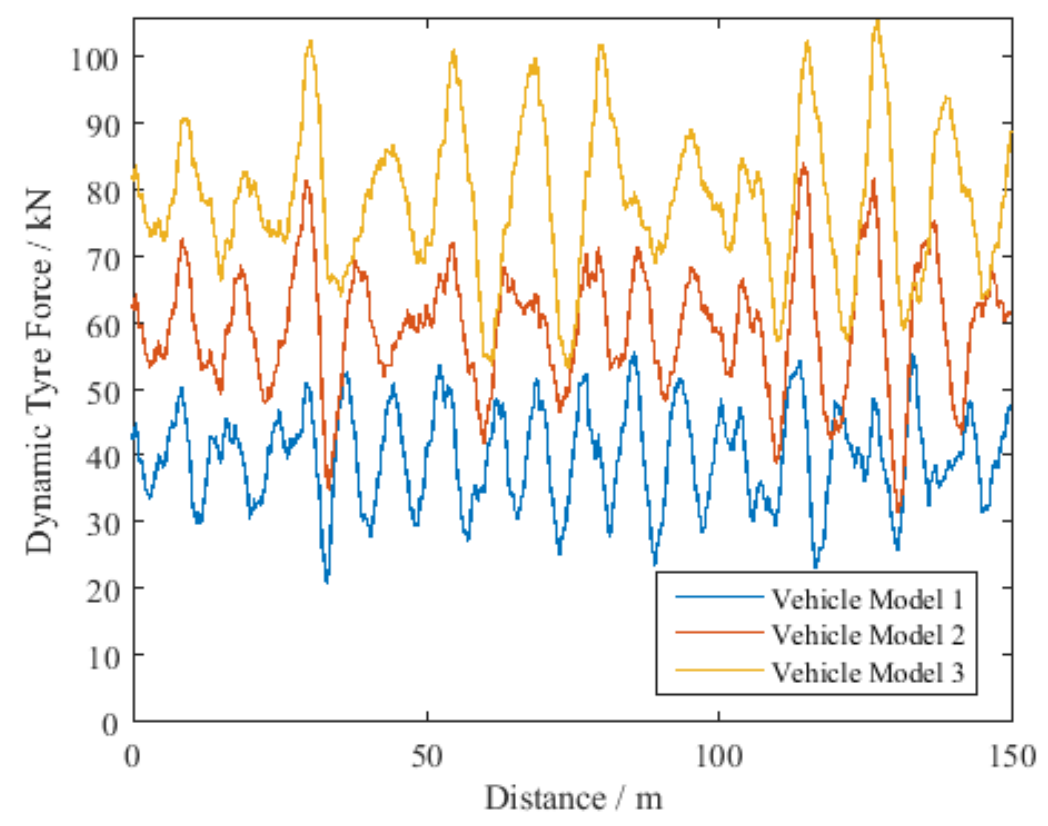

Figure 50: Set of forces representing 3 different vehicle models. 
From the above figure the forces corresponding to the specific locations where underlying cracks exist are extracted, and these locations are examined for the development of reflective cracking on the asphalt overlay.

In summary, regarding this $1^{\text {st }}$ weekly time increment: after the application of the traffic driven reflective cracking model for the $1^{\text {st }}$ location ( $3 \mathrm{~m}$. from the start of the pavement section) the outcome would be in this order for each individual vehicle model:

- Tensile strain in the region of the crack tip for bottom-up and top-down cracking

- Crack propagation rate for both directions with the help of the following fatigue law (Chapter 2, Eq. 2.7):

$$
\frac{\mathrm{dc}}{\mathrm{dN}}=A \varepsilon_{\mathrm{t}}^{\mathrm{n}}
$$

- Crack length from bottom-up and from top-down direction with the help of the following equation (Chapter 2, Eq. 2.8):

$$
\mathrm{C}=\frac{\mathrm{dc}}{\mathrm{dN}} \times \mathrm{N}
$$

For the first time increment each of the 9 models is applied and a different crack length is the outcome. The cumulative outcome of this increment is calculated for each of the 29 locations. This procedure is repeated for 4 weekly time increments when it is checked whether some of the locations have cracked completely through the asphalt thickness revealing failure in this $1^{\text {st }}$ month. The level of cracking in the asphalt layer corresponding to the $1^{\text {st }}$ location is presented in Figure 51 with the level of cracking in asphalt on the y axis and number of wheel loads on the $\mathrm{x}$ axis. It is seen that there is very little bottom-up crack development but around $19.8 \mathrm{~mm}$ crack length from the other direction.

At this point the reduction of the asphalt modulus takes place with the procedure described in Chapter 2, due to the reduction of the effective asphalt thickness.

At the end of the 4 weeks it is checked how many of the locations show reflective cracking. This is set to be done every 4 weeks. In this first month no complete reflective cracks develop. 
In this example case the first 3 reflective cracks (failed locations) would appear after 4 months (16 week iterations). The crack development in the asphalt thickness at one of the locations is shown in Figure 52.

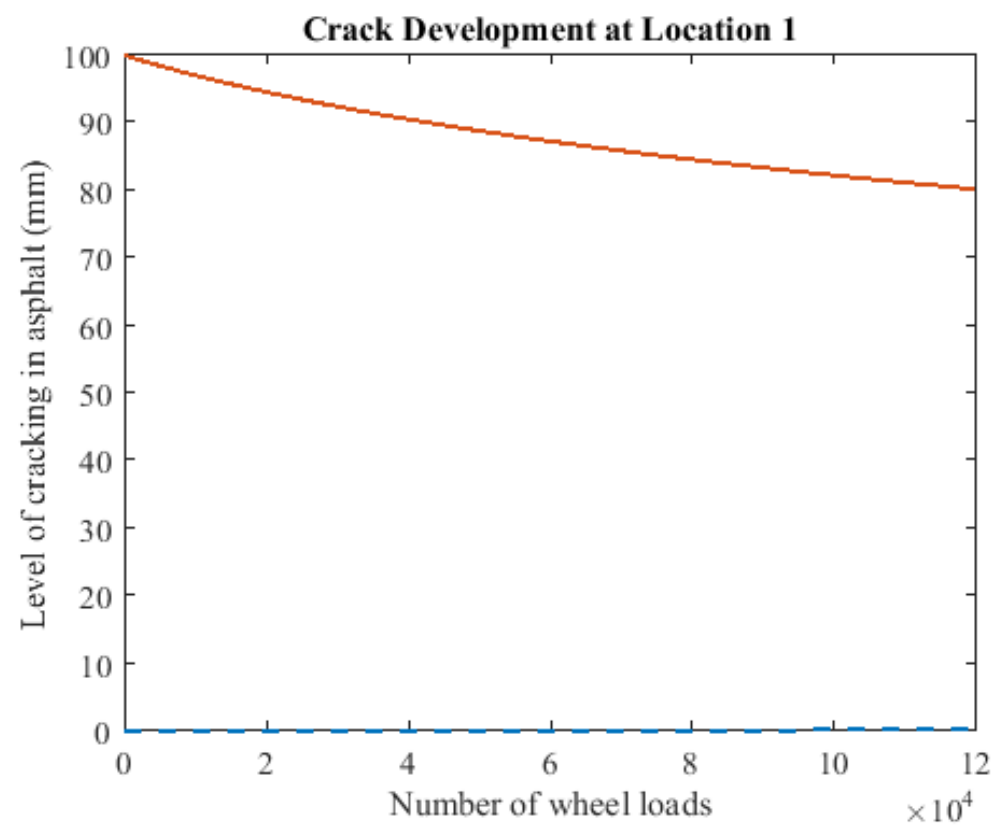

Figure 51: Crack development at 1st location (3 m. from start) after 1 month.

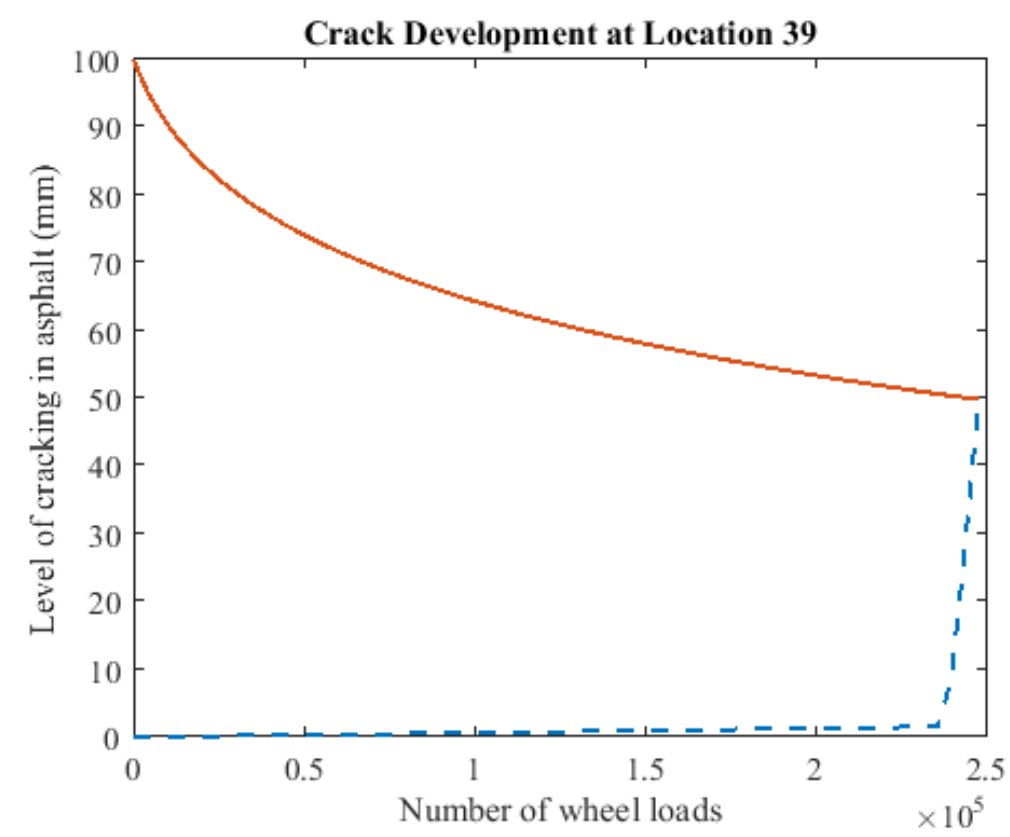

Figure 52: Crack development at location 39 (117 m. from start) after 4 months. 
At this point, due to the appearance of reflective cracks the value of the IRI would be updated with the help of the correlation equation inserted in the program. At the next step it would be fed back in for the next time increment where a new surface profile would be generated, a new set of forces etc. This procedure is repeated until all locations fail or until the traffic data inserted in the program runs out.

When the program stops all the locations have shown reflective cracks. In Figure 53 the final prediction is shown with the \% of failed locations on the $y$ axis over time (in weeks) on the $\mathrm{x}$ axis. In Figure 54 progression of failure is shown both due to dynamic and static loading for comparison.

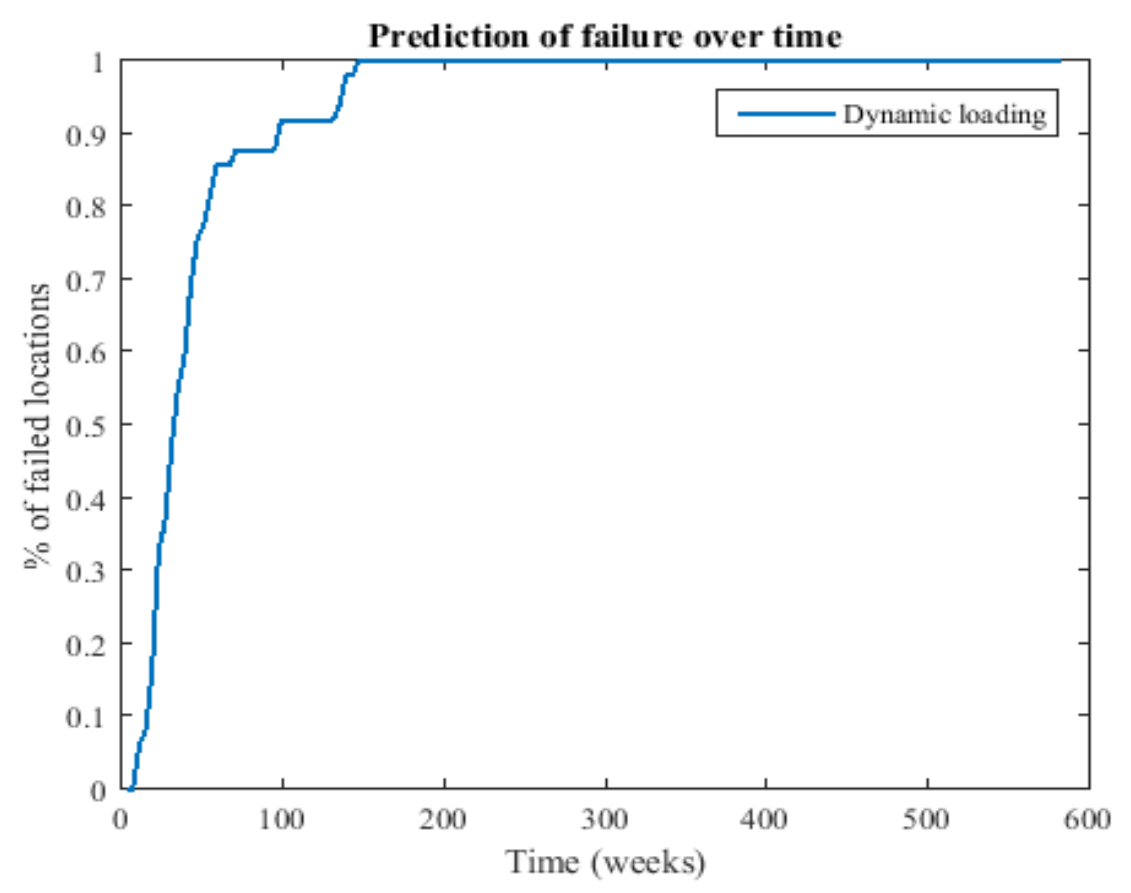

Figure 53: \% of failure over time due to dynamic loading 


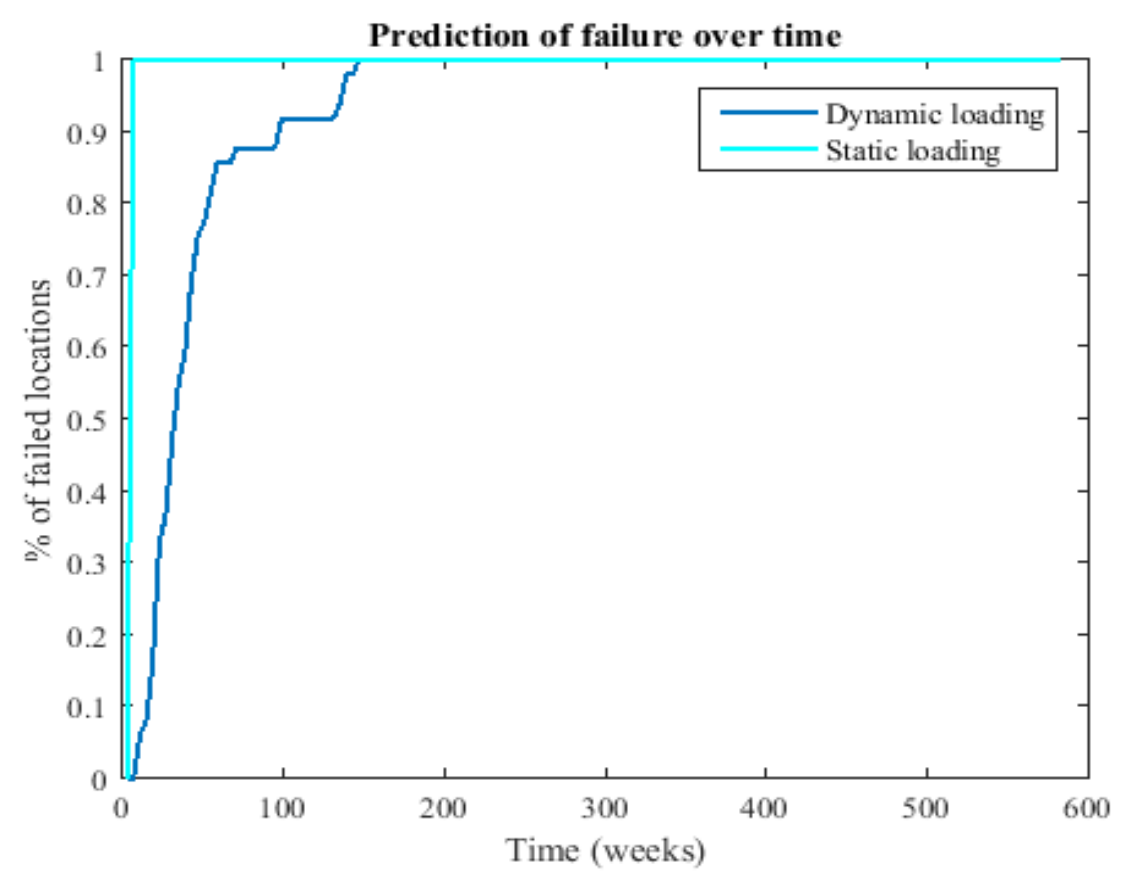

Figure 54: \% of failure over time due to dynamic and static loading

\subsection{Summary}

In this chapter the description of the whole life long-term composite pavement model is given, which regards the combination of individual models that have been analysed in the previous chapters. First of all, previous work regarding complex whole life models is presented. The description of the model follows, which consists of the framework of the model, essentially its integral parts. These are the initial input required for the program to work which consists of individual values or datasets and the basic mechanisms of the reflective cracking model, which are the bottom-up and top-down models already described in Chapter 2. In addition to them, certain processes are included regarding the asphalt modulus temperature correction, as well as its correction due to the loading rate. The dynamic vehicle model used is also mentioned here, but its full description is given in Chapter 4. Significant to the dynamic vehicle model is the input of the International Roughness Index values. The program starts initially with an IRI value, which in every time increment would be updated according to the number of reflective cracks that have developed on the pavement.

For the model to work, and according to the data availability an assumption was made in the definition of $100 \%$ failure of a pavement section. This would be when all the locations on the asphalt overlay 
that have underlying cracks or joints in the base would develop reflective cracking, meaning that the top-down crack development and the bottom-up crack would crack the whole asphalt thickness. This was considered the best solution in order to study the effect of reflective cracking.

In addition to the above, the flowchart of the whole model is given and described in detail. The program consists of two basic time loops, a weekly one at the end of which crack lengths and asphalt moduli are updated, as well as a monthly one, at the end of which the value of the IRI is updated.

Lastly, in this chapter is included a description of the application of the whole model for one example case. This was chosen to be one composite pavement section with property values representing those of a rehabilitated composite pavement section and data regarding temperature and traffic to be taken as an example from the data available regarding a section in the state of California. The whole procedure is given until the end where the prediction is shown of the \% of failed locations on this pavement section. 


\section{Parametric Study of whole-life flexible composite pavement model}

\subsection{Review of previous work}

Many researchers studied the distress of reflective cracking in an effort to identify the main factors that influence the development of this type of cracking. Kazimierowicz-Frankowska (2008), with the help of a finite-element analysis estimation of the stress field in the area of the crack, investigated the influence of different parameters on the initiation and propagation of reflective cracking. These parameters included load position, overlay thickness and subgrade properties, concluding that the position of the load in regards to its distance from the underlying crack plays a significant role in the stress development. Other researchers focusing on the influence of the overlay thickness and the thickness of the existing pavement layer on the overlay life were Elseifi \& Al-Qadi (2004). They found that by increasing the thickness of the asphalt layer from $100 \mathrm{~mm}$ to $150 \mathrm{~mm}$ the number of cycles for crack initiation also increases more than two times the original number of cycles (from 40000 to 80000$)$.

Important findings concerning the position of the load in relation to the stress field at the tip of the existing cracks in the pavement were also reported by other researchers (Pais et al., 2012), (Dave et al., 2007). According to Dave et al. (2007) the critical positions for tire loading from vehicles were considered those immediately above or very close to the pre-existing cracks in the underlying layer of the pavement. Pais et al. (2012) found that the critical position for a single crack was when the load was not immediately close to the crack but at a certain distance away, which also depends on the thickness of the asphalt layer. They developed a finite element simulation of an asphalt concrete overlaid pavement with multiple existing cracks in the underlying concrete pavement and concluded that the existence of multiple cracks does not increase the strain level in the asphalt overlay as it was first assumed. However, there are differences between the two cases (multiple cracks, single crack) regarding the stress field. 
Ni \& Li (2008) studied the development of reflective cracking in asphalt pavements with a cement stabilized base taking into account the effects of shrinkage cracking. The influence of the asphalt modulus followed by the asphalt thickness and the base modulus were found to be the most important factors controlling crack growth. Finite element analysis and fracture mechanics were used to predict the growth of reflective cracks for a specific vehicle load and radius.

In a report from TRL and the Ministry of Defense in UK (Cook and Ellis, 2005) regarding reflection cracking on airfield pavements it was concluded that the propagation of cracking is defined by several parameters. Some of those were: the brittleness of the wearing course due to low temperatures, the thickness of the asphalt layer, the resistance to age hardening as well as the temperature cycles during the pavement life.

One of the aims of this study is to identify the main parameters that influence the final results. So, in this chapter the importance of key variables on the final predictions will be investigated.

\subsection{Parametric study}

The relative importance of certain factors included in the whole model is examined, such as the selection of the time frequency with which a new crack is calculated, or the effect of individual variables that were also examined in the parametric analysis of the separate traffic driven reflective cracking model. To achieve that, certain assumptions are made for factors that would remain stable throughout the analysis, such as the daily temperature, the traffic data and the International Roughness Index (IRI) correlation equation which is necessary for the production of the dynamic vehicle forces. A standard $150 \mathrm{~m}$ pavement section with specific properties is selected to investigate the dependence of the predictions on the parameters of the whole life model. The pavement properties used as baseline values are the ones that represent the rehabilitated composite pavement case study as were used in the $2^{\text {nd }}$ Chapter. These can be seen in Table 18 as follows:

Table 18: Baseline pavement parameter properties.

\section{Pavement property}

\section{Rehabilitated}

\begin{tabular}{|l|c|}
\hline Asphalt Modulus (MPa) & 4000 \\
\hline Asphalt Thickness (m) & 0.1 \\
\hline Base Modulus (MPa) & 25000 \\
\hline Base Thickness (m) & 0.2 \\
\hline
\end{tabular}




\begin{tabular}{|l|c|}
\hline Subbase Modulus (MPa) & 200 \\
\hline Subbase Thickness (m) & 0.25 \\
\hline Subgrade Modulus (MPa) & 100 \\
\hline Crack Spacing $(\mathrm{m})$ & 3 \\
\hline Crack Shear Modulus $\left(\mathrm{MN} / \mathrm{m}^{3}\right)$ & 1000 \\
\hline
\end{tabular}

In the procedure of temperature correction of the asphalt elastic moduli, a temperature data set is selected which represents real daily air temperatures at the location of a specific state. This data set is kept constant for all the runs so it will not influence the results. It is taken from the case study of a pavement section in California.

Regarding the traffic data used, it consists of 4000 wheel loads that pass daily on the pavement section. Depending on the frequency with which a new crack develops this data is adjusted for a week, a month and a period of 3 months by averaging 7 days of traffic to represent one week, 30 days of traffic to represent one month and 90 days of traffic to represent one 3-month period.

The whole-life composite pavement model includes originally 9 vehicle models, as explained in Chapter 4 regarding the dynamic vehicle model, they represent 3 different vehicle speeds and 3 different wheel weight classes. For simplicity reasons only one vehicle model is used in the parametric analysis. The one selected belongs to the middle average weight class (5560.3 kg) and the middle vehicle speed, $65 \mathrm{mph}(104.6 \mathrm{~km} / \mathrm{h})$.

Another factor that is taken into account is the prediction of the International Roughness Index which is correlated with the number of reflective cracks on the asphalt overlay. This is assumed to be taken from the correlation equation from the California pavement section. (This is presented in Figure $7 \mathrm{~b}$ in Chapter 7 ('Validation \& Results').

\subsubsection{Convergence study}

The first thing to be examined is the effect the different time increments in the model would have on the predictions. As it was explained in Chapter 5 ('Whole life long-term composite pavement model') two time loops are built in the program. One of them, the inner loop, represents the frequency with which a new crack develops, updating the crack length development through the asphalt thickness 
which also affects the asphalt stiffness. The outer time loop represents the time frequency with which the IRI is updated, which effectively affects the forces applied by the wheels on the pavement in the dynamic vehicle model.

So, it is important to identify the ideal time increment to be used, for both loops, which would also be efficient in regards to the computation time required for a complete run of the whole life model while at the same time not compromising the accuracy of the results. Regarding the inner time loop, the different time increments tried are:

- 1 day,

- 1 week (7 days),

- 1 month (30 days) and

- 3-month period (90) days.

The time increment for the IRI update (outer time loop) would either be every 1 month or every 3 months if the above time increment is 1 day to 1 month, or it would only be 3-month if the crack development time increment is 3-month. These possible cases are shown clearly in the following table:

Table 19: Time frequency for parameter update.

\begin{tabular}{|c|c|c|}
\hline \multicolumn{2}{|c|}{ Table 19: Time frequency for parameter update. } & IRI update \\
\hline 1 & Crack development & 3-month \\
\hline 2 & 1 day & 3-month \\
\hline 3 & 1 week & 3-month \\
\hline 4 & 1 month & 3-month \\
\hline 5 & 3 -month & 1-month \\
\hline 6 & 1 day & 1-month \\
\hline 7 & 1 week & 1-month \\
\hline
\end{tabular}

The effect of the different crack development time increments for cases 1-4 is shown in Figure 55. This regards the cases with a frequency for IRI update every 3 months. In this figure the \% of locations that fully crack through the asphalt thickness over time is shown. It can be seen that time increments of 1 day (Case 1) and 1 week (Case 2) would result in the same predictions. On the other hand, time increments of 1 month (Case 3) and 3 months (Case 4) would result in similar prediction with each 
other but at the same time showing an overestimation in regards to the percentage of the failed locations, meaning that the same \% of locations would be shown to fail sooner.

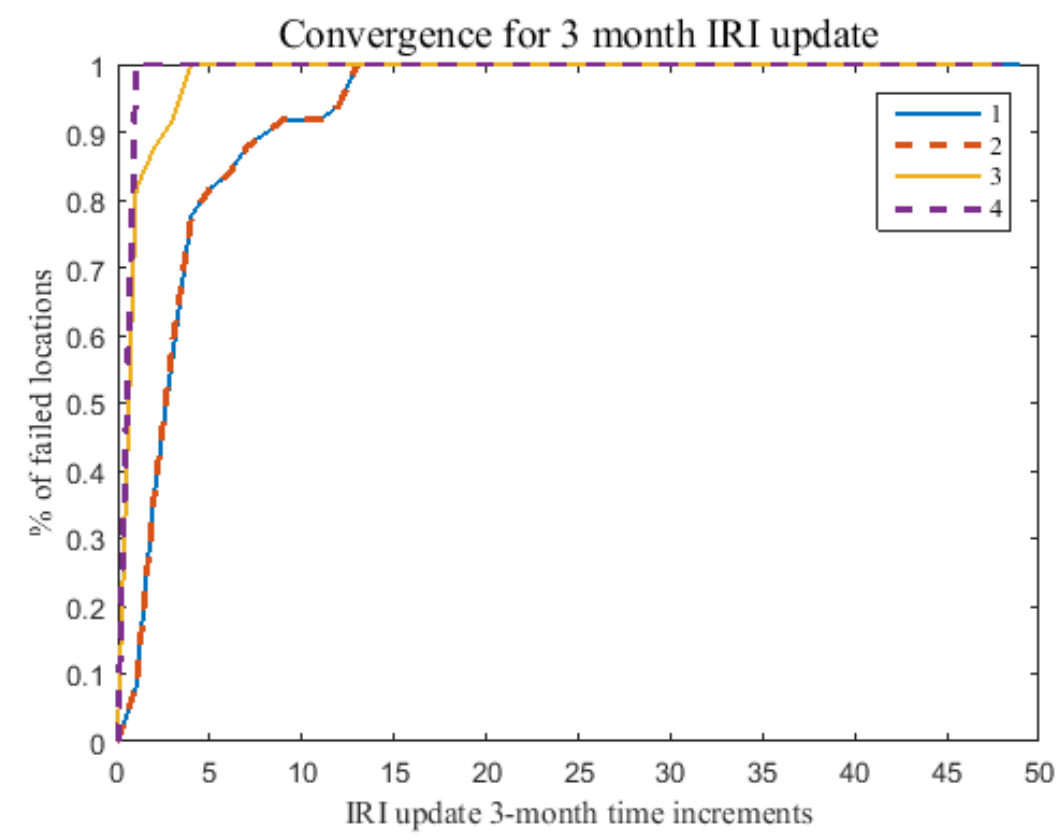

Figure 55: Failed locations over time with a 3-month IRI update

Similar predictions are shown in Figure 56 where the frequency with which the IRI is updated is 1 month (cases 5, 6 and 7). The weekly prediction (Case 6) is very similar to the daily (Case 5), while also effectively reducing the computation time. The monthly prediction (Case 7), similar to the previous figure shows failure in locations quicker. Therefore, the weekly time increment was selected as a basic time increment for the inner loop, which is how often a new crack length is estimated, due to the similarity with the daily prediction and the reduction of computation time.

Another thing checked in order to decide on the weekly time increment was examining the actual crack development at one example location of the pavement section. The $1^{\text {st }}$ potential location to develop reflective cracking was selected and the level of cracking in the asphalt layer from bottomup and from top-down is shown in relation to the number of wheel loads passing on the pavement in Figure 57. Two cases are shown of daily and weekly time increment with a 1-month IRI update frequency (cases 5 and 6 relatively). It is seen that the predictions are similar to each other, therefore, it was decided to select the weekly time increment in order to reduce computation times. 


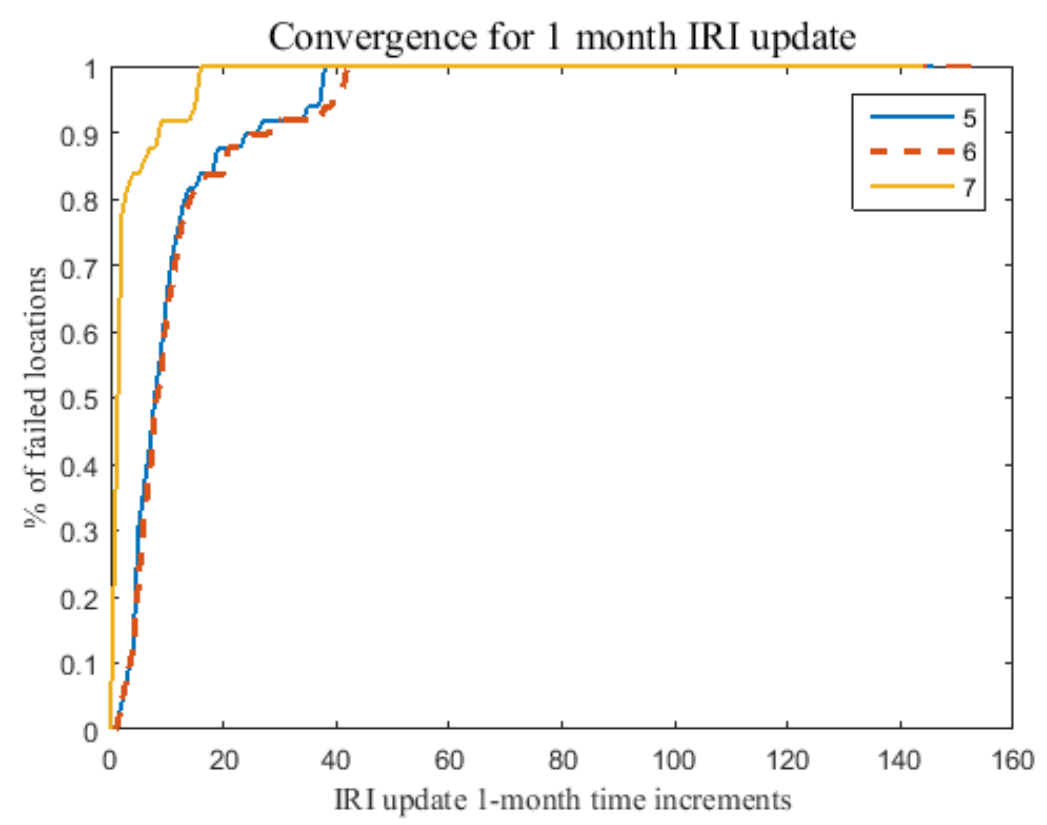

Figure 56: Failed locations over time with a 1-month IRI update

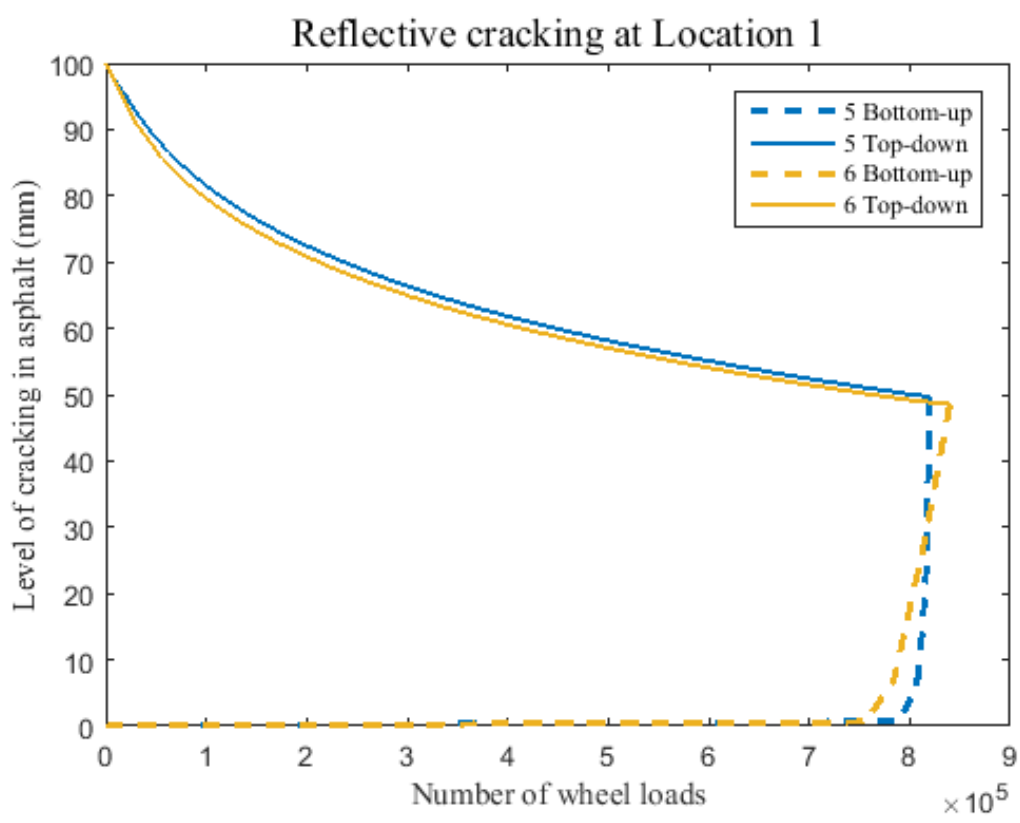

Figure 57:Reflective cracking development through asphalt thickness

In regards to selecting the frequency with which the IRI would be updated, the percentage of failure at locations is plotted along time for a weekly time increment of crack development for 1 month and 3 month IRI update in Figure 58. It is seen that there is a slight differentiation in the prediction. The 3-month IRI update frequency shows failure of locations slightly sooner than with the 1-month. 
However, the results are in general similar and the computation time does not increase for the more granular analysis 1 month. For all the above, it was decided to use the monthly IRI update increment for more reliable representation of real conditions (comparing to the 3-month increment).

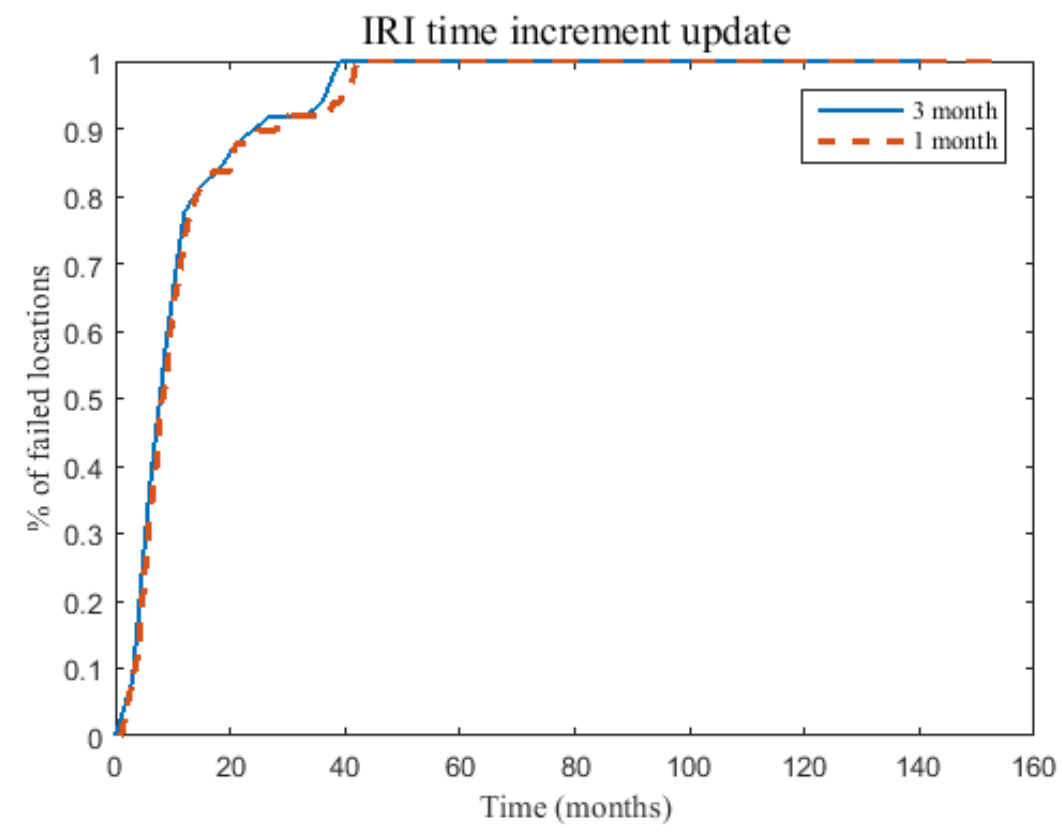

Figure 58: IRI update increment selection.

\subsubsection{Parametric analysis of specific factors}

The basic variables that can affect the outcome of the final predictions are considered to be the basic pavement structure properties. These would be the asphalt elastic modulus and thickness, the concrete elastic modulus and thickness, the subbase elastic modulus and thickness the subgrade elastic modulus. The effect of the variation of these parameters is investigated to check the influence on the predictions. The values used as a baseline are the typical ones shown in Table 18. The temperature data, traffic data and IRI correlation equations remain the same throughout all the runs as is explained above (6.2.1).

\section{Asphalt elastic modulus}

Regarding the asphalt elastic modulus variable, when varying it from the baseline values with the factors shown in Table 20 the predictions that occur can be seen in Figure 59 and Figure 60. In Figure 59 the prediction of failure for the three cases is shown, where \% of failed locations (y axis) is plotted over time (x axis). It is seen that increasing the value of the parameter would decrease the number of 
failed locations that occur at the same time. For example, 100\% of locations fail almost immediately when using $2000 \mathrm{MPa}$. However, when using $4000 \mathrm{MPa}$ 100\% of locations fail after 200 weeks of running the program and when using $6000 \mathrm{MPa} 100 \%$ is not reached in over 600 weeks (12 years). This can also be seen clearly in Figure 60 where the different plotted lines show the time required for $50 \%, 75 \%$ and $100 \%$ of the locations of a typical pavement section to fail when the asphalt modulus is being varied. In Table 20 the time required for each \% case is shown in months. For the case of $6000 \mathrm{MPa}$ it is shown in Figure 59 that it does not reach $100 \%$ of failure therefore in Figure 60 the maximum value is given which is 144 months (duration of the analysis).

Table 20: Time in months required for specific \% of failure with asphalt modulus variation

\begin{tabular}{|c|c|c|c|}
\hline Asphalt Modulus (MPa) & $\mathbf{2 0 0 0}$ & $\mathbf{4 0 0 0}$ & $\mathbf{6 0 0 0}$ \\
\hline Factor & 0.5 & 1 & 1.5 \\
\hline $50 \%$ & 2 & 10 & 47 \\
\hline $75 \%$ & 2 & 14 & 65 \\
\hline $100 \%$ & 3 & 43 & 144 \\
\hline
\end{tabular}

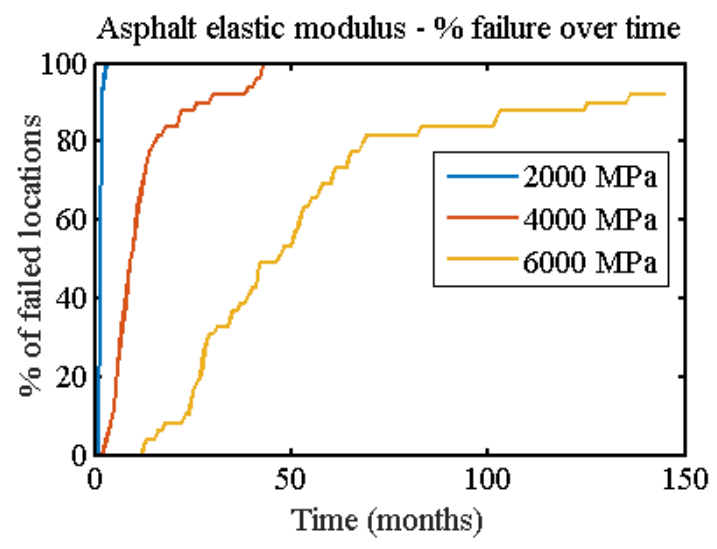

Figure 59: Asphalt elastic modulus variation - \% of failed locations over time

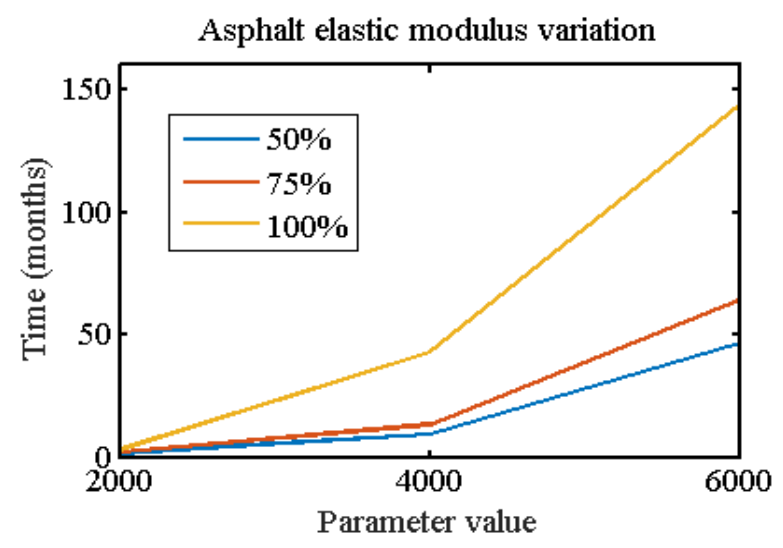

Figure 60: Asphalt elastic modulus variation

\section{Asphalt thickness}

The next parameter that was varied was the asphalt layer thickness. Same in this case as with the previous parameter, increasing the asphalt thickness would increase the time the pavement would endure before all of its locations fail (Figure 61). That is justified because the crack length at a specific location from both ends (top and bottom) would be propagating a greater distance before it would fail. In Table 21 and Figure 62 where the different times required for specific percentages of failure 
to occur in each case is shown, it is noticed that the times needed for $50 \%$ and $75 \%$ of failure do not differ by much especially in the first two cases (2000 MPa and $4000 \mathrm{MPa}$ ) whereas, there is a greater increase in the time required for $100 \%$ of failure where it is seen that it more than doubles.

Table 21: Time in months required for specific \% of failure with asphalt thickness variation

\begin{tabular}{c|c|c|c|} 
Asphalt Thickness (m) & $\mathbf{0 . 0 5}$ & $\mathbf{0 . 1}$ & $\mathbf{0 . 1 5}$ \\
\hline Factor & 0.5 & 1 & 1.5 \\
\hline $50 \%$ & 4 & 10 & 23 \\
\hline $75 \%$ & 5 & 14 & 32 \\
\hline $100 \%$ & 10 & 43 & 98 \\
\hline
\end{tabular}

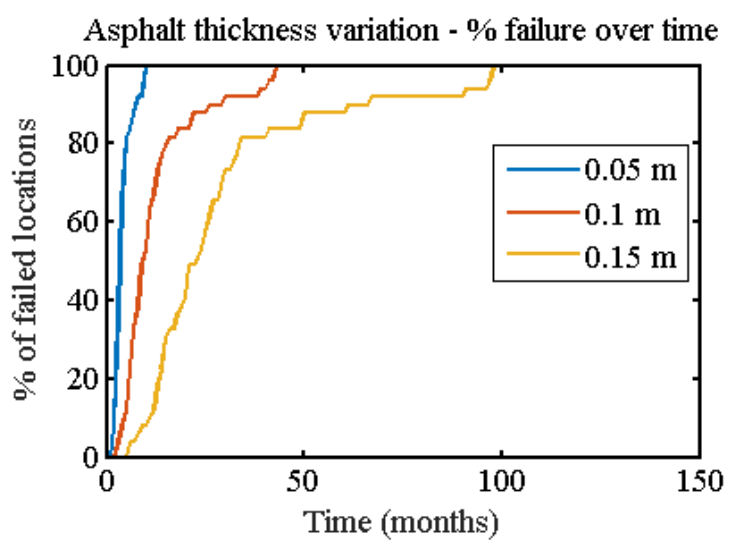

Figure 61: Asphalt thickness variation - \% of failed locations over time

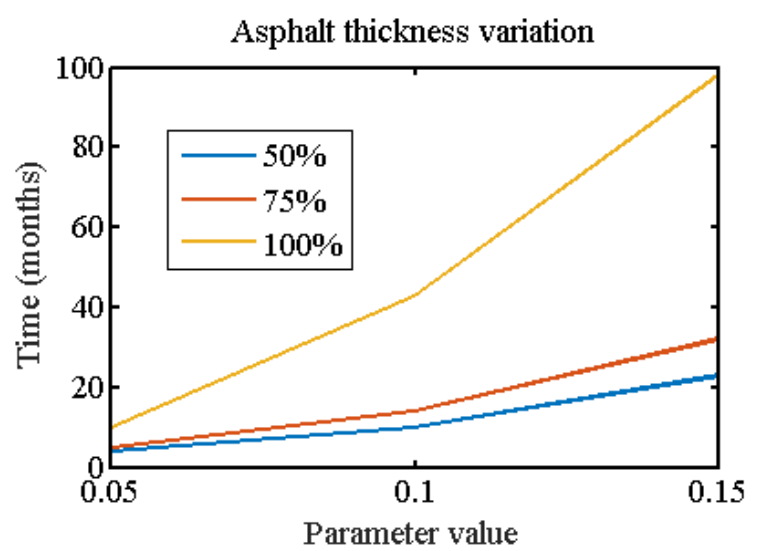

Figure 62: Asphalt thickness variation

\section{Concrete elastic modulus}

Something slightly different is shown in the case of the concrete elastic modulus. Varying the value of the concrete elastic modulus does not significantly affect the progression of the failure along time. When the value is $37500 \mathrm{MPa}$ the time required in general for the same amount of locations to fail is reduced slightly, as the concrete is stiffer. In the region of $12500 \mathrm{MPa}$ and $25000 \mathrm{MPa}$ it is shown that for $50 \%$ of locations to fail less time is required for the pavement with the higher concrete elastic modulus (25000 MPa), but on the other hand, when reaching $100 \%$ of failure it is shown that the pavement with the $12500 \mathrm{MPa}$ concrete elastic modulus reaches it first. This is a change of behavior that could also be explained by the parametric analysis in Chapter 2. It was shown that this parameter 
has a high dependence on the dominance of bottom-up or top-down cracking at each location, and therefore it could alter the behavior of the whole-life model.

Table 22: Time in months required for specific \% of failure with concrete modulus variation

\begin{tabular}{|c|c|c|c|}
\hline Concrete Modulus (MPa) & $\mathbf{1 2 5 0 0}$ & $\mathbf{2 5 0 0 0}$ & $\mathbf{3 7 5 0 0}$ \\
\hline Factor & 0.5 & 1 & 1.5 \\
\hline $50 \%$ & 12 & 10 & 9 \\
\hline $75 \%$ & 15 & 14 & 11 \\
\hline $100 \%$ & 38 & 43 & 35 \\
\hline
\end{tabular}

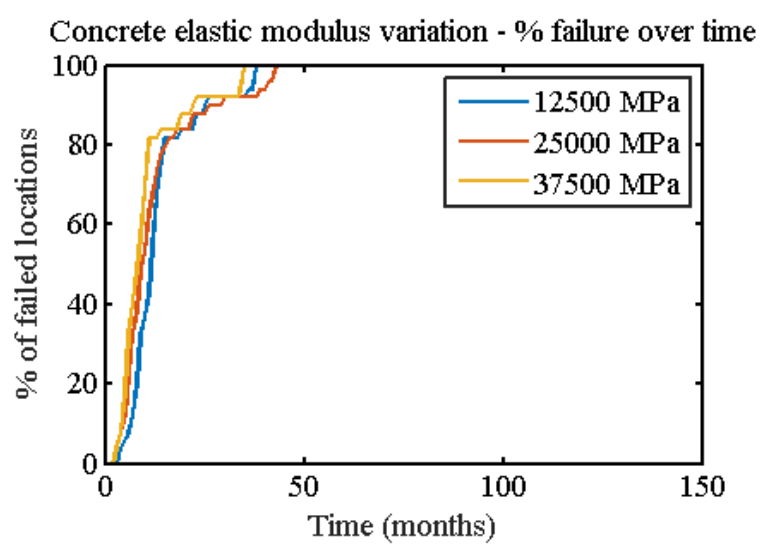

Figure 63: Concrete elastic modulus variation - \% of failed locations over time

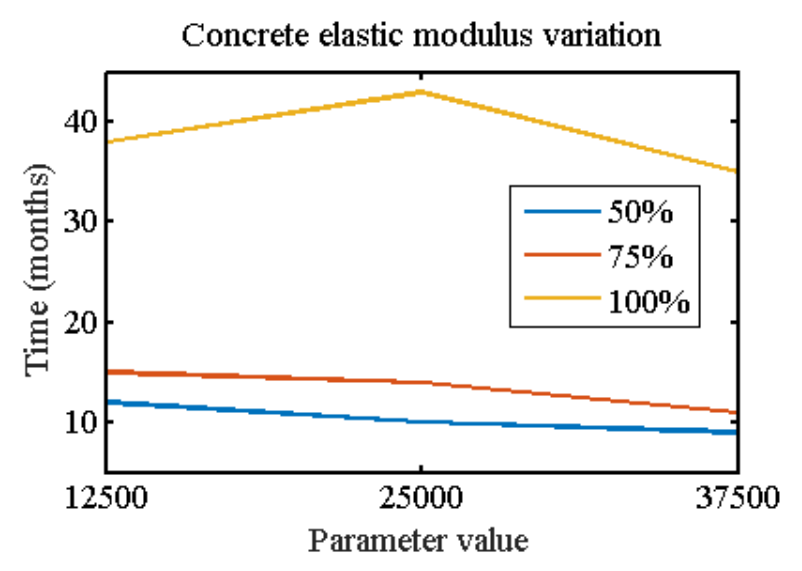

Figure 64: Concrete elastic modulus variation

\section{Concrete thickness}

In the case of the concrete thickness parameter it is shown in Figure 65 that increasing the concrete thickness from $0.1 \mathrm{~m}$ would highly affect the prediction and delay the cumulative failure of locations. However, increasing it further from $0.2 \mathrm{~m}$ to $0.3 \mathrm{~m}$ does not alter the prediction. In regards to time required for specific \% of failure to occur it is seen that in all three cases $(0.1 \mathrm{~m}, 0.2 \mathrm{~m}$ and $0.3 \mathrm{~m})$ the same amount of time is required for $50 \%$ of locations to crack. The fact that increasing the concrete thickness further than $0.1 \mathrm{~m}$ increases the time required for the locations to develop reflective cracking is shown also in Figure 66 by the line representing $100 \%$ for cases $0.2 \mathrm{~m}$ and $0.3 \mathrm{~m}$. Regarding them it is seen that for $100 \%$ failure to be reached a greater amount of time is needed compared to the $0.1 \mathrm{~m}$ case. 
Table 23: Time in months required for specific \% of failure with concrete thickness variation

\begin{tabular}{|c|c|c|c|} 
Concrete thickness (m) & \multicolumn{0}{c}{$\mathbf{1}$} & $\mathbf{0 . 2}$ & $\mathbf{0 . 3}$ \\
\hline Factor & 0.5 & 1 & 1.5 \\
\hline $50 \%$ & 10 & 10 & 10 \\
\hline $75 \%$ & 11 & 14 & 14 \\
\hline $100 \%$ & 20 & 43 & 41 \\
\hline
\end{tabular}

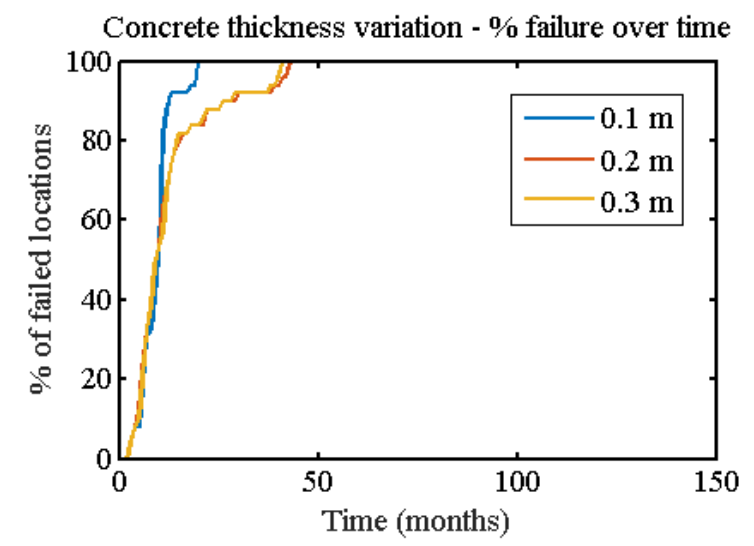

Figure 65: Concrete thickness variation - \% of failed locations over time

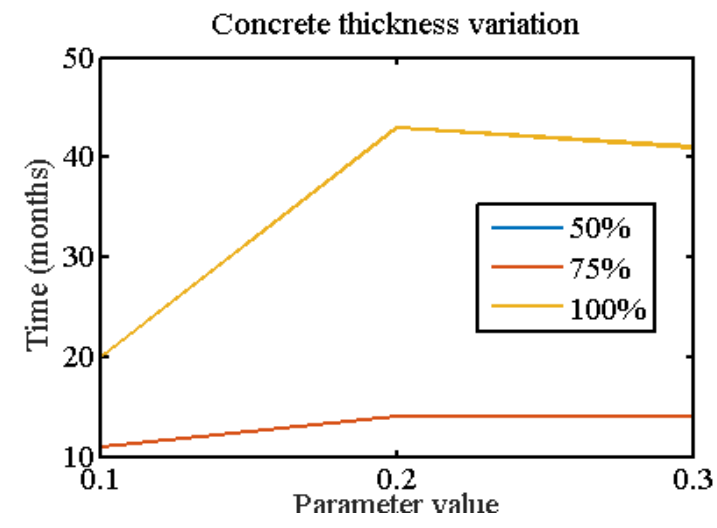

Figure 66: Concrete thickness variation

\section{Subbase elastic modulus}

Varying the subbase elastic modulus shows that for the lower elastic modulus subbase (100 MPa) the same amount of locations fails sooner than regarding the other two cases (200 MPa and $300 \mathrm{MPa}$ ) with increased values of subbase modulus. The difference becomes more obvious in Figure 68 by looking at the line representing the $100 \%$ of failure where from case $100 \mathrm{MPa}$ to $200 \mathrm{MPa}$ there is an increase in the required time for failure.

Table 24: Time in months required for specific \% of failure with subbase modulus variation

\begin{tabular}{|c|c|c|c|} 
Subbase Modulus (MPa) & $\mathbf{1 0 0}$ & $\mathbf{2 0 0}$ & $\mathbf{3 0 0}$ \\
\hline Factor & 0.5 & 1 & 1.5 \\
\hline $50 \%$ & 9 & 10 & 10 \\
\hline $75 \%$ & 13 & 14 & 15 \\
\hline $100 \%$ & 36 & 43 & 44 \\
\hline
\end{tabular}




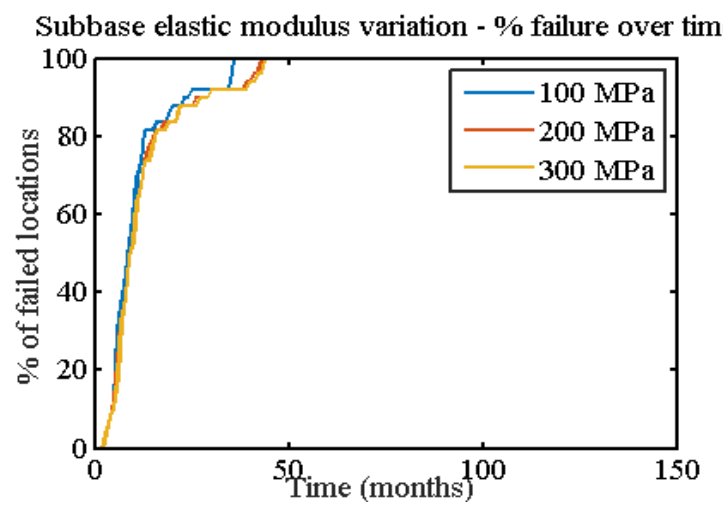

Figure 67: Subbase elastic modulus variation - \% of failed locations over time

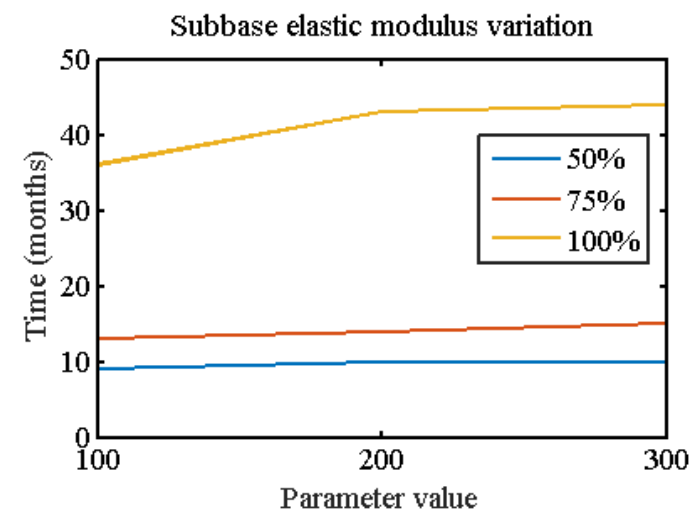

Figure 68: Subbase elastic modulus variation

\section{Subbase thickness}

It is seen from the following figures that the variation of the subbase thickness parameter does not affect the outcome of the predictions significantly. The difference of the case of $0.125 \mathrm{~m}$ subbase thickness with the other two cases $(0.25 \mathrm{~m}$. and $0.375 \mathrm{~m}$.) lies in that the final $10 \%$ of locations fails a few months quicker.

Table 25: Time in months required for specific \% of failure with subbase thickness variation

\begin{tabular}{|c|c|c|c|}
\hline Subbase thickness (m) & $\mathbf{0 . 1 2 5}$ & $\mathbf{0 . 2 5}$ & $\mathbf{0 . 3 7 5}$ \\
\hline Factor & 0.5 & 1 & 1.5 \\
\hline $50 \%$ & 10 & 10 & 10 \\
\hline $75 \%$ & 14 & 14 & 15 \\
\hline $100 \%$ & 40 & 43 & 44 \\
\hline
\end{tabular}

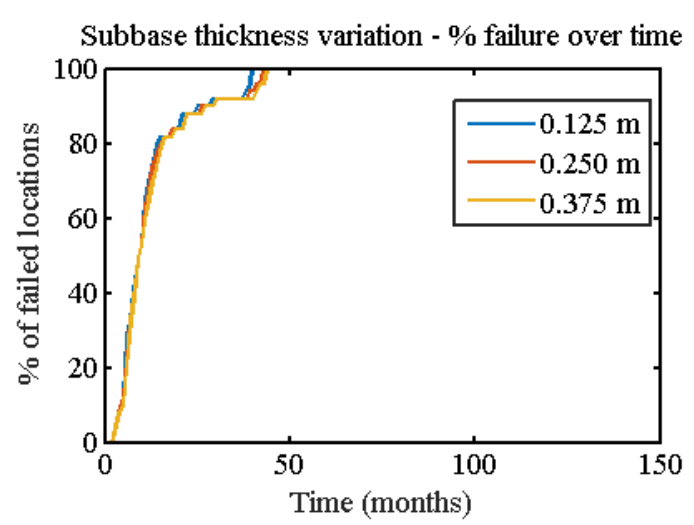

Figure 69: Subbase thickness variation - \% of failed locations over time

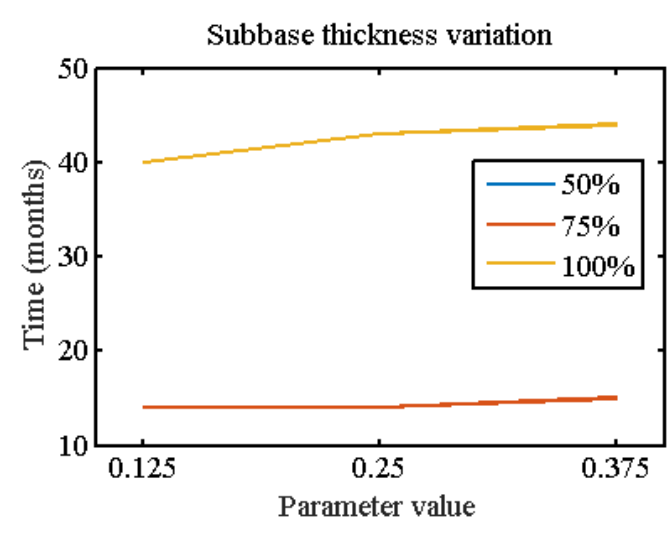

Figure 70: Subbase thickness variation 


\section{Subgrade elastic modulus}

When the subgrade elastic modulus is increased then as it is seen from Figure 71 failure progresses sooner. This is noticed in Figure 72 as well, where, it is shown that increasing the subgrade modulus value (on the $\mathrm{x}$ axis) reduces the amount of time required for $100 \%$ failure. In general, that was also seen in Chapter 2, in the parametric analysis without the vehicle dynamic loading. It was seen that increasing the value of the parameter would decrease the number of wheel loads required to failure, meaning a quicker failure.

\begin{tabular}{|c|c|c|c|}
$\begin{array}{c}\text { Table 26: Time in months required for specific \% of failure with subgrade } \\
\text { Subgrade Modulus } \\
\text { (MPa) }\end{array}$ & $\mathbf{5 0}$ & $\mathbf{1 0 0}$ & $\mathbf{1 5 0}$ \\
\hline Factor & 0.5 & 1 & 1.5 \\
\hline $50 \%$ & 11 & 10 & 9 \\
$75 \%$ & 17 & 14 & 12 \\
\hline $100 \%$ & 49 & 43 & 36 \\
\hline
\end{tabular}

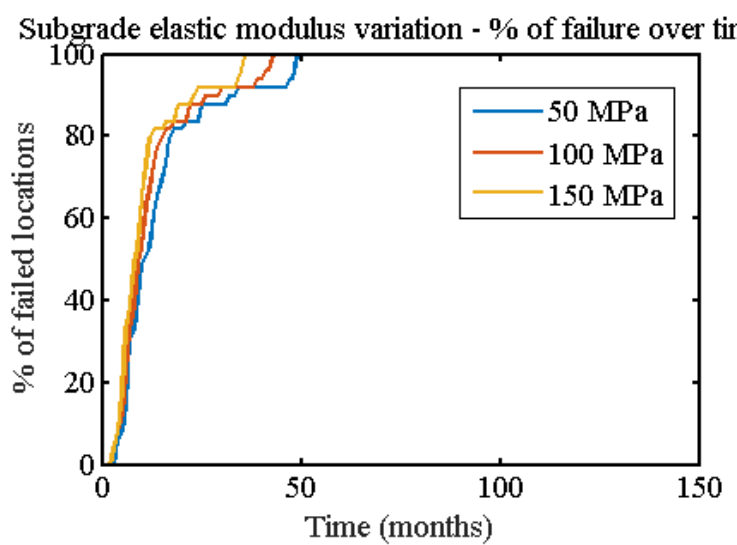

Figure 71: Subgrade elastic modulus variation - \% of failed locations over time

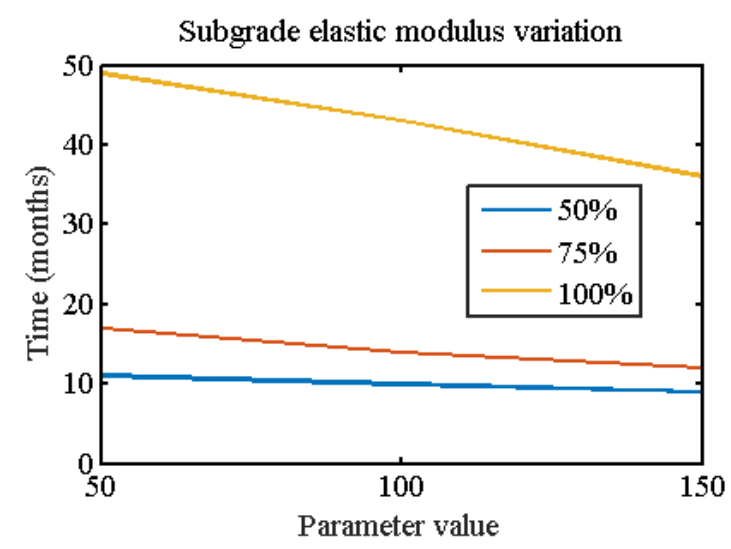

Figure 72: Subgrade elastic modulus variation 


\subsection{Summary}

After integrating all the models and mechanisms in a whole-life long-term composite pavement model, the dependence of the predictions from this model were investigated in order to identify their importance. First of all, the investigation of the appropriate increments was required for the basic time elements of the model. After conducting a convergence study the weekly time increment was selected for the new crack development and elastic modulus update, and the month time element for the IRI update on which the vehicle models depend on.

Also, a parametric analysis was conducted for the whole model this time, similar to the one presented in the 2nd chapter. It was found that regarding the asphalt and concrete layer property parameters the time required for $100 \%$ of failure to be reached is increased non-linearly compared to the time required for $50 \%$ and $75 \%$ of failure, showing a delay in the appearance of reflective cracks in the final locations. It was concluded from this analysis that the major parameters affecting the results were the asphalt elastic modulus and the asphalt thickness, while parameters such as the concrete elastic modulus showed unique results. For example increasing the concrete elastic modulus from $12500 \mathrm{MPa}$ to $25000 \mathrm{MPa}$ would require more time for the pavement to reach $100 \%$ failure, however increasing it more to $37500 \mathrm{MPa}$ would require less time. 


\section{Validation \& Results}

\subsection{Long-Term Pavement Performance database}

An important objective of this project is to validate the model using data from the Long-Term Pavement Performance (LTPP) Infopave database by comparing predictions to actual field measurements. The LTPP database includes monitored pavement performance data from selected pavement sites in North America. These sites belong to two categories: the General Pavement Studies (GPS) and the Specific Pavement Studies (SPS). The difference between the two catergories is that at the initiation of the LTPP program, the GPS sections were existing pavement sections, whereas the SPS sections were especially constructed pavement sections with controlled experimental factors.

The collected data which is of importance to the verification of the model includes the structural data of the pavement sections (layers, materials), the monitoring data which includes information about distresses, deflections and the profile of the pavement sections, the maintenance and rehabilitation actions on these sections, the climatic and traffic data. Data such as material properties and traffic can be used as input to the model and the distresses of the pavement sections can be compared to the actual measured distresses of the pavement as output predictions.

The selected pavement sections for use in the verification belong to the Specific Pavement Studies (SPS) category. Specifically, they belong to SPS 6 which represent the Rehabilitation of Jointed Portland Cement Concrete (JPCC) Pavements. Each SPS category consists of 8 experimental sections: 601 is the unrehabilitated control section, 602 and 605 have had no overlay, 603, 604, 606, 607 have had 4-inch $(101.6 \mathrm{~mm})$ asphalt overlays and 608 an 8-inch overlay (203.2 $\mathrm{mm})$. So only the overlaid sections are selected for the verification of the model.

Another reason for the selection of SPS pavement sections is that they were included in a National Pooled Fund study for collection of high quality traffic data of volumes, classifications and weights (Walker and Cebon, 2011). The states in SPS 6 with greatest amount of available traffic data were Illinois, Indiana, Pennsylvania and Tennessee. After thorough research of the data availability in LTPP 6 states were decided to be used for this study: Alabama (1), California (6), Illinois (17), Iowa (19), Pennsylvania (42), Tennessee (47). 
It should be noted that on section 604 along with the asphalt overlay, the maintenance technique of saw and seal also took place. This is a technique where cracks are created and sealed on purpose in the asphalt layer at positions directly on top of existing cracks in underlying layers. This aims to predetermine the areas where the cracks will develop in the asphalt layers as well as to ensure their condition. In addition to that, 607 and 608 have also gone through a worth-mentioning rehabilitation technique which is that of crack and seat. This is a technique where, before the asphalt overlay is placed hairline cracks are created in the rigid layer, to make it more flexible so it can accommodate movements and it is also seated into the subbase so it can be more stable. These two techniques, saw and seal and crack and seat, aim generally to the prevention of the harmful results of reflective cracking. Therefore, for validation sections 603 and 606 have been used mainly due to the similarity of their characteristics.

The input required for the whole model to work are:

- Asphalt layer thickness

- Concrete layer thickness

- Subbase thickness

- Length of the slab of the concrete layer

- Daily temperatures

- Elastic modulus of asphalt layer

- Elastic modulus of concrete layer

- Elastic modulus of subbase layer

- Elastic modulus of subgrade layer

- Speed distribution of traffic

- Traffic data (daily, weekly, monthly, yearly) categorized in a specific weight group.

- Crack Shear Modulus

- International Roughness Index (IRI)

- Transverse Cracking Distress

For most of them the procedure is straightforward to find this data. For some it is more complicated and the data in the Long-Term Pavement Performance database needs extra analysis and processing. 


\subsubsection{Transverse cracking distress}

The distresses that have been measured and are of interest are the number of transverse cracks on each pavement section. From these, the number of reflective cracks can be determined by comparing the distress maps before the asphalt overlay took place, with the distress maps years after the asphalt overlay to see if the new cracks match the ones that existed before the asphalt overlay. In Figures 73(a-f) the development of transverse cracking is shown for each of the states used in this study and specifically for sections 603 and 606, due to their suitability by both having received 4-inch asphalt overlays and not any anti-reflective cracking measures. In the database, the number of transverse cracks is given in three categories depending on their severity high, medium and low. These have been summed up as a total number of transverse cracks and divided by the total length of each pavement section which is approximately $150 \mathrm{~m}$. to find the number of transverse cracks per metre.

So, on the vertical axis the number of transverse cracks per metre is shown and on the horizontal the year which the measurement survey was performed. The first year on each figure for each state is when the asphalt overlay was placed. A general notice is that in all the states an increase in the number of transverse cracks is shown in the first years of measurements, except from certain cases where it remains stable with an increase only in the final year measurements, like the cases of Illinois and Pennsylvania. This would also depend on the number of transverse cracks that existed before the overlay on the concrete layer.

The development of the number of transverse cracks is different between each state. For some of them like Illinois and Pennsylvania in the years after the first measurement there is little or no increase in the value of number of transverse cracks per m. In states such as California though, as well as Iowa there is a visible increase in the number of transverse cracks per m. from the first years. That has to do with the different structure each section has as well with the difference in the pre-existing concrete layers underneath in the amount and severity of the cracking. A difference that can be noted between them is that regarding Illinois and Pennsylvania, the underlying distance between cracks or joints is quite large over $7 \mathrm{~m}$., whereas for California and Iowa this crack spacing is $3 \mathrm{~m}$. and $5 \mathrm{~m}$. respectively. This data can be seen in Table 27 where all the basic data of the pavement structures are shown, presented later on. 

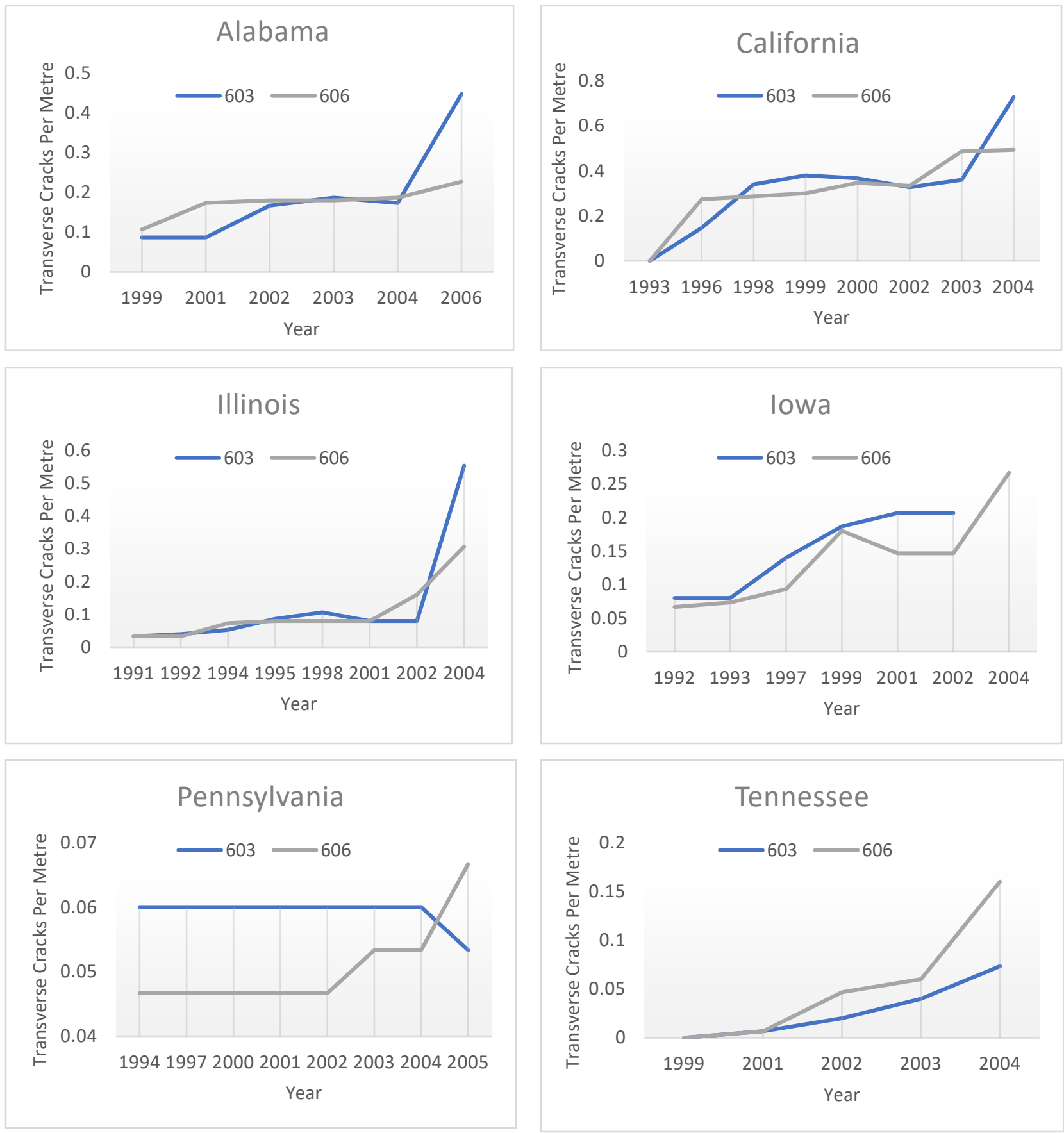

Figures 73(a-f): Transverse cracks per metre along time for 6 states.

(a: Alabama, b: California, c: Illinois, d: Iowa, e: Pennsylvania, f: Tennessee)

For the estimation of the total amount of transverse cracks another adjustment was also made. Due to the extent of the damage on the surface of the asphalt which becomes very severe years after the construction of the overlay, the number of transverse cracks is no longer given separately in the 
database, but it is incorporated in other more general types of distresses like block or fatigue cracking which are measured by the amount of area that is cracked. With the help of the distress maps these lengths were located and their lengths were included in the total number of transverse cracks.

So, the graphs in Figures 73 confirm the existence of transverse cracking on these 6 sections and subsequently of reflective transverse cracking. It is shown that for each state, a different amount of years after the LTPP section initiation is required, to develop transverse cracking.

It should be mentioned that reflective crack measurements are not included in the database. They can be estimated with the help of the transverse crack data measurements along with visual help from the distress survey maps. A comparison can be made between the distress map before the asphalt overlay (1990) and the distress map the years after the asphalt overlay. The transverse cracks that develop in the same positions as previous cracks or joints before the overlay (allowance up to $1 \mathrm{~m}$ distance with exceptions) can be considered as reflected transverse cracks.

In order to see if the cracks have all reflected after the placement of the asphalt overlay a comparison was made between the new transverse cracks that developed along the years after the asphalt overlay and the number of transverse cracks that existed before the asphalt overlay. This comparison is made in the form of a percentage, so when 1 is reached it would mean that $100 \%$ of the pre-existing cracks reflected. This is shown in the following Figure 74. On the horizontal axis is the months after the overlay and on the vertical axis is the percentage of:

$$
\text { Ratio }=\frac{\text { No.of transverse cracks after asphalt overlay }}{\text { No.of transverse cracks before asphalt overlay }}
$$

Each point shown on the figure refers to a specific measurement survey of the number of transverse cracks at that time (number of months after the overlay took place). If the percentage reaches the value 1 it can be assumed that all the pre-existing transverse cracks reflected. This percentage is over 1 for all the states in the study as can be seen from figure. In two cases especially though (Alabama and California) these ratios exceed the value 1 which means that the new pavement develops transverse cracking in more locations than before the asphalt layer placement. Also, it is important to notice that the time required for the ratio to reach 1 for each state differs significantly between each case. 


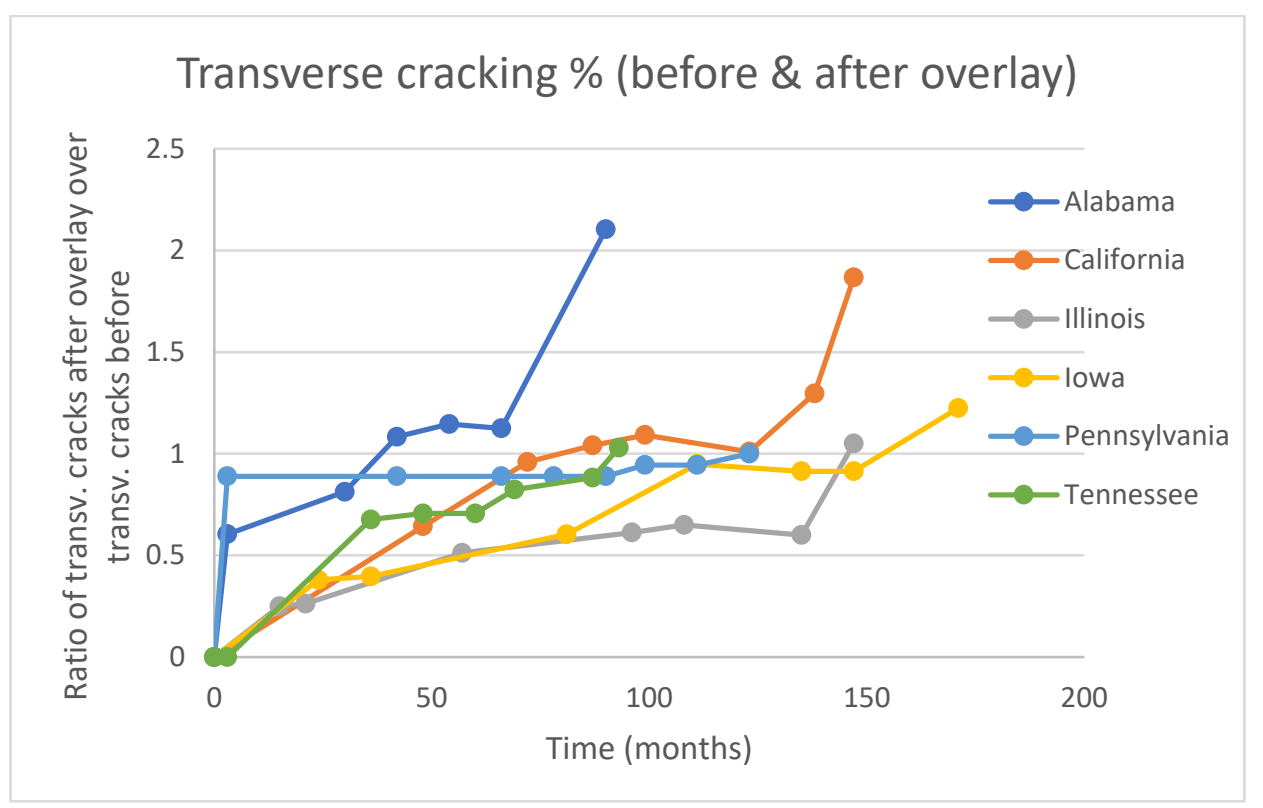

Figure 74: Ratio of transverse cracks before and after overlay.

\section{Recognising reflective cracks from distress maps}

The development of reflective cracking can be visualized better with the help of the distress maps from the LTPP database. Two examples are given below.

1st example:

The first is an example of section 603 from the state of California and the locations $90 \mathrm{~m}$ to $120 \mathrm{~m}$. The progress of reflective cracking on the surface of the pavement can be seen from the following distress maps taken on different dates in the pavements life shown in Figure 75 . The specific pavement section was constructed originally in November 1973. It was included in the LTPP study in January 1987 and the asphalt overlay was placed in May 1992. The first distress map shows the state of cracking on the surface of the pavement on 21/02/1992 which is before the asphalt overlay. The asphalt layer was placed as a rehabilitation action in May of 1992. Below the first distress map, distress maps from years 1993, 1996 and 1999 (1 year, 4 years and 7 years after the asphalt overlay) are also shown.

With the yellow dashed lines are presented the low severity cracks (transverse or longitudinal), with the green dashed lines the medium severity cracks and with the red dashed lines are represented the high severity cracks. The black dotted lines represent the joints between the slabs of the concrete 
layer. All of these cracks or joints before the asphalt overlay in February of 1992 represent the potential locations where reflective cracking may appear on the surface of the asphalt overlay along the years.

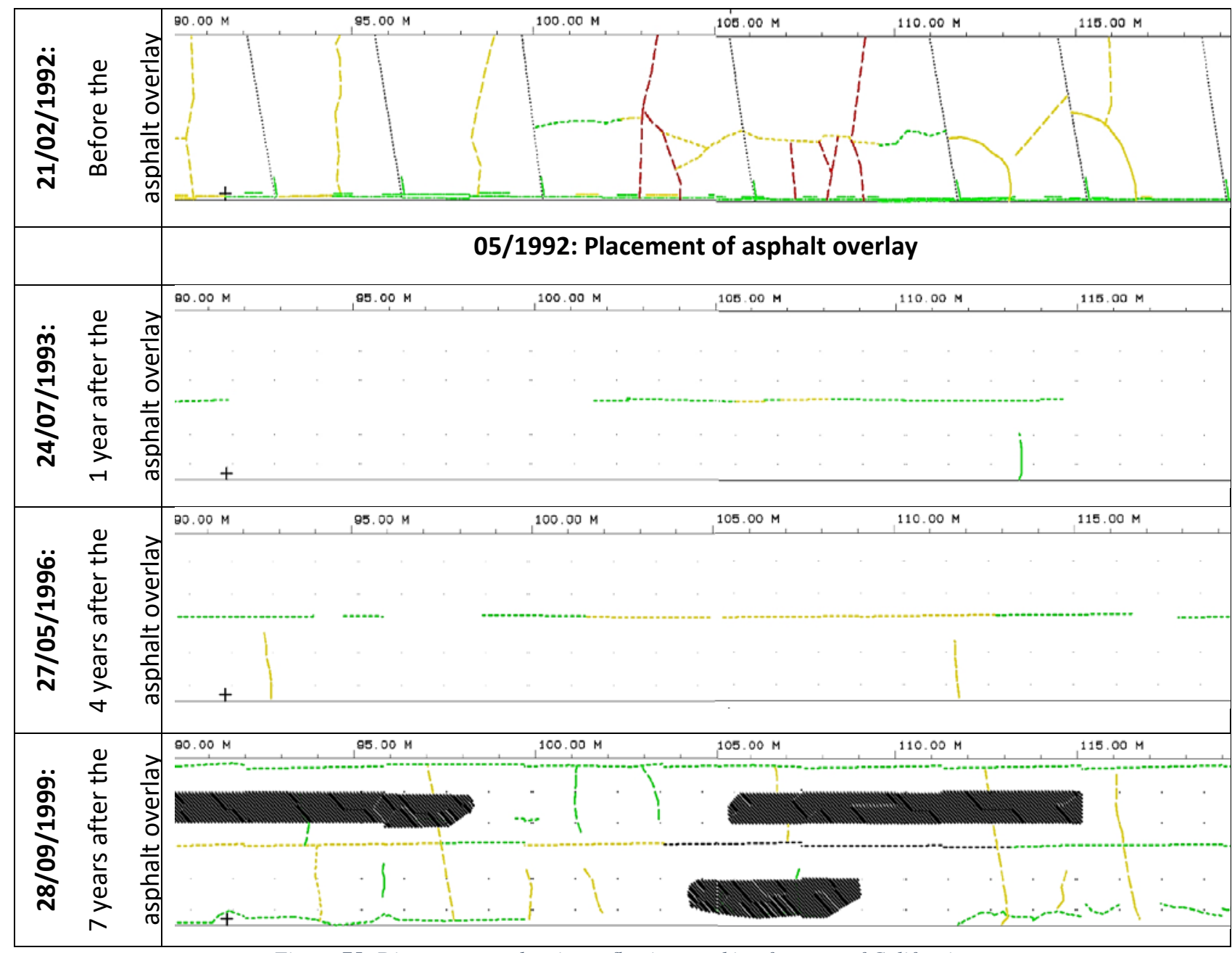

Figure 75: Distress maps showing reflective cracking for state of California.

It can be seen clearly that with time, reflective cracks develop on the surface of the asphalt layer at similar locations as in the original pavement (before the overlay). In the distress map of 1993 it can be seen that the first transverse crack is starting to reflect on the surface at the location of $114 \mathrm{~m}$. In the following distress map of 1996 it is shown that another crack is starting to reflect around the location of 92m. In the final distress map of 1999 it can be seen that a lot of the previously existing cracks or joints have reflected through the asphalt thickness. Except the clearly defined cracks there 
are black hatched areas which reveal the presence of fatigue cracking and cover the transverse reflective cracks that had developed.

Unfortunately, after the year 1999 there are not any available distress maps of this type in the database, but only photos of the pavement surface. Therefore, they cannot be used for this comparison purpose. This example can show the kind of data and information that are included in the LTPP database. It shows exactly the procedure that should be followed in order to identify the appearance of reflective cracks with this data availability.

$2^{\text {nd }}$ example:

The second example is taken from the state of Illinois section 603 and the locations $90 \mathrm{~m}$ to $120 \mathrm{~m}$. This pavement section was originally built in June 1964 and inserted in the LTPP study in January of 1987. The asphalt overlay was placed in June of 1990.

The first distress map in Figure 76 shows the condition of the pavement surface one month before the placement of the asphalt overlay in May of 1990. In June of 1990 the asphalt overlay is placed. The first distress map after the asphalt overlay placement is 2 years after, in 1992, and it shows reflective cracks at the location of 93m where a joint previously existed and at the location of 110m where a high severity transverse crack existed.

Distress maps following show the development of reflective cracks at the locations with pre-existing cracks in the underlying layer like for example at location of $105 \mathrm{~m}$ and $115 \mathrm{~m}$. In this example it is also worth noticing that some transverse cracks can reflect not only at the same exact locations as the pre-existing ones but also at locations at a distance around $1 \mathrm{~m}$ or $2 \mathrm{~m}$ from the original ones. This is seen at the location $103 \mathrm{~m}$. 


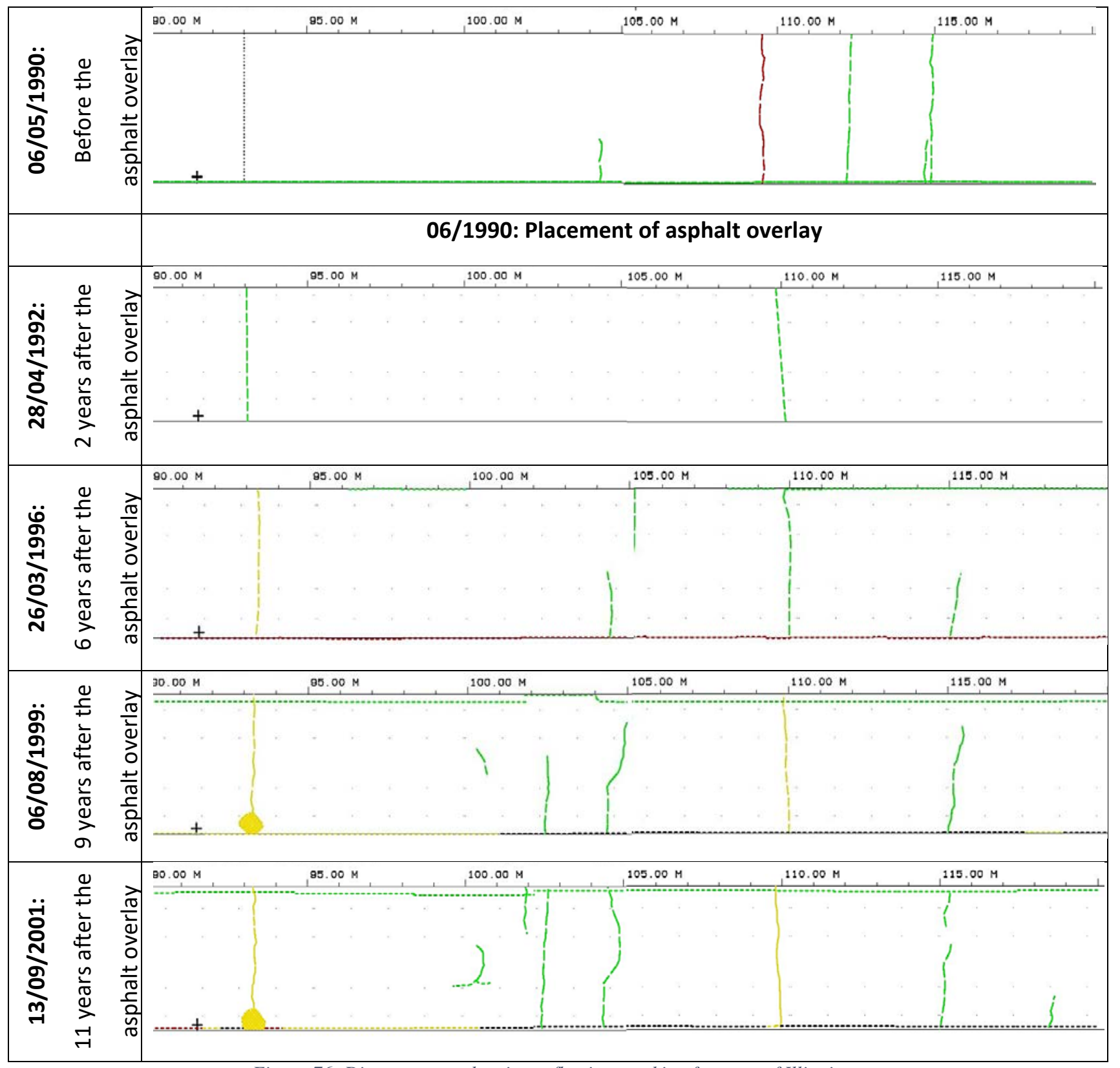

Figure 76: Distress maps showing reflective cracking for state of Illinois.

\subsubsection{Layer properties}

\subsubsection{Structure}

It is quite important to use the correct input data in the program. One of the most significant parts of information regards the structure of the pavements. Specifically, that means the type of layers that consist the pavement, the layer thicknesses, the pavement section length, the length of the slabs or 
the average length between two consecutive cracks in the underlying layer. The table that follows (Table 27) shows some of the data regarding the structure of the pavement sections, used as input.

The information included in this table regard the 6 states that are studied and regard the climate, the thicknesses of the asphalt layer, the concrete layer that pre-existed, the subbase layer and the length of the underlying slab (or crack to crack distance).

Table 27: Basic structural input data from 6 states.

\begin{tabular}{|c|c|c|c|c|c|c|}
\hline States & $\mathbf{1}$ - Alabama & $\mathbf{6}$ - California & $\mathbf{1 7}$ - Illinois & $\mathbf{1 9}$ - lowa & $\mathbf{4 2}$ - Pennsylv. & $\begin{array}{c}\mathbf{4 7}- \\
\text { Tennessee }\end{array}$ \\
\hline $\begin{array}{c}\text { Climatic } \\
\text { Zone }\end{array}$ & $\begin{array}{c}\text { Wet/ } \\
\text { Non-Freeze }\end{array}$ & $\begin{array}{c}\text { Wet/ } \\
\text { Non-Freeze }\end{array}$ & Wet/Freeze & Wet/Freeze & Wet/Freeze & $\begin{array}{c}\text { Wet/ } \\
\text { Non-Freeze }\end{array}$ \\
\hline $\begin{array}{c}\text { Asphalt } \\
\text { thickness }\end{array}$ & 0.1 & 0.12 & 0.1 & 0.1 & 0.1 & 0.1 \\
\hline $\begin{array}{c}\text { Concrete } \\
\text { thickness }\end{array}$ & 0.26 & 0.21 & 0.25 & 0.25 & 0.25 & 0.23 \\
\hline $\begin{array}{c}\text { Subbase } \\
\text { thickness }\end{array}$ & 0.15 & 0.11 & 0.18 & 0.18 & 0.25 & 0.19 \\
\hline Slablength & 6 & 3 & 7 & 5 & 15 & 8 \\
\hline
\end{tabular}

The climate zones that the different chosen states belong to are Wet and Non-Freeze as well as Wet and Freeze covering two of the possible categories. In the SPS 6 category which is of interest for this research these are the two main categories that can be covered. Factors such as the asphalt thickness, the concrete thickness and the subbase thickness are important in the program and can influence and determine the result. The slab length selected for each state is important because it reveals how many are the potential locations that can develop reflective cracking on the surface of the asphalt overlay. It is selected from data included in the database that cover the length of the slab but also from investigating the distress maps before the placement of asphalt. In most instances transverse cracks have appeared in between the joints therefore increasing the possible locations of reflective cracking. An average length between joints or transverse cracks is therefore selected for each state. For example, the distress map in Figure 75 which refers to California shows that an average length of slab of $3 \mathrm{~m}$. could be selected. 


\subsubsection{Layer elastic moduli}

The elastic moduli of the materials the pavement consists of, are inserted in the program to characterise the stiffness of the layers and their structural importance. This data is found in the Long -Term Pavement Performance database, and it originates from backcalculated data of Falling Weight Deflectometer (FWD) measurements that took place for this reason. In order to gather the necessary data for input in the program, processing of the original data was required. First, based on the shape of the basin, only measurements that refer to a so-called "typical" basin in the FWD measurements, which is consistent with the elastic layer theory, are used. Also, measurements for which the RootMean-Squared-Error computed for the individual deflection basin is greater than $3 \%$ are excluded from the calculations due to the high error.

Four different drop heights with different target loads are used in the original measurements and the results are also presented in that way. The measurements from the second drop height with a target load of $40 \mathrm{kN}$ are the ones used because this target load is similar to the load a vehicle wheel applies. Except from this differentiation of the different weight loads the results are also separated in those that refer to deflection measurement in the outer wheel path and those from mid-lane path. The elastic moduli inserted in the program and used in further calculations are based on the outer wheel path calculations to take into account the effect of the vehicle loading.

Measurement surveys took place once every few years not necessarily on the same date or year for all the sections. The most valuable data for the research is the elastic moduli on the year that is the closest after the placement of the asphalt overlay. The deflection measurements, and as a consequence the backcalculated elastic moduli data, are taken at specific locations on a pavement section of 152 m., not always being at the same points for each measurement survey.

That is why in the following figure (Figure 77), what is shown is the elastic moduli along the length of the pavement, for the first year after the asphalt overlay. In the example of California, the first measurement was in 1992 and the elastic moduli values along the 152m. section of 603 do not vary much in comparison to the section 606 which show the variability that can appear in the measurements even of the same year but at different locations. This variability also depends on the severity of the cracking underneath the asphalt overlay in the previous rigid's composite pavement Jointed Plain Concrete layer. 


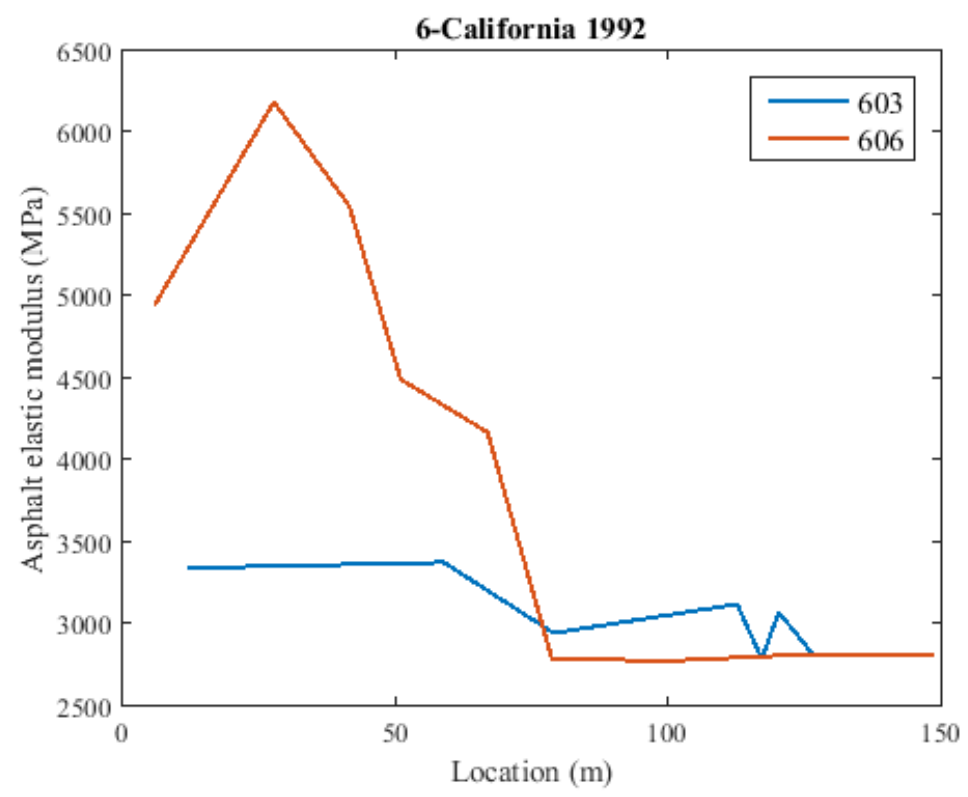

Figure 77: Asphalt elastic modulus on the year after the asphalt overlay for California.

The variations of the elastic moduli values along the pavement section, that are a result of one measurement survey, show the necessity of averaging the values of that specific pavement section over the whole length. These variations are most probably due to the different conditions during the measurement at each location as well as the different condition of the underlying layers. For example, these differences could be in the severity of the cracking of the concrete at certain locations compared to other locations (low, medium or high severity).

An adjustment was made to the data, which was to average the values of the moduli of all the locations of one specific pavement section for each measurement year. This was done to simplify calculation and insert one average value of elastic modulus in the program regarding each pavement layer which would be the same for all locations. So, in this version of the program no differentiation is made between the locations on the pavement section.

An example from a section of one state is given to show the procedure that was followed. In Figure 78 the average elastic modulus of the asphalt layer of all the locations ( $0 \mathrm{~m}$ to $152 \mathrm{~m}$ ) of sections 603 and 606 in the state of California are given for each measurement survey, from the first year after the overlay in 1992 up until the year of the final measurement in 2004. This figure reveals that because of the variation in the parameters at every FWD measurement, as well as the backcalculation 
procedures, it is normal for the elastic moduli values not to have a proper inclination along the years (decreasing values or increasing). Therefore, these backcalculated measurements can only be used as an indicator to which value should be used as an input to the program after the asphalt overlay.

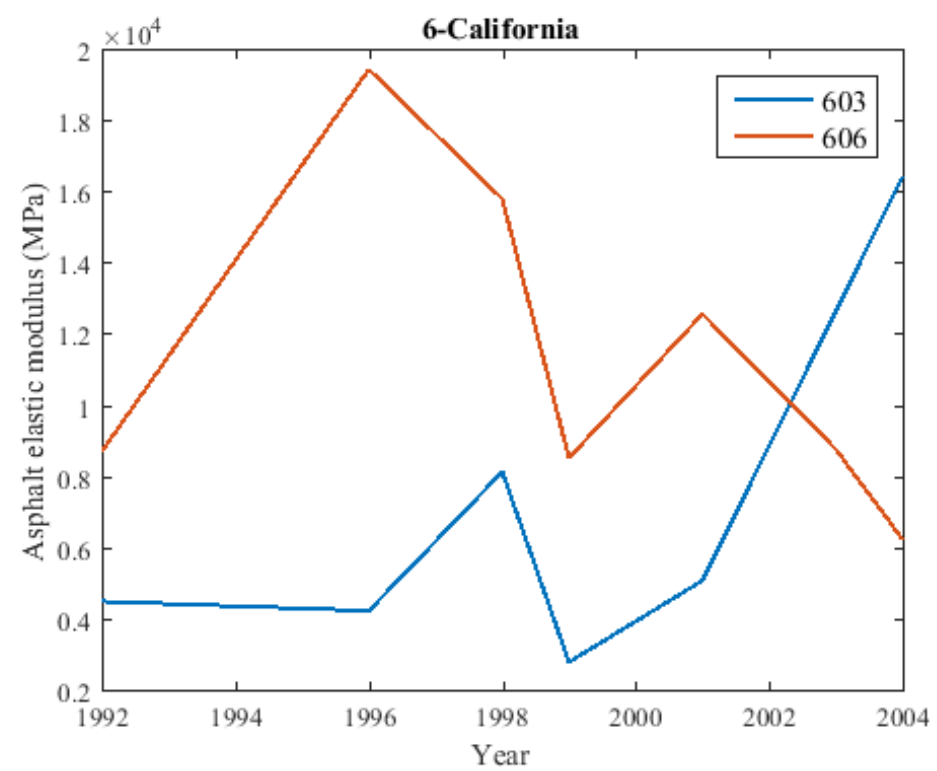

Figure 78: Asphalt elastic modulus along time for California.

The values that are used effectively in the program are those regarding the first measurement after the asphalt overlay in 1992.

Table 28: Average elastic moduli data for each state.

\begin{tabular}{|c|cccccc|}
\hline States & 1 - Alabama & 6 - California & 17 - Illinois & 19 - Iowa & 42 - Pennsylv. & 47 - Tenness. \\
\hline $\begin{array}{c}\text { Asphalt } \\
\text { modulus (MPa) }\end{array}$ & 6280 & 3570 & 12530 & 22400 & 3760 & 5480 \\
$\begin{array}{c}\text { Concrete } \\
\text { modulus (MPa) } \\
\text { Subbase }\end{array}$ & 50800 & 27870 & 46980 & 22980 & 22630 & 70 \\
$\begin{array}{c}\text { modulus (MPa) } \\
\text { Subgrade } \\
\text { modulus (MPa) }\end{array}$ & 230 & 80 & 70 & 65 & 1130 \\
\hline
\end{tabular}

In the above table (Table 28) are given the average elastic moduli in MPa that are used as input in the program. They are based on the outer wheel path calculations to take in account the effect of the vehicle loading. 
It is important to note here that, the exact material properties of the asphalt layer are not included for every pavement section in the database. For example, binder content, aggregate information could have an effect on the final result but at this point only a general elastic modulus is taken in account.

\subsubsection{Asphalt modulus temperature correction}

In Chapter 5, the procedure for the temperature correction of the asphalt elastic moduli is presented. The equation used for that requires a slope value which represents the slope in a trendline of $y=a x+b$ where a is the slope. To find that, the values of the asphalt elastic moduli are placed on an x-y graph, with the log of the elastic moduli on the y axis and the daily temperatures at the area of that pavement section on the $\mathrm{x}$ axis.

Depending on the aggregate properties and the characteristics of the binder, the slope of Eq. (7.2) is calculated. This varies usually between -0.013 to -0.032 . The slopes are negative due to the fact that the elastic moduli decrease while the temperatures increase. The FWD measurements used for the calculation of the slope refer to the mid-lane measurements and not the outer wheel path. That is done in order to eliminate the effect of the wheel loading and to consider just the effect of the temperature. The average slope selected for each state is shown in the following table.

Table 29: Slopes for elastic modulus temperature correction procedure.

\begin{tabular}{|c|c|c|c|c|c|c|}
\hline States & Alabama & California & Illinois & Iowa & Pennsylv. & Tennessee \\
\hline Slope & -0.0203 & -0.0291 & -0.0186 & -0.0185 & -0.0304 & -0.0171 \\
\hline
\end{tabular}

An example of the above procedure is given for section 603 of the state of California is given in Chapter 5, where an example case was presented (5.3 Application of model for pavement with baseline values).

\subsubsection{Traffic data}

In the LTPP database there is a range of data available for traffic on most of the pavement sections. According to the availability of the data, the data most suitable to the study is the daily axle data for each state which is divided according to a weight group data and vehicle class (DD_AX tables in LTPP). These tables consist of daily data of single axles, tandem, tridem and quad loading which are converted to individual loading to suit the needs of the program. According to the weight of the axles the data is divided in 40 different axle weight groups. For example, the first one includes the number 
of axles whose weight is in the range between 0-999 pounds $(0-4 \mathrm{kN})$ if they are single axles, 0-1999 pounds $(0-8.89 \mathrm{kN})$ if they are tandem axles and $0-2999$ pounds $(0-13.34 \mathrm{kN})$ in the cases of tridem or quad axles. The second one includes the single axles whose weight belong to 1000-1999 pounds $(4.44 \mathrm{kN}-8.89 \mathrm{kN})$ and it continues the same way for 40 groups.

To make the calculations less complicated the 40 groups were converted into 4 by averaging every 10 groups together and later converted into 4 individual wheel weight groups. Table 30 shows the 4 different wheel weight groups and their average total weight per vehicle.

Table 30: Wheel weight range and groups.

\begin{tabular}{|l|c|c|c|c|}
\hline \multirow{2}{*}{ Range (kg) } & 111 & 2335 & 4559 & 6783 \\
\cline { 2 - 5 } & 2112 & 4337 & 6561 & 8785 \\
\hline Weight (kg) & 1112 & 3336 & 5560 & 7784 \\
\hline
\end{tabular}

Therefore, the traffic data as well, is inserted in the program in 4 groups and not 40 to simplify the calculations but a simple conversion is made within each group regarding the amount of traffic that represent single, tandem, tridem and quad axles.

\subsubsection{IRI - Number of transverse cracks correlation}

This is done with the help of correlation equations from real data regarding the number of transverse cracks and the value of the International Roughness Index (IR) from each state (Alabama, California, Illinois, Iowa, Tennessee and Pennsylvania). IRI values in the database along time show an increase as the number of transverse cracks on the surface of the asphalt pavement increases. These relationships are shown in the following figures for each state (Figure 79 (a-f)).

These correlations are based on data for 5 pavement sections from each state (603 (blue), 604 (orange), 606 (grey), 607 (yellow), 608 (light blue)) which are shown with the different bullet colours on each figure. 

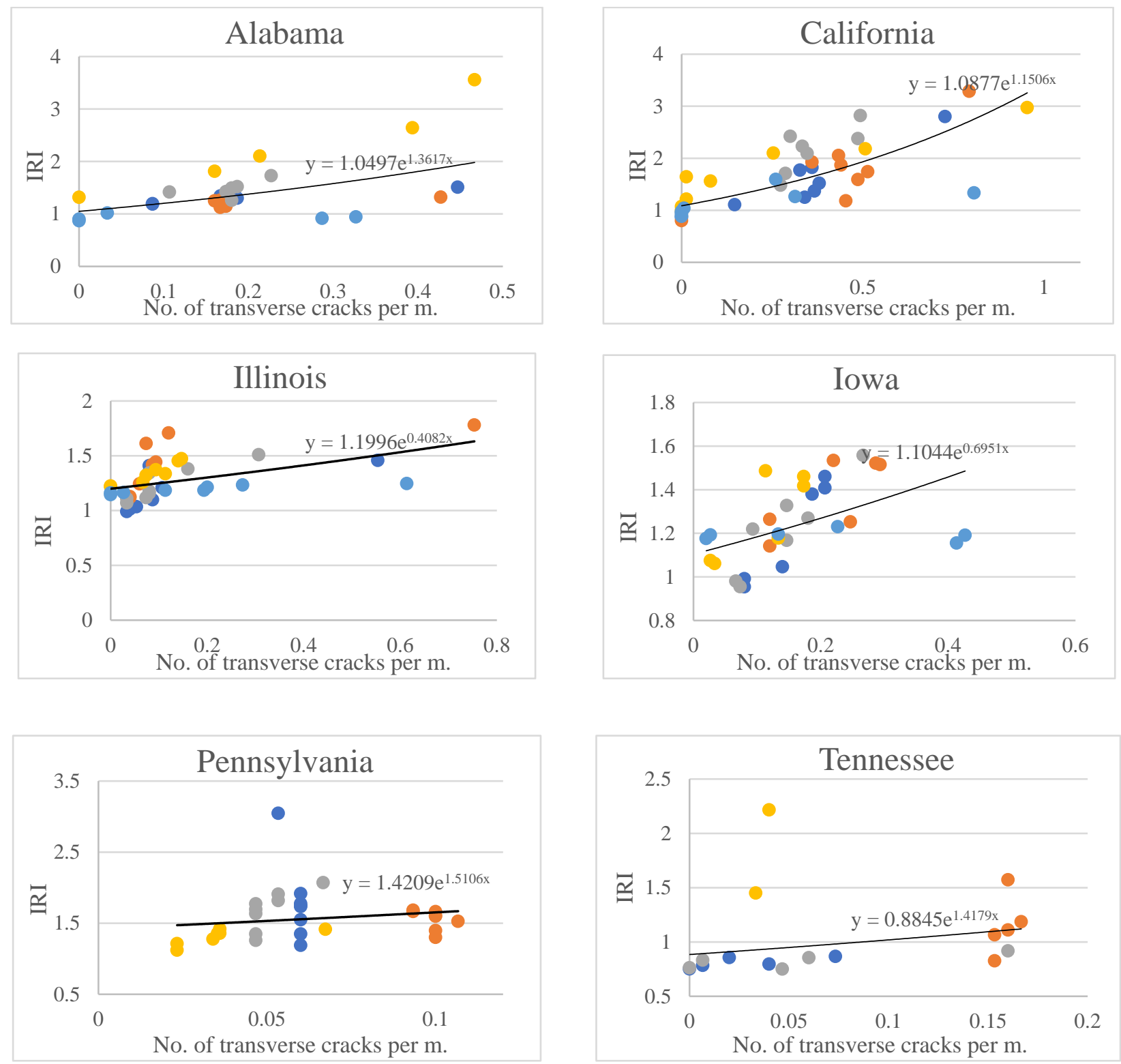

Figure 79(a-f): Correlation between IRI - No. of transverse cracks per m. (a: Alabama, b: California, c: Illinois, d: Iowa, e: Pennsylvania, f: Tennessee)

\subsection{Validation Simulations}

7.2.1 Examples of failure with reflective cracking at a single location

A 150m typical pavement section from a given state is examined and as was explained earlier in this chapter each pavement section which was previously a rigid pavement was built originally with different length of slabs. In some situations, before the asphalt overlay placement, transverse cracks 
had developed in between joints on the rigid pavement surface. These locations, where joints or transverse cracks exist, are the locations where potential reflective cracks can develop. To identify these locations standard distance between cracks or joints in the underlying layers has been selected (Table 27). These are also depicted for examples of pavement sections from different states in the idealised figures below where each potential location of reflective cracking is numbered on a $50 \mathrm{~m}$ part of the total pavement section.

1 - Alabama (a):

\begin{tabular}{|l|l|l|l|l|l|l|l|l|}
\hline \multicolumn{19}{|c|}{ Asphalt overlay } \\
\hline & 1 & 2 & 3 & 4 & 5 & 6 & $\begin{array}{l}7 \\
\text { Concrete lalver }\end{array}$ \\
\hline \hline
\end{tabular}

6 - California (b):

\begin{tabular}{|l|l|l|l|l|l|l|l|l|l|l|l|l|l|l|l|l|}
\hline & 1 & 2 & 3 & 4 & 5 & 6 & 7 & 8 & 9 & 10 & 11 & 12 & 13 & 14 & 15 & 16 \\
\hline
\end{tabular}

17 - Illinois (c):

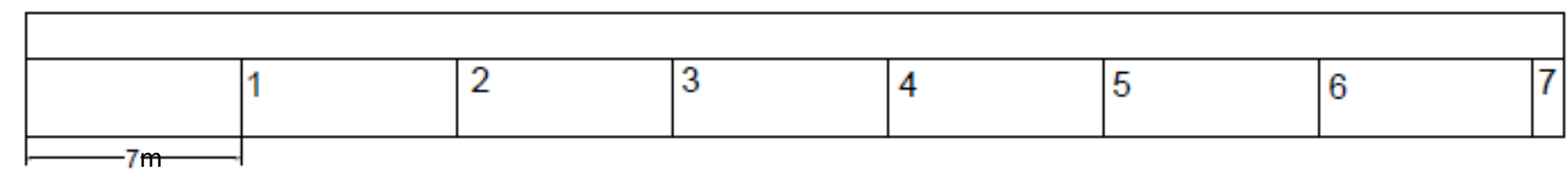

19 - Iowa (d):

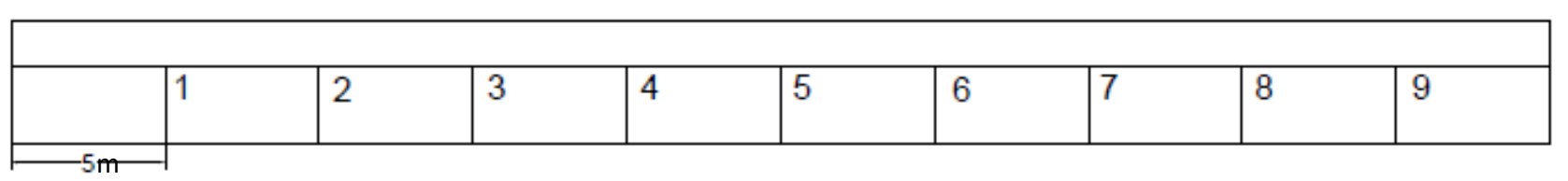

42 - Pennsylvania (e):

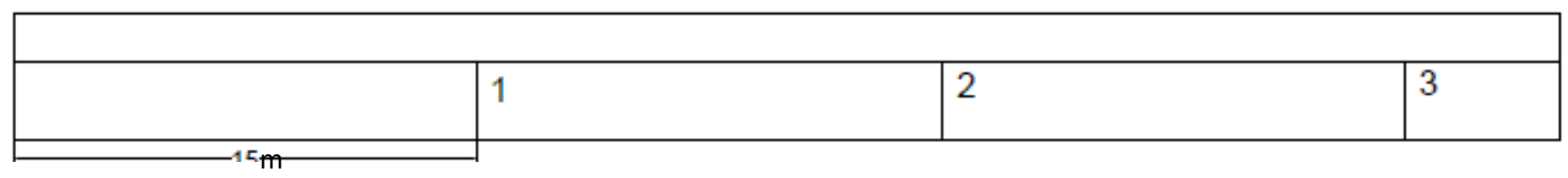

47 - Tennessee (f): 


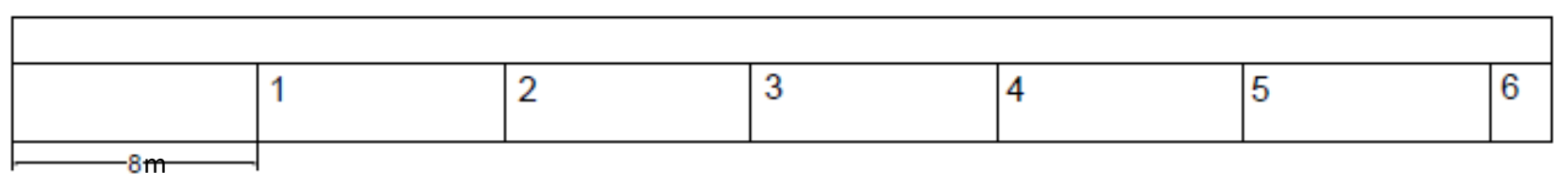

Figure 80(a-f): Idealised composite pavements from each state.

(a: Alabama, b: California, c: Illinois, d: Iowa, e: Pennsylvania, f: Tennessee)

Depending on the dynamic tyre forces calculated at each location, there is a different development of bottom-up and top-down cracking. In the following, examples of reflective cracking at certain locations of each pavement section as an output from the program are given. The following figures (Figure 81, a-f) show how the cracks progress from the top and from the bottom in randomly chosen locations. The examples that are given regard the 1st location and the 5th location as were seen in the figures above. On the y axis is the level of cracking in the asphalt layer in mm to which each crack from top to bottom develops over a certain number of wheel loads that traffic the pavement section which are shown on the $\mathrm{x}$ axis.

By looking at the figures, the first thing that is noticed is that top-down cracking is dominant in most of the cases as well as a high top-down crack propagation rate in general. This may be due to the very high values of concrete stiffness that have been input in the program for each state. They are all in the range of 22000 to $50000 \mathrm{MPa}$ which can be considered to be quite high. This is because they represent values of stiffness for a rigid pavement, as these composite pavements are a result of a rehabilitation. This can also be cross-referenced with Figure 22: Effect of variation of concrete elastic modulus to Nf, in Chapter 2 under subchapter (2.4.3), where it is shown that in higher range values of the concrete elastic modulus, dominant mechanism is top-down cracking.

The other thing that could be noticed is that development of reflective cracking at each location on the pavement sections is defined by a specific number of wheel loads and this differs from state to state. This is due to the different construction of each pavement section, for example it consists of different material properties. The previous state of surface of the pavement regarding the severity of pre-existing cracking before the placement of the asphalt overlay is another reason for the different reflective cracking development on each pavement section. Specifically, regarding the result from each state: 
Alabama: The first example shown is from Alabama. It can be seen that the dominant failure mode comes from the top-down cracking and that there is no cracking occurring in the other direction, at location 5 (red dashed line). However, the 1st location fails after around 2.2 million of number of wheel loads (1 year) and the 5th location after around 1.4 million (8 months).
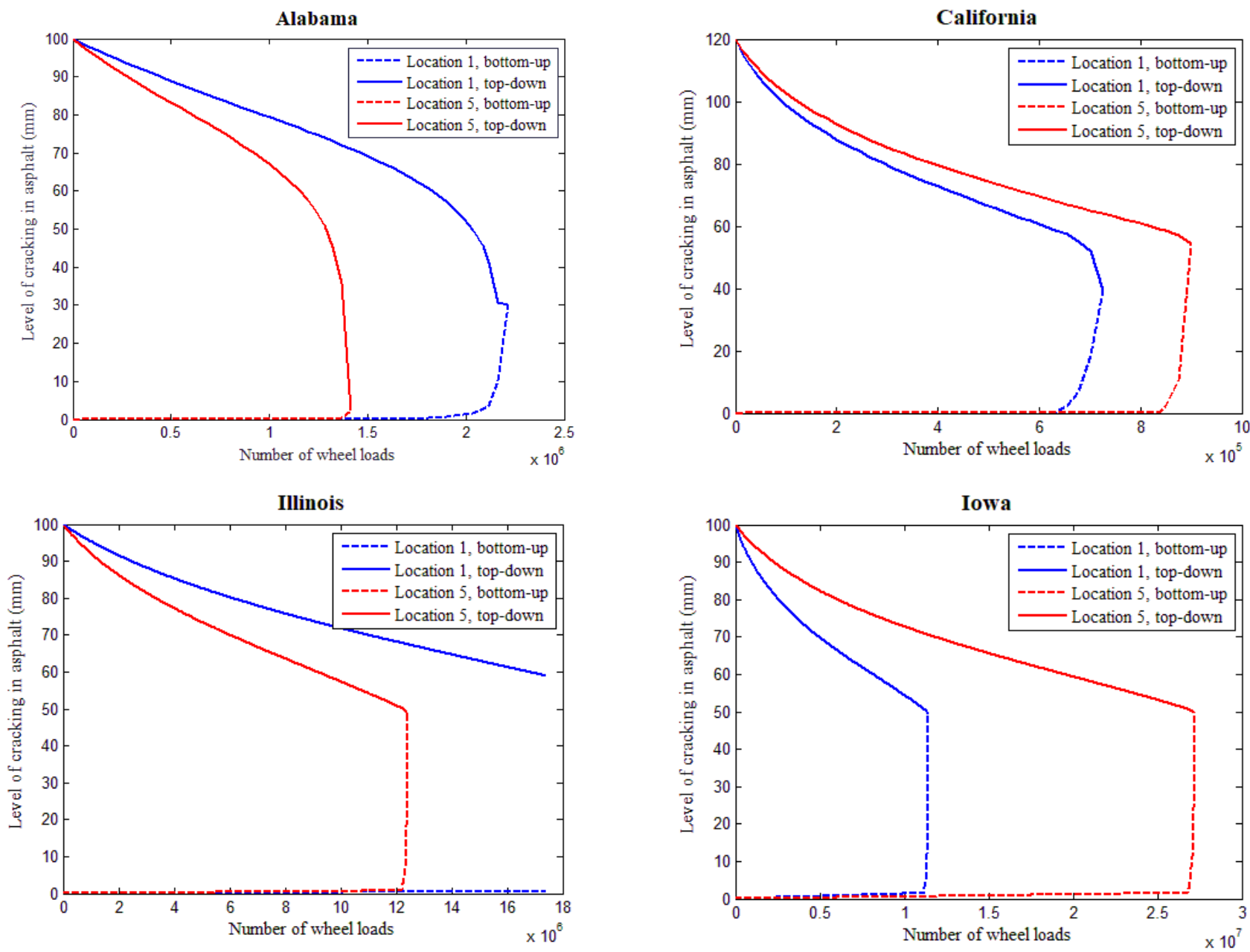

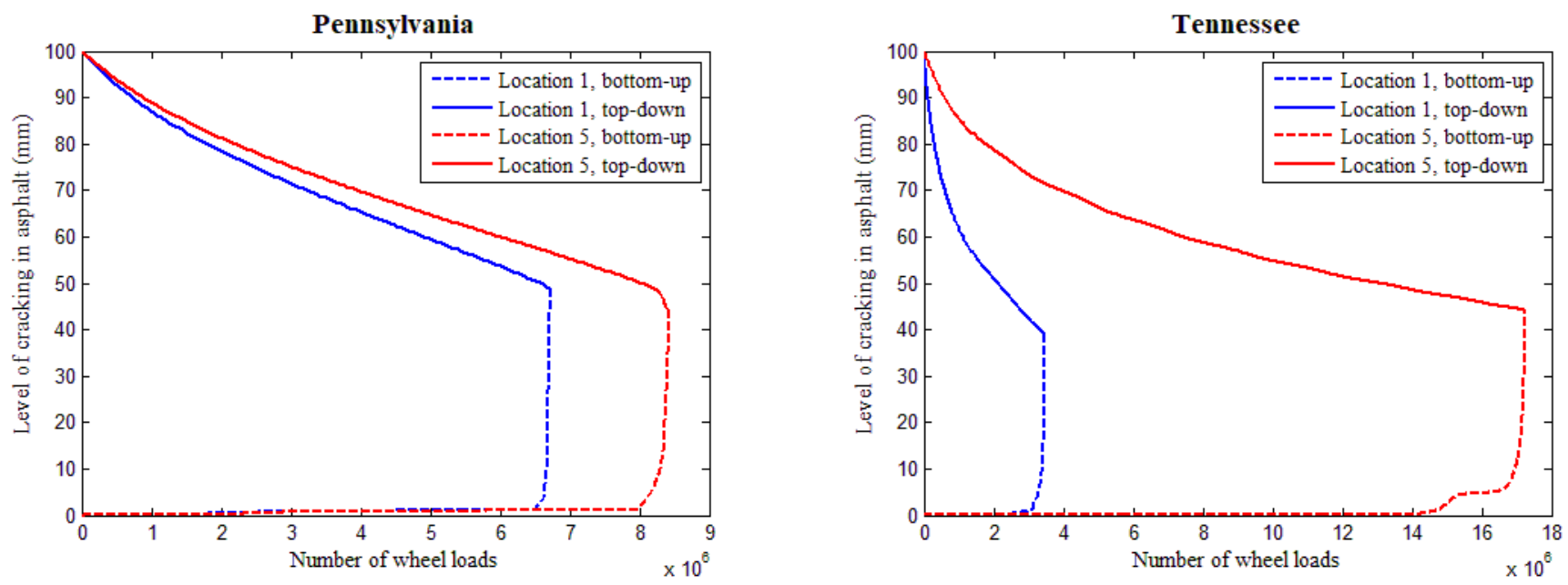

Figure 81(a-f): Bottom-up and top-down level of reflective cracking at $1^{\text {st }}$ and $5^{\text {th }}$ location.

(a: Alabama, b: California, c: Illinois, d: Iowa, e: Pennsylvania, f: Tennessee)

California: The locations in California show a similar situation. The dominant failure mode is topdown cracking. The difference is that less wheel loads are required for the locations to fail. These are around 720,000 number of wheel loads (8 months) for the 1st location and 900,000 number of wheel loads (11 months) for the 5th before each location fails. The reason for this could be the reduced thickness of the concrete layer and the subbase of the pavement section in California in comparison to the layer thicknesses of the pavement section in Alabama (The layer thicknesses can be seen in Table 27).

Illinois: The example locations that are given for the state of Illinois are different to what has been shown until now. The dominant failure mode may be determined as top-down cracking but cannot be said with certainty. The reason for that is that through 12 years of traffic the $1^{\text {st }}$ location shows that top-down cracking develops until the middle of the asphalt layer but no bottom-up cracking develops. From the $5^{\text {th }}$ location what can be seen is that top-down cracking develops until the middle of the asphalt layer. After reaching a certain point the tip of the top-down crack would be in tension therefore the stress distribution would change leading to the effective asphalt thickness being reduced. This leads to a higher bottom-up crack rate forcing the two cracks from opposite directions to meet.

Iowa: Regarding the locations of a pavement section in Iowa top-down cracking seems to be dominant leading to the same outcome as location 5 in Illinois. Bottom-up cracking is originally minimal and only develops in the end due to the development of top-down cracking and the appearance of tension. 
Pennsylvania: In this case the two locations shown develop cracks through the whole thickness of the asphalt with similar crack propagation rates. The time for each location to fail and develop these cracks just differs by 40 weeks. The dominant failure mode here seems to be top-down cracking as well.

Tennessee: The reflective cracking of the two locations shown for this state appear to be quite different. The first location develops top-down cracking fully quite quickly after 3.5 million wheel loads whereas the other after about 17 million wheel loads. Top-down cracking is the dominant failure mode in this state.

It can be concluded that regarding each pavement section its material properties and structural characteristics play an important role to the rate of development of reflective cracks. These examples were used to show that between the different pavement sections as well as between the locations of the same pavement section there are differences in the development of reflective cracking. They do not all crack at the same time and this can also be shown more clearly in the results later in this chapter.

In all the figures (Figure 81, a-f) it can be seen from these examples that the dominant mode of failure is top-down cracking. Unfortunately, it cannot be confirmed if that is true in reality because from the database we can only see distress maps of the surface before and years after the overlay. Which means only the cracks on the surface can be seen. In some of the cases both examples have cracked through in a relative normal amount of time like in Alabama, California and Pennsylvania. In other locations like Illinois, Iowa and Tennessee some of the locations did not crack through and therefore did not fail. This in some cases matches the real data from the LTPP database.

\subsubsection{Whole-life flexible composite model predictions}

Pavement sections from six states in the LTPP database have been selected to be simulated in order to apply the traffic and thermal model. These are Alabama, California, Illinois, Iowa, Pennsylvania and Tennessee and they were selected according to the data availability that could be used in the simulations and for the result validation. One typical pavement section belonging to each state is simulated each time. First the traffic-induced cracking model is applied and then the thermal-induced cracking model. The results are estimated and presented below. 
Weekly time increment was selected and after each week it was checked whether each location fails by checking if top-down cracking met bottom-up. The program would run until all potential locations fail for that pavement section (\%failure=100\%) or after the traffic loading of 12 years. The traffic data selected was arranged to last for 12 years because it is in adequate amount of time that case studies in the LTPP showed reflective cracking develops in most locations. In the following figures (Figure 82, Figure 83, Figure 84, Figure 85, Figure 86, Figure 87) the progression of the failing locations - locations that show reflective cracking - is shown. On the y axis is the percentage of failure and on the $\mathrm{x}$ axis is the time needed over a weekly time increment. The blue line shows the progression of the failing locations on each pavement section over a weekly time increment when dynamic loading is used. The light blue line shows the progression of failure when only static loading is used.

The data scattered as green circles represent the real data from the LTPP database. The number of transverse cracks on the surface of the pavement sections has been counted years after the placement of the asphalt overlay and the percentage of them over the total cracks that could have reflected is calculated along the years. These percentages are scattered over time in each graph, showing the progression of the real cracks along the years. In certain cases, the \% of transverse cracks counted on the surface are more than $100 \%$ because included in them are not only reflective cracks but also transverse cracks that develop between them in other locations.

By comparing the prediction (blue line) with the real data (green circles) it is noticed whether each prediction falls within a good range of the real data. In all the states studied it is shown that the progression is similar and quite close as it can be seen from the following figures. 


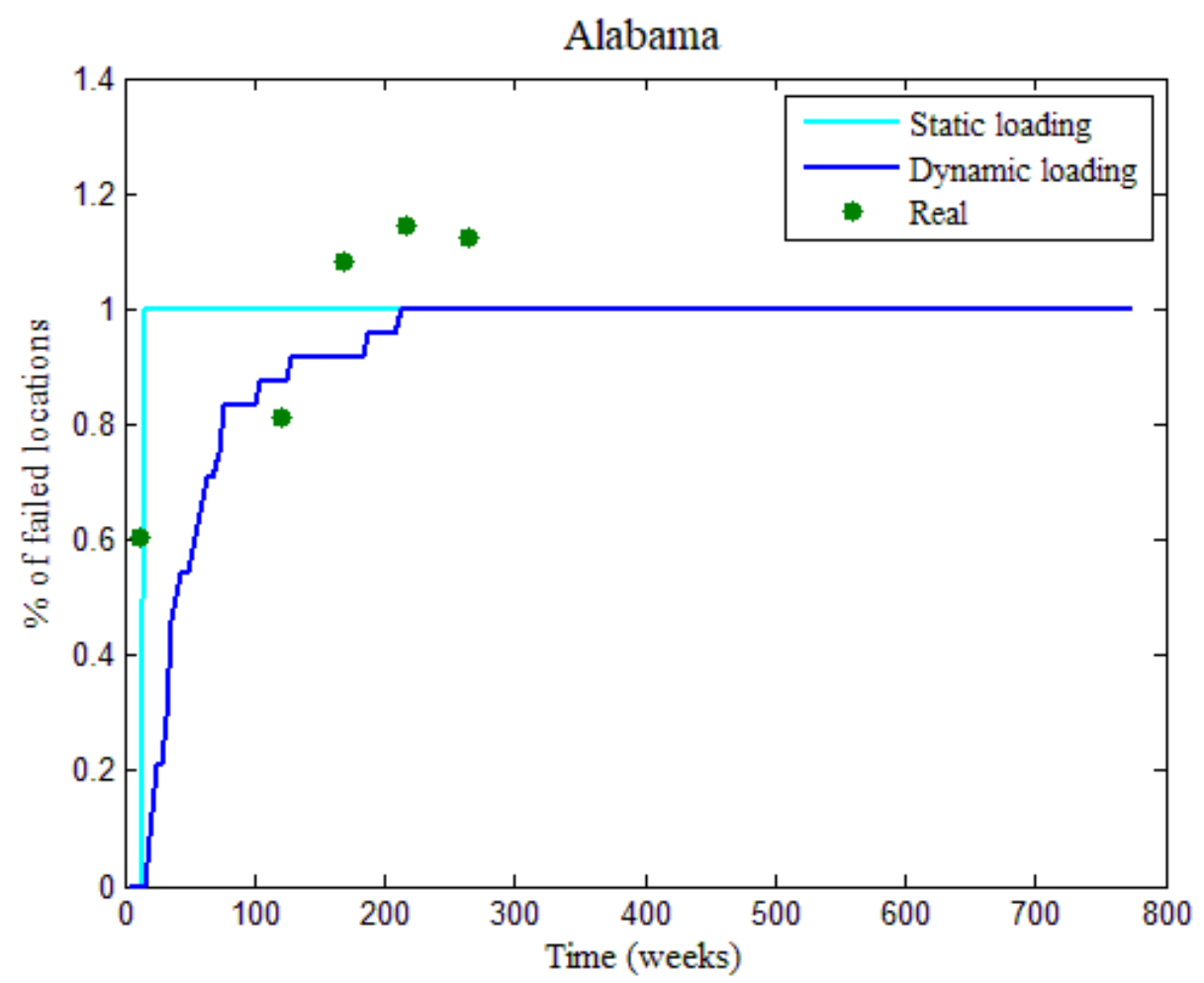

Figure 82: \% of failed locations over time due to dynamic and static traffic loading - Alabama.

We can see in the first figure (Figure 82) regarding the state of Alabama, that the real data percentage goes over $100 \%$. This is because in the data there are also included transverse cracks that develop in other locations excluding the potential ones which develop reflective cracking. The prediction shows that the locations start to crack around 40 weeks after the initiation of the simulation, whereas the real data shows high percentage of cracking already in the first few weeks. Around week 115 the prediction is very close to the real data in terms of percentage. After 150 weeks of wheel loading the real data percentage goes over $100 \%$ which means there is transverse cracking in more locations than the potential ones, meaning transverse cracking has developed on the asphalt surface in locations in between pre-existing cracks or joints. The prediction, on the other hand, continues slowly to show failure in more locations until finally reaching the 100\% failure after about 250 weeks. By comparing with the prediction due to static loading it is seen that all the locations fail at the same time and only a few weeks after the initiation. This shows that using static loading instead of dynamic can be unreliable compared to the real data to show progression of reflective cracking. 


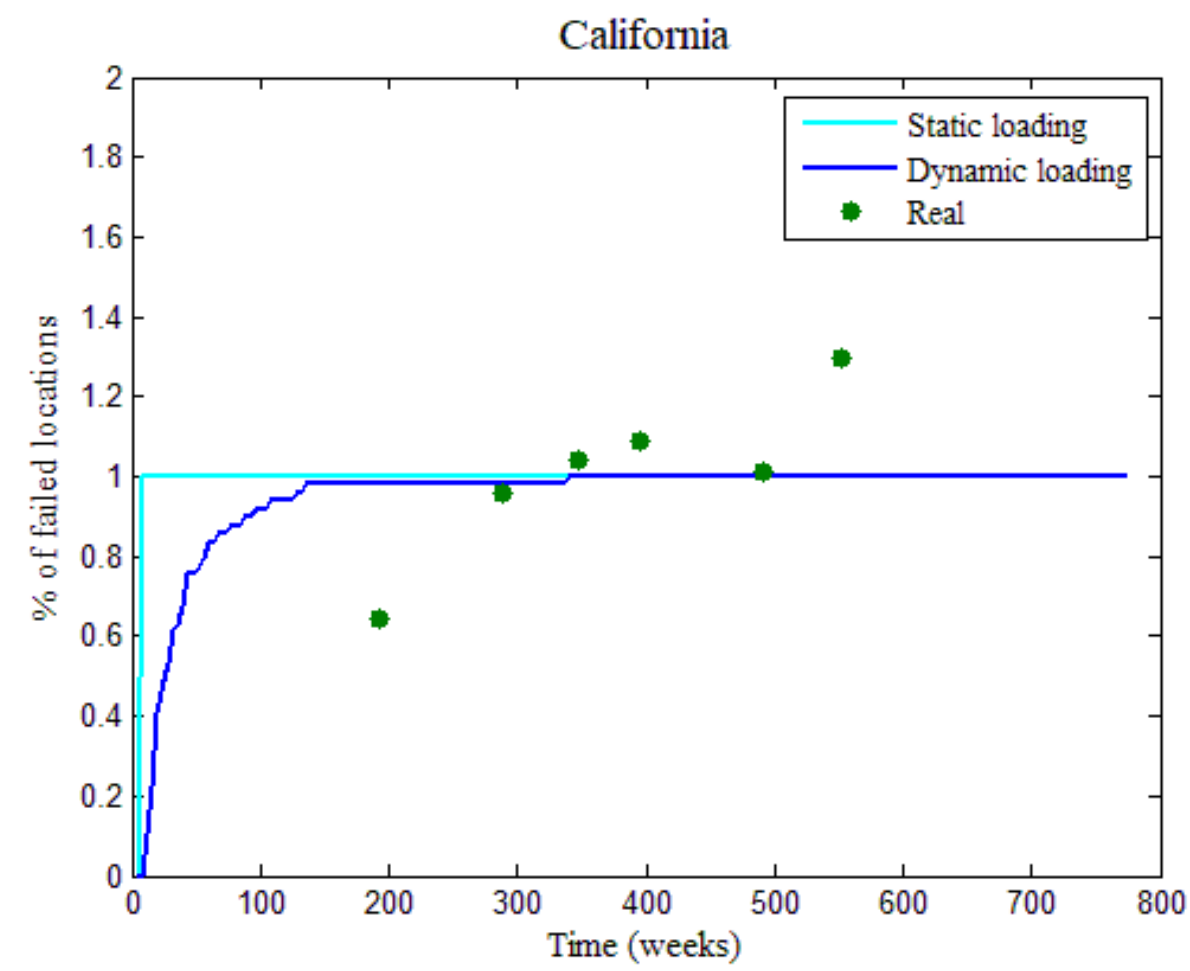

Figure 83: \% of failed locations over time due to dynamic and static traffic loading - California.

The data from the state of California (Figure 83) also shows transverse cracking in more locations than the potential ones and the \% failure reaches 100\% after around 350 weeks. The prediction percentage cracking due to dynamic loading shows steady development but it starts to match the real data at about week 300 , when both real and prediction reach $100 \%$. This could be considered as an indicator of the prediction matching the real data. Regarding the prediction due to static loading, all the locations crack at the same time only a few weeks after the initiation.

The real data regarding the pavement section in the state of Illinois shows on Figure 84 that for the most part all locations had not developed reflective cracking (up to week 500) and only in the end quite quickly $100 \%$ of locations having failed had been reached (week 600). The results from the prediction due to dynamic wheel loading reflect that because they do not show $100 \%$ of locations having developed reflective cracks. The prediction (blue line) shows that the locations start to show reflective cracking much later than it happens in reality (week 300). The best point in the simulation is around week 600 where real data shows $60 \%$ of locations having developed reflective cracking whereas the prediction shows 30\% (30\% difference). 


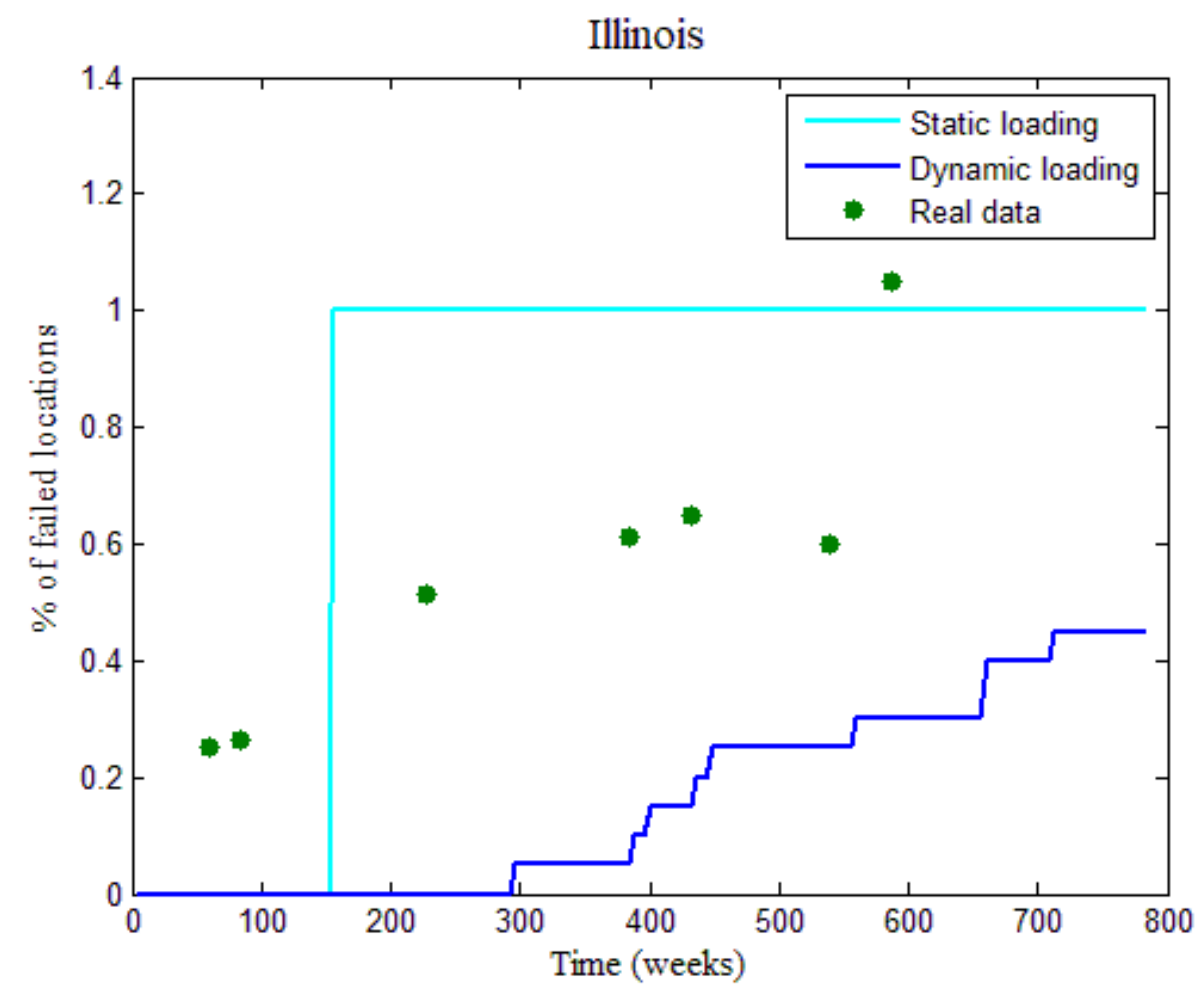

Figure 84: \% of failed locations over time due to dynamic and static traffic loading - Illinois.

Regarding the pavement section simulation of the state of Iowa, it seems that the real data cracking percentage is quite high from the beginning (40\% week 100) and originally it does not match the prediction due to dynamic loading (Figure 85). However, prediction and real measured data start to develop in a similar way especially after 300 weeks of traffic loading. Throughout the analysis as well as near the end, the prediction with the measured data differ at best only about $10-15 \%$. Unlike the previous cases, the prediction due to static loading does not reach $100 \%$ of locations showing reflective cracking immediately a few weeks after initiation, but instead $100 \%$ is reached after about 50 weeks of traffic wheel loading. It is also seen that the percentage of the real data reaches $95 \%$ around week 450 but is slightly reduced later on. That could be due to a number of transverse cracking no longer being measured as such but being covered by areas of fatigue cracking or block cracking. 


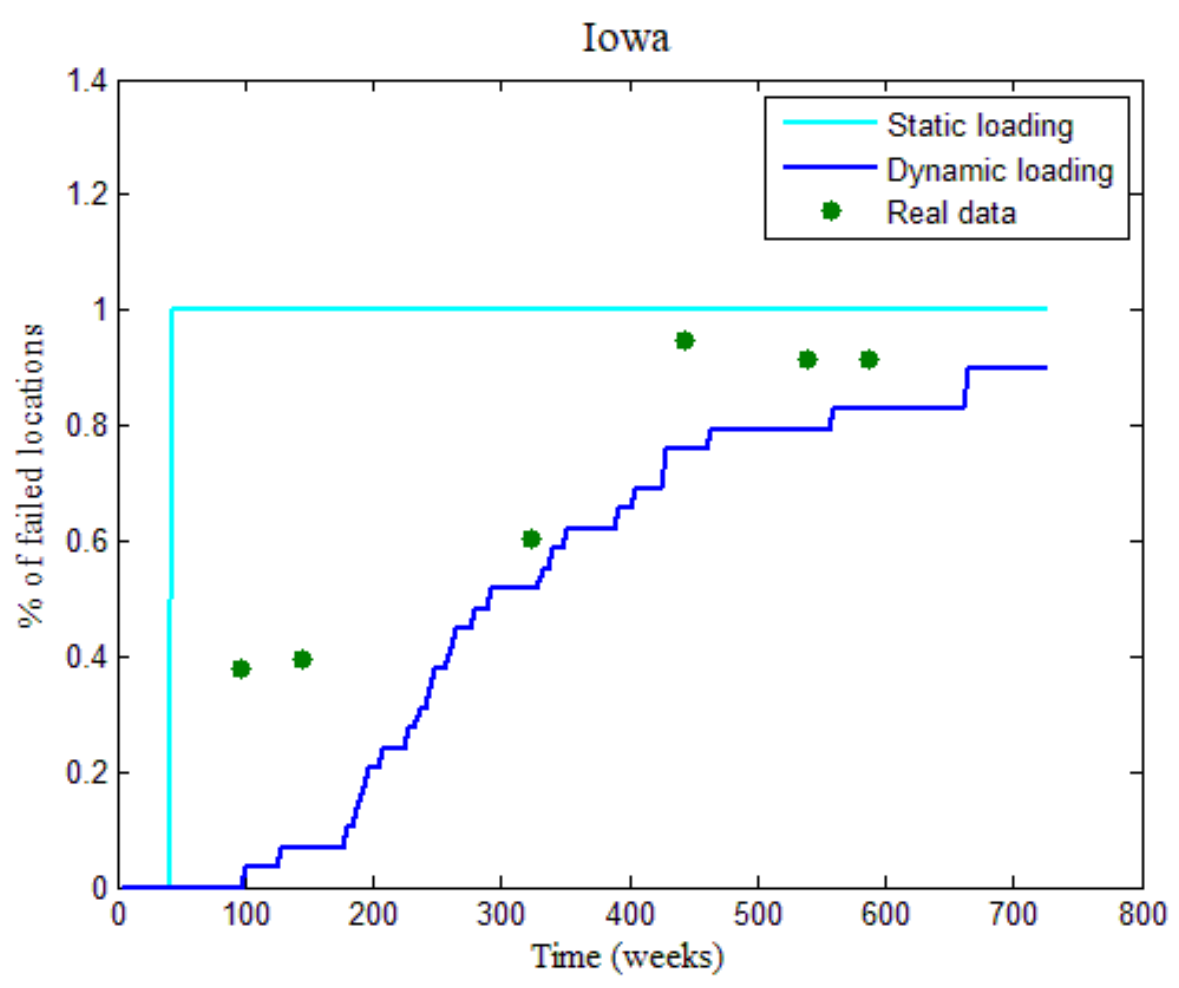

Figure 85: \% of failed locations over time due to dynamic and static traffic loading - Iowa.

For the state of Pennsylvania, after very few weeks, in Figure 86, the real data shows cracking in about $90 \%$ of the potential locations. The prediction due to dynamic loading shows a percentage in the range of $80 \%$ after about 200 weeks of wheel loading. In the beginning unfortunately, the prediction does not match the measured data, but in the end the results differ only $10 \%$ or less especially after week 200. 


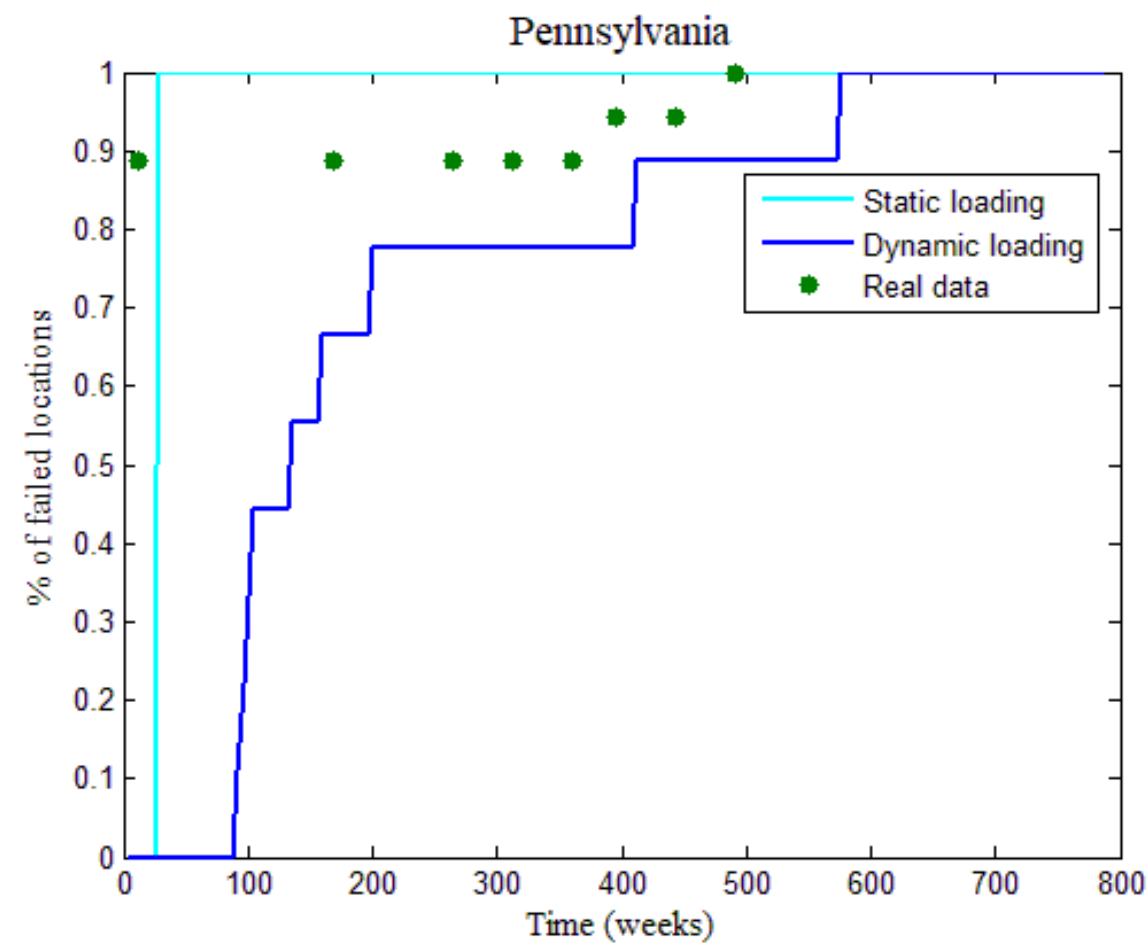

Figure 86: \% of failed locations over time due to dynamic and static traffic loading - Pennsylvania.

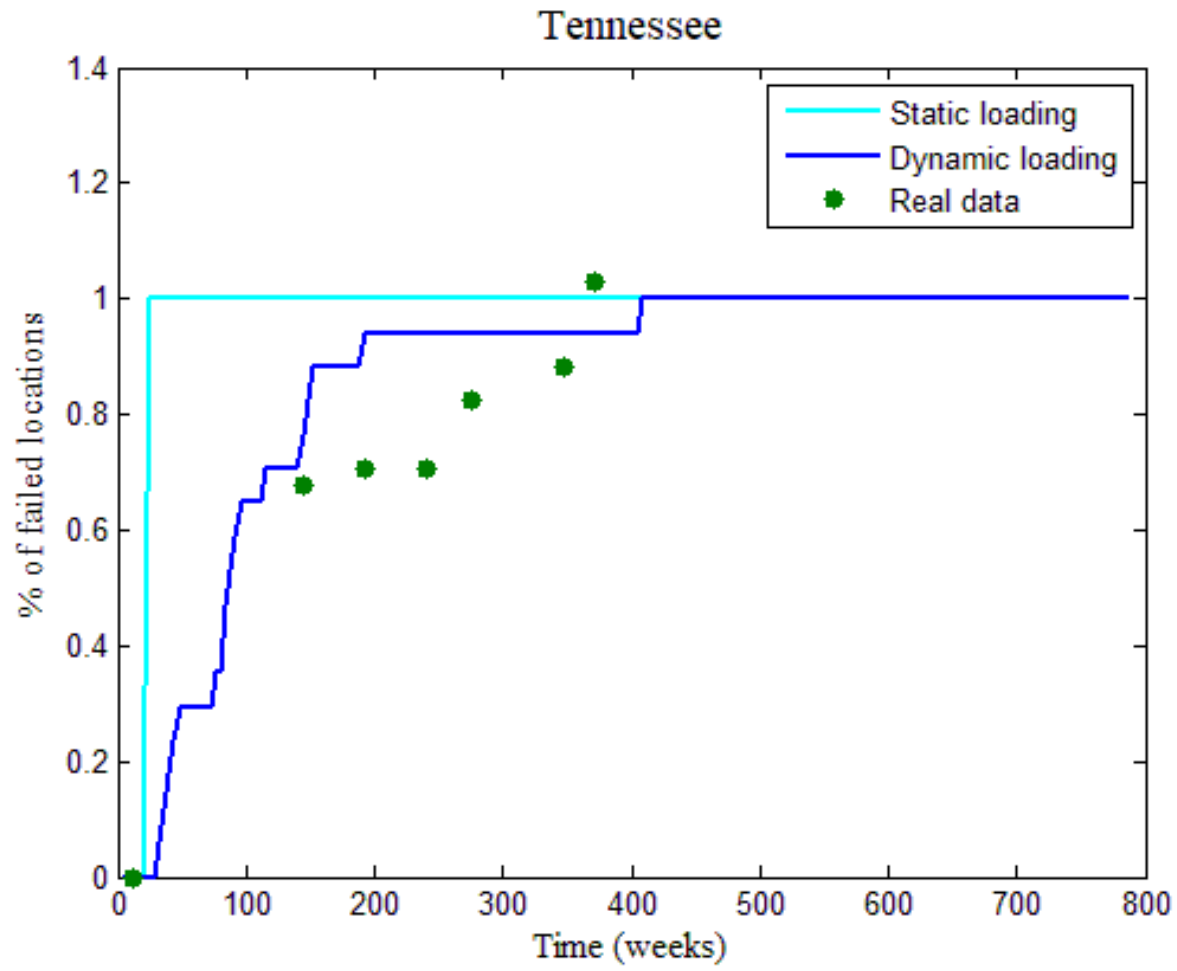

Figure 87: \% of failed locations over time due to dynamic and static traffic loading - Tennessee. 
The simulation of the pavement section in the state of Tennessee (Figure 87) showed that there are similarities in the way the percentage of locations showing reflective cracks progresses. The difference between prediction and measured data is around 10-25\% through most of the progression. The measured data shows more locations cracked on the surface than the potential ones, letting the percentage overcome the barrier of $100 \%$.

To summarize, the results in the above figures show the development of reflective cracking in the potential locations. The time when that happens differs for each location for both the prediction and the measured data. In general terms, it is seen that the prediction due to dynamic loading follows the real data in a range of $20-30 \%$, and even less at certain times, which can be considered as a relatively close range. It is shown that there is no point in trying to simulate the pavement sections and the traffic loading by using static loading. The results from that do not show something significant because all the locations crack at the same time.

\subsection{Thermal cracking model example simulations}

The thermal cracking model, works in daily temperature cycles and the result after the calibration is given in years to failure after 1 time increment. The consecutive outcomes from each day, given in years to failure, are converted to number of days to failure. With the help of damage mechanics, the damage due to thermal cracking after each day is estimated with the help of the following equation:

$$
\text { Damage }=\frac{\text { Number of cycles }}{\text { Number of days to failure }}
$$

When the total cumulative damage reaches the value 1 then it is considered that the pavement section has failed in regards to thermal cracking damage.

Below is presented the progression of the damage due to thermal cracking for example pavement section from 6 different states until it fails (Damage=1). What can be seen from this figure is the cumulative damage ( $\mathrm{y}$ axis) along time (in days $-\mathrm{x}$ axis). The point when this damage reaches 1 refers to the failure in regards to thermal cracking. 


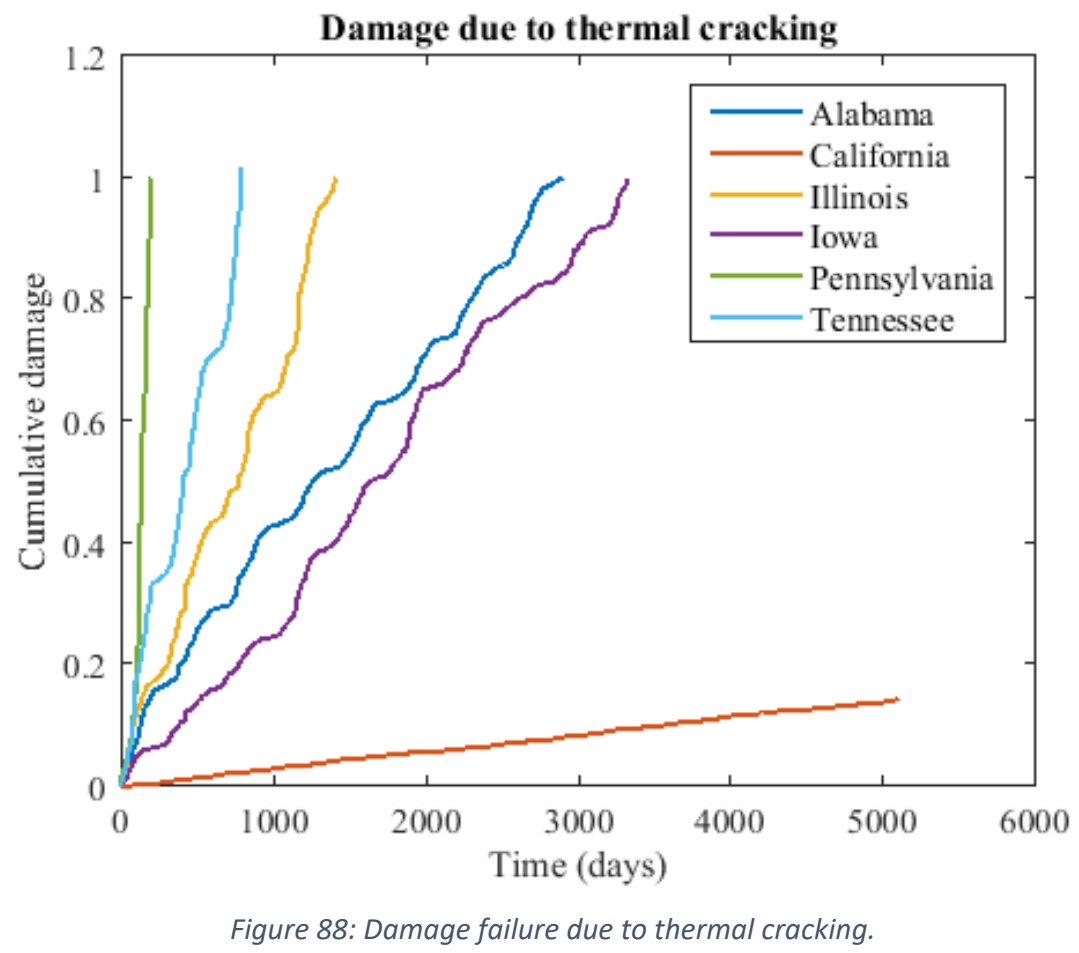

- What is pointed out is that the pavement section from the state of California is the only one that throughout the analysis the total damage of which does not reach the value 1 (over 5000 days). This is due to the short slab length of this pavement section which is $3 \mathrm{~m}$. in comparison to the slab lengths of the rest of the pavement sections (see Table 27). The length of the slab (also known as crack spacing) according to the parametric analysis of the thermal cracking model in Chapter 3 (3.5) is one of the most important parameters, the variation of which would have a great effect on the number of cycles to failure.

- Regarding the outcomes from the other states, it is noticed in Figure 88 that the one that fails first due to thermal damage belongs to the state of Pennsylvania (196 days). This matches the results from the traffic driven cracking model. The measured number of transverse cracks in Figure 86 revealed the appearance of transverse reflective cracks immediately after the placement of the asphalt overlay, determining a cracking behaviour which could be due to thermal damage.

- The real data from the state of Alabama also showed a high number of transverse cracks from the beginning of the placement of the asphalt overlay and all the locations showing reflective cracking after about 200 weeks (4 years) (Figure 82). However, the results in Figure 88 for 
this state show that around 3000 days (8 years) are required for the pavement to reach failure due to thermal damage.

- Regarding the rest of the states, failure due to thermal damage for the pavement section of Illinois occurred after about 1400 days, for Iowa after about 3300 days and for Tennessee after 750 days. The possibility for traffic driven reflective cracking to be dominant is higher for these states but it cannot be said with certainty.

\subsection{Summary}

In this chapter the simulations applied within the program are presented for pavement sections in 6 different states. The focus of the results is the prediction of a number of reflective cracks on the surface of the asphalt overlay at locations where cracks or joints in the underlying concrete layer of a rigid pavement pre-existed. The goal of this prediction is to compare the number of cracks that reflect on the pavement over the same time, for the real pavement sections and the simulations. The data for this comparison is found in the Long-Term Pavement Performance (LTPP) database. The measured data used was the number of transverse cracks on a pavement section, because reflective cracks are not given separately but included in the total number of transverse cracks.

The results are based on reflective cracking due to traffic loading and due to thermal loading. Regarding the traffic loading induced reflective cracking it is analysed both with the help of vehicle dynamic loading and static loading and the strong influence of the dynamic loading is shown in the results. A steady progression of reflective cracking can be shown over different locations of a pavement section due to the sinusoidal forces that originate from dynamic loading.

The way results are presented involve defining failure first of all at one location and also regarding reflective cracking of each pavement section as a total, when reflective cracks develop at all potential locations. At the same location when cracks developing from the bottom of the asphalt overlay and from the top meet, then it is considered that the crack has reflected, and therefore that location has failed. When this occurs at all the locations of a pavement section then it is considered to have failed as a section. The failure of these locations over time, until all of them fail, is shown and compared with the measured data. The prediction showed a strong resemblance with the measured data, showing 
the steady development of reflective cracking in a range of $10-30 \%$ of the real data regarding reflective cracking failure.

Failure due to reflective cracking from thermal loading is also covered. The thermal cracking model is applied for a pavement section in each of the 6 states and the goal was to show the time needed for a pavement section to fail due to thermal loading with the help of damage mechanics. The results showed comparable results for the state of Pennsylvania, which is actually the one that showed the appearance of reflective cracking immediately after the asphalt overlay placement, revealing a thermal cracking prone behaviour.

In summary, the results show a strong resemblance between the prediction of the failure progression due to traffic loading with the measured percentage of transverse cracking on the surface of the pavement section for some of the states. Particularly, there is a strong resemblance in the states of Alabama and Tennessee. Of course, this specific way of approaching and presenting the results stems from the need to accommodate the availability of data for validation in the LTPP database. 


\section{Conclusions \& Recommendations}

\subsection{Recap of objectives}

The need for developing composite pavements led to ongoing research in this field. One of the main concerns is the development of one of the main distresses on these pavements, reflective cracking. It would be significant to predict when and where these cracks develop because if they appear and remain untreated the increased roughness on the pavement or the possibility of water infiltration in the lower layers could deteriorate the condition of the pavement and make it unsafe.

The main aim of this research was the development of a validated model which would be used to predict distresses in flexible composite pavements over a long period of time due to both traffic and environmental loading. To do this a simplified mechanics-based model was required in combination with an existing vehicle dynamic model.

Another objective was the investigation into the parameters that affect the behaviour of a composite pavement, that could be among others structural parameters or loading parameters. The conclusions can be used in the development of design methods of a composite pavement.

\subsection{Conclusions \& Summaries by chapter}

\subsubsection{Chapter 2 - Modelling of reflective cracking}

Previous work showed that modelling of reflective cracking took place mainly with the use of finite element simulations and the use of a single static load. Fracture mechanics was also used frequently to model the crack propagation in the asphalt.

A simplified mechanistic model was selected to simulate composite pavements and model the mechanism of reflective cracking. OLCRACK is based on simplified mechanics equations and it has been implemented in MATLAB to investigate the progression of top-down and bottom-up cracking in composite pavement structures containing an asphalt surfacing.

The model that predicts reflective cracking due to traffic loading consists of two elements. The bottom-up cracking model and the top-down cracking model. At each location it is checked whether the two crack developments meet, meaning the crack would have reflected through the asphalt 
thickness. The top-down cracking model is more complex than the bottom-up due to the appearance of bending and shear stresses in the asphalt due to a wheel load.The program takes in account the crack propagation through the asphalt thickness and the asphalt elastic modulus is reduced after each crack development revealing a reduction in the asphalt stiffness.

Applying the simplified model on two case studies, a new composite pavement as well as a composite pavement from rehabilitation of a rigid pavement showed two different outcomes. Due to the high stiffness of the concrete layer in the rehabilitated pavement there was minimal development of bottom-up cracking, compared to the new composite pavement where cracks developed from both directions.

A parametric analysis has been undertaken to determine the most influential variables. Those were found to be the asphalt elastic modulus and the asphalt layer thickness. They were both found to increase the pavement's life as they were increased, which is easy to understand. However, the results were also sensitive to the concrete elastic modulus and the concrete layer thickness. It can be concluded that the material and structural properties of each pavement play a significant role in the duration of the pavement's life.

It was also considered important to examine the dominant mode of cracking, bottom-up or top-down in each case. It was concluded that when bottom-up cracking was dominant the pavement's life would increase while increasing the value of a specific factor. Also, at the point of change of mechanisms it was found that it would affect the pavement's life varying the rate with which the number of wheel loads to failure changed.

\section{Conclusions}

- It was found that it was best practice to work in a programming environment like MATLAB so the model to be modular and adaptable.

- Simplified mechanics based models were selected for the sake of computing time and due to the complexities of the development of reflective cracking.

- The simplified crack development mechanisms on which the whole model is based is helping with the fact that it can be combined with dynamic vehicle modelling. 
- Both bottom-up cracking and top-down cracking develop at the same time at the same location until they meet and it is considered that the crack has reflected. That proved to be useful as it is the way to define the 'failure' of a specific location.

- Material layer properties are considered very important as a less stiff asphalt layer for example could show quicker progression of reflective cracking development compared to a stiffer one. The appearance of reflective cracking could be delayed depending on the material layer properties.

- The fact that bottom-up cracking or top-down cracking could be dominant is important and affects the rate with which the pavement will show reflective cracks

\subsubsection{Chapter 3 - Modelling of thermal reflective cracking}

Review of previous work showed that there has been little investigation in thermal reflective cracking. The selected model, ThermCrack, assumes that daily thermal cycles would result in the damage of a composite pavement. Repeated sinusoidal temperature cycles are applied and the strains in the asphalt are calculated and used to estimate the number of temperature cycles to failure.

The approach is based on the one-dimensional heat flow equation, taking into account of viscosities of the materials with the temperature changes in hourly time steps. A fatigue equation is used to calculate the number of wheel loads to failure.

The development of thermal strain in the pavement layers depends significantly on the thermal expansion coefficient of each layer as well as the length of the underlying slab of each pavement section.

\section{Conclusions}

- Daily temperatures of the pavement are required for the thermal cracking model in order for the temperature distribution to be estimated through the pavement layers.

- The crucial temperatures required for the analysis are at the top and bottom of the asphalt layer.

- The thermal expansion coefficient of each layer are critical as they can affect the amount of expansion each layer goes through and as a result the strains that develop due to that. 
- There are certain limitations in regards to the thermal model. These have to do with the data availability. Also, they regard the daily temperature variation and the fact that damage is only considered by a damage law.

\subsubsection{Chapter 4 Dynamic vehicle model}

In this study an attempt is made to use vehicle dynamic loading instead of static loading to study the development of reflective cracking on composite pavements going away from traditional methods like the ESAL approach.

The vehicle dynamic model includes a random surface profile generation and the use of 9 different quarter-car models. These are based on different sprung and unsprung masses as well as different truck speeds based on relevant data from LTPP.

The vehicle dynamic loading takes in account the roughness of the pavement which is affected by the appearance of transverse cracks on the surface of the asphalt layer and is updated accordingly. In order to do that, correlation equations were extracted from data regarding the number of transverse cracks on a pavement section in relation to the International Roughness Index (IRI). The IRI was shown to increase after the appearance and development of these cracks and therefore could be included as a feedback mechanism in the vehicle dynamic model. This way, the dynamic vehicle model is used with the help of actual IRI data from the real sections in the LTPP database.

\section{Conclusions}

- 9 different vehicle models have been used which are characterised by different speed and mass. These cover a range of vehicles that pass on the sections from the LTPP database.

- The model can be easily modified concerning the type of vehicles used (weight, category) as well as speed of the vehicle.

- Roughness of the pavement surface is considered very important in the development of the specific forces that are applied on the pavement. That is why it should be taken in account in the analysis of a deterioration of a pavement. This can be done with the help of vehicle dynamic loading. 


\subsubsection{Chapter 5 - Whole life long-term flexible composite pavement model}

In this chapter the various sub models that have been described in previous chapters are combined in one whole model. The framework of the model is presented including: the input, the mechanisms of reflective cracking, the dynamic vehicle model.

An important aspect of this chapter was the identification of a way to define failure of a composite pavement section under examination. It was concluded that the best method for this was to identify the development of reflective cracking in all locations where underlying transverse cracking existed in the old concrete layers. If reflective cracking develops at all locations then $100 \%$ of failure is reached for that specific pavement section.

This was also taken into account in the model flowchart presented in this chapter. The failure progression is shown over time, and the analysis stops when $100 \%$ is reached. This method was considered best so the effect of the dynamic vehicle model can be considered. In this section also, the basic time loops with which the program works are presented. The first represents the frequency with which a new crack length develops. This results in new updated values of crack length and of the asphalt elastic modulus. The other loop represents a wider time frequency over which the International Roughness Index is updated.

It is concluded that the whole model is modular and therefore it is relatively easy to modify it depending on the needs of research. For now, it is developed this way to accommodate the availability of data from the Long-Term Pavement Performance database which contributes to the needs of its validation.

An example of an application of the model is also given, to show step by step the procedure, with the input required followed by the outcomes. The analysis runs until all potential locations develop reflective cracking in order to present $100 \%$ failure of the pavement section.

\section{Conclusions}

- The best way to identify failure in composite pavements was to check whether all the underlying locations with cracks would reflect on the surface. That would mean that the pavement section has 'failed' completely. 
- After looking at the whole life performance model it can be concluded that failure can be shown over time as vehicles pass.

- Extra attention should be given to the different time loops on which the analysis is based on because they are the ones that make this mode dynamic due to the fact that they are updated after each increment. These are the time frequency with which a new crack is estimated and that the asphalt modulus is reduced due to the appearance and growth of a crack. The other time frequency is the one that depends on the IRI update.

- In conclusion, the whole model has successfully combined both reflective cracking mechanisms with a vehicle dynamic model.

\subsubsection{Chapter 6 - Parametric Study}

The first part of this chapter entails a convergence study to investigate the ideal time increment for the two time loops included in the whole model. It was decided to use the weekly time increment for a new crack development as the outcome is still granular without compromising on the computation time. Regarding the other time loop, which represents the frequency with which the IRI is updated and new dynamic tyre forces are calculated, a monthly time frequency was selected.

The parametric study of the whole model showed that the most important parameters influencing the results are the asphalt elastic modulus and the asphalt layer thickness. It was shown that for most parameters a greater amount of time is required for the final 25\% (from 75\% to 100\%) to be reached. From this it may also be concluded that full failure at all locations may not be reached.

\section{Conclusions}

- It was understood that different time frequencies included in the model would have as a result different outcomes from the model. Therefore, after conducting a time convergence study the ideal time frequencies with which cracks develop and roughness is updated were estimated.

- From the different parameters studied it was found that the most crucial ones were the ones concerning the asphalt layer properties, the elastic modulus and the thickness. This could help in the case of rehabilitation of rigid pavements to decide on the actual properties of the new asphalt layer overlay in order to get the required outcome (pavement life). 
- Not all locations with underlying cracks will show reflective cracking, either due to it developing from bottom-up only or due to the different parameter properties from location to location.

\subsubsection{Chapter 7 - Validation \& Results}

An effort was made with the help of measured data to predict the progression of reflective cracking. Six states were used as case studies according to the availability of their data in the LTPP database. These states were Alabama, California, Illinois, Iowa, Pennsylvania and Tennessee. The required data extracted from the LTPP database were based on structural data, distress monitoring data, climatic and traffic data.

The distress of transverse cracking was the main distress of interest because in the data of transverse cracks were also included the transverse reflective cracks. Data showed the increase of transverse cracking throughout the years, as well as reflective cracking. Some distress maps from the LTPP database were also presented to confirm the appearance and development of reflective cracks.

In order to understand the results, the main concern was to adjust them in order to be easily compared to the measured data given in the LTPP database. The main issue was the availability of information regarding the number of transverse cracks on the surface of the pavement and no information regarding the dominant mode of cracking being top-down or bottom-up. For this to be taken in account, one pavement section of $150 \mathrm{~m}$ had a specific number of locations where reflective cracks could develop. The progression of the reflective cracking over time in reality was compared to the progression of locations showing reflective cracks from the predictions.

The appearance of a reflective crack on the asphalt layer of the composite pavement was defined as a failure of that specific location and it occurred when the bottom-up crack met the top-down crack and no further development could happen. Depending on the properties and structural characteristics of the pavements of each state, cracks had reflected on the surface at different times and after a different amount of traffic having used the pavement.

One important characteristic of this model is that after the first time increment a crack is always created from bottom or from the top of the asphalt layer. And after each consecutive time increment 
these cracks progress and develop by a specific crack propagation rate. There is no choice in the program that no crack would develop, or that the crack would progress only from the top or only from the bottom and not in the opposite direction. That is an approximation included in the program.

From the simulations it can be concluded that the predominant mode of failure was reflective cracking from the surface of the asphalt layer (top-down cracking) and that cracking from the bottom of the asphalt layer would develop usually at very small rate initially. This cannot be validated though, because, due to the data availability there is no actual way to acknowledge whether the reflective cracks originated from the top or from the bottom.

Also, it can be concluded that the static loading when applied in comparison to the dynamic loading shows very little information regarding the progress of the reflection cracking on all the pavement section. It can only be used at one location but then again it shows a very quick development of reflection cracking at that location and cannot be considered reliable. It would not be considered as a useful tool to analyse the reflective cracking progression of a whole pavement section but just individual locations.

\section{Conclusions}

- The range of pavement sections selected are all rehabilitated rigid pavements with an asphalt overlay placed at the end of their lives with pre-existing transverse cracks on the concrete layer.

- Potentially, it can also be applied for newly constructed flexible composite pavements, which will require different material properties to be input. This might show quicker progression of the development of reflective cracking over a range of time due to the weaker base layer compared to the rigid pavement.

- From data analysis it was shown that transverse reflective cracking definitely developed over time on these pavement sections with different progression for each case study. That is the result of different materials, structure and environmental conditions of each case study.

- The predominant mode of failure shown in the results was top-down reflective cracking in most cases with bottom-up cracking having a slower progression in certain cases. This is due to the concrete rigid base, which makes movement and internal stress transfer more difficult. 
Top-down cracking on the other hand also has an extra shear stress on the surface due to the wheel load. That increases total strain and results in quicker deterioration.

- The model has been applied for two climatic regions: wet/freeze zone, wet/non-freeze. From the results it was found that the states of Alabama, California and Tennessee, which belong to the wet/non-freeze climatic zones showed that predictions were in a close range compared to real measured data. Regarding the two states representing wet-freeze zones (Illinois and Iowa) the prediction outcome did not match totally the real measured data.

- The range of crack spacing for which best results were shown is 3-8m. for the climatic zone of wet non-freeze. This is an indication of what crack spacing would be best to use, so the outcome prediction is closer to real reflective cracking progression.

- It was shown that the model is sensitive to certain input parameters. It can be firmly said that this model can be used for pavement sections for which there is relevant available data because of their crucial importance to the final results as was seen from the parametric study.

- Specifically, the state of the underlying pavement layer should be known so the crack spacing could be identified, and therefore the locations where potential reflective cracks can develop. This model could be used on the SPS sections (Specific Pavement Sections) for which most data is available.

\subsubsection{Results summary}

From the final results regarding the prediction of the \% of cracks having reflected out of the total number of locations that could develop reflective cracking, the results seemed close to the real measured data, showing a similar progression along time. The difference was of a range of 20-30\% and even $10 \%$ or less in best cases, meaning that if at a particular time the prediction and the measured data showed only a $10 \%$ or even a $20 \%$ difference in their value, then that can be considered as a good approximation of the real case. Specifically:

- The pavement section in Alabama, showed a very close resemblance of the results to the real pavement especially in the beginning of the analysis where their difference is only around $10 \%$. It seems that in the real case when $100 \%$ of reflective cracking is reached then the prediction shows a percentage of $85 \%$, leading to a difference of $15 \%$ at that point. 
- In the case of California even though the difference between real case and prediction is around $30 \%$ in the beginning, later on in the analysis both cases reach $100 \%$ around the same time.

- The worst case was that of Illinois that the prediction showed little development up to $30 \%$ of reflective cracking on the pavement section whereas the measured data showed up to around $60 \%$ of reflective cracking. However, that is not considered as a bad prediction because both prediction and real case showed for most part of the analysis that not all underlying cracks reflected on the surface of the pavement section, never reaching $100 \%$. It is a possibility that there are bottom-up cracks in the asphalt layer in reality that actually could not be discovered and were not measured.

- The pavement sections in the states of Iowa, Pennsylvania and Tennessee are the best cases showing a close resemblance between the prediction results and the real data. Specifically, in the state of Iowa for the second half of the analysis the difference is around 15\% or less.

- Regarding the state of Pennsylvania, in most of the analysis the results are really close, around a $10 \%$ difference with the real case.

- And finally, regarding the pavement section of Tennessee, the difference between prediction and results remain steady throughout most of the analysis, with that difference being around $20 \%$ or less.

- Regarding the thermal cracking damage, it was seen that the prediction for the pavement section of Pennsylvania, would match with the real measured data of number of transverse cracks having reflected.

\subsection{General conclusions}

Using this model provided a better understanding of the mechanisms of reflective cracking. In regards to this type of distress it is easier to expect where reflective cracking will develop. By knowing how the surface of the underlying layer looks like (pre-existing cracks and joints on the concrete base) we know where the potential reflective cracking can develop. Depending on the case, not all potential locations show reflective cracks and sometimes more cracks develop in areas in between and take the form of simple transverse cracks. 
These potential locations are also what allowed this model to be compared with the real measured data. Unfortunately, in the database are included only number of transverse cracks on the asphalt overlay. In order to measure the number of reflective cracks the procedure would be to compare the distress map from before the placement of the asphalt overlay with the distress map after the placement. However, that would be quite a complex procedure due to the fact that not only reflective cracks are depicted on the distress map after the placement of the asphalt overlay but also other types of cracks like transverse cracks or fatigue cracks.

This whole-model by combining mechanical responses of composite pavements and vehicle dynamic loading can potentially be used for the prediction of reflective cracking in the locations of pre-existing cracks or joints in the underlying layers. With further improvements, and combination of the reflective traffic driven cracking model with the thermal model, as well as the use of appropriate input data, there is confidence that it could predict approximately the amount of time needed for reflective cracking to appear at all potential locations.

\subsection{Recommendations}

\section{Limitations}

The whole life model has so far been used and has given realistic results for composite pavements with a subbase which consists of unbound material with an elastic modulus in the range of 200400MPa. However, there are composite pavements in the same category of rehabilitated rigid pavements which contain a subbase that consist of cement bound material. This indicates a material with high subbase elastic modulus. When the model was applied for pavements with this type of subbase, it did not give realistic results, therefore this limits the use of this model to composite pavements with subbases with unbound material.

The other limitation which was mentioned earlier is that the prediction of the total reflective cracks on the surface of the pavement has been compared with the total number of transverse cracks on the pavement sections in the LTPP database and not the actual number of reflection cracks. In the LTPP database there are not included data regarding transverse reflective cracks separately, but only as a total of transverse cracks. It has been noted that when the total \% of reflective cracks from the data input is over $100 \%$ it means that in this number are included extra transverse cracks that occurred in 
between reflective crack locations. The way to overcome this is only by studying and analysing the distress maps of the surface of the pavement sections along time, comparing the distress map from before the asphalt overlay with the distress map from after the overlay.

In addition to the previous point, for the model to give realistic results the crack spacing of the underlying base is essential so the points where reflective cracking will potentially develop can be identified. The model is set to predict reflective cracks at the specific locations where the pre-existing underlying cracks are situated and it cannot take in account that cracks can reflect possibly up to $1 \mathrm{~m}$. from the original location where the crack existed.

The pavement sections which have been used as case studies have been selected also because no extra rehabilitation techniques have been applied to them during the placement of the asphalt overlay. For example certain pavement sections have gone through the techniques of crack and seat, saw and seal or concrete rubblization. These pavements have been excluded from the previous analysis because reflection cracking mitigation techniques have been used and would affect the outcome.

\section{Recommendations \& future improvements}

The model in order to be enhanced and to acquire more realistic results would require certain future work. Depending on the data availability the simulation of pavement sections in more states would be good so the conclusions would cover a greater range of composite pavement structures like for example ones that belong to different climate zones. This would depend on the data availability from the LTPP database regarding the pavement sections of each state. Certain assumptions would possibly be required for that.

It is recommended that the damage from the thermal cracking model be combined with the outcome from the traffic driven reflective cracking model. The failure in terms of reflective cracking from the traffic driven model is defined when bottom-up cracks meet top-down at the same location with no use of damage mechanics law. The way to combine the two loading mechanisms (thermal and traffic) would be using damage mechanics law for the traffic driven model as well, so when the total damage from both thermal and traffic model reaches 1 , it would mean that the pavement section has reached failure for this combination of loading. Now, the damage from thermal loading and the damage from 
traffic loading are presented separately and it is not clearly defined which mechanism is dominant in certain cases.

It should be mentioned that two issues have not been considered until now in the damage mechanisms. These are the mechanisms of ageing and hardening of asphalt. The asphalt material as it has been mentioned is a viscous material, sensitive to the daily and seasonal temperatures. Therefore, that would affect the opening and closing of the cracks of the asphalt overlay and due to that affect the time needed for reflective cracks to appear. Ageing can be included in the form of a factor which will affect the results by increasing or decreasing the pavement's life. Further investigation is required in this matter, through certain factors that can be taken in account to increase the asphalt stiffness at a specific year frequency. This will result in a reduction of the pavements life when hardening takes place.

The result of the developed program is to simulate composite pavements in order to predict the appearance of reflective cracking which would lead to the pavement's 'failure'. A distress that appears on asphalt layers which has not been considered yet in this whole-life model is rutting. In further studies it can also be considered by taking in account the average rut depth at points along the pavement due to wheel loading. This could be achieved in the form of a sub-model that would be included in the whole-life model and would contribute to the total failure.

This model is based on estimating the failure of a whole composite pavement section in terms of reflective cracking by taking in account the different locations where reflection cracking can potentially develop. These locations however, even though might be characterised by different properties in reality (material, layer thickness etc.) are not differentiated in some way except from the random generation of forces at each location. An average value is used for the definition of these parameters on a pavement section. So, a further improvement would include variation of these parameters along the pavement section for more realistic representation. To do that the pavement section could be separated in sub-sections of an equal length to investigate the state of the structure at each of these points.

Lastly, spatial repeatability is a factor that has not been included in the whole life model but has been seen that it can affect the results. It takes in account the state of the pavement along with the specific 
vehicle characteristics. It has been found that a vehicle of a specific speed could generate a spatial distribution of forces that could be repeated in following runs of similar vehicles. The pavement then would show specific peak spots where the damage would be worse than if spatial repeatability is not taken in account. 


\section{References}

AKBARIAN, M. (2012). Model Based Pavement-Vehicle Interaction Simulation for Life Cycle Assessment of Pavements.

Applied Research Associates (ARA), Inc. (2012). Validation Report - Tennessee SPS-6. Ref. 00720. [online] City: Publisher, Page 6 Available at: https://infopave.fhwa.dot.gov/ [December, 2016].

BRADEMEYER, B.D. (1988) ANALYSES OF MOVING DYNAMIC LOADS ON HIGHWAY PAVEMENTS: PAR II-PAVEMENT RESPONSE. In: International Symposium on Heavy Vehicle Weights and Dimensions Transportation Association of Canada.

BROWN, S., THOM, N. and SANDERS, P. (2001) A study of grid reinforced asphalt to combat reflection cracking (with discussion). Journal of the Association of Asphalt Paving Technologists, 70.

BROWN, S. (1973) Determination of Young's modulus for bituminous materials in pavement design. Highway Research Record, (431).

BROWN, S., BRUNTON, J. and STOCK, A. (1985) The analytical design of bituminous pavements. In: Institution of Civil Engineers, Proceedings, Pt2.

BRUNTON, J.M. (1983). Developments in the Analytical Design of Asphalt Pavements using Computers.

CEBON, D. (1993). Interaction between Heavy Vehicles and Roads.

CEBON, D. (1999) Handbook of vehicle-road interaction.

COLE, D. and CEBON, D. (1992) Spatial repeatability of dynamic tyre forces generated by heavy vehicles. Proceedings of the Institution of Mechanical Engineers, Part D: Journal of Automobile Engineering, 206 (1), pp. 17-27.

COLLOP, A.C. and CEBON, D. (1995) A Model of Whole-Life Flexible Pavement Performance. Proceedings of the Institution of Mechanical Engineers, Part C: Journal of Mechanical Engineering Science, 209 (6), pp. 389-407. 
COLLOP, A. and CEBON, D. (1995) A theoretical analysis of fatigue cracking in flexible pavements. Proceedings of the Institution of Mechanical Engineers, Part C: Journal of Mechanical Engineering Science, 209 (5), pp. 345-361.

COLLOP, A. and CEBON, D. (1995) Modelling whole-life pavement performance. Road Transport Technology. University of Michigan, Transportation Research Institute, 4, pp. 201-212.

COOK, J. and ELLIS, S. (2005) Design \& Maintenance Guide 33: Reflection cracking on airfield pavements - A design guide for assessment, treatment selection and future minimization. Ministry of Defence, TRL

(https://assets.publishing.service.gov.uk/government/uploads/system/uploads/attachment_data/file/5 52411/DMG_33.pdf)

DAVE, E. et al. (2007) Reflective and thermal cracking modelling of asphalt concrete overlays. Advanced Testing and Characterization of Bituminous Materials, 2, pp. 1241-1252.

DAVE, E.V. and BUTTLAR, W.G. (2010) Thermal reflective cracking of asphalt concrete overlays. International Journal of Pavement Engineering, 11 (6), pp. 477-488.

DAVIS, L.E. and BUNKER, J.M. (2008) Load-sharing in heavy vehicle suspensions: new metrics for old.

Design Manual for Roads and Bridges (DMRB), Volume 7: Pavement Design and Maintenance, Session 1: Preamble, http://www.standardsforhighways.co.uk/dmrb/vol7/section1/hd2399.pdf (last accessed 30/07/14).

DOH, Y.S., BAEK, S.H. and KIM, K.W. (2009) Estimation of relative performance of reinforced overlaid asphalt concretes against reflection cracking due to bending more fracture. Construction and Building Materials, 23 (5), pp. 1803-1807.

EISENMANN, J., BIRMAN, D. and HILMER, A. (1987) Effects of commercial vehicle design on road stress-research results relating to the roads. Strasse Und Autobahn, (Translated by TRRL as WP/V\&ED/87/29), 37 (6), pp. 238-244. 
ELSEIFI, M.A. and AL-QADI, I.L. (2004) A simplified overlay design model against reflective cracking utilizing service life prediction. Road Materials and Pavement Design, 5 (2), pp. 169-191. GARZON, J., DUARTE, C.A. and BUTTLAR, W. (2010) Analysis of reflective cracks in airfield pavements using a 3-D generalized finite element method. Road Materials and Pavement Design, 11 (2), pp. 459-477.

GILLESPIE, T.D. (1993) Effects of heavy-vehicle characteristics on pavement response and performance: Transportation Research Board.

HERNANDO, D. and DEL VAL, M.A. (2013) A Comprehensive Overview on Main Distress Mechanisms in Composite Pavements. International Journal of Pavement Research and Technology, 6 (6), pp. 737-744.

HUANG, Y.H., (1993) Pavement analysis and design. Englewood Cliffs, N.J.: Prentice Hall.

KAZIMIEROWICZ-FRANKOWSKA, K. (2008) Comparison of Stress and Strain States in Pavements with and without Reflective Cracks. Journal of Transportation Engineering, 134 (11), pp. 483-492.

KIM, Y.R. (2008) Modeling of asphalt concrete.

KOHALE, V. and LYTTON, R.L. (2000). Design of Asphalt Concrete Overlay to Mitigate Reflective Cracking.

KUO, C. and HSU, T. (2003) Traffic induced reflective cracking on pavements with geogridreinforced asphalt concrete overlay. In: Proceedings of the 82th Annual Meeting at the Transportation Research Board (CD-ROM).

LEE, Y.W. (2006). Discrete Element Modelling of Idealised Asphalt Mixture. 
LYTTON, R. L., F. L. TSAI, S. LEE, R. LUO, S. HU, and F. ZHOU. (2010). NCHRP Report 669: Models for Predicting Reflection Cracking for Hot-Mix Asphalt Overlays. Washington, D.C.: Transportation Research Board of the National Academies.

MAYHEW, H. and POTTER, J. (1986) Structural design and performance of lean concrete roads-bearing capacity of roads and airfields. Proceedings of the 2nd international conference, Plymouth, England, September 16-18, 1986. Publication of: WDM LIMITED.

MINHOTO, M., PAIS, J. and PEREIRA, P. (2008) Reflective cracking behavior for traffic and temperature effects. Pavement Cracking: Mechanisms, Modeling, Detection, Testing and Case Histories.

MINHOTO, M.J., PAIS, J.C. and PEREIRA, P.A. (2008) The temperature effect on the reflective cracking of asphalt overlays. Road Materials and Pavement Design, 9 (4), pp. 615-632.

MOLENAAR, A. and PU, B. (2008) Prediction of fatigue cracking in cement treated base courses. In: Proceedings of 6th RILEM International Conference on Cracking in Pavements, pp. 191-199.

MUKHTAR, M.T. and DEMPSEY, B.J. (1996). Interlayer Stress Absorbing Composite (ISAC) for Mitigating Reflection Cracking in Asphalt Concrete Overlays.

NATIONAL COOPERATIVE HIGHWAY RESEARCH PROGRAM, (NCHRP), (2010). Appendix R: Evaluation of available reflection cracking models. http://www.trb.org/Main/Blurbs/163988.aspx (last accessed 28/07/15)

NATIONAL RESEARCH COUNCIL (U.S.)., HIGHWAY RESEARCH BOARD. AMERICAN ASSOCIATION OF STATE HIGHWAY OFFICIALS., (1962) The AASHO road test. Report 7: Summary report. Washington: National Academy of Sciences-National Research Council.

NESNAS, K. et al. (2004) A model for top-down reflection cracking in composite pavements. In: Fifth international RILEM conference on reflective cracking in pavements: RILEM Publications SARL, pp. 409-416. 
NI, F. et al. (2008) Prediction model for shrinkage cracking spacing of cement stabilized base. In: Transportation Research Board 87th Annual Meeting.

OWUSU-ANTWI, E., KHAZANOVICH, L. and TITUS-GLOVER, L. (1998) Mechanistic-Based Model for Predicting Reflective Cracking in Asphalt Concrete-Overlaid Pavements. Transportation Research Record: Journal of the Transportation Research Board, 1629, pp. 234-241.

PAIS, J., MINHOTO, M. and SHATNAWI, S. (2012) Multi-cracks modeling in reflective cracking. In: 7th RILEM International Conference on Cracking in Pavements: Springer, pp. 441-452.

PAIS, J.C., PEREIRA, P.A. and MOHAMED, E.H. (2000) Prediction of existing reflective cracking potential of flexible pavements. In: Proceedings of 4th international RILEM conference, Ontario, pp. 155-164.

PAPAGIANNAKIS, A.T. et al. (1990) Impact of roughness-induced dynamic load on flexible pavement performance. In: Surface Characteristics of Roadways: International Research and Technologies: ASTM International.

PARMEGGIANI, G. (2012) Design of long life flexible composite and deep strength asphalt pavements. In: ARRB Conference, 25th, 2012, Perth, Western Australia, Australia.

PARRY, A. et al. (1999) Design and performance of flexible composite road pavements. In: Proceedings of the Institution of Civil Engineers-Transport: London: Published for the Institution of Civil Engineers by Thomas Telford Services, 1992-, pp. 9-16.

RAO, SHREENATH P., NATIONAL RESEARCH COUNCIL (U.S.)., TRANSPORTATION RESEARCH BOARD. SECOND STRATEGIC HIGHWAY RESEARCH PROGRAM (U.S.), (2013) Composite pavement systems. Volume 1, Volume 1. Washington, D.C.: Transportation Research Board.

ROBSON, J. (1979) Road surface description and vehicle response. International Journal of Vehicle Design, 1 (1), pp. 25-35.

SCARPAS, A. and DE BONDT, A. (1996) Finite elements simulation of reflective cracking in asphaltic overlays. Heron, 41 (1), 1996. 
SEEDS, S.B., MCCULLOUGH, B.F. and CARMICHAEL, R.F. (1985) Asphalt concrete overlay design procedure for Portland cement concrete pavements.

SEEDS, S.B., MCCULLOUGH, B.F. and CARMICHAEL, R.F. (1983). Arkansas Reflection Cracking Analysis and Overlay Design Procedure.

SHALABY, A., EL HALM, A. and EASA, S. (1996) Low-temperature stresses and fracture analysis of asphalt overlays. Transportation Research Record: Journal of the Transportation Research Board, (1539), pp. 132-139.

SOUSA, J.B. et al. (2002) Development of a mechanistic-empirical based overlay design method for reflective cracking. Transportation Research Board.

SOUSA, J.B., PAIS, J.C. and WAY, G.B. (2005) A Mechanistic-Empirical Based Overlay Design Method for Reflective Cracking. Road Materials and Pavement Design, 6 (3), pp. 339-363.

SWEATMAN, P. (1983) A study of dynamic wheel forces in axle group suspensions of heavy vehicles.

THOM, N. (2000) A simplified computer model for grid reinforced asphalt overlays. In: Fourth International RILEM Conference on Reflective Cracking in Pavements-Research in Practice: RILEM Publications SARL, pp. 37-46.

THOM, N., (2014) Principles of pavement engineering. London: Thomas Telford.

THOM, N. and CHEUNG, L. (1999) Relating in situ properties of cement-bound bases to their performance. Transportation Research Record: Journal of the Transportation Research Board, (1673), pp. 3-8.

THOM, N.H., CHOI, Y. and COLLOP, A.C. (2002). Top-down cracking, damage and hardening in practical flexible pavement design, 9th Int. Conf. on Asphalt Pavements, August 2002.

THOM, N.H., (1990)., Private correspondence Nottingham University, UK 
TOMPKINS, D., KHAZANOVICH, L. and DARTER, M.I. (2010) 2008 survey of European composite pavements.

TREVINO, M. et al. (2004). Applicability of Asphalt Concrete Overlays on Continuously Reinforced Concrete Pavements.

ULLIDTZ, P. et al. (2010) CalME, a Mechanistic-Empirical Program to Analyze and Design Flexible Pavement Rehabilitation. Transportation Research Record: Journal of the Transportation Research Board, 2153, pp. 143-152.

ULLIDTZ, P. (1993) Mathematical model of pavement performance under moving wheel load.

ULLIDTZ, P. (1979) A fundamental method for prediction of roughness, rutting and cracking of pavements. In: Association of Asphalt Paving Technologists Proceedings.

VANELSTRAETE, A. and FRANCKEN, L. (2004) Prevention of reflective cracking in pavements: CRC Press.

VON QUINTUS, H.L., FINN, F.N., HUDSON, W.R., and ROBERTS, F.L. (1979). Flexible and composite structures for premium pavements: Volume 1 and 2. FHWA-RD-81-154 FHWA-RD-81155. Federal Highway Administration, Washington, DC.

VON QUINTUS, H.L., (2016, August 17). TRB Webinar. Reflection Cracking Integration into Pavement ME Design. Retrieved from http://onlinepubs.trb.org/onlinepubs/webinars/160817.pdf

VON QUINTUS, H. et al. (1980) Flexible and composite structures for premium pavements. Vols.1 and 2, Report no. FHWA-RD, 80.

WALKER, D., and Cebon, D. (2011) The Metamorphosis of Long-Term Pavement Performance Traffic Data. TR News-Transportation Research Board 277.

WRIGHT, G. Deferring reflection cracks in asphalt overlays. HALLMARK CONFERENCE AND EVENTS, 2009, 25P (DAY 2, SESSION 4). 
WU, R. and HARVEY, J. (2012) Calibration of Asphalt Concrete Cracking Models for California Mechanistic-Empirical Design (CalME). In: SCARPAS, A. et al. (eds.) 7th RILEM International Conference on Cracking in Pavements. Dordrecht: Springer Netherlands, pp. 537-547.

YIN, H., BUTTLAR, W. and PAULINO, G.H. (2007) Simplified solution for periodic thermal discontinuities in asphalt overlays bonded to rigid pavements. Journal of Transportation Engineering, 133 (1), pp. 39-46.

YODER, ELDON J., WITCZAK, MATTHEW W., (1975) Principles of pavement design. New York: Wiley.

ZHOU, F., and SUN, L. (2000) Mechanistic analysis of reflective cracking and validation of field test. In: Fourth International RILEM Conference on Reflective Cracking in Pavements-Research in Practice: RILEM Publications SARL, pp. 81-91.

ZHOU, F., HU, S., HU, X., SCULliON, T., MIKHAIL, M., AND WALUBITA, L. (2010). Development, calibration, and verification of a new mechanistic-empirical reflective cracking model for HMA overlay thickness design and analysis. Journal of Transportation Engineering.

\section{Websites}

Infopave, https://infopave.fhwa.dot.gov/

FHWA, “LTPP Guide to Asphalt Temperature Prediction and Correction“, https://www.fhwa.dot.gov/publications/research/infrastructure/pavements/ltpp/fwdcd/ataf.cfm

FHWA, Advanced Methods for Using FWD Deflection-Time Data to Predict Pavement Performance, https://www.fhwa.dot.gov/publications/research/infrastructure/pavements/ltpp/97093/97093.pdf “LTPP Guide to Asphalt Temperature Prediction and Correction“, https://www.fhwa.dot.gov/publications/research/infrastructure/pavements/ltpp/fwdcd/ataf.cfm Advanced Methods for Using FWD Deflection-Time Data to Predict Pavement Performance, https://www.fhwa.dot.gov/publications/research/infrastructure/pavements/ltpp/97093/97093.pdf 


\section{Appendix A - Equations}

\section{Bottom-up cracking}

$$
\mathrm{P} \frac{\mathrm{d}^{2}}{4}+\frac{3}{2} \frac{\mathrm{EIP}}{\mathrm{kL}^{3}} \mathrm{~d}-\frac{3}{2} \frac{\mathrm{EIP}}{\mathrm{kL}^{2}}=0
$$

Where:

P: wheel load, $\mathrm{kN}$

$\mathrm{d}$ : debonded length, $\mathrm{m}$

E: asphalt elastic modulus, $\mathrm{MPa}$

I: moment of inertia of the asphalt beam, $\mathrm{m}^{4}$

$\mathrm{k}$ : subgrade of elastic modulus, $\mathrm{MN} / \mathrm{m}^{3}$

L: length of the slab, $m$

Tensile stress in the region of the crack tip:

$$
\sigma_{2}=\frac{3}{2} \frac{M}{d_{1}^{2}}
$$

Moment if radius of wheel patch is greater than debonded length:

$$
M=\frac{P}{4}(2 d-r)
$$

Or if the radius is smaller than debonded length:

$$
\mathrm{M}=\frac{\mathrm{Pd}^{2}}{4 \mathrm{r}}
$$

The equivalent maximum tensile strain at the crack will be:

$$
\varepsilon_{\mathrm{t}}=\frac{\sigma_{2}}{\mathrm{E}}
$$

Crack propagation rate for bottom-up cracking:

$$
\frac{\mathrm{dc}}{\mathrm{dN}}=A \varepsilon_{\mathrm{t}}^{\mathrm{n}}
$$

Where: A and $\mathrm{n}$ are constants 
Crack length development from bottom-up:

$$
\mathrm{C}_{\mathrm{bu}}=\frac{\mathrm{dc}}{\mathrm{dN}} \times \mathrm{N}
$$

Destressed length:

$$
t=\sqrt{\left(8 C_{b u}^{2}(1+v)\left(\frac{h}{4 C_{b u}}-\frac{1}{6}\right)\right)}
$$

where v is Poisson's ratio.

Effective modulus of the asphalt (Eeff) after appearance of bottom-up crack:

$$
E_{e f f}=\frac{E}{\left[\frac{L-2 t}{L h^{3}}-\frac{t}{L C_{b u} h^{2}}+\frac{t}{L C_{b u}\left(h-C_{b u}\right)^{2}}\right] h^{3}}
$$

\section{Top-down cracking}

Stress at the tip of the crack would be:

$$
\sigma_{1}=\frac{3}{2} \frac{M}{d_{1}^{2}}
$$

Moment in top-down cracking:

$$
\mathrm{M}=\frac{\mathrm{EI}}{\mathrm{d}}\left(\frac{6 \delta_{1}}{\mathrm{~d}}-4 \Sigma \theta_{1}\right)
$$

Where $\delta_{1}$ and $\theta_{1}$ are shown in Figures 11 and 13 in the main text in Chapter 2 (2.2.2), $\delta_{1}$ is the differential deflection, slab $B$ less slab $A$ and $\theta_{1}$ is the total angle created at location $1_{\mathrm{a}}$ and $1_{\mathrm{b}}\left(\theta_{1}=\right.$ $\left.\theta_{\alpha}+\theta_{\beta}\right)$

The tensile strain at crack would be given by:

$$
\varepsilon_{\text {crack }}=\frac{\sigma_{1}}{\mathrm{E}}
$$


Approximation to estimate surface shear strain due to a wheel load. The suggested equation for modelling the surface profile is given by:

$$
y=\frac{p h}{E} e^{\left[\left(\frac{x}{h}\right) \sqrt{6(1+v)}\right]}
$$

And:

$$
\frac{d y}{d x}=\frac{p}{E} \sqrt{6(1+v)}
$$

Where:

P: Wheel load per m. width $(\mathrm{kN} / \mathrm{m})$

h: Asphalt thickness (m)

v: Poisson's ratio

E: Asphalt elastic modulus

But it is assumed that the surface shear strain $\left(\gamma_{\mathrm{s}}\right)$ at $\mathrm{x}=0$ is:

$$
\frac{\mathrm{dy}}{\mathrm{dx}}=\gamma_{\mathrm{s}}
$$

In addition, a component of shear stress is generated by bending of the asphalt layer which is given by and the equivalent bending shear strain $(\gamma \mathrm{b})$ is given by:

$$
\gamma_{\mathrm{b}}=\frac{\mathrm{F}}{\mathrm{GH}_{\mathrm{eff}}}
$$

Where:

F: Shear force in asphalt. The detailed procedure for calculating F can be found in Thom (1990).

G: Asphalt shear modulus

Heff: Effective asphalt thickness (uncracked thickness)

The total shear strain would be:

$$
\gamma=\gamma_{\mathrm{s}}+\gamma_{\mathrm{b}}
$$

So, the total strain (Mohr circle) is given by:

$$
\varepsilon_{\mathrm{t}}=\frac{\varepsilon_{\text {crack }}}{2} \pm \sqrt{\left(\frac{\varepsilon_{\text {crack }}}{2}\right)^{2}+\left(\frac{\gamma}{2}\right)^{2}}
$$


And the direction of the strain:

$$
\tan 2 \mathrm{a}=\frac{\gamma}{\varepsilon_{\text {crack }}}
$$

Crack propagation rate for top-down cracking:

$$
\frac{\mathrm{dc}}{\mathrm{dN}}=\mathrm{A} \varepsilon_{\mathrm{t}}^{\mathrm{n}} \cos \mathrm{a}
$$

A, n: constants

Crack length development from top-down:

$$
\mathrm{C}_{\mathrm{td}}=\frac{\mathrm{dc}}{\mathrm{dN}} \times \mathrm{N}
$$

Where $\frac{\mathrm{dc}}{\mathrm{dN}}$ the crack propagation rate, $\mathrm{N}$ the number of wheel loads and $\mathrm{C}_{\mathrm{td}}$ the length of the crack development from bottom to up.

\section{Thermal reflective cracking}

Conversion of air temperature to pavement temperature:

$$
\mathrm{T}_{\text {pav }}=\mathrm{T}_{\mathrm{air}} \times\left(1+\frac{76.2}{\mathrm{~h}+304.8}\right)-\frac{84.7}{\mathrm{~h}+304.8+3.3}
$$

Where $\mathrm{h}$ is the asphalt thickness in $\mathrm{mm}$.

From the heat flow equation:

$$
\frac{\mathrm{dT}}{\mathrm{dt}}=\frac{\mathrm{k}}{\mathrm{C} \rho} \frac{\mathrm{d}^{2} \mathrm{~T}}{\mathrm{dh}^{2}}
$$

Where:

T: Temperature

h: Asphalt thickness

$\mathrm{k}$ : Thermal conductivity (W/mk)

C: Specific heat capacity $(\mid \mathrm{J} / \mathrm{kgK})$ 
$\rho:$ Density $\left(\mathrm{kg} / \mathrm{m}^{3}\right)$

Solving this differential equation gives the solution with which the temperature distribution of the pavement layers is estimated (Thom, 2014):

$$
\mathrm{T}=\mathrm{T}_{\text {mean }}+\mathrm{T}_{\mathrm{amplitude}} \mathrm{e}^{-\mathrm{Ah}} \sin (\mathrm{Ah}+\omega \mathrm{t})
$$

$\mathrm{T}_{\text {mean: }}$ Mean daily temperature $\left({ }^{\circ} \mathrm{C}\right)$

Tamplitude: Amplitude of daily temperature $\left({ }^{\circ} \mathrm{C}\right)$

h: Asphalt thickness (mm)

A: factor $A=\frac{C \rho \omega}{2 k}$

$\omega=\frac{2 \pi}{T}$ where $T=24 h$

Layer expansion due to temperature difference:

$$
\Delta \mathrm{x}=\alpha \mathrm{L} \Delta \mathrm{T}
$$

Expansion for base layer:

$$
\text { Expansion }_{\text {base }}=\mathrm{a}_{\text {base }} \times \mathrm{L} \times \Delta T
$$

abase: Thermal expansion coefficient of base layer

Viscous strain:

$$
\dot{\varepsilon}=\frac{\dot{\delta}}{\sqrt{\frac{\eta_{\mathrm{tc}}}{\eta_{\text {mix }} h t}}+d}
$$

Where $\dot{\delta}$ : movement rate of point of slab 1

$\eta_{\mathrm{tc}}$ : viscosity of tack coat

$\eta_{\text {mix: viscosity of mixture }}$

h: asphalt thickness 

t: tack coat thickness
d: debonded length between asphalt and rigid base

Strain due to the asphalt expansion during every hour would be:

$$
\text { Asphalt thermal strain }=\left(\mathrm{T}_{\mathrm{asph}(\text { new })}-\mathrm{T}_{\mathrm{asph}(\text { previous })}\right) \times \mathrm{a}_{\mathrm{asph}}
$$

aasph: Thermal expansion coefficient for asphalt layer

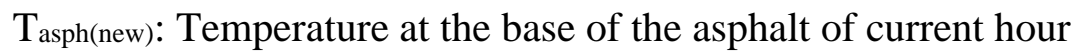

$\mathrm{T}_{\text {asph(previous): }}$ Temperature at the base of the asphalt of previous hour

Total thermal strain:

$$
\varepsilon=\text { Total Strain }=\text { Viscous strain }+ \text { Asphalt thermal strain }
$$

Crack propagation rate for thermal cracking:

$$
\frac{\mathrm{dc}}{\mathrm{dN}}=A \varepsilon^{\mathrm{n}}
$$




\section{Appendix B - Matlab code}

\section{B.1 Reflective cracking mechanical model}

$\%$ Olcrack 2019

\% Evangelia Manola

clear all

$\% \%$ Notes

$\% \%$ Time increment for iri update and new crack development is different

$\% \%$ if same: then iri_time=1

$\% \%$ choose between either dynamic OR static loading

$\% \%$ all vehicle models are included

\%\% slablength (crack spacing) defines locations where forces are estimated to develop a crack

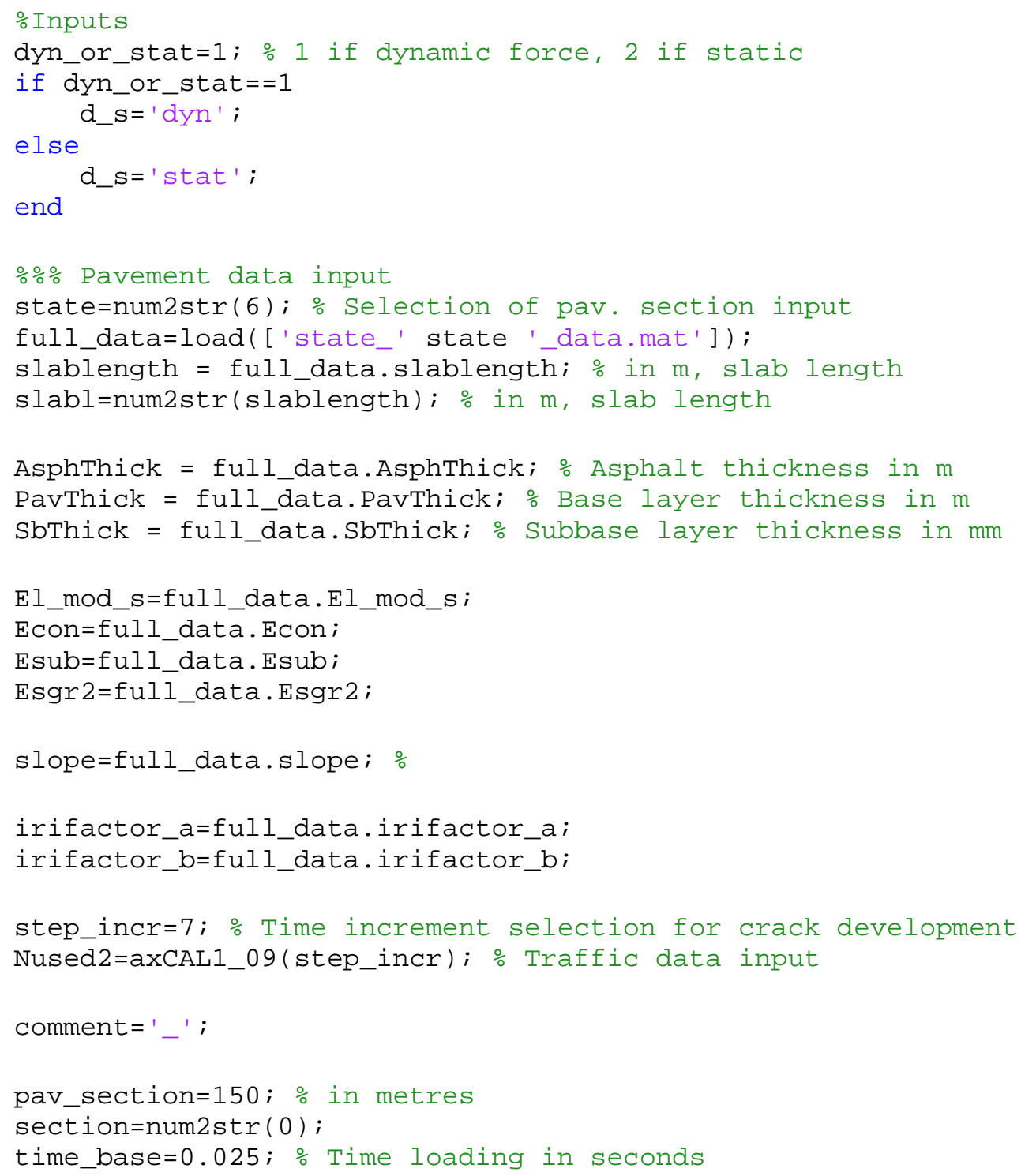




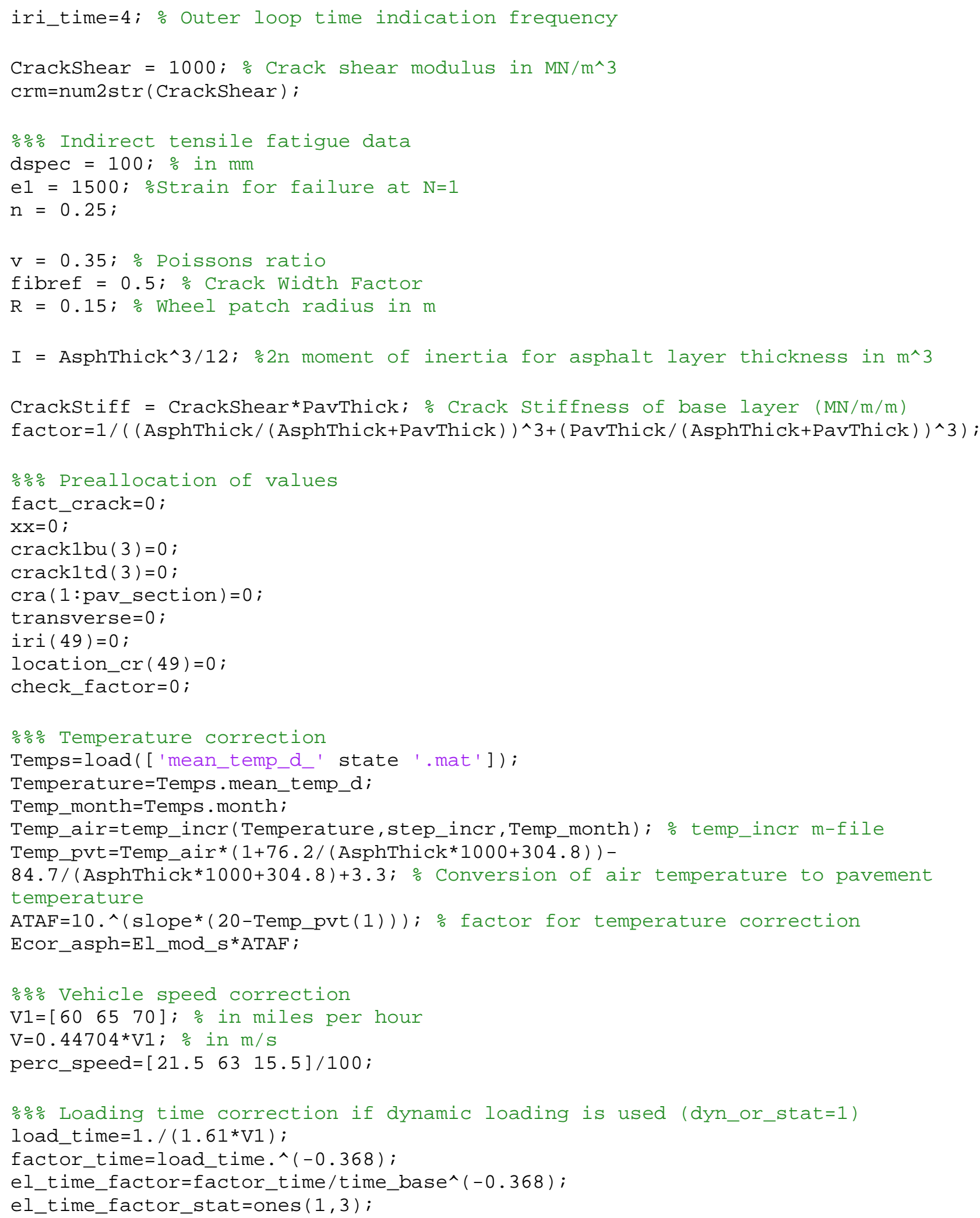




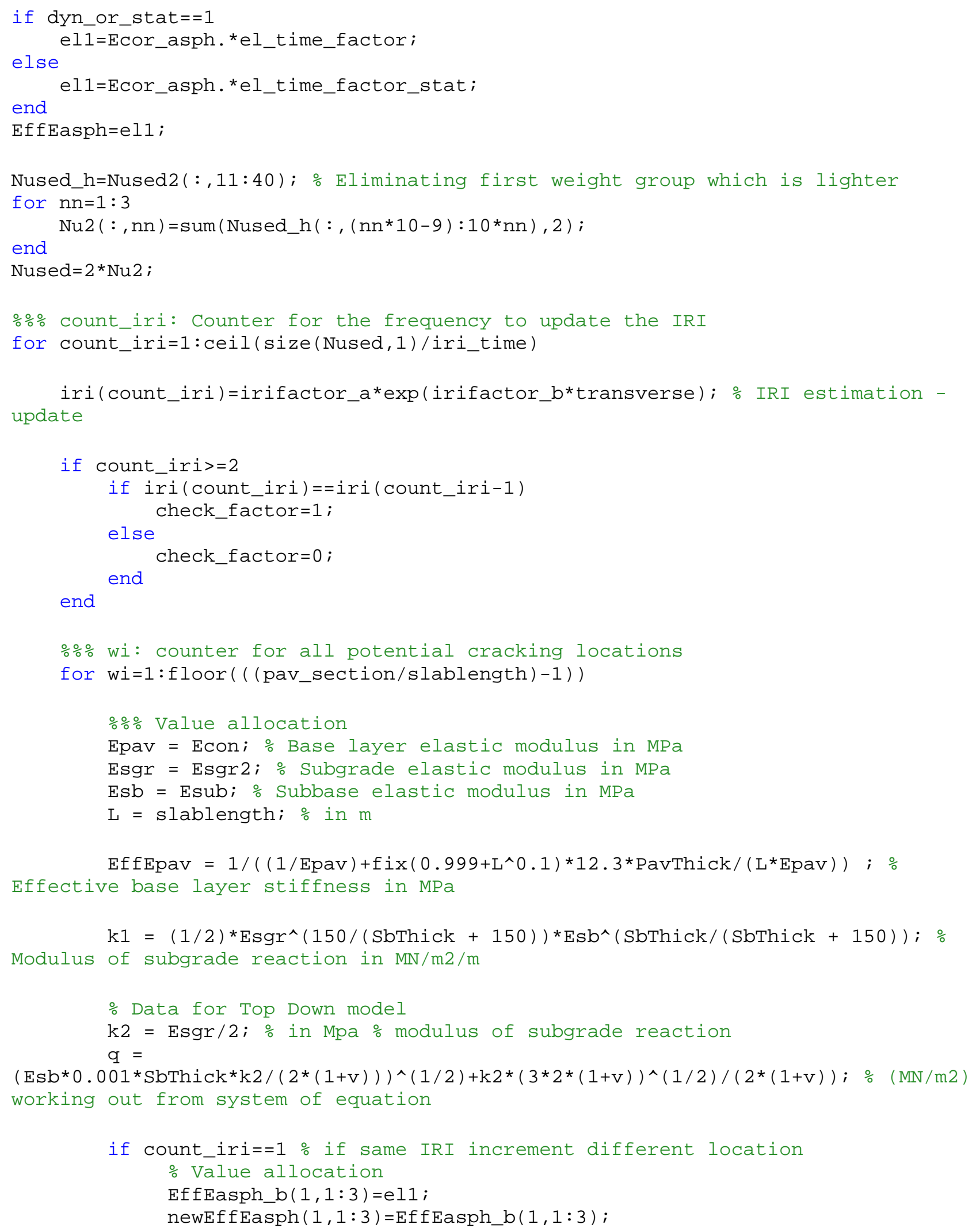




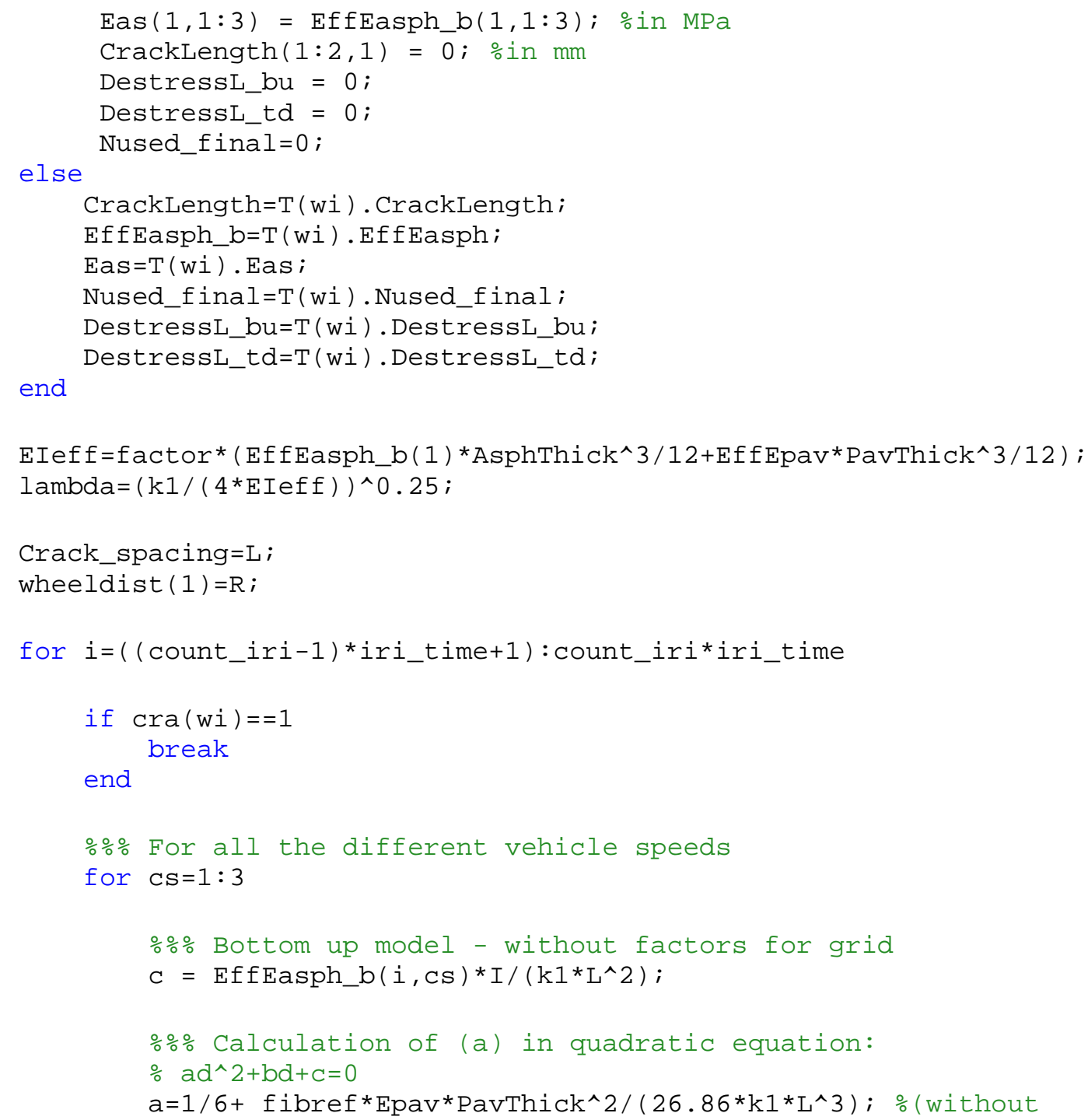


end

F=FORCE_STAT $(:,:, C S)$;

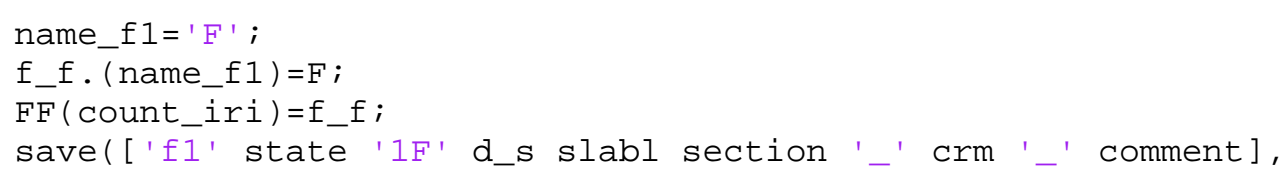

$\operatorname{Thick}(i)]=$

Load=F(wi, : );

Load_2D=Load*lambda/2;

for $w=1:$ length $($ Load $)$

$\% \%$ Calculation of crackRate for bottom-up cracking

[moment_bu( $i, w)$, CrackRatebu $(i, w)$, Tensstrain_bu( $i, w)$,

bottomUp_cracking(CrackLength(1,i),CrackLength(2,i), Load_2D(w),Load(w), DebLength (wi, i, cs) , R, EffEpav, PavThick, k1, SbThick, Esb, AsphThick, v, EffEasph(1, cs) , dspec, e1, $n, \operatorname{Eas}(i, c s))$;

Critical Surface shear

$\% \%$ Top-down model

$\% \%$ Calculation of Critical moment, Critical Bending Shear,

[Critical_moment_td(i), Critical_BendShear(i),

Critical_SurfShear $(i), M 1 \_v e c t o r, f 1 \_v e c t o r$, index_td, $x(i,:)$,

remain_thick(i)]=topDown_cracking(Crack_spacing, Load $(1, w)$, Load_2D(1,w),EffEasph( $1, c s)$, moment_bu( $i, w)$, AsphThick, CrackLength $(2, i), R$, DestressL_td, $i, k 2$, Crackstiff, I $, q$, CrackLength $(1, i))$;

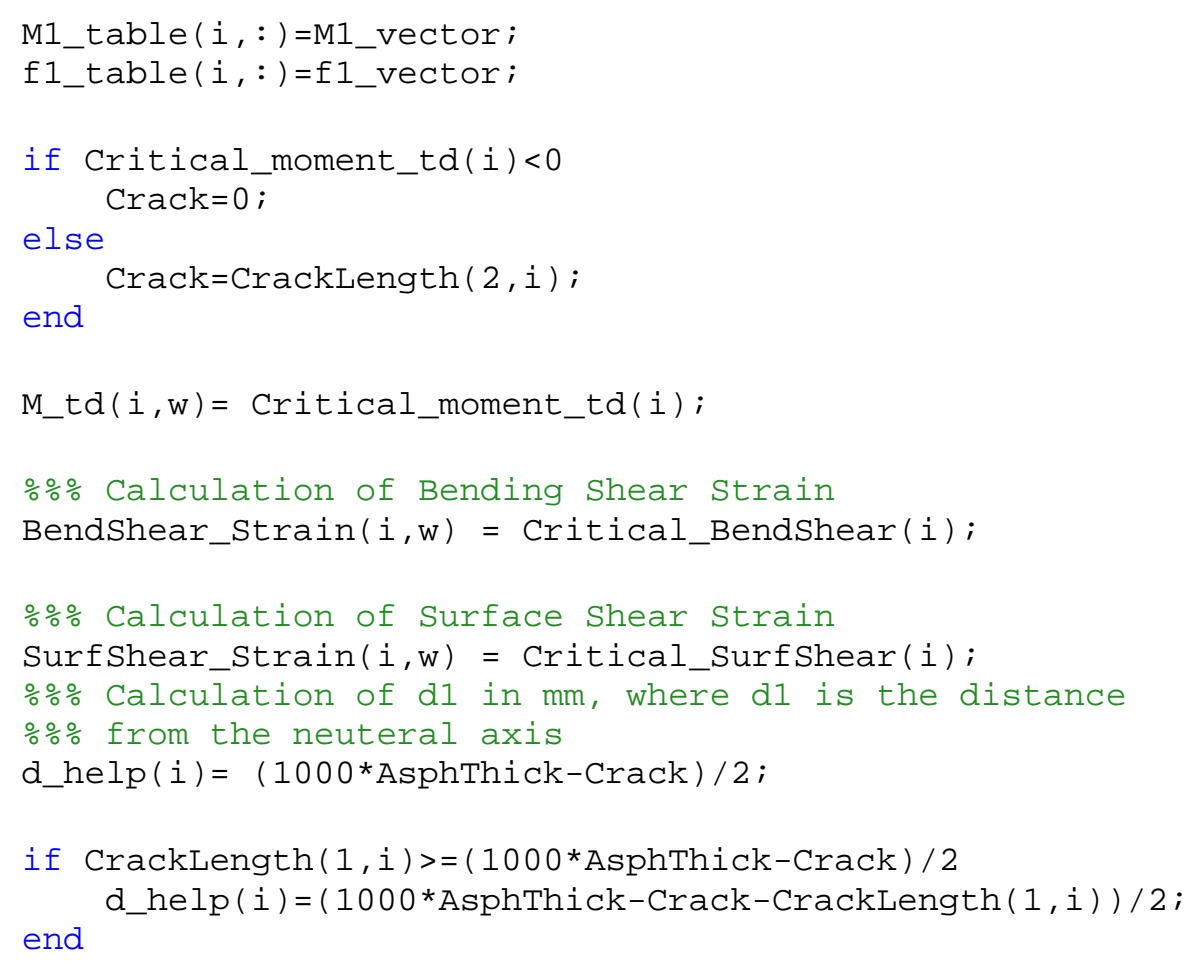




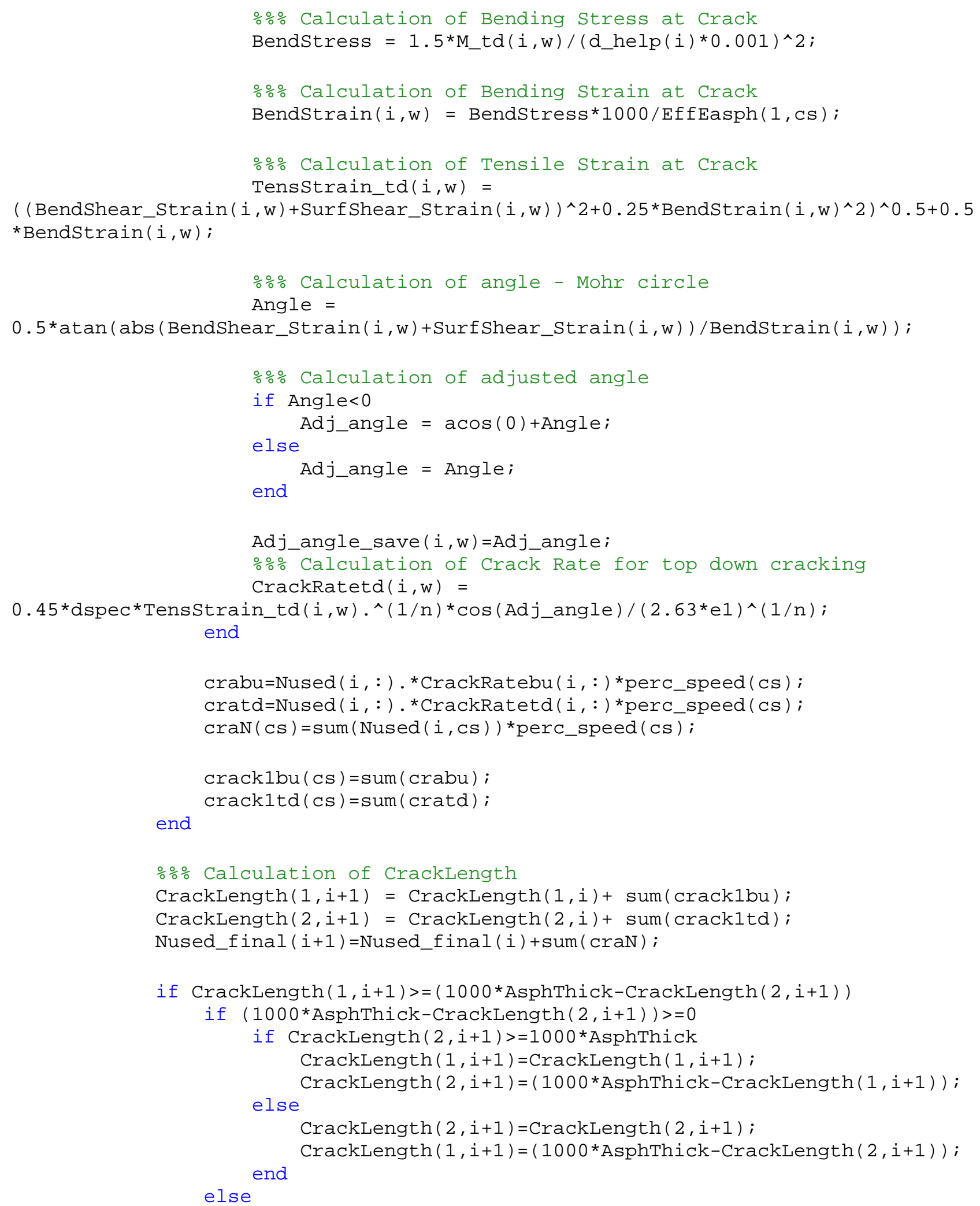




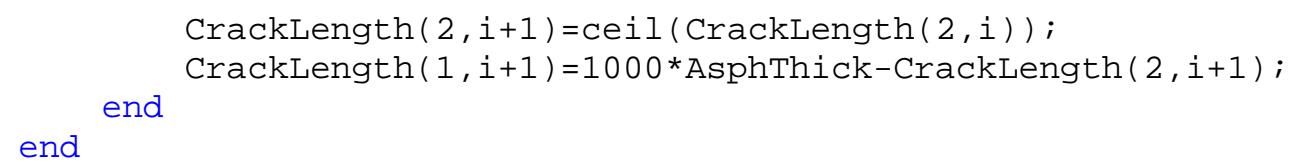

S. CrackLength $=$ CrackLength;

S. EffEasph=EffEasph;

S.DestressL_bu=DestressL_bu;

S.DestressL_td=DestressL_td;

S.Nused_final=Nused_final;

S. Eas=Eas ; 


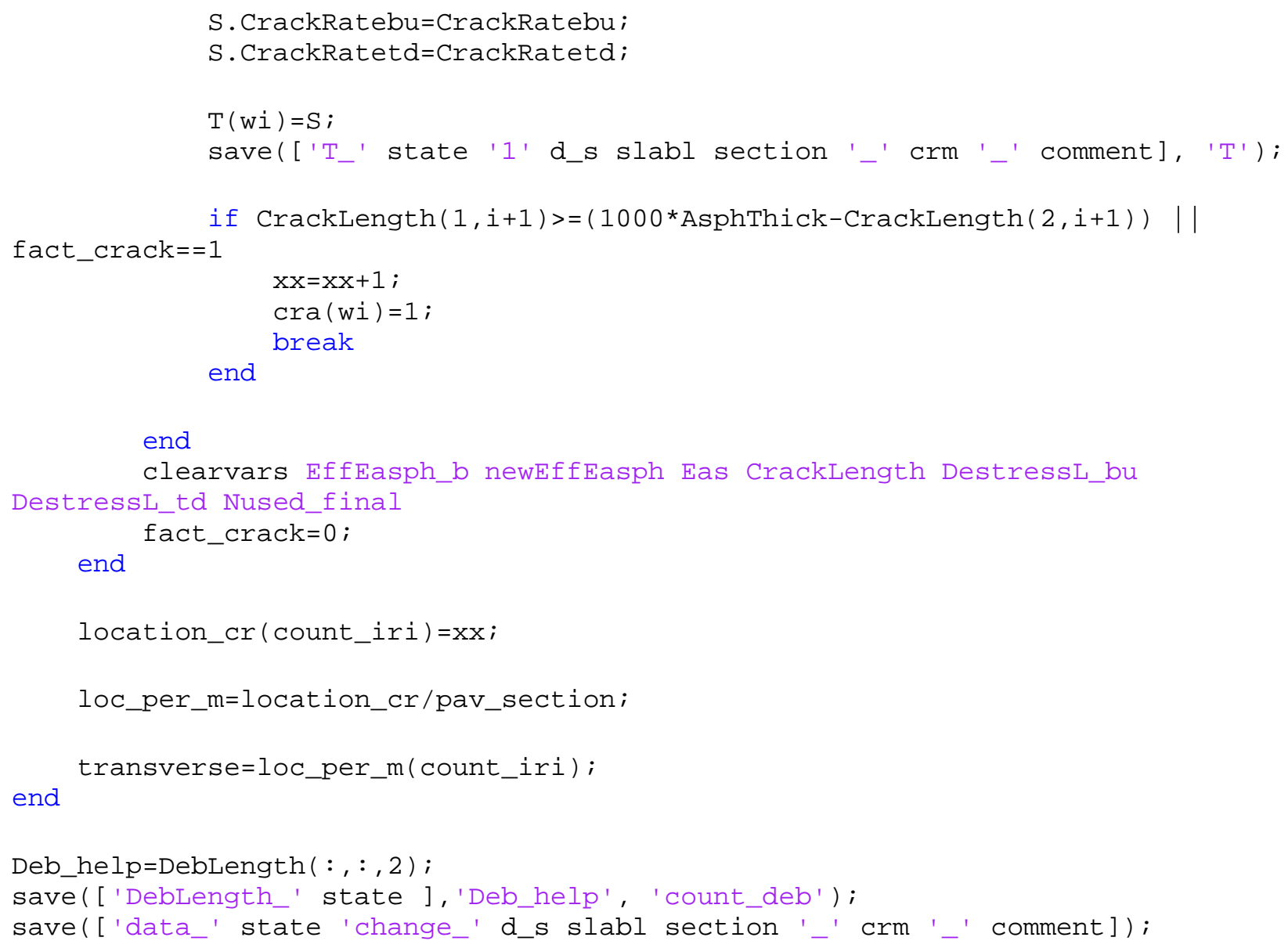

\section{B.2 Reflective cracking thermal model}

$\%$ Thermcrack 2019

\% Evangelia Manola

clear all

\%Inputs

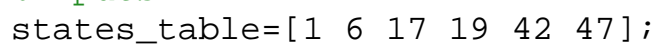

for st=1: length (states_table)

state=num2str (states_table $($ st $))$;

comment $={ }^{\prime}-$ all' ;

full_data=load(['state_'state '_data.mat']);

Spacing = full_data.slablength; \% crack spacing in $\mathrm{m}$ Dasph = full_data.AsphThick; \% Asphalt thickness in $\mathrm{m}$

PavThick = full_data.PavThick; \% Base layer thickness in $\mathrm{m}$ 


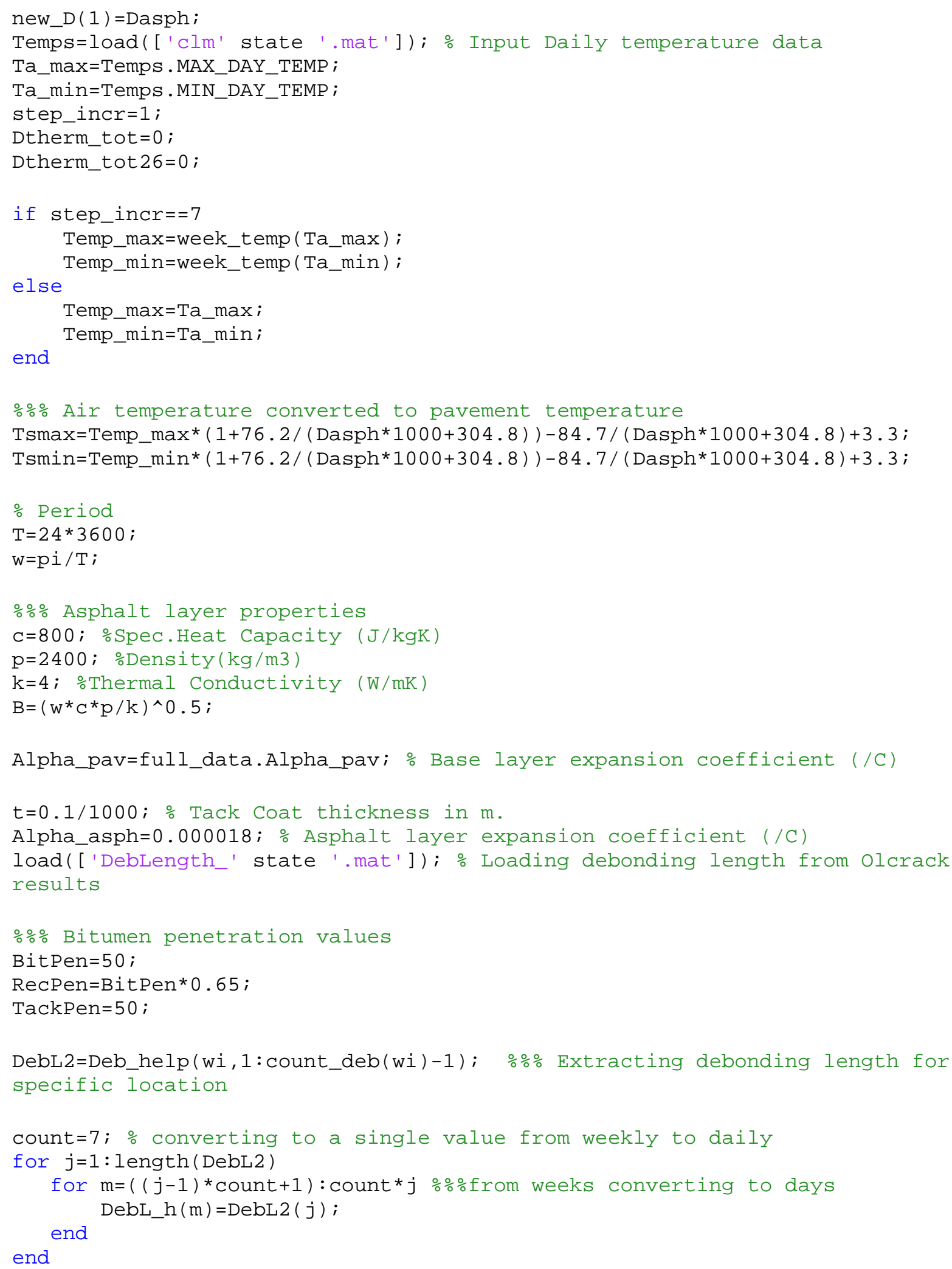




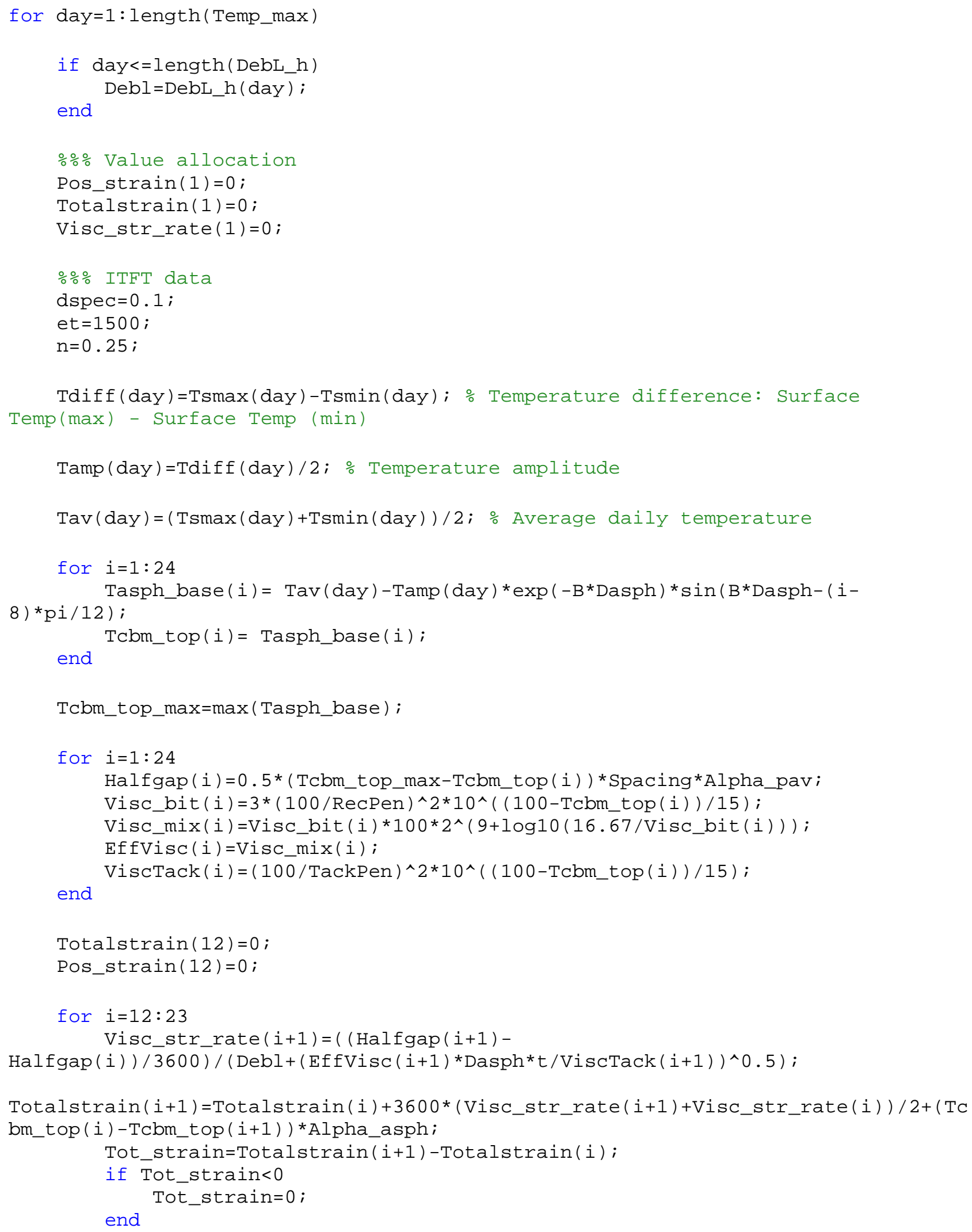


end

Pos_strain $(i+1)=$ Pos_strain $(i)+$ Tot_strain;

for $i=1: 6$

if $i==1$

Visc_str_rate $(i)=($ (Halfgap $(i)-$

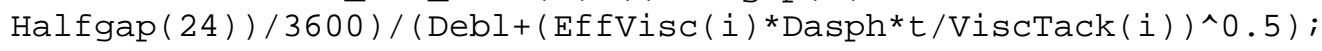

Totalstrain $(i)=$ Totalstrain (24) +3600* (Visc_str_rate $\left.(i)+V i s c \_s t r \_r a t e(24)\right) / 2+($ Tcbm _top(24)-Tcbm_top(i))*Alpha_asph;

Tot_strain=Totalstrain(i) - Totalstrain(24);

if Tot_strain<0

end

else

Pos_strain(i)=Pos_strain(24)+Tot_strain;

Visc_str_rate $(i)=((\operatorname{Halfgap}(i)-\operatorname{Halfgap}(i-$

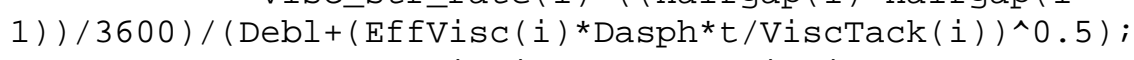

Totalstrain $(i)=$ Totalstrain $(i-$

$1)+3600 *\left(V i s c \_s t r \_r a t e(i)+V i s c \_s t r \_r a t e(i-1)\right) / 2+\left(T c b m \_t o p(i-1)-\right.$

Tcbm_top(i))*Alpha_asph;

Tot_strain=Totalstrain(i) - Totalstrain(i-1);

if Tot_strain<0

end

Tot_strain=0;

end

Pos_strain $(i)=$ Pos_strain $(i-1)+$ Tot_strain;

end

Et_max $($ day $)=\max ($ Pos_strain $) * 1000000 ; \%$ Maximum daiy thermal strain

$A=0.225^{*}$ dspec $/\left(2.63^{*} \text { et }\right)^{\wedge}(1 / \mathrm{n})$;

$\mathrm{N} 1=\operatorname{Dasph}^{\wedge}(1+1 / \mathrm{n}) /\left((1+1 / \mathrm{n}){ }^{*} \mathrm{~A}^{*}\left(\text { Dasph . }{ }^{*} \mathrm{Et} \_\max (\text { day })\right)^{\wedge}(1 / \mathrm{n})\right)$;

$N($ day $)=$ floor $(N 1)$;

$\mathrm{N} 1 \_26$ (day) $=\mathrm{N}($ day $) / 26 ; \%$ Calibration years

N_26 (day) $=$ N1_26 (day) ${ }^{*} 365$; \% convert to days

$\% \%$ Thermal damage

Dtherm (day) $=1 / \mathrm{N}$ (day);

Dtherm_26(day) $=1 /$ N_26 (day) ;

Dtherm_tot=Dtherm_tot+Dtherm(day);

Dtherm_tot26=Dtherm_tot26+Dtherm_26(day)；

if Dtherm_tot26>=1

end break

end 


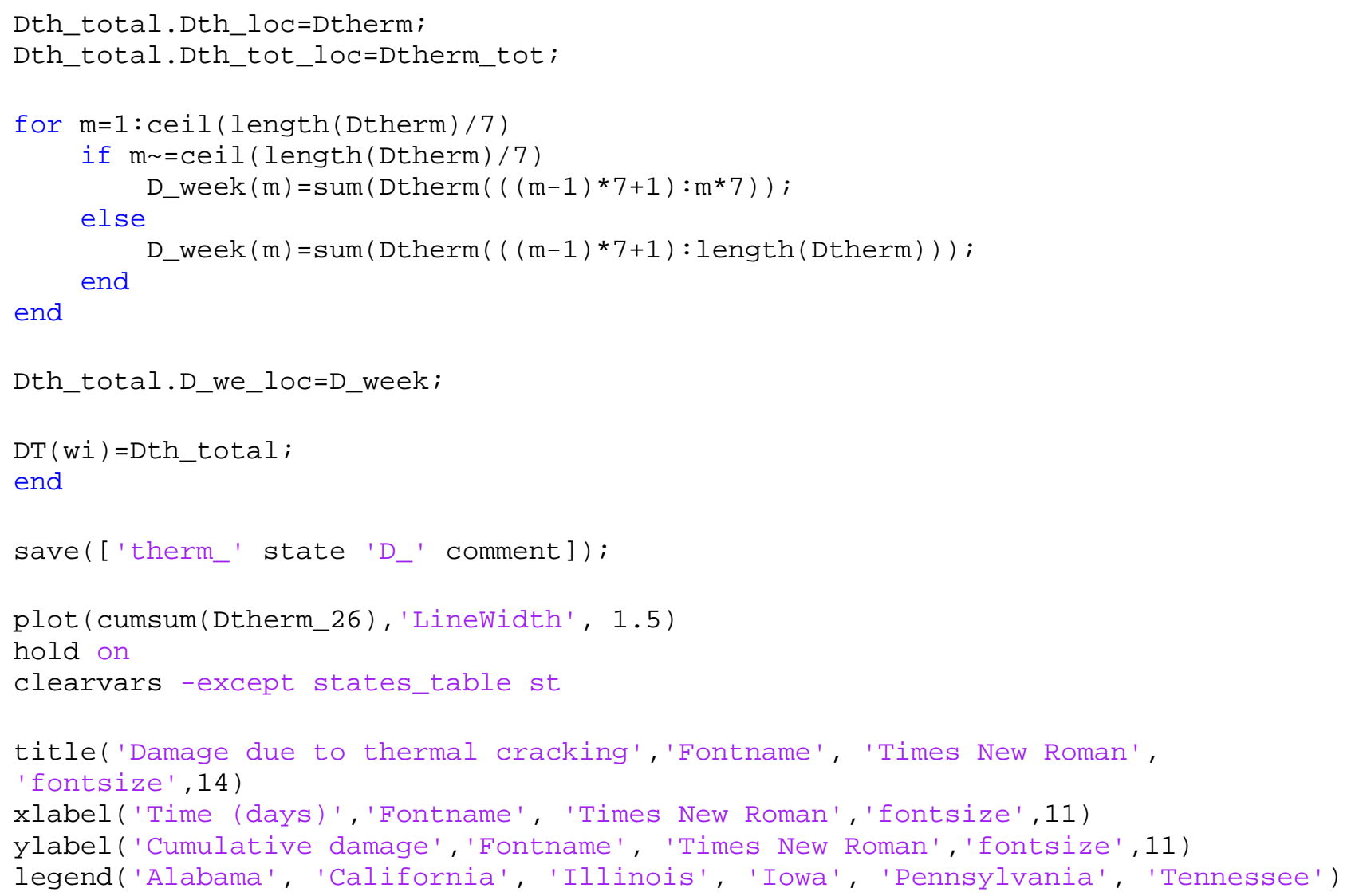

\section{B.3 Bottom-up cracking sub-model}

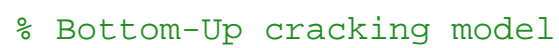




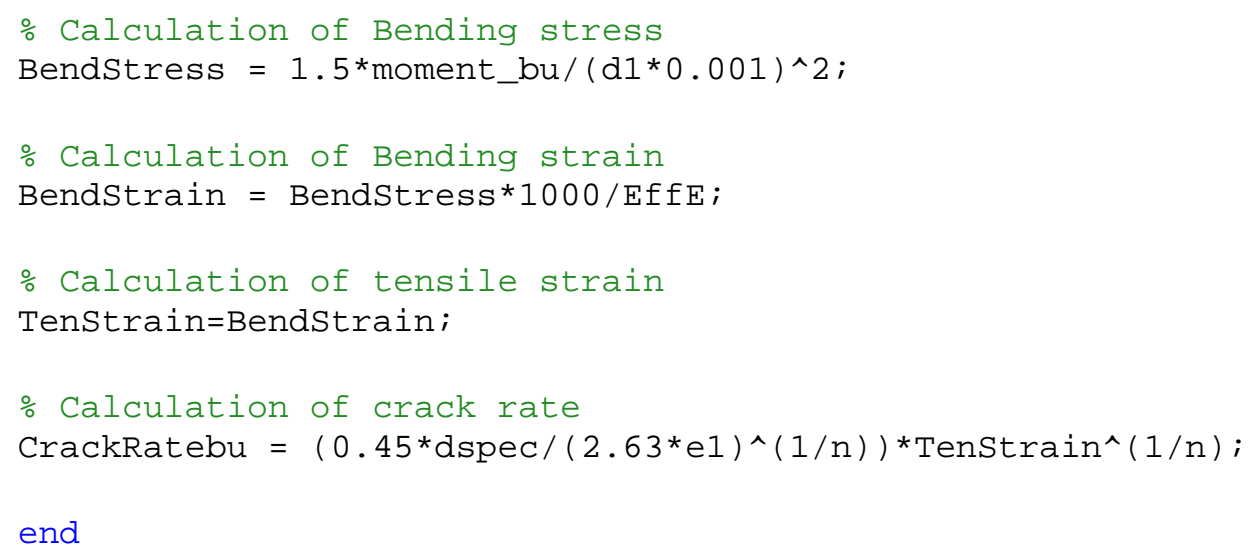

\section{B.4 Top-down cracking sub-model}

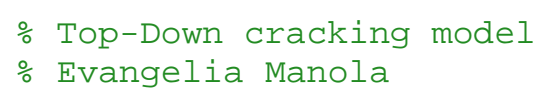

function[Critical_moment_td, Critical_BendShear, Critical_SurfShear, M1_vector,f1_vector, index_td, x2, remain_thick]=topDown_cracking (Crack_spacing, Load, Load2,EffE, moment_bu, AsphThick , CrackLength_td, R, DestressL_td, i, k2, CrackStiff, I, q, CrackLength_bu)

wheeldist $(1)=\mathrm{R}$;

incr_dist $=($ Crack_spacing $/ 2-$ wheeldist $(1)) / 20$;

Total_et $(1)=0$;

max_et $=$ Total_et $(1)$;

maxposition=1;

$\mathrm{v}=0.35 ; \%$ Poissons ratio

for $j=1: 20$

$$
\begin{aligned}
& \text { if CrackLength_td }=0 \\
& \text { EffEasph }=\text { EffE } /(((2 * \text { wheeldist }(j)-
\end{aligned}
$$

$2{ }^{*} \odot . \odot \odot 1^{*}$ DestressL_td $) /(2 *$ wheeldist $(j) * A s p h T h i c k \wedge 3)-$

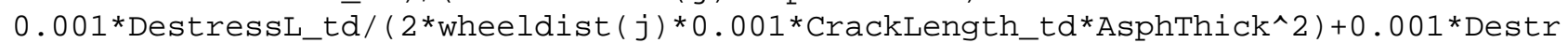
essL_td/(2*wheeldist $(j) * 0.001{ }^{*}$ CrackLength_td* (AsphThick -

$\odot .0 \odot 1^{*}$ CrackLength_td $\left.\left.)^{\wedge} 2\right)\right)^{*}$ AsphThick^3);

else

end

EffEasph = EffE;

if $j==1$ end

AsphShear_Mod $=$ EffEasph $/\left(2^{*}(1+\mathrm{v})\right) ; \%$ in MPa \% asphalt shear modulus

$\left[\mathrm{M} 1_{-}, \mathrm{f} 1_{-}, \mathrm{x}\right]=$

solve_TopDown(Crack_spacing, k2, Load2, wheeldist ( j) ,CrackStiff,EffEasph, I, q, AsphSh ear_Mod, AsphThick);

$M 1 \_\operatorname{vector}(j)=M 1 \_;$ 


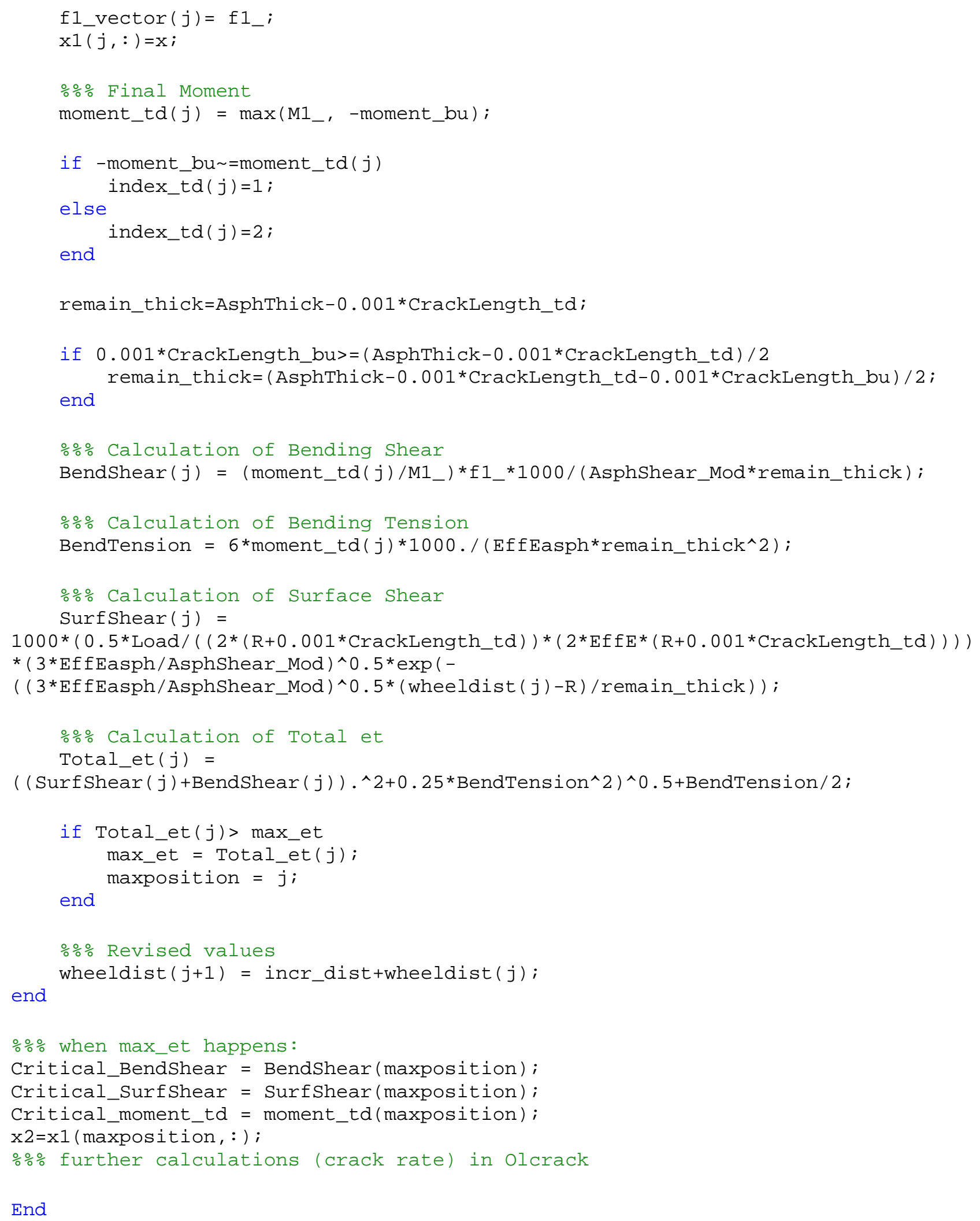




\section{B.5 Top-down cracking sub-model (2) (solving system)}

\% System of Equations Top-Down cracking model

\% Evangelia Manola

function $\left[\mathrm{M} 1_{-}, \mathrm{f} 1_{-}, \mathrm{x}\right]=$ solve_TopDown(L, k2, Load2, wheeldist, CrackStiff,EffEasph, I, q,AsphShear_Mod,AsphThi $\mathrm{ck}$ )

\%\% Solving system of 14 equations for top-down cracking

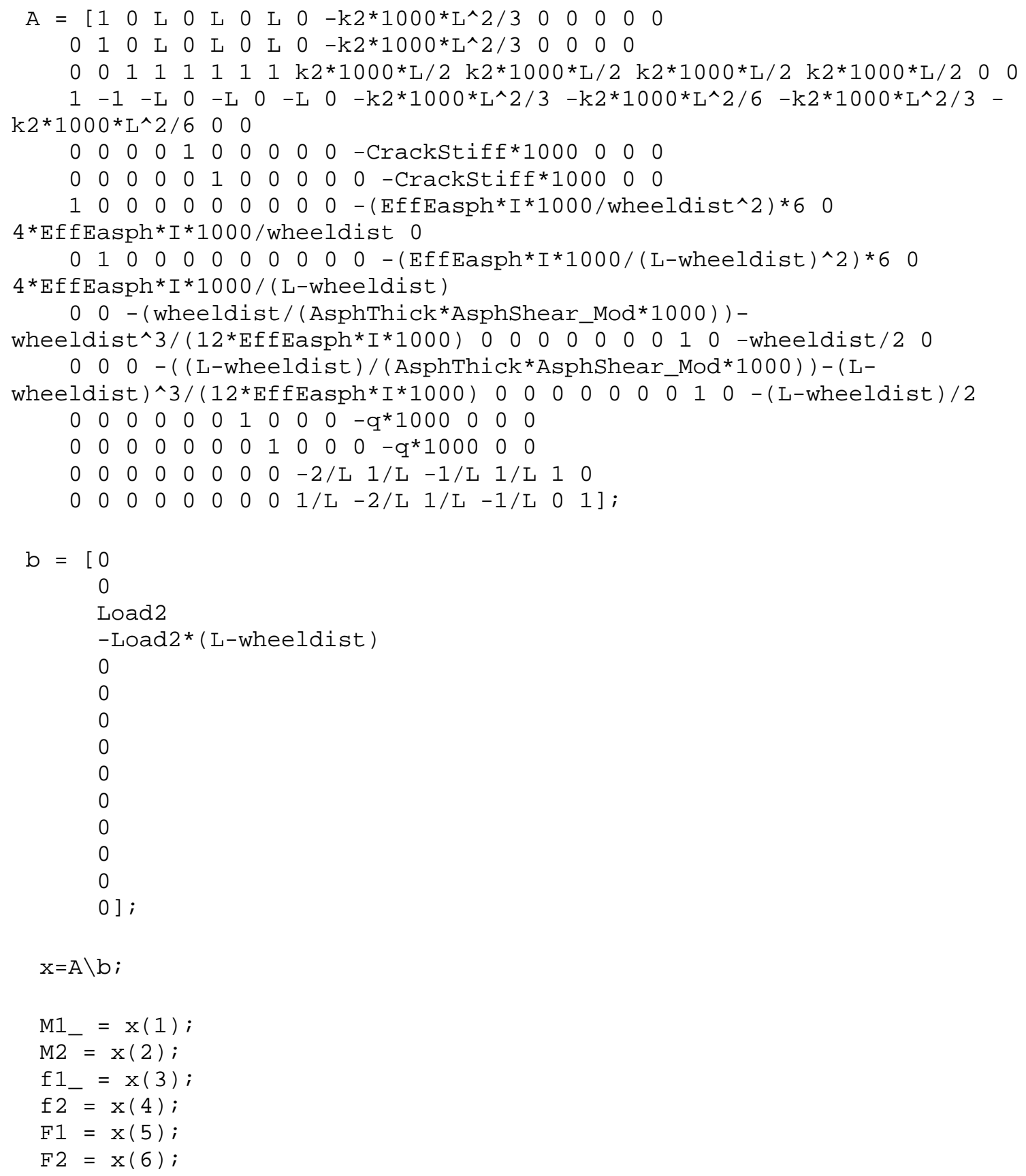




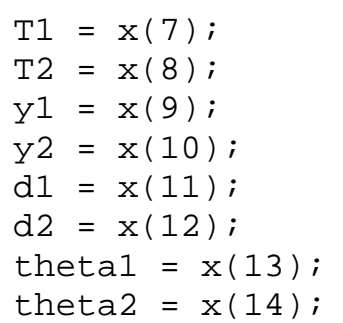

end

\section{B.6 Vehicle dynamic model}

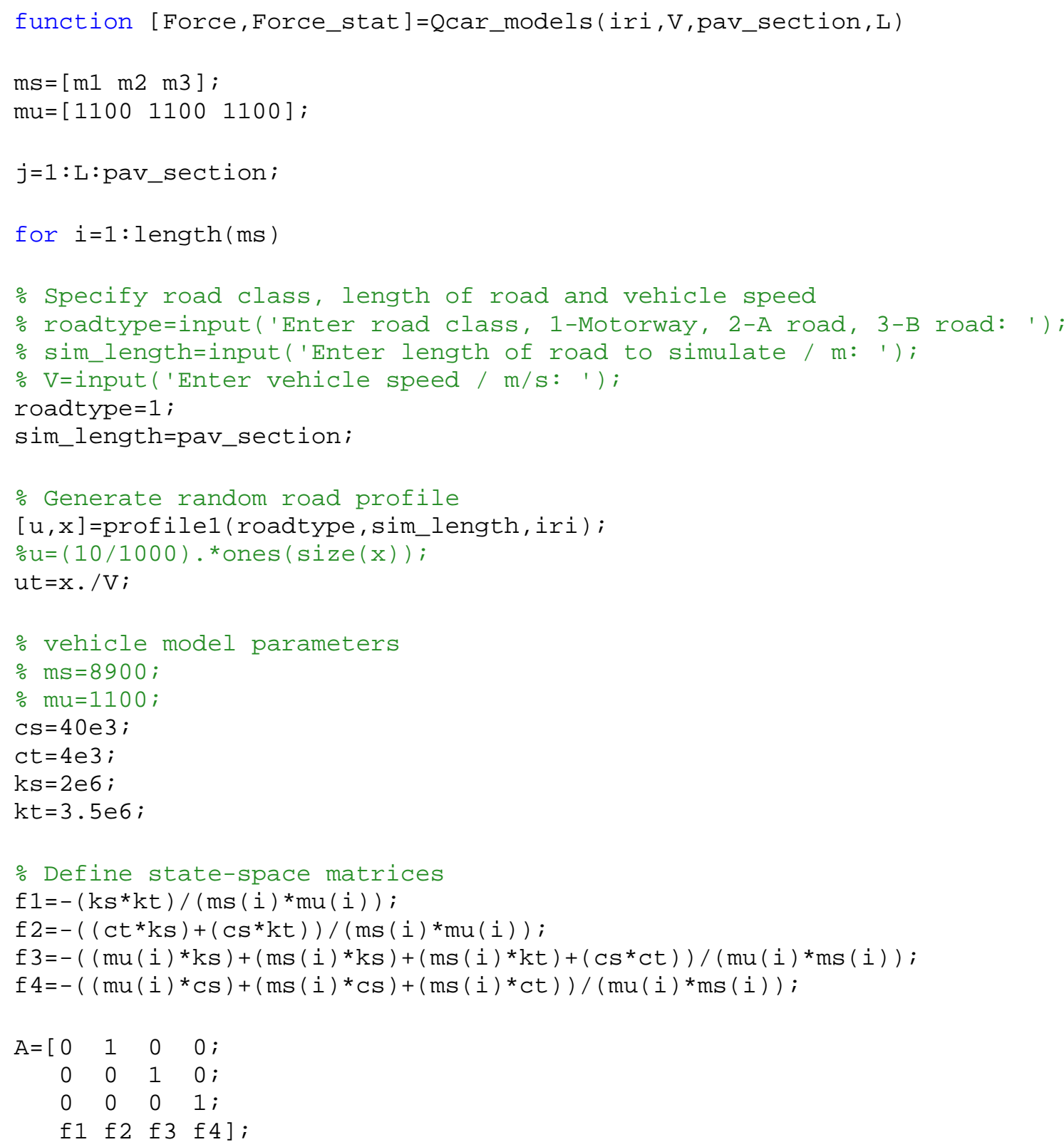




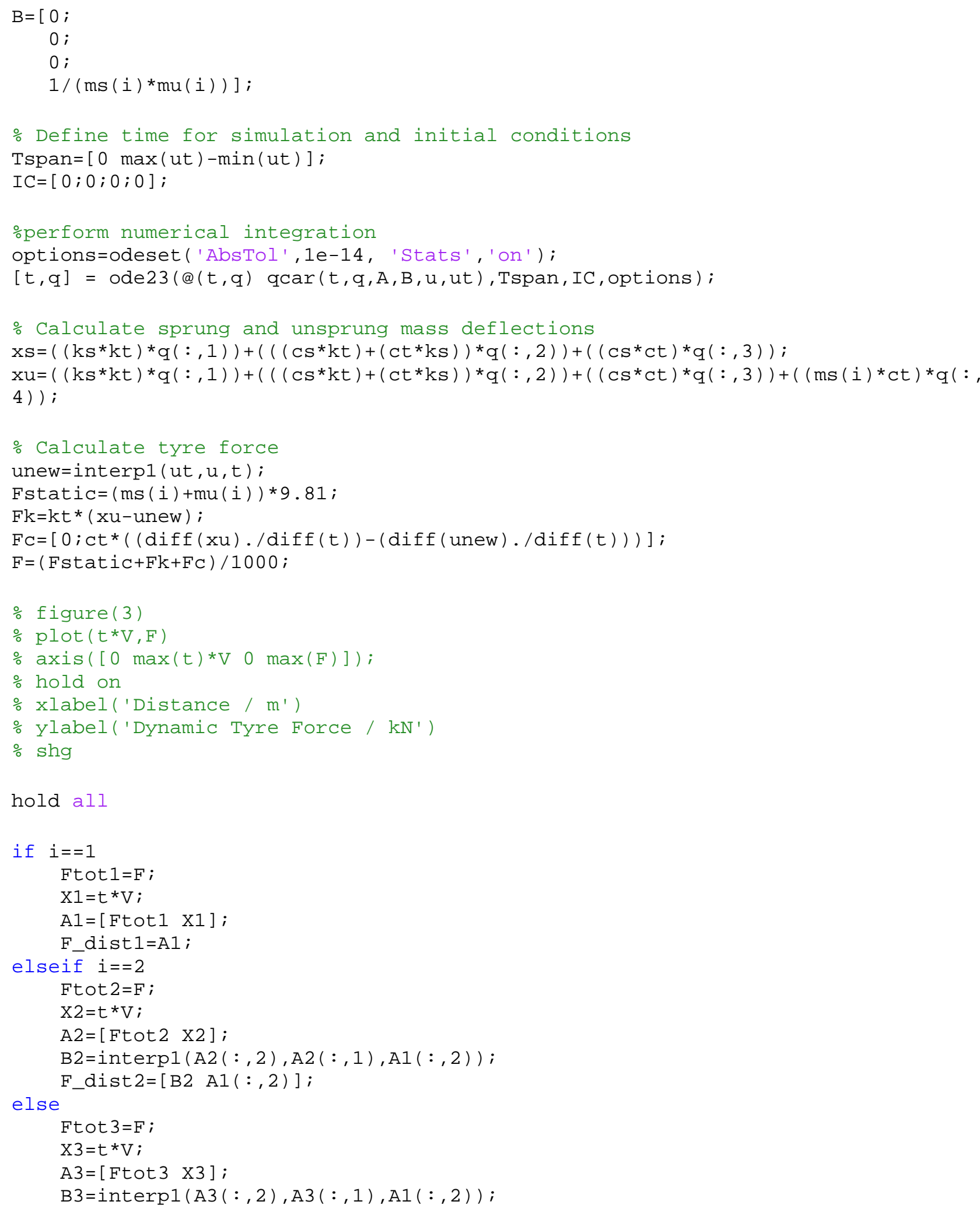


F_dist $3=[B 3 \mathrm{~A} 1(:, 2)]$;

end

Ftotal $=\left[F \_d i s t 1(:, 2)\right.$ F_dist1 $(:, 1) F \_d i s t 2(:, 1)$ F_dist3 $\left.(:, 1)\right]$;

end

Force=interp1(Ftotal $(:, 1)$, Ftotal $(:, 2: 4), j)$;

Force_stat $=$ ones $($ size $($ Force $))$;

for $i=1:$ length (ms);

end

Force_stat $(:, i,:)=(\mathrm{mu}(i)+m s(i)) * 9.81 / 1000$; 